\title{
WestVirginiaUniversity
}

THE RESEARCH REPOSITORY @ WVU

Graduate Theses, Dissertations, and Problem Reports

2005

\section{Stability monitoring and analysis of online learning neural networks}

Sampath Yerramalla

West Virginia University

Follow this and additional works at: https://researchrepository.wvu.edu/etd

\section{Recommended Citation}

Yerramalla, Sampath, "Stability monitoring and analysis of online learning neural networks" (2005). Graduate Theses, Dissertations, and Problem Reports. 2269.

https://researchrepository.wvu.edu/etd/2269

This Dissertation is protected by copyright and/or related rights. It has been brought to you by the The Research Repository @ WVU with permission from the rights-holder(s). You are free to use this Dissertation in any way that is permitted by the copyright and related rights legislation that applies to your use. For other uses you must obtain permission from the rights-holder(s) directly, unless additional rights are indicated by a Creative Commons license in the record and/ or on the work itself. This Dissertation has been accepted for inclusion in WVU Graduate Theses, Dissertations, and Problem Reports collection by an authorized administrator of The Research Repository @ WVU.

For more information, please contact researchrepository@mail.wvu.edu. 


\title{
Stability Monitoring and Analysis of Online Learning Neural Networks
}

\author{
Sampath Yerramalla \\ Dissertation submitted to \\ the College of Engineering and Mineral Resources \\ at West Virginia University \\ in partial fulfillment of the requirements \\ for the degree of \\ Doctor of Philosophy \\ in \\ Electrical Engineering \\ Bojan Cukic, Ph.D., Chair \\ Edgar Fuller, Ph.D. \\ Muhammad A. Choudhry, Ph.D. \\ Katerina D. Goseva-Popstojanova, Ph.D.
}

Arun A. Ross, Ph.D.

Lane Department of Computer Science and Electrical Engineering

Morgantown, West Virginia

2005

Keywords: Neural Networks, Self-Organization Maps, Lyapunov Stability, Validation, and Adaptive Flight Control

Copyright @ 2005 Sampath Yerramalla 


\title{
Abstract \\ Stability Monitoring and Analysis of Online Learning Neural Networks
}

\author{
Sampath Yerramalla
}

On-line adaptation using soft-computational learning methods is on the rise for use in safety-critical applications such as fault-tolerant flight control, maintenance of distributed networks, implementation of high security devices, etc. The inapplicability of traditional analysis methods is limiting the wider use of soft-computational learning methods in safetycritical applications that involve online adaptation. The focus of the research is the development of non-conventional analysis techniques for the testing, verification, validation and analysis of adaptive learning components such as the online learning neural networks.

Our research considers stability of online adaptation as a heuristic measure of correctness in the operation of the adaptive component. The approach is based on the principles of stability according to Lyapunov theory, deriving mathematical stability proofs to assure convergence in neural network learning within a bounded amount of time. The analysis is applied to online learning neural networks such as the Dynamic Cell Structures, Sigma-Pi, and Adaline. This approach is applicable for learning from stationary, non-varying data. For time-varying training data sets, we developed the online stability monitoring methodology. Stability monitors analyze the neural network's learning in real-time. ROC curves present the performance of the developed stability monitoring system as a trade off between the selectivity and sensitivity of the stability detection. Further, we derived a convergence prediction methodology that, given the amplitude of the disturbance, predicts the number of learning cycles required by the neural network to return to a stable state. Our research identifies the significance of topology preservation for a stable online adaptation. In order to improve the robustness of topology preservation, we propose a modified DCS learning algorithm.

Our dissertation offers the first known methodology for verification, validation and analysis of learning in adaptive computing applications. The developed techniques can overcome the difficulties associated with model-uncertainty in the context of assurance of adaptive systems. 
"To my family" 


\section{Acknowledgments}

“...Many hands, hearts, and minds generally contribute to anyone's notable

achievements".

-Walt Disney

The dissertation could not have been successfully completed without the guidance of my academic advisors, Dr. Bojan Cukic and Dr. Edgar Fuller. I wish to thank Dr. Cukic for providing me gracious financial support and teaching me to accept nothing less than my best efforts. Dr. Fuller's thought-provoking advices have contributed immensely to shaping my dissertation. I would also like to thank the other advisory committee members, Muhammad A. Choudhry, Dr. Katerina D. Goseva-Popstojanova, and Dr. Arun A. Ross for their comments and reviews. I am grateful for the support that I received from the members of the Institute for Scientific Research, Inc. especially from Margie, Brian, and Spiro. I extend my thanks to friends and colleagues for their support and help.

I am grateful to thank my wife, Shailu, for her patience and endurance throughout the process. Finally, I wish to thank the members of my family for their encouragement. 


\section{Contents}

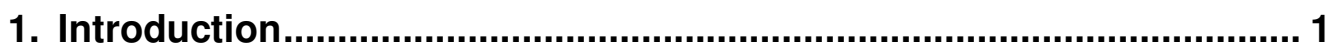

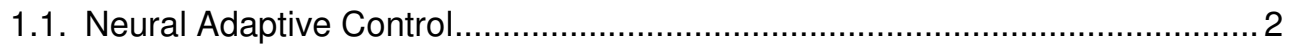

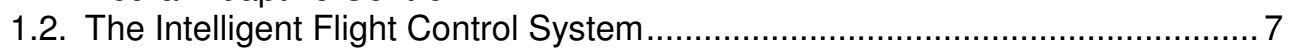

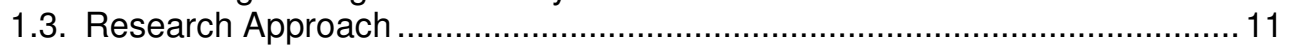

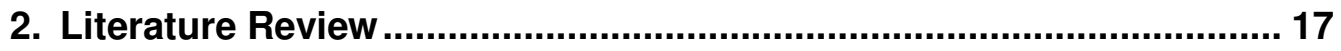

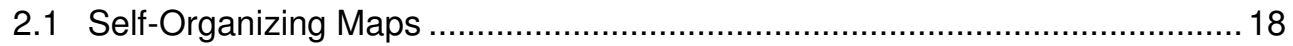

2.1.1 Training Process in Self-Organizing Maps .............................................. 18

2.1.2 Convergence Properties of Self-Organizing Maps ……................................22

2.2 Dynamic Cell Structures Neural Networks ……............................................26

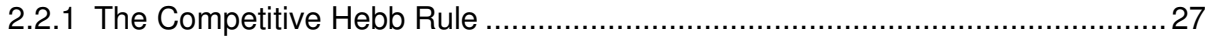

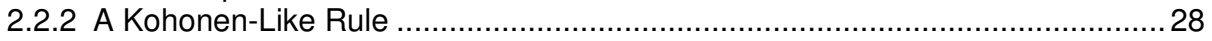

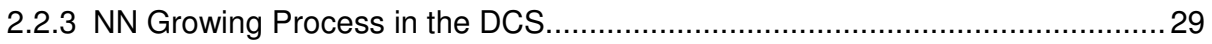

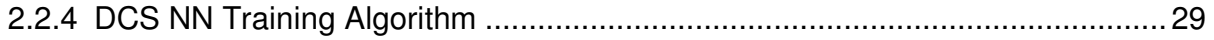

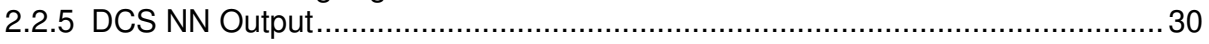

2.3 Limitations of Traditional NN Analysis Methods ........................................... 32

2.3.1 Formal Methods .................................................................................. 32

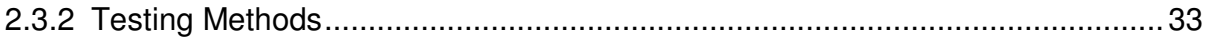

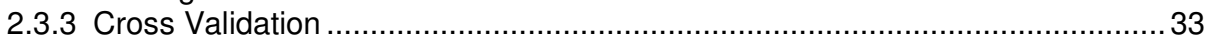

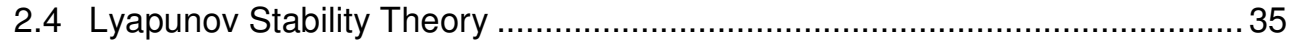

3. Theoretical Stability Analysis .............................................................. 40

3.1. Learning Dynamics of the DCS .............................................................. 41

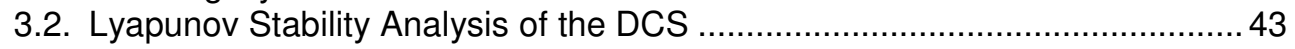

4. Online Stability Monitoring ............................................................... 53

4.1. Mathematical Formulation of Stability Monitors ..........................................55

4.2. Multiple-Monitor Based Approach - Why? .............................................5 57

4.3. Neural Network Convergence Prediction .................................................... 76

4.3.1. Convergence Prediction of the BMU Errors ................................................. 80

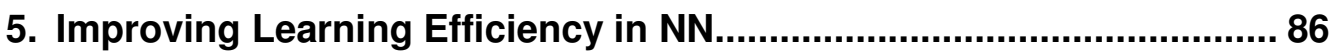

5.1. Conditions Leading to Loss of Topology in SOMs..................................... 91

5.2. An Improvised Neural Network Growing Process ......................................... 97

6. Generalization of NN Analysis ....................................................... 121

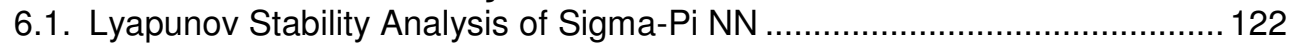

6.2. Lyapunov Stability Analysis of ADALINE NN ........................................ 144

7. Conclusions and Future Work ………............................................. 156

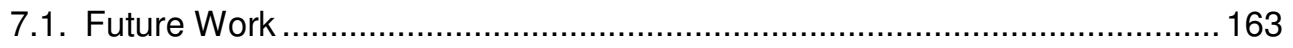




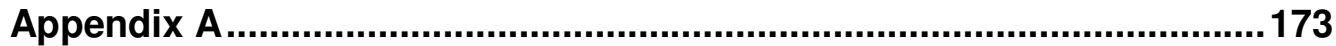

Proof for Theorem 3.1 Stability of the DCS ............................................... 173

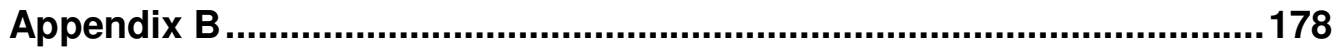

Proof For Theorem 4.1: Convergence of the BMU Error ..................................178

Proof For Theorem 4.2: Convergence of the Q-BMU Error ................................ 179

Appendix C .

Proof for Theorem 5.1: Disconnected Highest Resource Node ........................... 180

Proof for Theorem 5.2: Loss of Topology Preserving Properties ......................... 182

Proof for Theorem 5.3: Efficient Topology Preservation ..................................... 186 


\section{List of Figures}

Figure 1.1 Illustration of the concept of neural network approximation ................................... 3

Figure 1.2 Architectural overview of a NN based indirect adaptive controller ........................... 4

Figure 1.3 Architectural overview of a NN based direct adaptive controller ............................. 6

Figure 1.4 Control regions in a NN based direct adaptive controller ...................................... 7

Figure 1.5 Control architecture of the First Generation IFCS Implementation ......................... 9

Figure 1.6 Test bed aircraft for evaluation of IFCS implementation ...................................... 11

Figure 1.7 Framework of the proposed non-conventional analysis approach......................... 15

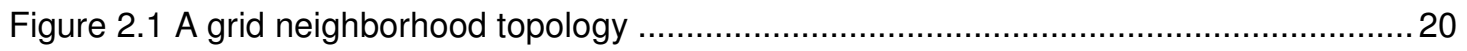

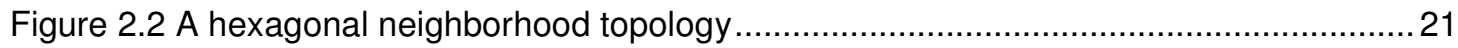

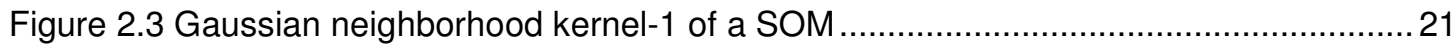

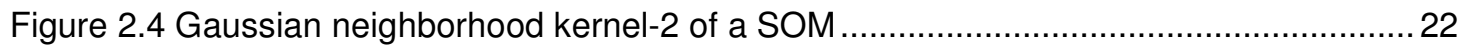

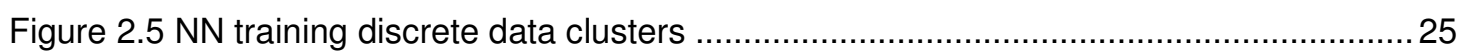

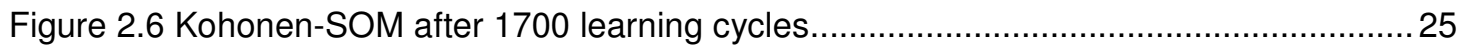

Figure 2.7 Control Flow Diagram of the Online Learning DCS Neural Network ....................... 31

Figure 2.8 Illustration of local stability concept of a dynamic system ...................................... 37

Figure 3.1 Snapshot of the DCS NN initiation with two connected nodes................................ 47

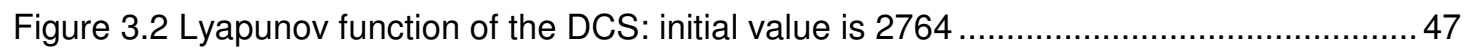

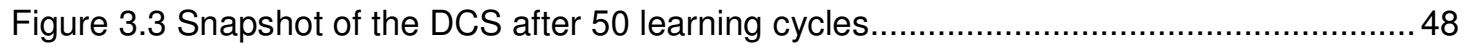

Figure 3.4 Lyapunov function of the DCS after 50 learning cycles ...................................... 48

Figure 3.5 Snapshot of the DCS after 100 learning cycles.................................................. 49

Figure 3.6 Lyapunov function of the DCS after 100 learning cycles ....................................... 49

Figure 3.7 Snapshot of the DCS after 150 learning cycles................................................. 50

Figure 3.8 Lyapunov function of the DCS after 150 learning cycles ....................................50

Figure 3.9 Snapshot of the DCS after 200 learning cycles............................................... 51

Figure 3.10 Laypunov function of the DCS after 200 learning cycles ...................................51

Figure 4.1 User interface of NASA-WVU adaptive flight simulator........................................5 58

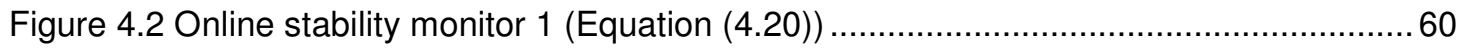

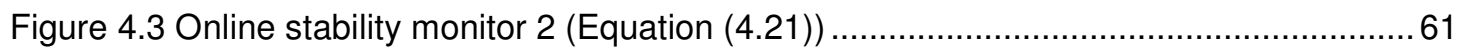

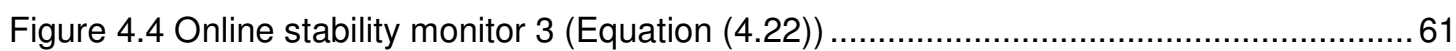

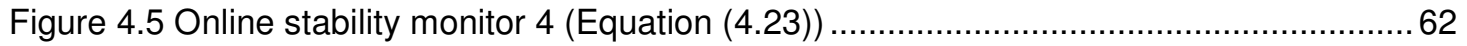

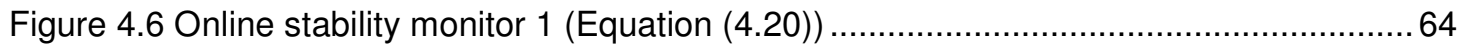

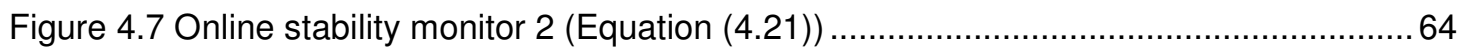

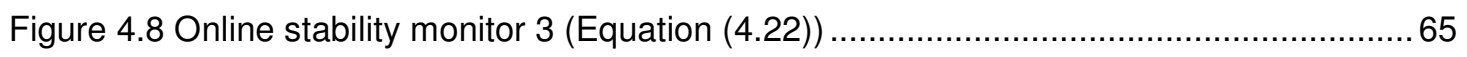

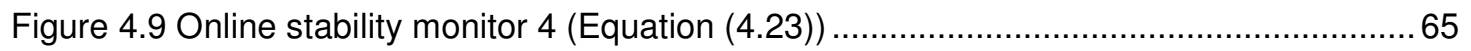




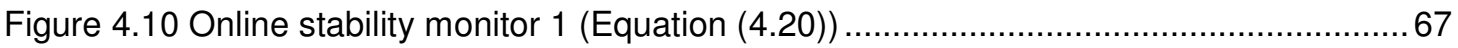

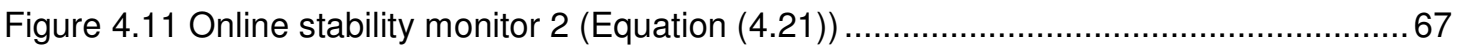

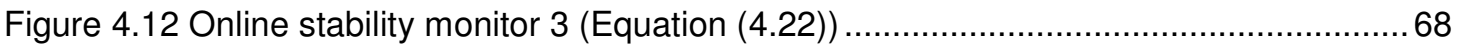

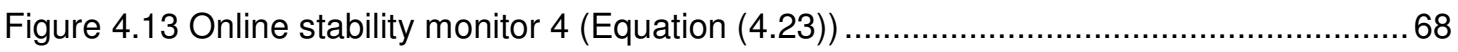

Figure 4.14 ROC curve of fused stability monitors for failure mode 1 (Table 4-1) ................... 72

Figure 4.15 ROC curve of fused stability monitors for failure mode 2 (Table 4-1) ................... 72

Figure 4.16 ROC curve of fused stability monitors for failure mode 3 (Table 4-1) .................. 73

Figure 4.17 ROC curve of fused stability monitors for failure mode 4 (Table 4-1) ................... 73

Figure $4.18 \mathrm{ROC}$ curve of fused stability monitors for failure mode 5 (Table 4-1) ................... 74

Figure 4.19 ROC curve of fused stability monitors for failure mode 6 (Table 4-1) .................. 75

Figure 4.20 ROC curve of fused stability monitors for failure mode 7 (Table 4-1) .................. 75

Figure 4.21 Convergence of Monitor 1 back to stability in 33 learning cycles........................... 78

Figure 4.22 Convergence of Monitor 2 back to stability in 34 learning cycles.......................... 78

Figure 4.23 Convergence of Monitor 3 back to stability in 28 learning cycles........................... 79

Figure 4.24 Monitor 4 shows no response to the single point data disturbance ...................... 79

Figure 4.25 Results from predicting convergence in the BMU error ....................................... 84

Figure 4.26 Results from predicting convergence in the quantized BMU error........................ 84

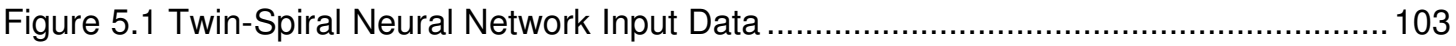

Figure 5.2 DCS using the original NN growing algorithm after 114 cycles........................... 104

Figure 5.3 DCS using the modified NN growing algorithm after 114 cycles.......................... 104

Figure 5.4 DCS using the original NN growing algorithm after 115 cycles............................ 106

Figure 5.5 DCS using the modified NN growing algorithm after 115 cycles......................... 106

Figure 5.6 DCS using the original NN growing algorithm after 120 cycles........................... 107

Figure 5.7 DCS using the modified NN growing algorithm after 120 cycles......................... 107

Figure 5.8 DCS using the original NN growing algorithm after 128 cycles............................. 108

Figure 5.9 DCS using the modified NN growing algorithm after 128 cycles......................... 108

Figure 5.10 DCS using the original NN growing algorithm after 131 cycles.........................110

Figure 5.11 DCS using the modified NN growing algorithm after 131 cycles....................... 110

Figure 5.12 DCS using the original NN growing algorithm after 143 cycles..........................111

Figure 5.13 DCS using the modified NN growing algorithm after 143 cycles....................... 112

Figure 5.14 DCS using the original NN growing algorithm after 152 cycles......................... 113

Figure 5.15 DCS using the modified NN growing algorithm after 152 cycles........................ 113

Figure 5.16 DCS using the original NN growing algorithm after 172 cycles.......................... 114

Figure 5.17 DCS using the modified NN growing algorithm after 172 cycles........................ 115

Figure 5.18 DCS using the original NN growing algorithm after 180 cycles..........................116

Figure 5.19 DCS using the modified NN growing algorithm after 180 cycles........................116 
Figure 5.20 DCS using the original NN growing algorithm after 305 cycles. 118

Figure 5.21 DCS using the modified NN growing algorithm after 305 cycles 118

Figure 6.1 Control architecture of the Generation II of the IFCS ...................................... 122

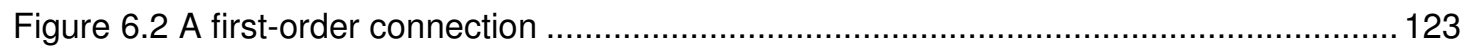

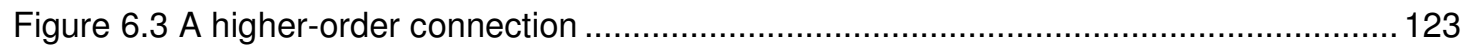

Figure 6.4 A Fully Connected Higher Order Neural Network ............................................. 125

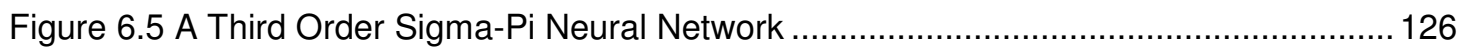

Figure 6.6 E-modified Neural Network Learning Algorithm ............................................... 127

Figure 6.7 Matlab Simulink implementation of online learning S-Pi NN ............................... 129

Figure 6.8 Matlab Simulink subsystem of the Roll S-Pi NN block of Figure 6.7 ................... 129

Figure 6.9 Matlab Simulink subsystem of the Weight Update block of Figure 6.8 ............... 130

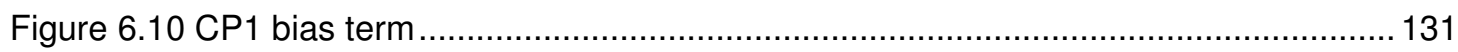

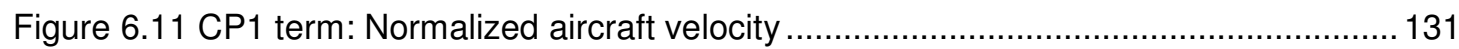

Figure 6.12 CP1 term: Square of normalized aircraft velocity ............................................ 132

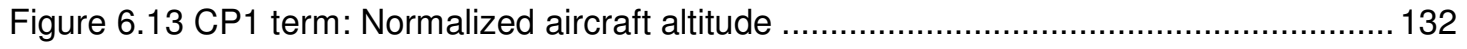

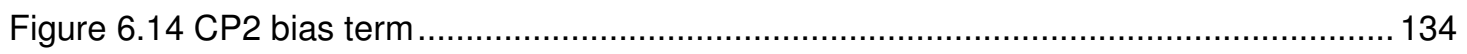

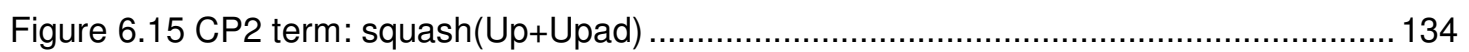

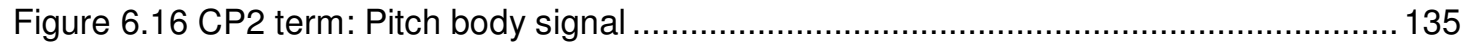

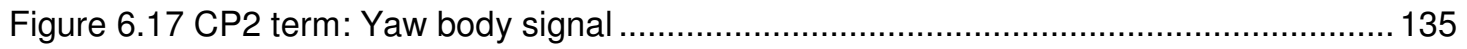

Figure 6.18 CP2 term: Reference model roll acceleration................................................. 136

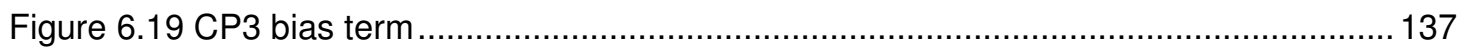

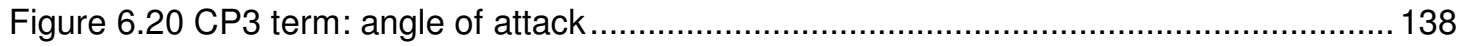

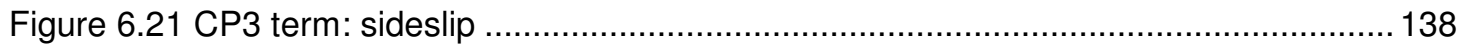

Figure $6.2260 \mathrm{BP}$ terms for the roll-channel S-Pi ......................................................... 139

Figure 6.2360 Acceleration error terms for the roll-channel S-Pi ........................................ 139

Figure 6.24 Adaptation of the 60 roll-channel S-Pi weight terms ...................................... 140

Figure 6.25 Online monitor 1: zero order weights adaptation monitor ................................ 141

Figure 6.26 Online monitor 2: first order weights adaptation monitor................................... 142

Figure 6.27 Online monitor 3 : second order weights adaptation monitor............................... 142

Figure 6.28 Output from the current implementation of roll channel S-Pi NN ..................... 143

Figure 6.29 Output from the IFCS implementation of roll channel S-Pi NN ......................... 144

Figure 6.30 A single ADALINE neural network with two inputs .......................................... 145

Figure $6.316 \mathrm{BP}$ terms for the roll-channel ADALINE ........................................................ 149

Figure 6.326 Acceleration error terms for the roll-channel ADALINE................................... 149

Figure 6.336 Roll channel acceleration error terms for times 12 to 14 seconds ................... 150

Figure 6.34 Adaptation of the 6 roll-channel ADALINE weight terms ................................. 150 


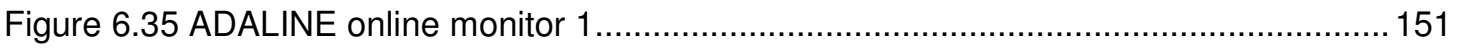

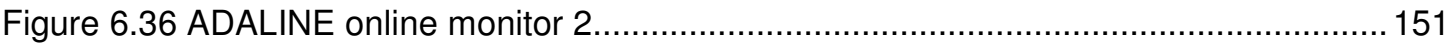

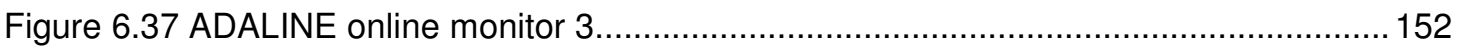

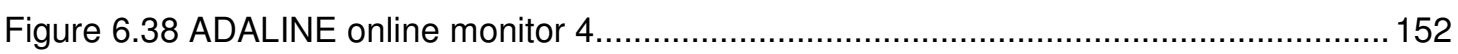

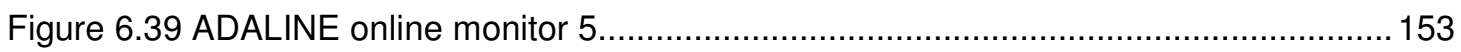

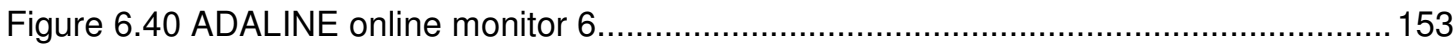

Figure 6.41 Output from the current implementation of roll ADALINE NN ........................... 154

Figure 6.42 Output from the IFCS implementation of roll ADALINE NN .............................. 154 


\section{List of Tables}

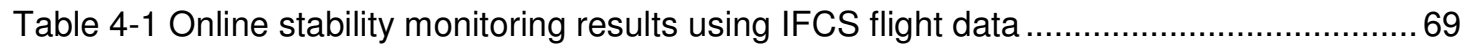

Table 5-1 Comparison of original and modified NN growing algorithms ...... 119 


\section{Table of Definitions}

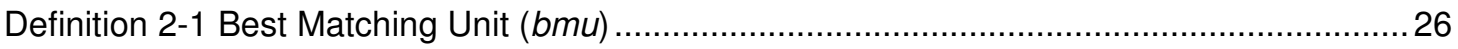

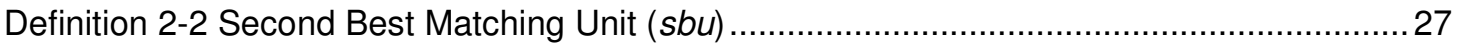

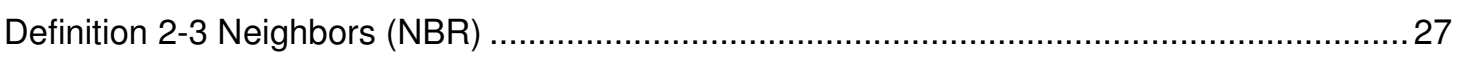

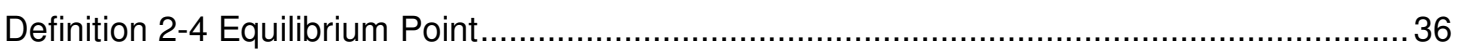

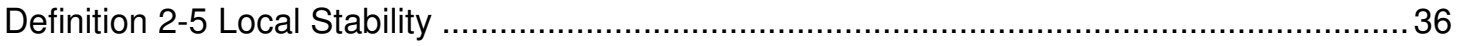

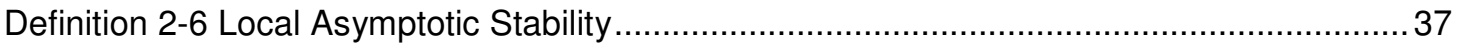

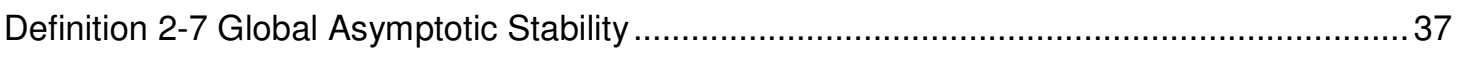

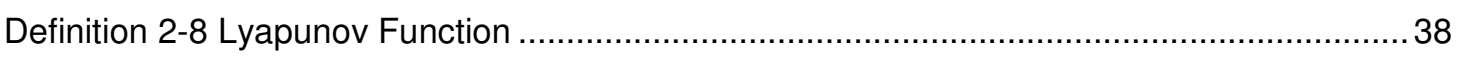

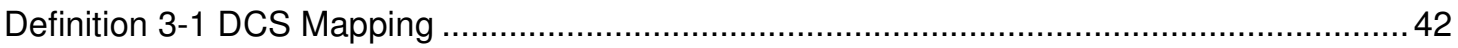

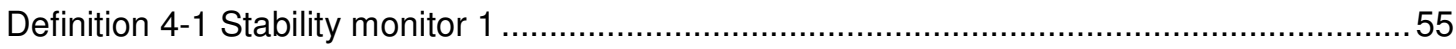

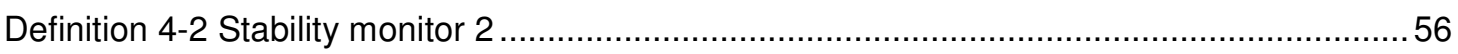

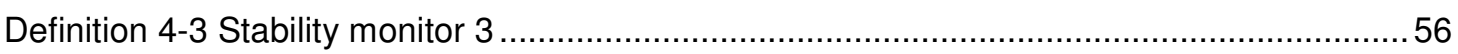

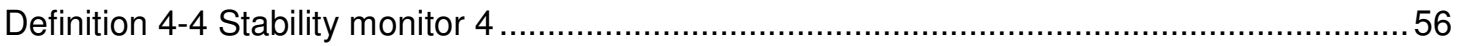




\section{Table of Theorems}

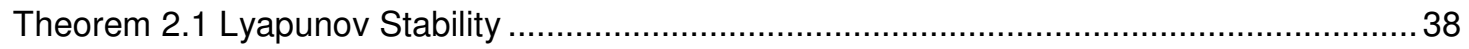

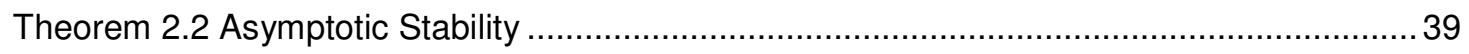

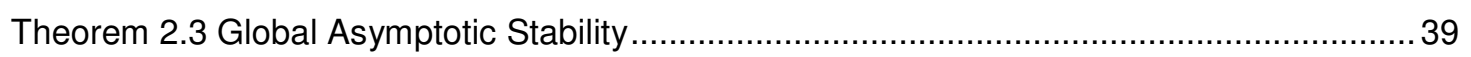

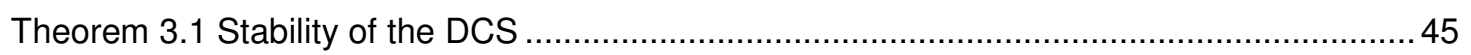

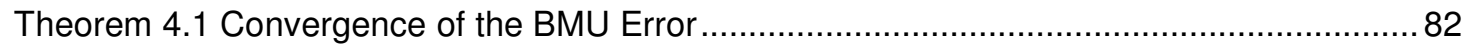

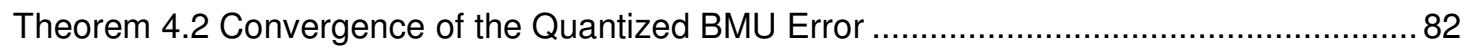

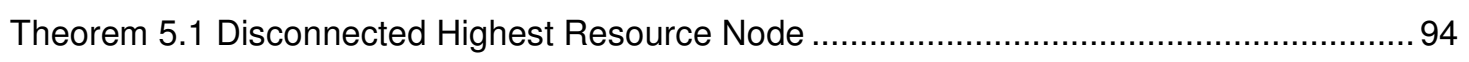

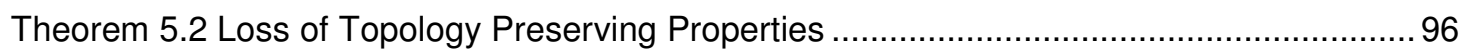

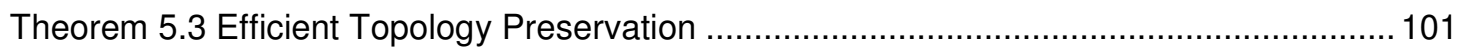




\section{Ch a p ter}

\section{Introduction}

The model-dependant nature of the operation of traditional systems makes them a desirable choice for standard applications where cost-effectiveness is a priority - data mining, marketing and finance evaluations, etc. Adaptive systems on the other hand have the ability to deal with system-uncertainty and are therefore suited for applications where safety and reliability are the utmost of the concerns - fault tolerant aircraft control, automated navigation, control of missiles, sensor failure detection, maintenance of distributed networks, implementing high security devices, and medical applications [40, 42, 48, 53, 90, 91, 96].

An adaptive control system can be defined as a system that has a means to sense its environment, process the sensed information in a timely manner, and optimize its performance by adjusting its behavior using closed-loop control actions. If the learning and adaptation occur after the system deployment, then it is called online adaptation. It is desired to have an adaptive control system capable of coping with unforeseen changes in the environment or failures within the system through online adaptation $[47,55,85,87,96,97]$.

Adaptive control is a preferred approach for providing stability in nonlinear systems with uncertain dynamics. The goal of adaptive control is to guarantee the stability and performance of the system in the midst of un-modeled dynamics and external disturbance. Taylor et al. and Sastry et al. originally proposed adaptive control of linear systems [46, 47, 53]. A systematic approach for designing adaptive controllers for linear systems was presented by Kanellakopoulos et al. [41, 47]. The first of its kind, the model reference adaptive control (MRAC) was developed by Whitaker et al. at the M.I.T. Instrumentation Laboratory in $1958[51]$. 
Using neural networks for online adaptation in adaptive control is a relatively novel approach for creating a fault-tolerant control system that is capable of recuperating from system failures $[55,89,92,94,96,97]$. Therefore, there is a growing interest in experimenting, developing and implementing nonlinear adaptive control systems using neural network approximation methods $[83,85,86,88,90]$. Section 1.1 of this chapter describes the role of neural networks in adaptive control.

Adaptive controller techniques can be classified into two major types: the indirect and the direct adaptive control. In feedback linearized indirect adaptive control, the system parameters are first estimated (using a parameter estimation algorithm or a neural network) and then used in calculating the control parameters (using a controller or a neural network) $[40,44,68]$. In direct adaptive control, the control parameters are adjusted directly without any prior estimation $[83,85,86]$. Section 1.1 of this chapter describes control concepts relative to the feedback linearized indirect adaptive control. Section 1.1 also describes control concepts relative to the neural network based direct adaptive control. The control architecture of the Intelligent Flight Control System (IFCS), a practical implementation of the neural network based adaptive control, is described in the Section 1.2. Finally, Section 1.3 describes some difficulties relative to the analysis of online adaptive neural networks that are embedded in adaptive systems. In order to overcome these difficulties in validating adaptive neural networks for safety-critical applications such as the aircraft control in the IFCS, Section 1.3 also presents a detailed research proposal for developing non-conventional analysis methods suitable for evaluation and testing of online adaptive neural networks.

\subsection{Neural Adaptive Control}

Neural networks have been researched for the past fifty years as biologically inspired soft-computing learning paradigms composed of a large number of computational units called neurons. The abilities of neural networks for approximating unknown functions to desired levels of accuracy are well known $[23,25,29,38]$. Neural networks are usually preferred for 
solving optimization problems that are otherwise hard to realize using a conventional, algorithmic approach i.e., following a set of rules in order to solve a problem.

The role of a neural network approximation in a control application is usually to produce similar responses to those of the unknown function, when both the neural network, and the unknown function are given the same inputs (test inputs). This concept is further illustrated in Figure 1.1

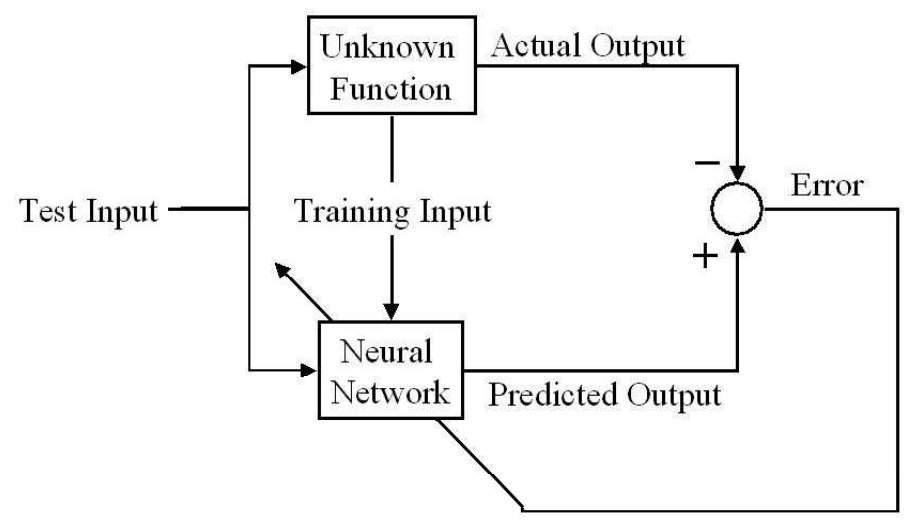

Figure 1.1 Illustration of the concept of neural network approximation

If the unknown function of Figure 1.1 corresponds to the values of parameters of the system being controlled, then the neural network is considered as a parameter identification model, the PID [68]. If the unknown function corresponds to the inverse of the system being controlled, then the neural network behaves as the controller [68, 85]. Adaptive control using neural networks is especially useful in situations where it is almost impossible to estimate beforehand uncertainties and un-modeled system dynamics - for example, fault tolerant control of an aircraft following a major system failure or a combat damage $[40,42,43,49,50$, $83,84,85,86,91]$.

The term "indirect" in indirect adaptive control refers to the control where the system parameters are first estimated and then used for calculating the control parameters. One can use neural networks for both parameter estimation and control. Feedback linearization is an 
example of indirect adaptive control approach. In feedback linearization technique, the dynamics of a nonlinear system are transformed into a linear system using feedback from the system. Indirect adaptive control using neural networks for online adaptation in feedbacklinearized systems has proven to be an effective means of designing and implementing faulttolerant controllers $[40,44,68]$. The control architecture of an indirect adaptive controller using feedback linearization is shown in Figure 1.2. The control signal from the adaptive feedback linearization controller is composed of two essential parts, the first part is used in canceling the nonlinearities, and the second part is used for the linear state feedback.

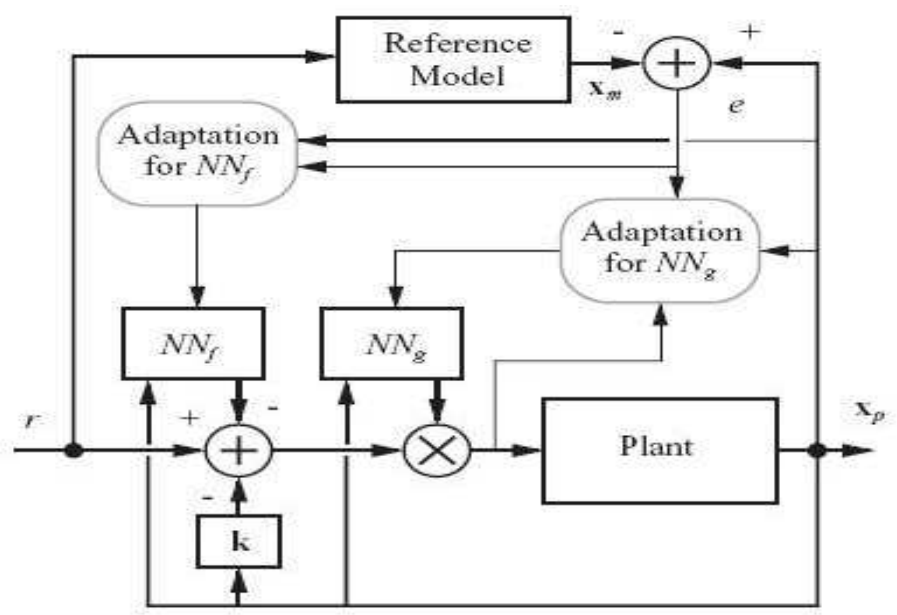

Figure 1.2 Architectural overview of a NN based indirect adaptive controller

The types of system for which this control technique is applicable are generally of the form

$$
\begin{aligned}
& x_{p}{ }^{\mathrm{n}}=f\left(\mathbf{x}_{p}\right)+g\left(\mathbf{x}_{p}\right) u \\
& u=g^{-1}\left(\mathbf{x}_{p}\right)\left[-f\left(\mathbf{x}_{p}\right)-\mathbf{k}^{\mathrm{T}} \mathbf{x}_{p}+r\right]
\end{aligned}
$$


In Equation (1.1), $x_{p}{ }^{n}$ represents the $n^{\text {th }}$ derivative of the state of the plant (system being controlled), $\mathbf{x}_{p}=\left[x_{p} \dot{x}_{p} \ddot{x}_{p} \mathrm{~K} x_{p}{ }^{\mathrm{n}-1}\right]^{\mathrm{T}}$ represents the previously recorded states of the system being controlled, $u$ represents the control signal that is required to control the system, $r$ represents the reference input, and $k$ is the feedback control gain. The nonlinear system shown in (1.1) can be transformed into a linear form as shown below.

$$
x_{p}{ }^{\mathrm{n}}=-\mathbf{k}^{\mathrm{T}} \mathbf{x}_{p}+r
$$

As shown in Figure 1.2, using neural networks, one can approximate the nonlinear functions $f$ and $g^{-1}$ in (1.1). Let us consider two neural networks $N N_{f}$ and $N N_{g}$ that can approximate the nonlinear functions $f$ and $g^{-1}$ in (1.1) respectively. The control signal of (1.1) can be then be rewritten in the following manner.

$$
u=N N_{\mathrm{g}}\left[-N N_{\mathrm{f}}-\mathbf{k}^{\mathrm{T}} \mathbf{x}_{p}+r\right]
$$

It can therefore be said that the neural networks, $N N_{f}$ and $N N_{g}$ in (1.3) represent the plant identification model and the controller respectively. The control error, e generated in following the reference model is given by the following equation.

$$
\begin{aligned}
& x_{m}{ }^{\mathrm{n}}=-\mathbf{k}^{\mathrm{T}} \mathbf{x}_{m}+r \\
& e=\mathbf{x}_{p}-\mathbf{x}_{m}
\end{aligned}
$$

In the Equation (1.4), $x_{m}{ }^{n}$ represents the state of the system as modeled by the NN (from Equation (1.3)). With the use of a good approximating neural network learning algorithm, it can be shown that the error, $e$ in the Equation (1.4) converges to zero [68].

The term "direct" in direct adaptive control implies that the control parameters are adjusted directly without prior estimation of the control parameters for example, using an augmented control signal. Figure 1.3 shows the architectural overview of a NN based direct 
adaptive controller. A direct adaptive control essentially consists of three controller components.

- $\quad$ Linear feedback controller, $u_{p d}(t)$

- $\quad$ Sliding mode controller, $u_{s l}(t)$

- A neural adaptive controller, $u_{a d}(t)$

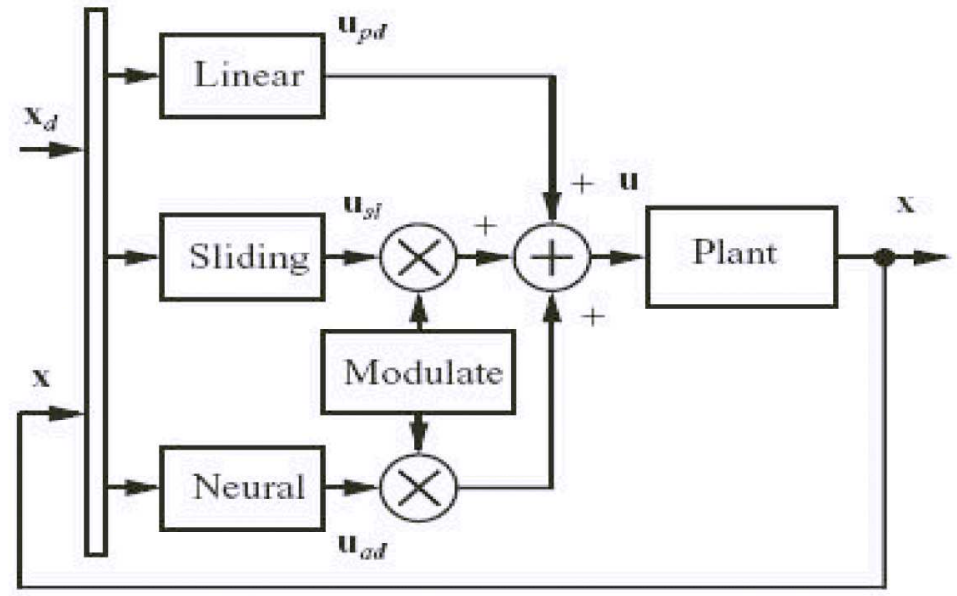

Figure 1.3 Architectural overview of a NN based direct adaptive controller

The total control signal from the control system is given in the Equation (1.5). The term, $m(t)$ allows for smooth transition between the sliding mode and the adaptive controllers. Based on the location of the state of the system, the function $m(t)$ can be chosen appropriately.

$$
u(t)=u_{p d}(t)+(1-m(t)) u_{a d}(t)+m(t) u_{s l}(t)
$$

Figure 1.4 shows the control regions in a neural network based direct adaptive controller [68]. The condition for determining the control regions is as follows. 


$$
\begin{array}{lrr}
m(t)=0 \quad \text { if } & x(t) \in A_{d} \\
m(t)=1 \quad \text { if } & x(t) \in A_{c} \\
0<m(t)<1 & \text { otherwise }
\end{array}
$$

As shown in Figure 1.4 the sliding mode controller is used to keep the system state in a region where the neural network can be accurately trained to achieve optimal control [68]. When the system gets out of this region, the sliding mode controller is switched on and the neural controller is turned off. The combination of these controllers generates a control signal that is capable of adapting for an optimized performance.

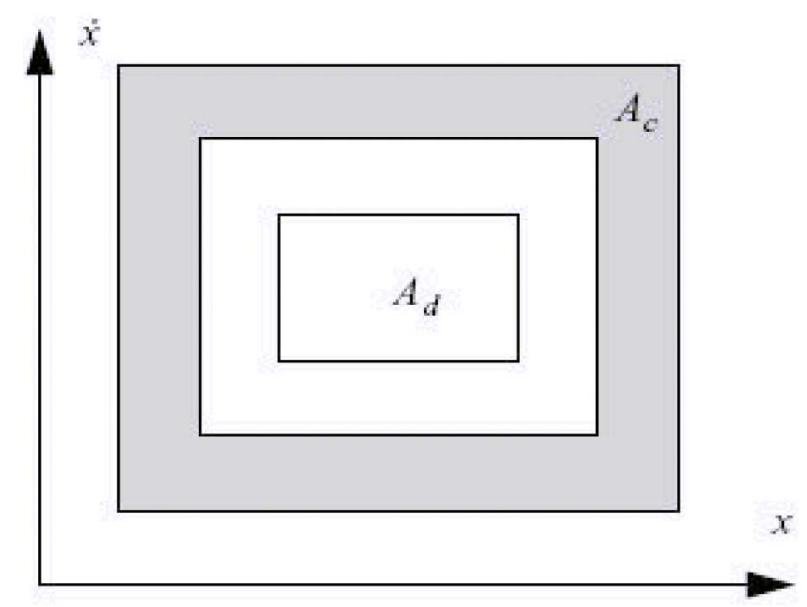

Figure 1.4 Control regions in a NN based direct adaptive controller

\subsection{The Intelligent Flight Control System}

Design of flight controllers has been dominated by the classical control techniques for several years. While the classical control techniques deliver effective flight controllers, the control implementations are severely restricted in operation to nominal or no-failure flight conditions. In the last 30 years, at least 10 aircrafts have experienced major flight failures claiming more than 1100 lives [48]. Some of these failures have been for example, stuck or broken ailerons, rudders, and stabilators, and/or faulty sensors. Therefore, there is a growing 
interest in developing adaptive controllers for implementing fault-tolerant flight control capabilities following system failures [40, 42, 43, 49, 50, 83, 84, 85, 86, 91].

The use of biologically inspired soft computational learning techniques (neural networks, fuzzy logic, and Al planners) for online adaptation has revolutionized the operation of real-time automation and control. Practical implementations of adaptive controllers in military aircrafts (F/B-15, F/A-18, AH-64, XV-15, tailless $\mathrm{X}-36$, and other fighter aircrafts) for enhanced stability and performance demonstrates a superior fault-tolerant flight control capability $[40,42,43,49,50,83,84,85,86,91]$. Experimental success suggests significant potential for developing and deploying fault-tolerant flight controllers for futuristic civilian airplanes [49].

Implementing a fault-tolerant flight control system is a challenging task as constructing it with guaranteed stability that ensures peak performance of the aircraft requires a thorough understanding of the objective functions. In the recent years, NASA has conducted series of experiments evaluating soft-computational learning techniques (neural networks, Al planners) for providing fault-tolerant control capabilities in flight control systems following sensor and/or actuator failures $[40,48,49,50,85]$. A prototype of this is NASA's, Intelligent Flight Control System (IFCS). Figure 1.5 depicts an architectural overview of the NASA's first generation of the IFCS implementation using Online Learning Neural Network (OLNN). The IFCS system was primarily designed to incorporate online learning neural network concepts into flight control software evaluated for optimized aircraft performance during nominal and multiple accident and/or an off-nominal flight scenarios.

The four main components of the first generation of the IFCS implementation that are shown in Figure 1.5 are as follows.

- A baseline or pre-trained neural network (PTNN)

- A parameter identification/estimation (PID) algorithm

- An online learning neural network, in this case the Dynamic Cell Structures (DCS) 
- A control algorithm: the Stochastic Optimal Feedforward Feedback Technique (SOFFT)

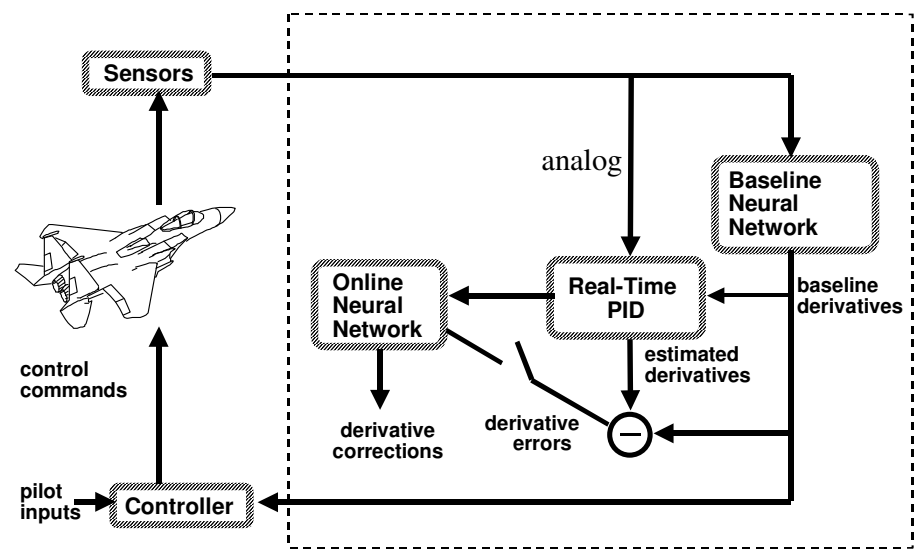

Figure 1.5 Control architecture of the First Generation IFCS Implementation

A baseline or Pre-Trained Neural Network (PTNN) is used for replacing linear maps from standard designs (like the reference adaptive control) in order to produce a robust system that can handle nonlinearities. The PTNN is a neural network algorithm that is trained to produce stability and control derivatives depending on a specific flight condition. It is essentially a table-lookup scheme based on wind-tunnel experiments for the stable aircraft conditions. It learns the stability and control derivatives from the wind tunnel data before flight and is fixed throughout the flight. The PTNN provides the first best estimate of the aircraft's dynamic characteristics $[40,49,50]$. PTNN is non-adaptive, meaning that once trained, it does not change during the flight. The PTNN is comprised of 34 distinct Multi-Layer Perceptron (MLP) neural networks with some of the networks' output combined to form the derivatives. The neural networks are trained with two different training techniques: a modification of Active Selection, and the Levenberg-Marquardt algorithm [40, 49, 50].

The adaptive nature of the IFCS system is due to the real-time Parameter Identification (PID) operation. It essentially uses an equation-error technique employing a 
Fourier transform regression algorithm. The PID algorithm is an online function that determines the actual stability and control characteristics of the aircraft during the flight. In the case of a catastrophic failure in the flight-conditions (stuck stabilators, broken ailerons, etc,), notable discrepancies between the outputs of the PTNN and the PID are generated in realtime. The difference in the values of the PTNN and the PID is called stability and control derivative (SCD) error. SCD error indicates conditions that fall outside the scope of traditional (linearized) control gain look-up tables.

The role of OLNN (see Figure 1.5) is to learn and represent the SCD errors in realtime, and provide a good estimate of the observed SCD errors for system control under new flight conditions. In the first generation of the IFCS implementation, the Dynamic Cell Structure (DCS) neural networks provide the online learning for the system. They track the differences between the PTNN and the PID and provide an organized map of updates to the stability and control derivatives of the aircraft. By accommodating the changing dynamics of the aircraft, the DCS aids the IFCS controller in providing optimized control needed to make the aircraft stable and consequently, safe even under off-nominal flight-conditions. The SOFFT control algorithm uses stability and control derivatives information to fine-tune the system to provide optimal stability and specific flying characteristics $[40,49,50]$.

The test bed aircraft selected to evaluate the IFCS neural network and the flight control system is a highly modified McDonnell-Douglas NF-15B Eagle $[40,49,50]$ that is shown in Figure 1.6. The modified control surfaces are also shown in Figure 1.6. Some of the modifications on this aircraft include additional control surfaces (canards), thrust vectoring nozzles, and a digital fly-by-wire flight control system. The use of canard surfaces along with thrust vectoring nozzles allows for increased maneuverability and stability of the aircraft [49, 50].

The result from the IFCS project will be utilized in an overall strategy aimed at advancing neural network flight control technology to new aerospace systems designs, 
including civil and military aircraft, reusable launch vehicles, uninhabited vehicles, and space vehicles $[49,50]$. The objectives of the IFCS program are summarized below.

- Implement and fly neural network software for flight controls.

- Demonstrate a safe in-flight simulated failure recovery using the neural network learning algorithms and the adaptive flight controller techniques.

- Develop analysis procedures for flight critical checkout of the nondeterministic neural network learning algorithms.

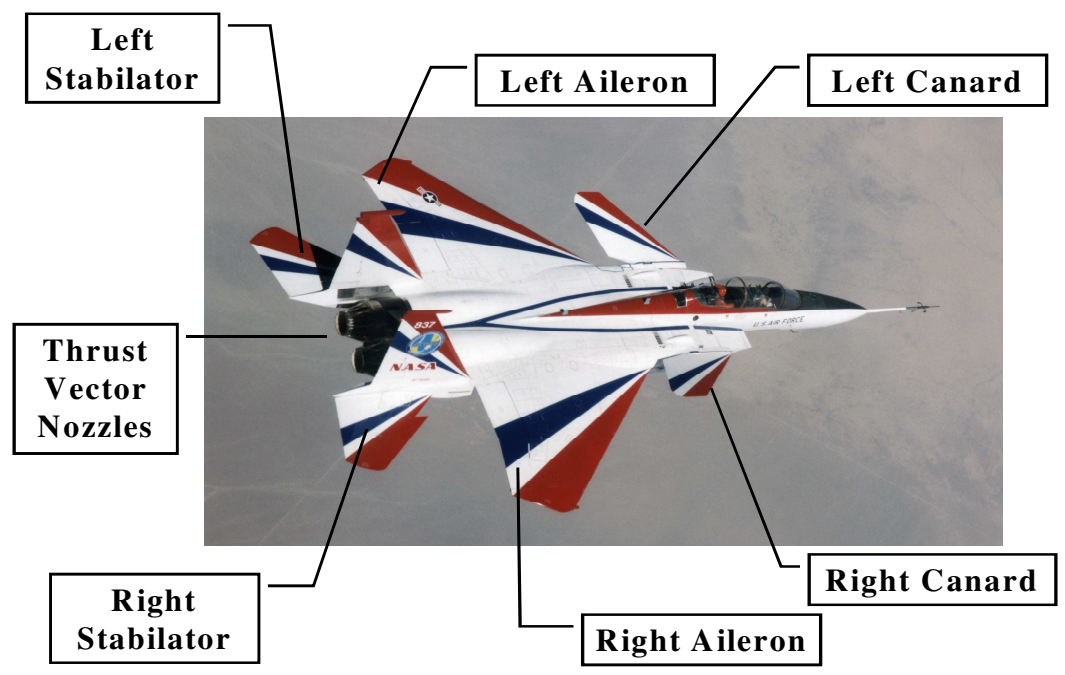

Figure 1.6 Test bed aircraft for evaluation of IFCS implementation

The primary focus of this research is the last of the listed goals of the IFCS program, i.e. to develop a theoretically sound and practically feasible framework for analysis and evaluation of the learning behavior in the nondeterministic online learning neural networks in order to ensure their correct operation.

\subsection{Research Approach}

From the earlier discussions of this chapter, it is evident that the performance of the adaptive control systems is significantly enhanced by the use of adaptive components such as 
the online learning neural network algorithms. It must be realized that by accommodating for changing dynamics of the system, online adaptation plays a critical role in aiding the adaptive control system to recuperate from catastrophic failure conditions. In a flight control system, these failures can be slow and catastrophic (sensor/actuator failures, changed aircraft dynamics: broken aileron or stabilator, etc) $[42,49,83,88,91]$. Therefore, there is a growing need for providing safe and reliable platform for online adaptation in safety-critical applications $[1,5,6,10,12,13,14,69,73,77,78,81]$. As there is no such thing as "perfect adaptation", analysis research is the key for testing and analysis of adaptive components. Adaptation mechanisms (online learning neural networks) in safety-critical systems must therefore be tested and analyzed using practically possible analysis techniques before their deployment into the actual system.

When the neural networks perform online adaptation, its behavior may have a direct consequence on the overlaid adaptive system. It is, therefore, necessary to ensure a correct and safe neural network behavior before its deployment into the safety-critical system $[1,5,6$, $10,12,13,14,69,73,77,78,81]$ The question then is:

- How to derive an analysis procedure for ensuring correct neural network behavior during its online adaptation in safety-critical systems?

Even after decades of extensive research on neural networks and their widespread applications in many real-world systems, the answer to this question remains uncertain and contradictory. Unlike conventional learning methods, the learning process in most neural networks is dependant on the nature of the inputs and evolves non-deterministically over time in an unpredictable manner. In many cases of neural network learning, the inputs to the neural network and outputs from the neural network can be determined, but it is not clear how (and when) the neural network has achieved a certain desired state in learning. Due to this nondeterministic nature of adaptation, neural networks are considered "black box" learning mechanisms $[1,5,6,10,12,13,14]$. 
A major part of the problem in certifying neural networks for safety-critical applications is in the fact that traditional analysis methodologies fail to account for changes in systems that occur after the deployment $[1,5,6,8,10,11,12,13]$. Online learning neural networks on the other hand are designed for adaptation (a change) during the operation of the system, i.e. after being deployed into the system. While neural networks in general are considered as inherently difficult for analysis, neural network learning coupled with uncertain adaptive system behavior makes existing traditional analysis techniques practically useless $[1,5,6,8,10,11$, 12, 13]. Additional details relative to the limitation in applicability of traditional analysis approaches for evaluating online adaptive neural networks are discussed later.

The lack of a theoretically valid and practically feasible analysis approach for ensuring correct online adaptive behavior using neural networks remains as one of the critical factors limiting the wider use of neural networks in safety-critical systems. Therefore, there is a growing interest in developing non-conventional analysis procedures suitable for testing and analysis of neural network adaptations of safety-critical systems $[1,5,6,8,10,11,12,13]$.

In the context of the IFCS system, the goal of neural network analysis is to develop a framework for a non-conventional analysis procedure that is suitable for evaluation and testing of the behavior of online learning neural networks. This research goal is a challenge, as it needs to provide analysis techniques for neural networks that are able to adapt during the operation of flight, potentially having direct consequences for the overlaid control system safety. The proposal of this research is to consider stability of online adaptation as a heuristic measure of correctness in the operation of the online adaptive neural networks for the sake of system safety. The idea then is to develop a framework for a non-conventional analysis procedure based on the stability properties of online adaptive neural networks.

Provably stable neural networks guarantee that the learning converges to a stable state within a reasonable amount of time without bifurcating towards instability. Few analytical stability proofs have been given so far for the case of neural network learning $[20,29,38]$. Moreover, most of the existing stability results are confined to static neural network 
architectures such as the feedforward, the multi-layered and the back-propagating neural networks $[25,29,61]$. There is no generalized approach to derive stability results for more complex class of neural networks such as the Kohonen self-organizing maps, the Radial Basis Functions (RBF), or the Dynamic Cell Structures (DCS). An objective of this research is to provide a practical means to answer the following question.

- How to derive a systematic approach for proving stability and convergence properties of dynamic neural networks?

It is observed here that the learning process in dynamic neural networks resembles in many ways, the behavior of dynamical systems $[15,16]$. It is therefore proposed in this research to characterizing the learning behavior in adaptive neural networks in the context of dynamical systems. The idea behind characterize adaptation in neural networks in the context of dynamical systems is that there are well-established dynamical system analysis techniques that could then be applied for analysis of neural networks. One of the foremost of these stability analysis techniques is the Lyapunov stability theory. The interesting feature about Lyapunov's stability analysis is that it can be systematically applied to validate the existence (or nonexistence) of stable-states in a dynamical system [56, 65, 98, 107]. This research therefore, proposes for the extension of the Lyapunov stability theory for analysis of the stability properties of neural networks.

The proposed research plan includes theoretical analysis of the stability properties of neural networks using Lyapunov's second method, also called Lyapunov's direct method. In the context of the online learning in the IFCS system, these stability results can guarantee that the state of online learning converges within a reasonable amount of time to a stable state when exposed to specific representatives of data under certain learning conditions. This means that the analytical stability analysis by itself cannot always guarantee the robustness of the adaptive learning component especially in a rapidly changing operational environment of the adaptive system. The obvious question then is the following. 
- During online adaptation, the neural networks may encounter varying representatives of data that may lead to unstable learning conditions. How to ensure a stable online adaptation for learning conditions that fall beyond the scope of the theoretical stability analysis?

The proposed research calls for the design of an online stability monitoring that can detect atypical behavior of evolving learning states in the online adaptation during the operation of the adaptive system under stressed operating conditions. The availability of online stability monitoring system in such cases for determining if (and when) the neural network converges back to a stable state can significantly enhance safety and reliability of the adaptive system. Figure 1.7 shows the framework of the proposed research approach that consists of two complimentary analysis techniques, the theoretical stability analysis, and online stability monitoring.

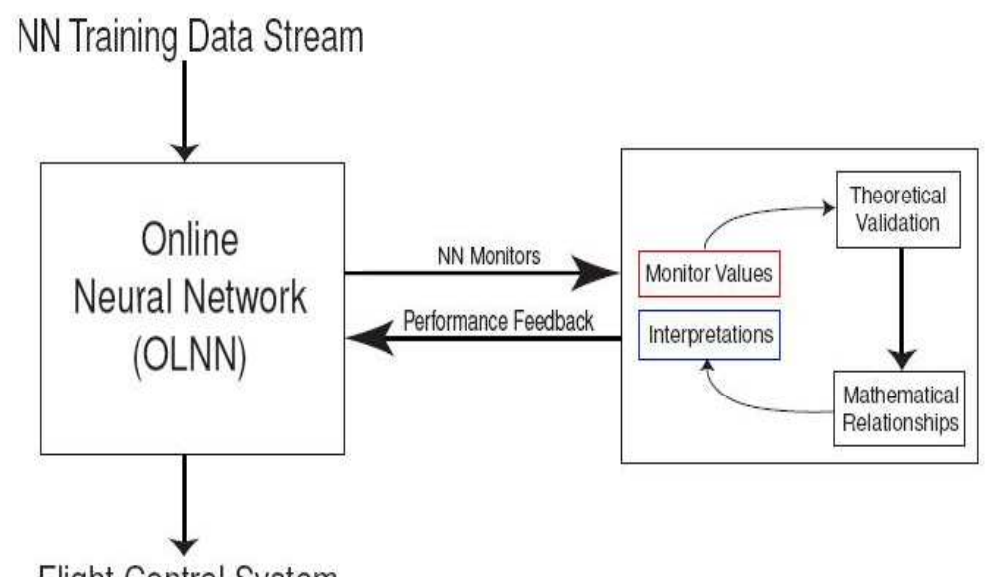

Flight Control System

Figure 1.7 Framework of the proposed non-conventional analysis approach

Some of the major research objectives relative to the analysis of online adaptive neural networks for safety-critical applications that will be of focus in this research are summarized below.

- Provide theoretical assessment in the behavior of adaptive neural networks. 
- Analysis of the stability and convergence properties of neural networks using known stability analysis techniques.

- Determining probabilistic measures of stability in the neural network learning in conditions where an analytical approach fails to apply using the application of online stability monitoring.

- Establish techniques to predict the convergence of stability in neural network learning after subjecting them to data disturbances.

- Overcoming difficulties in neural network implementation by determining the optimal neural network structures and learning parameters.

- Modify the learning process in neural networks for improving the efficiency of online adaptation.

- Generalize the developed techniques for analysis of a general class of neural network architectures. 


\section{Chapter}

\section{Literature Review}

Learning in neural networks can be broadly classified into two classes, supervised, and unsupervised learning. The learning followed in most multi-layer, feedforward, and back propagating neural network architectures involves function interpolation and is regarded as supervised learning $[29,38,61]$. Unsupervised learning refers to learning under nosupervision or no-teacher, i.e. when the training data does not contain information related to the desired values. The goal of unsupervised learning is usually to find regularities or features in the presented data. The adaptation in most self-organizing maps (SOM) including the Kohonen-SOM, the growing cell structures (GCS), and the dynamic cell structures (DCS) can be regarded as unsupervised learning $[17,39]$.

The first section of this chapter describes some details related to the principle operation and convergence properties of static self-organizing maps such as the KohonenSOM. The details relative to the learning process in dynamic self-organizing neural networks such as the dynamic cell structures (DCS) are discussed in the second section. Recall that the DCS is a dynamic self-organizing neural network that realizes online learning in the first generation of the IFCS implementation (Section 1.2, Figure 1.5). The third section of this chapter describes some of the practical limitations for the application of traditional analysis methods for online adaptive neural networks. Recall that this research proposes for the extension of the Lyapunov theory for the stability analysis of online adaptive neural networks (Section 1.3). The final section of the chapter (Section 12.4) describes the notion of stability for dynamical systems according to the Lyapunov stability theory. 


\subsection{Self-Organizing Maps}

Kohonen originally introduced the concept of Self-Organizing Maps (SOMs) in 1981 as biologically inspired adaptive vector quantization algorithms suitable for unsupervised feature extraction [19]. A SOM essentially constructs a structured representation of the presented input data using prototypes, called weight vectors. The weight vectors as a function of neuron coordinates is called a feature map. A scheme for generating topology-preserving feature map is characterized by assigning weight vectors such that nearby data patterns in the input data are mapped to neighboring neurons of the network [24, 25, 37].

\subsubsection{Training Process in Self-Organizing Maps}

For a given input data manifold, the first step in a SOM training is assigning a set of weight vectors for the appropriate neurons of the network. A weight vector is essentially a coordinate position of a neuron in a higher dimensional output space. The weight vectors of neurons in a SOM should not be confused with the weights associated with neurons in a feedforward neural network. In the case of static self-organizing neural networks such as the Kohonen-SOM, the weight vectors are initialized in the form of grid of neurons by random selection of data patterns to form a subset of the given data $[19,22]$. Almost all selforganizing neural networks are based on the concepts of weight vector adaptations whose goal is to preserve the topology of the input data [19, 22].

According to the competitive learning in self-organizing neural networks, a data

pattern $m$, an element of the $n$-dimensional input data manifold, $\mathbf{m} \in \mathbf{M} \subset \mathbf{I} \subset \mathfrak{R}^{n}$ is presented at random to the neural network learning algorithm. The idea behind random presentation of the data patterns for the learning algorithm is to make the learning less susceptible to local minima. For each randomly drawn data pattern, $\mathbf{m} \in \mathbf{M} \subset \mathbf{I} \subset \mathfrak{R}^{n}$, the winning neuron is determined according to an activation function. The neuron with the maximum activation, or the neuron that closely resembles the input pattern $m$, becomes the winning neuron. In most cases, the Euclidean distance between the input data pattern and the 
weight vectors of the neurons serves as a measure of activation. In which case, the winning neuron is the neuron that is closer to $m$ than any other neuron of the network. This neuron is sometimes called the best matching unit (bmu).

Consider a neural network with $N$ neurons projected in a $k$-dimensional output space $\mathbf{O} \subset \mathfrak{R}^{k}$, where the output space is usually of a lower dimension than the input space $\mathbf{I} \subset \mathfrak{R}^{n}, k<n$. The following equation describes the bmu relation.

$$
\left\|\mathbf{w}_{b m u(\mathbf{m})}-\mathbf{m}\right\|=\min _{i}\left\{\left\|\mathbf{w}_{i}-\mathbf{m}\right\|\right\} \quad \forall i \in\{1,2 \ldots \mathrm{N}\}
$$

In a SOM training algorithm, the weight vectors of the winning neurons and the neurons that are connected to the winning neurons (neighbors) get updated. For static neural networks like the Kohonen-SOM, the neighborhood relation is predefined on the competition layer, and remains constant throughout the learning process. The neighborhood relation is a crucial part of the SOM training as it determines what other neurons besides the winning neurons require weight vector update. In a static-SOM, the neighborhood relation is usually represented in the form of a two-dimensional topology. Figure 2.1 and Figure 2.2 show pictures of commonly used neighborhood topologies, a grid topology (Figure 2.1), and a hexagonal topology (Figure 2.2). The weight vectors of all neurons that lie in the competition layer within a certain radius around the winning neurons with respect to the grid structure are considered as neighbors. The strength of the weight vector adaptation may vary depending on the neighborhood relation, $h(\operatorname{bmu}(m), i)$. The weight vectors of all neurons are updated using the following update rule. For additional details on the neighborhood relation including the type of functions used as a neighborhood-kernel, the reader is referred to [19, 22].

$$
\mathbf{w}_{i}(t+1)=\mathbf{w}_{i}(t)+\varepsilon \mathrm{h}(b m u(\mathbf{m}), i)\left(\mathbf{m}-\mathbf{w}_{i}(t)\right) \quad \forall i \in\{1,2 \ldots \mathrm{N}\}
$$

In the Equation (2.8), $0<\varepsilon<1$ is the learning rate, and $\mathrm{h}(b m u(\mathbf{m}), i)$ is the neighborhood function. For most cases, the function $\mathrm{h}(b m u(\mathbf{m}), i)$ has a value of 1 when $\operatorname{bmu}(\mathbf{m}) \equiv i$ and decreases monotonically with increasing distance between $i$ and 
$\operatorname{bmu}(\mathrm{m})$. The most commonly preferred neighborhood function is the bell-shaped Gaussian function,

$$
\mathrm{h}(b m u(\mathbf{m}), i)=\exp \left(-\frac{\left\|r_{b m u(\mathbf{m})}-r_{i}\right\|}{2 \sigma^{2}(t)}\right)
$$

In the Equation (2.9), $r_{b m u(\mathbf{m})}, r_{i}$ represents the locations of the neurons $b m u(m)$ and $i$ in the lattice of Figure 2.1 and Figure 2.2 and $\sigma(t)$ represents the width of the kernel that is usually decreasing over time.

Figure 2.3 and Figure 2.4 show the neighborhood relation in a static-SOM as a parametric function of $\sigma, r_{b m u}$, and $r_{i}$. It can be observed from Figure 2.3 and Figure 2.4 that when $\operatorname{bmu}(\mathbf{m}) \equiv i$, the neighborhood function becomes $\mathrm{h}(\operatorname{bmu}(\mathbf{m}), i)=1$, and then decreases monotonically with increasing distance between $i$ and $b m u(m)$.

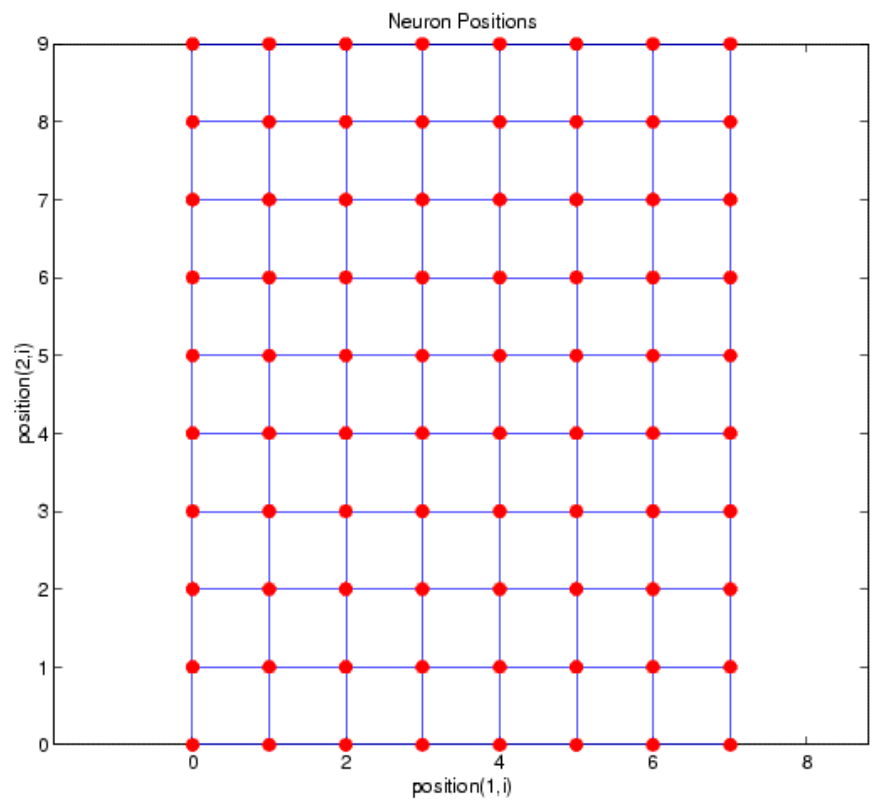

Figure 2.1 A grid neighborhood topology 


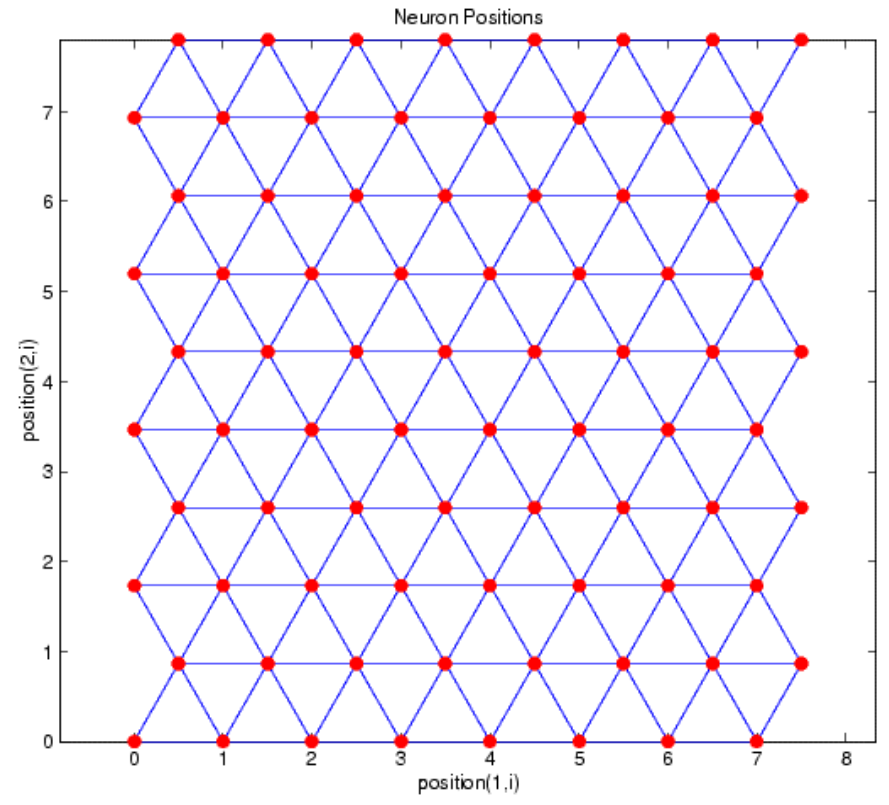

Figure 2.2 A hexagonal neighborhood topology

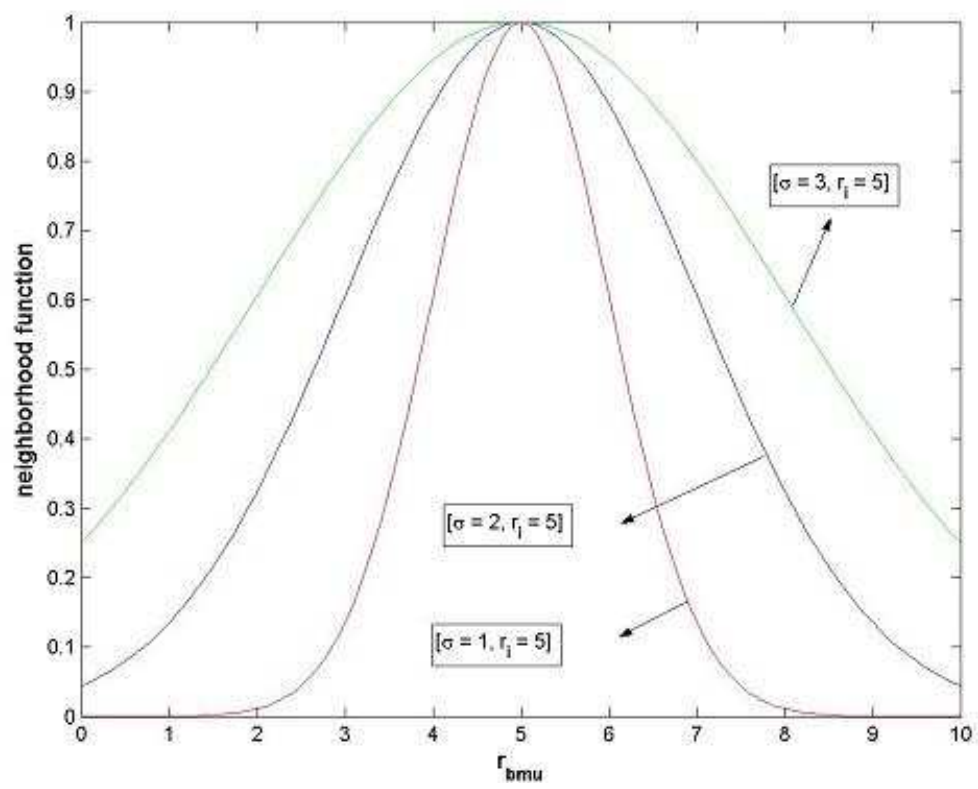

Figure 2.3 Gaussian neighborhood kernel-1 of a SOM 


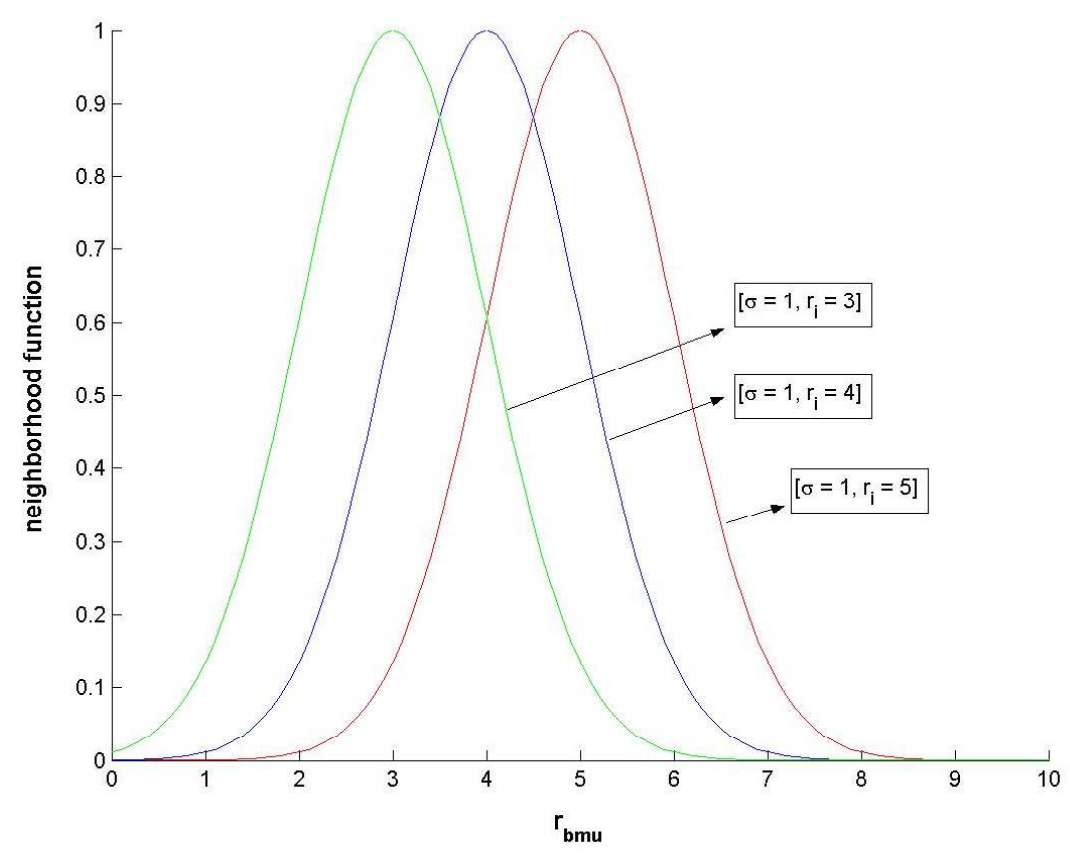

Figure 2.4 Gaussian neighborhood kernel-2 of a SOM

\subsubsection{Convergence Properties of Self-Organizing Maps}

The use of self-organizing maps in safety-critical applications including aircraft control, network computing, and biomedical applications has increased over the years. A general theory describing the learning process in a SOM is, however, still pending $[21,23,29,37,38]$. A major problem in understanding learning in a SOM lies in the stochastic and nondeterministic nature of learning. As a result, SOMs are considered highly resistant for convergence analysis using ordinary differential equations, ODE methods. Even to this day, it is not known if the SOM algorithms follow a stochastic gradient descent on some potential function of the algorithms [21, 23, 26, 27]. So far, Cottrell et al. are the only ones to present a detailed theory for convergence and stability of SOM training following an optimization of a clear energy function [23]. However, their theory and results are significantly restricted to SOM mapping from a one-dimensional data using an open chain (an array) of neurons. Even for the proven case of a simple one-dimensional SOM learning, there is no stochastic gradient 
descent on a single energy function. A set of energy-functions are defined, one for each neuron [23].

There is no general theory in existence for the typically considered high-dimensional situation where the necessary factorization into a product of one-dimensional problems cannot be ensured [27, 28]. Ritter et al. provided some analysis of convergence for higher dimensional learning in the SOM [28]. Heskes proposes modifications of the original Kohonen SOM algorithm to generally obtain cost functions based on the SOM training [27]. It seems that in the case of learning in higher dimensions, a true absorbing state does not exist, and therefore constructing a proof for asymptotic convergence is not likely [27]. While these are the complications in proving convergence for static self-organizing maps, the problem worsens for analysis of stability and convergence properties of dynamic self-organizing neural networks such as the growing cell structures or the DCS. These neural networks are considered as more complex neural network learning paradigms.

There are two major drawbacks relative to the use of static-SOMs for feature extraction. These drawbacks lead to the advent of dynamic self-organizing maps such as the neural GAS, the growing cell structures (GCS), and the dynamic cell structures (DCS) [17, 18, $30,35]$. These drawbacks are briefly discussed in this section. It should be realized that the drawbacks that are observed in static-SOMs serve as the motivation for the development of dynamic-SOMs. Recall that the DCS is a dynamic-SOM that realizes online learning in the first generation of the IFCS implementation (Section 1.2, Figure 1.5).

For any given input data manifold, the first step in a SOM training is assigning a set of weight vectors for the appropriate neurons of the network. In the case of the Kohonen-SOM, the weight vectors are initialized in the form of a grid of neurons obtained by random selection of a subset of the training data (Figure 2.1 and Figure 2.2). However, it was determined by Fritzke et al. that initializing weight vectors in this manner does not necessarily generate an optimal network topology [34]. It was also demonstrated by Fritzke et al. that it is not always possible to determine the optimal initial number of neurons needed for the generation of a topology-preserving feature map especially when the data characteristics are unknown or 
change over time [31, 34]. To overcome this difficulty, the concept of growing self-organizing neural networks (neural gas, growing cell structure) has been introduced [30, 35]. These neural networks essentially begin with a small number of neurons and gradually increase the number of neurons until there are enough neurons in the network to generate a topologypreserving feature map. It is shown that the growing self-organizing neural networks are suitable for following non-stationary data distributions [30, 35].

Later, the idea of growing self-organizing neural networks was modified by Bruske et al., who introduced the concept of dynamic self-organizing neural networks in the form of the DCS $[17,18]$. It has been demonstrated that dynamic neural networks such as the DCS are able to capture the topology of the input data more effectively, and do not require any prespecification of the input data characteristics $[17,18,36,37]$. A dynamic-SOM such as the DCS initializes the network by assigning two weight vectors over two randomly selected data patterns from the input data manifold. The neural network learning algorithm gradually introduces additional neurons into appropriate regions of the network in order to generate a map that faithfully preserves the topology of the input data $[17,18,30,35]$.

In general, a static-SOM is able to extract features from a wide range of high dimensional data configurations, and is capable of projecting the extracted features onto a 2dimensional plane. However, for certain configurations of data, the Kohonen-SOM cannot generate a topologically correct representation of the input data manifold. As an example, Figure 2.5 shows an example of an input data manifold comprising of discrete data clusters. A representation of the discrete input data clusters using Kohonen-SOM with a 10x10 grid neighborhood topology is shown in Figure 2.6. The example demonstrates that by fixing the neighborhood relation (in this case, using a grid topology), the SOM restricts its ability to generate a topologically correct network representation of the given input data. Ingeneral, it can be shown that by fixing the neighborhood relation (grid or hexagonal topology), the SOM severely restricts its ability to generate a topology-preserving feature map of the given input data. 
While the use of static-SOMs is acceptable for feature extraction by dimensional reduction, it may not be practical for function approximation of discontinues, high-dimensional data sets. To overcome this difficulty of dimensional and topological restrictions, dynamicSOMs such as the neural gas, the growing cell structures, and the dynamic cell structures (DCS) have been proposed [17, 18, 30,35]. An important feature of the dynamic-SOMs is that the neighborhood relation is not pre-specified, but it rather evolves during the neural network self-organization process.

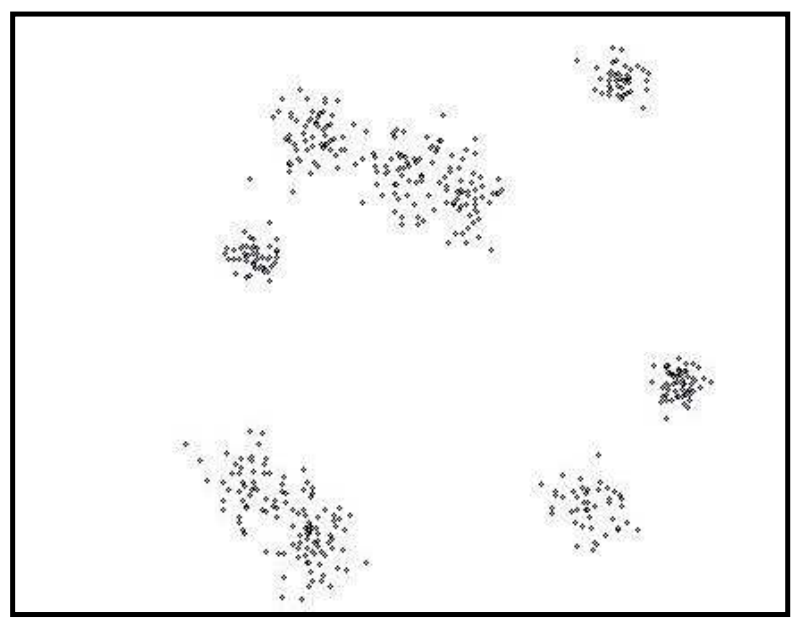

Figure 2.5 NN training discrete data clusters

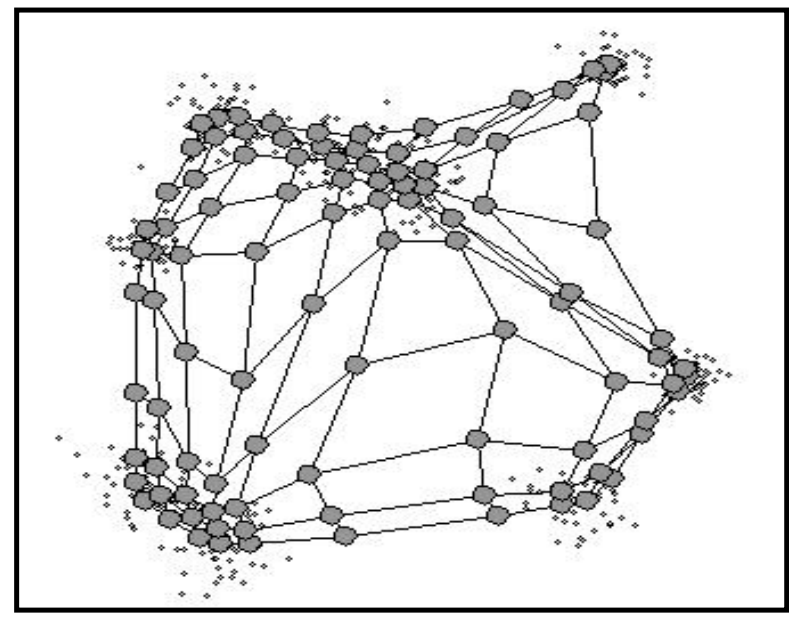

Figure 2.6 Kohonen-SOM after 1700 learning cycles 


\subsection{Dynamic Cell Structures Neural Networks}

Jorg Bruske and Gerald Sommer introduced the Dynamic Cell Structure (DCS) in 1994 as a dynamic self-organizing neural network capable of generating topology-preserving feature maps $[17,18]$. The learning process in the DCS that leads to the generation of a topology- preserving feature map is motivated by prior work on growing self-organizing maps by Fritzke and Martinetz [19, 21, 28, 35]. The DCS neural network represents a dynamic selforganizing neural network that is capable of generating topology preserving feature maps from both, stationary and non-stationary data distributions. Recall that the DCS realizes online adaptation in the first generation of the IFCS implementation by serving as the online learning neural network (refer to Section 1.2, Figure 1.5) $[14,40,49,50]$. This section describes details relative to the learning process in the DCS. Some of the material presented in this section will be used in the following chapters for characterizing the DCS neural network in the context of a dynamical system.

The DCS utilizes a radial basis function (RBF) and an additional layer of laterally connected neurons to construct a network of representative neurons for a given data set. Consider a DCS neural network consisting of $\mathrm{N}$ neurons that is training from an input manifold, $\mathbf{M} \subset \mathbf{I} \subset \mathfrak{R}^{n}$ and projects the neural network map in the output space $\mathbf{O} \subset \mathfrak{R}^{n}$. Note that unlike the Kohonen-SOM where the dimensionality of output space is usually less than that of the input space, the input and output space in the DCS are of the same dimensionality. This is because our interest is within adaptive vector quantization using the DCS that does not involve dimension reduction.

\section{Definition 2-1 Best Matching Unit (bmu)}

The best matching unit (bmu) for $m$, a data pattern of the input manifold, $M$, is a neuron of the DCS network, $i=b m u(\mathbf{m}), \quad i \in\{1,2 \ldots N\}$ which is closest (in terms of Euclidean distance) to the input element than any other neuron of the network.

Considering $\mathbf{w}_{b m u(\mathbf{m})} \in \mathbf{O} \subset \mathfrak{R}^{n}$ as the weight vector of the best matching unit of $m$, the $b m u$ relation is given as follows. 


$$
\left\|\mathbf{w}_{\text {bmu }(\mathbf{m})}-\mathbf{m}\right\|=\min _{i}\left\{\left\|\mathbf{w}_{i}-\mathbf{m}\right\|\right\} \quad \forall i \in\{1,2 \ldots \mathrm{N}\}
$$

\section{Definition 2-2 Second Best Matching Unit (sbu)}

The second best matching unit (sbu) for $m$, a data pattern of the input manifold, $M$, is a neuron of the DCS network, $i=\operatorname{sbu}(\mathrm{m}) \quad i \in\{1,2, \mathrm{~K}, N\}, i \neq b m u(\mathrm{~m})$ which is second closest (closest being the $B M U)$ to the input element than any other neuron of the network.

Considering $\mathbf{w}_{s b u(\mathbf{m})} \in \mathbf{O} \subset \mathfrak{R}^{n}$ as the weight vector of the second best matching unit of $m$, the sbu relation is given as follows.

$$
\left\|\mathbf{w}_{s b u(\mathbf{m})}-\mathbf{m}\right\|=\min _{i}\left\{\left\|\mathbf{w}_{i}-\mathbf{m}\right\|\right\} \quad \mid \mathbf{w}_{s b u(\mathbf{m})} \neq \mathbf{w}_{b m u(\mathbf{m})} \quad \forall i \in\{1,2 \ldots \mathbf{N}\}
$$

Note that in the DCS, the lateral connections between neurons are symmetric and bounded in nature, $\forall i, j \in\{1,2 \mathrm{~K} N\} \mid i \neq j, 0<c_{i j} \equiv c_{j i}<1$. If two neurons are connected by non-zero connection strength, then they are considered neighbors, $n b r$. The following definition provides a mathematical formulation for the neighborhood relationship in the DCS.

\section{Definition 2-3 Neighbors (NBR)}

Two neurons $i, j \in\{1,2 \ldots N\}$ of the DCS network with weight vectors, $\mathbf{w}_{i}, \mathbf{w}_{j} \in \mathbf{O} \subset \mathfrak{R}^{n}$ are considered neighbors if they are connected to each other by non-zero lateral connection strength. In this case, the neuron $i$ is considered a member of the neighborhood of the neuron $j$, and vice versa.

$$
\forall i, j \in\{1,2, \mathrm{~K}, N\} \text {, if } c_{i j}=c_{j i}>0 \text {, then } j \in\{n b r(i)\} \text { and } i \in\{n b r(j)\}
$$

\subsubsection{The Competitive Hebb Rule}

Unlike static-SOMs where the neighborhood relation is a predefined constant, the competitive layer in the DCS adapts to the topology of the input data. The lateral connection strengths between neurons of a DCS network are updated using the competitive Hebb rule $(\mathrm{CHR})$. Updating lateral connections between neurons using the CHR generates a network 
map where the input data patterns that are nearby are mapped using neighboring neurons. Thereby, the CHR avoids any restrictions of the network topology [17, 18, 37].

The CHR operates by first setting the connection between the $b m u$ and the sbu units to highest possible connection strength of value 1 . The connection strengths of other neurons connected to the bmu are decremented using decay constant, $\alpha$. If any of these connections drops below a predefined threshold, $\theta$, then such connections are reset to zero. All other connections of the neural network remain unaltered. The competitive Hebb rule is summarized in the following manner.

$$
c_{i j}(t+1)= \begin{cases}1 & \text { if }(i=b m u) \wedge(j=s b u) \\ 0 & \text { if }(i=b m u) \wedge(j \neq s b u) \wedge(j \in n b r) \wedge\left(c_{i j}<\theta\right) \\ \alpha c_{i j}(t) & \text { if }(i=b m u) \wedge(j \neq s b u) \wedge(j \in n b r) \wedge\left(c_{i j} \geq \theta\right) \\ c_{i j}(t) & \text { otherwise }\end{cases}
$$

Application of the CHR before any other adjustment ensures that the sbu is a neighbor of the bmu within that learning cycle. It was shown by Martinetz et al. that neural networks that utilize the $\mathrm{CHR}$ for updating lateral connections between the neurons are capable of generating topology-preserving feature mappings [37].

\subsubsection{A Kohonen-Like Rule}

It is crucial to realize that the weight vectors of a neural network be updated in a manner that preserves the geometry of the input data. The DCS uses a Kohonen-like rule for adapting the weight vectors of neurons in its network. The adaptation in the DCS is such that the weight vectors gradually move closer to the presented data patterns.

Let $\mathbf{m} \in \mathbf{M} \subset \mathbf{I} \subset \mathfrak{R}^{n}$ be a data pattern of a given input data manifold [17, 18]. Over any training cycle, consider $\Delta \mathbf{w}_{i}=\mathbf{w}_{i}(t+1)-\mathbf{w}_{i}(t), \forall i \in\{1,2 \mathrm{~K} N\}$ as the adaptation of the weight vectors in a DCS neural network. The Kohonen-like rule that is followed in the DCS training algorithm is 


$$
\Delta \mathbf{w}_{i}= \begin{cases}\varepsilon_{\mathrm{bmu}}\left(\mathbf{m}-\mathbf{w}_{i}\right) & \text { if } i \equiv \operatorname{bmu}(\mathbf{m}) \\ \mathcal{E}_{\mathrm{nbr}}\left(\mathbf{m}-\mathbf{w}_{i}\right) & \text { if } i \in\{\operatorname{nbr}(\operatorname{bmu}(\mathbf{m}))\} \\ 0 & \text { otherwise }\end{cases}
$$

In the above equation, $\varepsilon_{b m u}, \varepsilon_{n b r}$ are predefined constants known as the learning rates that define the momentum of the weight adaptation process. Adapting the weight vectors of neurons using a Kohonen-like rule as given in Equation (2.14), and updating lateral connections between neurons using the Hebb rule as given in Equation (2.13) is shown to generate network representations that preserve the features of given input data manifolds [37].

\subsubsection{NN Growing Process in the DCS}

Unlike a static self-organizing neural network, the DCS has the ability to grow (or shrink) the neural network map. A local error measure, namely resource value is associated with every neuron of the network. The resource information is used in the neural network growing process. In most cases, the Euclidean distance between the data patterns and the corresponding best matching $(b m u)$ units serve as a measure of the resource values. If it is needed, a new neuron is introduced into the DCS network in a region between the neurons that have the highest resource value and their connected neighbors with the second highest resource value.

\subsubsection{DCS NN Training Algorithm}

It was discussed previously that neural network such as the DCS that use a Kohonenlike adaptation to adapt the weight vectors, and the $\mathrm{CHR}$ to update the lateral connections between neurons generate network representations that preserve the features of the input data [37]. The DCS relies heavily on Kohonen-like rule and $\mathrm{CHR}$ that are given in Equations (2.13) and (2.14). The DCS training algorithm is allowed to train on a given input data manifold until the cumulative network error reaches a pre-specified error level. Figure 2.7 shows the control flow diagram of the online learning DCS algorithm. 
As shown in Figure 2.7, for every randomly drawn data pattern of the given input manifold, the DCS algorithm first selects the $b m u$ and the sbu units according to Equations (2.10) and (2.11). The lateral connections between the neurons are updated using the CHR according to Equation (2.13). The weight vectors of the $b m u$ and its neighbors (nbr) are updated accordingly using a Kohonen-like adaptation rule as in Equation (2.14). The resource value of the bmu is updated. If needed, additional neurons are introduced into the DCS network using a neural network growing strategy. Decrementing the resource values of neurons using decay constant prevents the resource values from growing out of bounds. For additional details of the DCS training algorithm, the reader is referred to the work of Bruske and Sommer $[17,18]$.

\subsubsection{DCS NN Output}

When the DCS neural network is presented with a test input, $\mathbf{m}^{*} \in \mathbf{I} \subset \mathfrak{R}^{n}$ the $b m u$ and the set of neurons that are connected to the bmu (neighbors of the $b m u$ ) according to Equations (2.10) and (2.12). A neighbor of the bmu that is closest to the bmu is selected. Note that due to the stochastic nature of the DCS learning, the closest neighbor of the bmu need not always be the sbu. A linear interpolation between the bmu and its closest connected neighbor is performed to generate an estimate of the output value,

$$
\mathbf{y}\left(\mathbf{m}^{*}, \mathbf{w}_{b m u\left(\mathbf{m}^{*}\right)}, \mathbf{w}_{n b r\left(b m u\left(\mathbf{m}^{*}\right)\right)}\right) \in \mathbf{O} \subset \mathfrak{R}^{n}
$$

It is evident from the above equation that the DCS output is a function of the current state of the neural network, and the location of the test input, $\mathbf{m}^{*} \in \mathbf{I} \subset \mathfrak{R}^{n}$ in the Cartesian plane. If the bmu is not connected to any other neuron of the DCS network, then the output is based solely on the location of the bmu. It is obvious from the DCS output function that the output produced by a DCS network with no neurons is zero. 


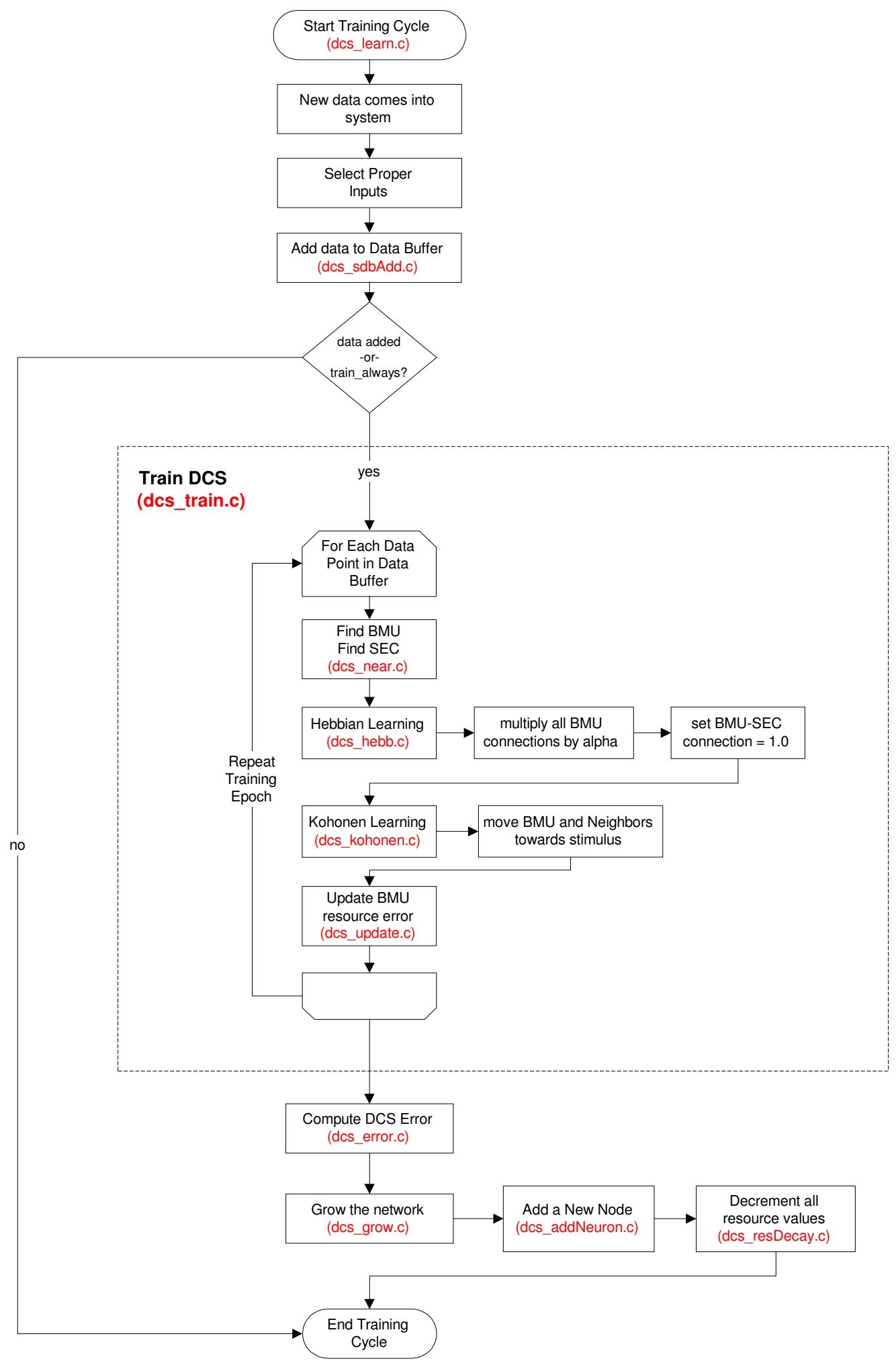

Figure 2.7 Control Flow Diagram of the Online Learning DCS Neural Network 


\subsection{Limitations of Traditional NN Analysis Methods}

When a neural network performs online adaptation, its behavior may have direct consequence on the overlaid adaptive system. Therefore, it is necessary to understand the nature of adaptation in neural networks before their actual deployment into a safety-critical system $[1,5,6,10,14,69,73,80,82]$. The question then is:

- How to derive a systematic analysis procedure for ensuring correct neural network behavior during its online adaptation in safety-critical systems?

A major part of the problem in certifying neural networks for safety-critical applications is the fact that traditional analysis methodologies fail to account for changes in a system that may occur after deployment. Online learning neural networks, on the other hand, are designed for adaptation (a change) during the operation of the system, i.e. after being deployed into the system. Therefore, it is not practical to apply traditional analysis techniques for ensuring correct online adaptation using neural networks. This section describes some details related to the limitations in the application of traditional analysis techniques for ensuring correct online neural network adaptation.

\subsection{1 $\quad$ Formal Methods}

Formal methods refer to the application of techniques from formal logic and discrete mathematics for the design and construction of software. Formal methods of rule extraction, rule initialization, and rule insertion (refinement) are some of the techniques being researched upon for analysis of neural networks [7, 8].

Continuous adaptation demands frequent extraction and/or update of rules. This is a major challenge for rule extraction as online learning neural networks are designed for adaptation during the operation of the system. A limitation that exists within rule extraction is that the algorithms and tools developed for the rule extraction work only for the specific architectures of the neural networks for which they are designed [7]. Through rule initialization, the neural network is given a starting point from which it is expected to adapt. Though this technique is intended to improve confidence in the neural network behavior, setting up a 
starting point through rule initialization could lead to a constrained learning regime $[7,8]$. Rule insertion can be performed periodically, while in operation or offline, to steer a dynamic neural network towards a desired operational regime $[7,8]$. However, it is in general hard to determine the desired operational regime for unsupervised adaptation such as online learning neural networks.

For safety-critical applications such as the IFCS system, a rigorous formal method is required in order to ensure correct (and possibly safe) neural network behavior. However, a known issue with the application of formal methods is that the more rigorous the formal method, the more computational effort and skill required [7, 10]. For additional details regarding the research applications of formal methods for neural network analysis, the reader is referred to $[7,8]$.

\subsubsection{Testing Methods}

Testing a system using a subset of the training data is a commonly used software testing methodology to verify whether the learning mechanism, in our case the neural network, has adequately captured the input domain $[1,2,5,9,12,14]$. A difficult step in testing adaptive neural networks is the choice of test cases to probe the behavior of the system. In complex adaptive systems like the IFCS, the non-deterministic nature of adaptation makes it practically impossible to test the neural network for all domains of data [5, 9, 12, 14]. Moreover, testing methodologies require an additional overhead of testing-data generation schemes that makes them less practical for analysis of neural networks used in adaptive

systems $[5,9]$. While traditional testing methodologies prove adequate for acceptance of neural networks in standard applications, they are inadequate for safety-critical applications $[1,8,10,13]$.

\subsubsection{Cross Validation}

The concept of cross validation centers on combining diverse neural networks into an ensemble. The output from the component neural networks may then be checked against one 
another to affirm validity and appropriateness [8]. Cross validation is used in neural network analysis for developing differing solutions for a single input domain and then comparing and combining these solutions against each other to obtain a single result [2].

While results indicate that using ensembles of neural networks increases the performance over a single neural network, redundancy alone does not ensure improved performance. Firstly, one must determine the kind of diversity that may lead to an improved performance. Secondly, one has to determine the best way of creating sets of neural networks that show this kind of diversity [8]. The idea of using a group of neural networks to validate a neural network can seem questionable. Moreover, a combination of outputs from several neural networks is only useful if they disagree on something. Clearly there is no more information to be gained from combining several different neural networks than from a single neural network [2].

While neural networks in general are considered as inherently difficult to analyze, neural network learning coupled with uncertain adaptive system behavior makes existing traditional analysis approaches practically useless $[1,8,10,13]$. Limitations in applicability of traditional analysis techniques for ensuring correct neural network behavior can obstruct the provision of a safe and reliable platform for online adaptation in safety-critical systems. Therefore, there is a growing interest in developing sophisticated, non-conventional techniques for testing and analysis of online adaptation using neural networks [10, 13].

In the context of the IFCS system, the goal is to develop a framework for a nonconventional analysis approach suitable for online learning neural networks such as the DCS (the OLNN shown in Figure 1.5 in Chapter 1). This research goal is a challenge, as it needs to provide analysis techniques for online learning neural networks that are able to adapt during the operation of the flight, potentially having direct consequences for the overlaid flight system safety. 


\section{$2.4 \quad$ Lyapunov Stability Theory}

It is observed in the research that the process of adaptation in online learning neural networks resembles in many ways the behavior of dynamical systems $[15,16]$. Based on the interpretation of neural networks as a dynamical system, the proposal of this research is to consider the stability and convergence of online adaptation as heuristic measures of correctness for the sake of system safety. The idea is to first characterize the process of online adaptation in neural networks in the context of dynamical systems, and then apply available techniques for stability analysis of dynamical systems to neural networks. One of the foremost of the stability analysis techniques is the Lyapunov stability analysis. The interesting feature about Lyapunov stability analysis is that it can be systematically applied to validate the existence (or inexistence) of stable states in dynamical systems [56, 65].

Based on the proposal of considering stability and convergence as heuristic measures of correctness in neural network learning, the research objective is to develop a framework for a non-conventional analysis procedure suitable for evaluation and testing of non-deterministic neural networks. Since the concept of applying Lyapunov theory for stability analysis of neural networks is a relatively novel analysis approach, this section provides the reader with a basic understanding of the fundament concepts of stability according to the Lyapunov's theory. For additional details on the use of Lyapunov's theory for stability analysis of dynamical systems, the reader is referred to $[57,59,62,65,98,107]$. Stability in Dynamical Systems

A dynamical system is an evolution rule on a set of states, the phase space, defined as a function of time as a parameter. The evolution rule can be deterministic or stochastic, depending on the nature of the system. A system is deterministic if for each state in the phase space there is a unique consequent, i.e., the evolution rule is a function taking a given state a unique, subsequent state $[62,65]$. Stochastic systems are non-deterministic: a standard example is the idealized coin toss. The process of adaptation in neural networks evolves over time in an unpredictable manner, and therefore is mostly stochastic and can be characterized in context of dynamical systems. The mathematical theory of stability analysis deals with 
validating the existence (or inexistence) of stable states within a dynamical system using rigorous mathematical derivations and analysis techniques. Stability of a dynamical system is usually defined in terms of the system's equilibrium point and not on the system. A formal definition of equilibrium point for dynamical systems is provided here.

\section{Definition 2-4 Equilibrium Point}

Consider a nonlinear time-invariant system, $\mathbf{k}=f(\mathbf{x})$, where $\mathbf{x}=\left[x_{1}, x_{2} \mathrm{~K} x_{m}\right]^{T}$ are the states of the system. Let $f: \Omega \rightarrow \Re^{n}$ be a continuously differentiable function, and $\Omega \subseteq \mathfrak{R}^{n}$ is a subset of Euclidean space. A point $\mathbf{x}_{e} \ni \Omega$ is an equilibrium point of the system if $f\left(\mathbf{x}_{e}\right)=0$.

It should be noted that $\mathbf{x}_{e}$ is an equilibrium point implies that $\mathbf{x}(t)=\mathbf{x}_{e}$ is a trajectory of the system. Considering the origin as the equilibrium point of $\&=f(\mathbf{x})$, i.e. $\mathbf{x}_{e}=0$, the following definitions introduce the notion of stability of dynamical systems. For further details on the concept of stability of dynamical systems, the reader is referred to $[52,54,57,59,62$, $65,98,107]$.

Definition 2-5 Local Stability

If for every $\mathcal{\varepsilon}>0$, and $t_{o} \in \mathfrak{R}$, there exists $\delta\left(\varepsilon, t_{o}\right)>0$ such that if $\left|\mathbf{x}\left(t_{0}\right)\right|<\delta$ then $|\mathbf{x}(t)|<\mathcal{E}$ for all $t \geq t_{0}$, then the equilibrium point $\mathbf{x}_{e}=0$ of the system $\mathbf{l}=f(\mathbf{x})$ is said to be locally stable at time $t_{o}$.

The concept of stability given in Definition 2-5 is illustrated in Figure 2.8, where a system's trajectory starting close to the equilibrium is shown to remain arbitrarily close. Note that in the case of Figure 2.8, the equilibrium point is the origin, $\mathbf{x}_{e}=(0,0)$. An equilibrium state is unstable if the above condition is not satisfied. In linear systems, instability means approaching different trajectories arbitrarily close in any given time. However, this is not the case with nonlinear systems, which makes nonlinear stability analysis a challenge. 


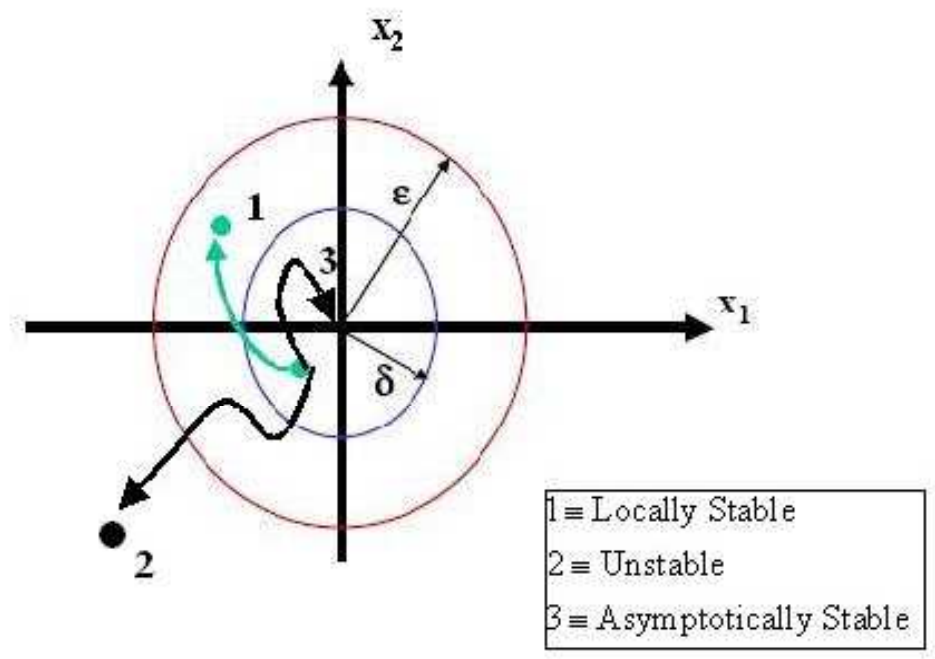

Figure 2.8 Illustration of local stability concept of a dynamic system

\section{Definition 2-6 Local Asymptotic Stability}

If the equilibrium point, $\mathbf{x}_{e}=0$ is locally stable and if for every $t_{o} \in \mathfrak{R}$, there exists $\delta\left(t_{o}\right)>0$ such that if $\left|\mathbf{x}\left(t_{0}\right)\right|<\delta$ then $|\mathbf{x}(t)| \rightarrow 0$ as $t \rightarrow \infty$, then the equilibrium point $\mathbf{x}_{e}=0$ is said to be locally asymptotically stable.

In essence, local asymptotic stability (Definition 2-6) implies that a system trajectory starting sufficiently close to the equilibrium point will eventually approach the equilibrium point. The equilibrium point is conventionally chosen as the origin

\section{Definition 2-7 Global Asymptotic Stability}

If the equilibrium point $\mathbf{x}_{e}=0$ is locally stable and if for every initial condition, $x\left(t_{o}\right),|\mathbf{x}(t)| \rightarrow 0$ as $t \rightarrow \infty$, then the equilibrium point $\mathbf{x}_{e}=0$ is said to be globally asymptotically stable.

In essence, global asymptotic stability (Definition 2-7) implies that if the asymptotic stability condition holds good for any initial condition, then it can be said that the equilibrium point of the dynamical system is global asymptotically stable.

Most of the previously discussed notions of stability are based on the solution (equilibrium point) for the difference equation governing the system dynamics. In general, it is 
inherently difficult to solve higher order difference equations, and there is no guarantee for the existence of a solution for certain higher order nonlinear difference equations [62]. This difficulty in finding a solution for the difference equations can be overcome by the construction of a Lyapunov function $[57,59,62,65,98,107]$. A unique feature about Lyapunov function based stability analysis is that one establishes conclusions about trajectories of a system without actually finding the trajectories i.e., solving the difference equations.

\section{Definition 2-8 Lyapunov Function}

If $V: \Re^{n} \rightarrow \Re$ is continually differentiable and locally positive definite function around $\mathbf{x}_{e}=0$ such

that all sublevels of $V$ are bounded and $\&(\mathbf{x}) \leq 0 \quad \forall \mathbf{x}$, then all trajectories of the system $\stackrel{\mathrm{g}}{\mathbf{x}}=f(\mathbf{x})$ are bounded and $V$ is called the Lyapunov function.

The relevant result of Lyapunov stability theory in terms of a Lyapunov function is given in the following mathematical derivation.

\section{Theorem 2.1 Lyapunov Stability}

If there exists a Lyapunov function for the system, $\quad \&=f(\mathbf{x})$, then $\mathbf{x}_{e}=0$ is said to be a stable equilibrium point in the sense of Lyapunov.

Proof For a detailed mathematical proof, the reader is referred to [52, 54, 62, 100, 102, 106].

According to Lyapunov theory, a system is said to be stable near a given solution if all solutions of the state that begin nearby, end up nearby. A good measure representing the notion of "nearby" is the size of the domain of the Lyapunov function by a Lyapunov function, $V$ over the states of the system.

By constructing a Lyapunov function one can guarantee that all trajectories of the system converge to a stable state, i.e. if they lie in the domain of the definition of the constructed Lyapunov function. The Lyapunov function should be constructed keeping in mind that it needs be a scalar, $V: \mathfrak{R} \times D \rightarrow \mathfrak{R}$, and should be non-increasing over the trajectories of the state space (at least negative semi-definite) $[63,64]$. This is required in order to ensure that all limit points of any trajectory are stationary. A strict Lyapunov function should force 
every trajectory to asymptotically approach equilibrium state [65]. Even for non-strict Lyapunov function, it is possible to guarantee convergence by LaSalle's invariance principle [62, 103].

\section{Theorem 2.2 Asymptotic Stability}

If $\mathbf{x}_{e}=0$ in addition to being Lyapunov stable, $\left.\& \mathbf{x}\right)$ is locally negative definite, then $\mathbf{x}_{e}=0$ is an asymptotically stable equilibrium point.

Proof For a detailed mathematical proof the reader is referred to $[52,54,62,100,102,106]$.

Asymptotic stability adds the property that in a region surrounding a solution of the dynamical system trajectories are approaching this given solution asymptotically.

\section{Theorem 2.3 Global Asymptotic Stability}

If $\mathbf{x}_{e}=0$, in addition to being Lyapunov stable, $\&(\mathbf{x})$ is negative definite in the entire state space and $\lim _{t \rightarrow \infty} V(\mathbf{x})=0$, then $\mathbf{x}_{e}=0$ is a global asymptotically stable equilibrium point.

Proof For a detailed mathematical proof the reader is referred to $[52,54,62,100,102,106]$.

A notable difference between asymptotic and global asymptotic stability is the fact that the later implies that any trajectory beginning at any initial point will converge asymptotically to the given solution, whereas the former implies that only those trajectories that begin in the neighborhood of the solution approach the solution asymptotically. The types of stability defined above have increasing property strength, i.e. Global Asymptotic Stability $\Rightarrow$ Asymptotic Stability $\Rightarrow$ Lyapunov Stability. The reverse implication, however, does not necessarily apply.

Though the concept of Lyapunov stability was originally intended for use in mathematical theory, it can be simplified for use in many practical applications including neural networks [15, 16, 60, 61]. In mechanical systems, a Lyapunov function is considered as an energy-minimizing term and in economy and finance evaluations it is considered as a costminimizing term, and for neural networks, the construction of a Lyapunov function can be based on the error-minimizing states of the neural network learning which will become evident in the following chapters. 


\section{Chapter}

\section{Theoretical Stability Analysis}

The central goal of an adaptive system is to calculate the present state of the system and determine a strategy to drive the system to a desired operating state. From the discussions in Chapters 1 and 2, it is evident that using online adaptive components such as the neural networks significantly enhances the functionality of the adaptive system. It is important to realize that by accommodating for changing dynamics of the system, online adaptive components play a critical role in the functionality of the adaptive system. Therefore, it is necessary to ensure correct behavior of the online adaptive components before their deployment into the actual safety-critical system $[1,4,6,8,10,14]$.

A provably stabilizing online adaptation ensures that the learning in an embedded adaptive component converges to a stable state within a reasonable amount of time without bifurcating towards instability. Therefore, this research proposes a theoretical analysis of the stability properties of online adaptation. In the context of the analysis of online adaptation within the IFCS system, the goal of theoretical stability analysis is to delineate stability boundaries of online adaptation for certain specific domains of adaptive system data using mathematical theories of stability analysis. In the first generation of the IFCS implementation, the online adaptation is realized by the DCS, a dynamic self-organizing neural network [17, $18,40,49,50]$. It is, therefore, necessary to consider the following questions regarding stability analysis of the DCS neural networks.

- How to derive a systematic approach to delineate stability-boundaries of online adaptation in a dynamic neural network such as the DCS?

- To what specific domains of the adaptive system data are the delineated stability boundaries confined?

It is observed as a part of the research that the learning process in neural networks evolves over time in a manner similar to the behavior of dynamical systems $[15,16]$. The idea 
then is to characterize neural network learning in the context of dynamical systems and analyze its stability properties using dynamical system analysis techniques. One of the foremost of the stability analysis techniques that is commonly applied for dynamical systems is Lyapunov stability analysis. The interesting feature about Lyapunov's stability theory is that it can be systematically applied to validate the existence (or inexistence) of stable states in a dynamical system $[57,65]$. The research therefore, proposes for the extension of the Lyapunov's stability theory for analysis of the stability properties of online learning neural networks.

Due to the stochastic nature of learning in dynamic neural networks, the stability analysis, in this chapter, is restricted to NN adaptation from stationary or fixed data manifolds. Learning from a stationary data manifold implies that once a certain data manifold is presented to the online adaptive component (neural network), the configuration of the data remains unchanged throughout the learning process.

\subsection{Learning Dynamics of the DCS}

This sections aims towards characterizing the learning process in neural network in the context of a dynamical system. The first step in this stability analysis is the identification of stable-states involved in the DCS neural network. The learning process involved in the DCS was discussed in Chapter 2, Section 2.2. A state-space representation is a commonly preferred method for representation of the states within a dynamical system. It is important to represent the states of the dynamical system (online learning neural network) using a statespace representation technique as it can prove to be effective during the construction of a Lyapunov function $[56,59,62,64,103,107]$.

Consider D-dimensional input and output manifolds, $\quad \mathbf{C} \subset \mathfrak{R}^{D}$ and $\mathbf{O} \subset \mathfrak{R}^{D}$, respectively. The DCS is a self-organizing neural network consisting of neurons that are positioned in the output space $\mathbf{O} \subset \mathfrak{R}^{D}$ using weight vectors. Consider $N$ as the number of neurons in the DCS network at any time. The weight vectors of the DCS neural network can 
be presented as, $\mathbf{w}_{i} \in \mathbf{O} \subset \mathfrak{R}^{D} \forall i \in\{1,2 \ldots N\}$. The lateral connections between neurons in the DCS are expressed in terms of real constants known as connection strengths, $c_{i j} \equiv c_{j i} \in[0,1] \forall i \neq j i, j \in\{1,2 \ldots N\}$. Unlike feedforward neural networks, the lateral connections between neurons in a self-organizing neural network are not static, but evolve over time $[17,18]$. A D-dimensional DCS neural network consisting of $N$ neurons can, therefore, be represented in the output space $\mathbf{O} \subset \mathfrak{R}^{D}$ using a $D \times N$ position matrix, $\mathbf{W}_{D \times N} \ni \mathbf{w}_{i}$ and a $N \times N$ connection strength matrix, $\mathbf{C}_{N \times N} \ni c_{i j}$. For a given input space $\mathbf{I} \subset \mathfrak{R}^{D}$, the DCS is essentially an undirected graph of $\mathbf{W}_{D \times N}$ and $\mathbf{C}_{N \times N}$ that can be defined in the following manner.

\section{Definition 3-1 DCS Mapping}

For a given input space, $\mathbf{I} \subset \mathfrak{R}^{D}$, the DCS neural network mapping is an undirected graph of $\mathbf{W}_{D \times N}$ and $\mathbf{C}_{N \times N}, \mathrm{G}\left(\mathbf{W}_{D \times N}, \mathbf{C}_{N \times N}\right): \mathbf{I} \subset \mathfrak{R}^{D} \rightarrow \mathbf{O} \subset \mathfrak{R}^{D}$ that can be considered as an $N^{\text {th }}$ order neural network representation of the input manifold, $\mathbf{M} \subset \mathbf{I} \subset \mathfrak{R}^{D}$ in the output space, $\mathbf{O} \subset \mathfrak{R}^{D}$ generated by assigning $N$ neural units using the DCS training algorithm (Section 2.22.2.4).

Based on the information provided in Definition 3-1, the Hebb and a Kohonen-like adaptation rules discussed in Section 2.2, the idea is to provide a state-space representation of the DCS neural network. Let the states of the DCS neural network due to the adaptation of weight vectors using a Kohonen-like rule (refer to Chapter 2) and the Hebb update (refer to Chapter 2) be represented by $\mathbf{x}_{W}$ and $\mathbf{x}_{C}$ respectively. The dynamics of the state changes in the DCS due to the adaptation of weight vectors using a Kohonen-like rule and the Hebb rule can be represented in the following manner.

$$
\begin{aligned}
\frac{\Delta \mathbf{x}_{W}}{\Delta t} & =f_{W}\left(\mathbf{x}_{W}, \mathbf{x}_{C}\right) \\
\frac{\Delta \mathbf{x}_{C}}{\Delta t} & =f_{C}\left(\mathbf{x}_{C}, \mathbf{x}_{W}\right)
\end{aligned}
$$


The nonlinear functions $f_{W}, f_{C}: \mathbf{I} \subset \mathfrak{R}^{D} \rightarrow \mathbf{O} \subset \mathfrak{R}^{D}$ are continuous and provide the required adjustments to the states $\mathbf{x}_{W}$ and $\mathbf{x}_{C}$ respectively of the DCS neural network. Considering the DCS as a discrete-time dynamical system, the states of the DCS training algorithm can be represented in the following manner in a state-space representation form using Equations (3.16) and (3.17).

$$
\frac{\Delta \mathbf{X}}{\Delta t}=\left[\begin{array}{c}
\frac{\Delta \mathbf{x}_{W}}{\Delta t} \\
\frac{\Delta \mathbf{x}_{C}}{\Delta t}
\end{array}\right]=\left[\begin{array}{l}
f_{W}\left(\mathbf{x}_{W}, \mathbf{x}_{C}\right) \\
f_{C}\left(\mathbf{x}_{C}, \mathbf{x}_{W}\right)
\end{array}\right]
$$

\subsection{Lyapunov Stability Analysis of the DCS}

The goal of adaptive vector quantization is to move the weight vectors associated with the neural network in a manner that preserves the topology of the input data manifold. An ideal adaptive vector quantization algorithm would move the weight vectors asymptotically closer to the presented data. Therefore, it is of importance here to investigate if the weight vectors of the DCS neural network converge to an equilibrium point in reality. The question then is:

- By how much can the evolving state of a DCS learning deviate from a previously learned state?

Since the DCS represents a time-varying learning paradigm, where the state of learning evolves according to the difference relations of Equation (3.18), a Lyapunov theory based stability analysis is presented here as an abstract answer to the question.

Lyapunov's direct method (also known as the Lyapunov's second method), in particular, can be easily and systematically applied to validate the existence of stable states of nonlinear dynamical systems $[57,59,61,65]$. In order to claim that the DCS neural network is stabilizing during its adaptation from any data configuration of a stationary data manifold, it is required (though not necessary) to show that there exists a valid Lyapunov function for the 
DCS that is decreasing monotonically over time on successive applications of the neural network training algorithm.

The goal of the DCS is to overlay neurons (using weight vectors) over the presented data manifold in a topology-preserving manner such that nearby data patterns are mapped using neighboring neurons of the DCS network $[17,18,37]$. In order to generate a topologypreserving feature map of the presented data manifold, $M \subset \mathbf{I} \subset \mathfrak{R}^{D}$, the DCS network is initialized with two neurons that are connected to each other with connection strength of value 1. If it is determined that the error in the DCS network falls below a predefined threshold, additional neurons are introduced into the network. This process is repeated until the map generated by the DCS reaches a pre-specified degree of accuracy in its representation of the presented data $[17,18]$.

The addition of new neurons into the DCS network is based on resource values, a local error information associated with every DCS neuron. In most cases, the Euclidean distance between the data patterns of the training data manifold, $\mathbf{m} \in M \subset \mathbf{I} \subset \mathfrak{R}^{D}$ and the positions of the best matching units (BMUs), $\mathbf{w}_{b m u} \in \mathbf{W}_{D \times N} \subset \mathbf{O} \subset \mathfrak{R}^{D}$ serves as a measure for resource. Since resource is a measure of the local error information associated with each neuron, an average resource value can serve the purpose of a Lyapunov function for the DCS neural network. While considering the DCS adaptation as a discrete-time dynamical system, the end of a learning cycle followed by the addition of a new neuron can be treated as a time step. A Lyapunov function for the DCS can then be formulated in the following manner.

$$
V=\frac{1}{N} \sum_{\forall \mathbf{m} \in \mathbf{M}}\left\|\mathbf{m}-\mathbf{w}_{b m u(\mathbf{m})}\right\|
$$

The Lyapunov function that is given in Equation (3.19) is commonly known in the neural network community as the quantization error. The constructed Lyapunov function (Equation (3.19)) is a measure of the average resource value, the error associated with the DCS neural network representation of the input data manifold. In essence, the Lyapunov 
function is a measure the amount of topology of the input data manifold that is being preserved by the map generated by the neural network.

It is of interest here to show that the DCS neural network is stable when learning from a stationary (or non-changing) data manifold. It should be realized that even for the case of the neural network learning from a single stationary data manifold, there are infinite possible configurations of data, and it is not practical to provide heuristic evidence to support the claim that the DCS learning is stable for all possible configurations of stationary data. An analytical result showing that the Lyapunov function is decreasing over time implies that the neural network is stabilizing during its adaptation from a stationary data manifold. The following mathematical derivation is presented here as an analytical stability result that guarantees a stabilizing adaptation in the DCS neural network. The analytical result is applicable for the case where the DCS learning is based from a stationary (or non-changing) data manifold, $\mathbf{M} \in \mathbf{I} \subset \mathfrak{R}^{D}$.

Theorem 3.1 Stability of the DCS

Let $V(G, t): O \subset \mathfrak{R}^{D} \rightarrow \mathfrak{R}$, be a scalar function constructed for the map $G\left(M, W_{D \times N}, C_{N \times N}\right): I \subset \mathfrak{R}^{D} \rightarrow O \subset \mathfrak{R}^{D}$ generated by the online learning neural network from an input manifold $M \in I \subset \mathfrak{R}^{D}$. If $M$ remains fixed, then for any $\varepsilon>0$ we can find an integer $\delta>0$ such that for all $t>\delta$ we have $V(G, t)<\delta$.

Proof A detailed mathematical proof is provided in the Appendix

In order to exemplify the stability properties of adaptation in the DCS in terms of a monotonically decreasing Lyapunov function, consider the following example of the DCS learning from a 2-dimensional stationary data manifold $\mathbf{M} \subset \mathbf{I} \subset \mathfrak{R}^{2}$. The stationary data manifold chosen in this case is a twin-spiral function. A twin-spiral function represents a classical data-clustering problem that needs to be solved by overlaying neurons over the functions such that only neurons from the same spiral become connected. 
The twin-spiral data for this experiment is obtained from the Carnegie Mellon University's archive for artificial neural network benchmark data [66, 67]. The density of the twin-spiral function is set to a value of 5 and the spiral-radius is set to 6.5 . The program twospirals.c [67] generates 962 elements of twin-spiral data in a two-dimensional plain. Figure 3.1 shows a snapshot of the DCS when the neural network is initiated with two nodes that are connected to each other with connection strength of value 1 . The neural network training data, the twin-spiral function is shown in the background of Figure 3.1. Figure 3.2 shows the Lyapunov function (Equation (3.19)) of the initial state of the DCS. The initial value of the Lyapunov function of the DCS is 2764 (Figure 3.2).

Since the current analysis concerns neural network learning from a fixed (or nonchanging) data manifold, the introduction of an additional node into the network represents the completion of a learning cycle. Figure 3.3 shows a snapshot of the DCS neural network after 50 learning cycles. The neural network training data, the twin-spiral function is shown in the background of Figure 3.3. Figure 3.4 shows the Lyapunov function (Equation (3.19)) of the corresponding state of the DCS. Figure 3.5 shows a snapshot of the DCS neural network after 100 learning cycles. The neural network training data, the twin-spiral function is shown in the background of Figure 3.5. Figure 3.6 shows the Lyapunov function (Equation (3.19)) of the corresponding state of the DCS. Figure 3.7 shows a snapshot of the DCS neural network after 150 learning cycles. The neural network training data, the twin-spiral function is shown in the background of Figure 3.7. Figure 3.8 shows the Lyapunov function (Equation (3.19)) of the corresponding state of the DCS. Figure 3.9 shows a snapshot of the DCS neural network after 200 learning cycles. The neural network training data, the twin-spiral function is shown in the background of Figure 3.9. Figure 3.10 shows the Lyapunov function (Equation (3.19)) of the corresponding state of the DCS. It can be observed from the figures that as the number of nodes in the DCS is increased, the neural network generates efficient (topology-preserving) representations of the given training data, which in this case, is the twin-spiral function. The improvement in the efficiency of topology preservation by the DCS neural network is captured and indicated by the monotonically decreasing behavior of the Lyapunov function. 


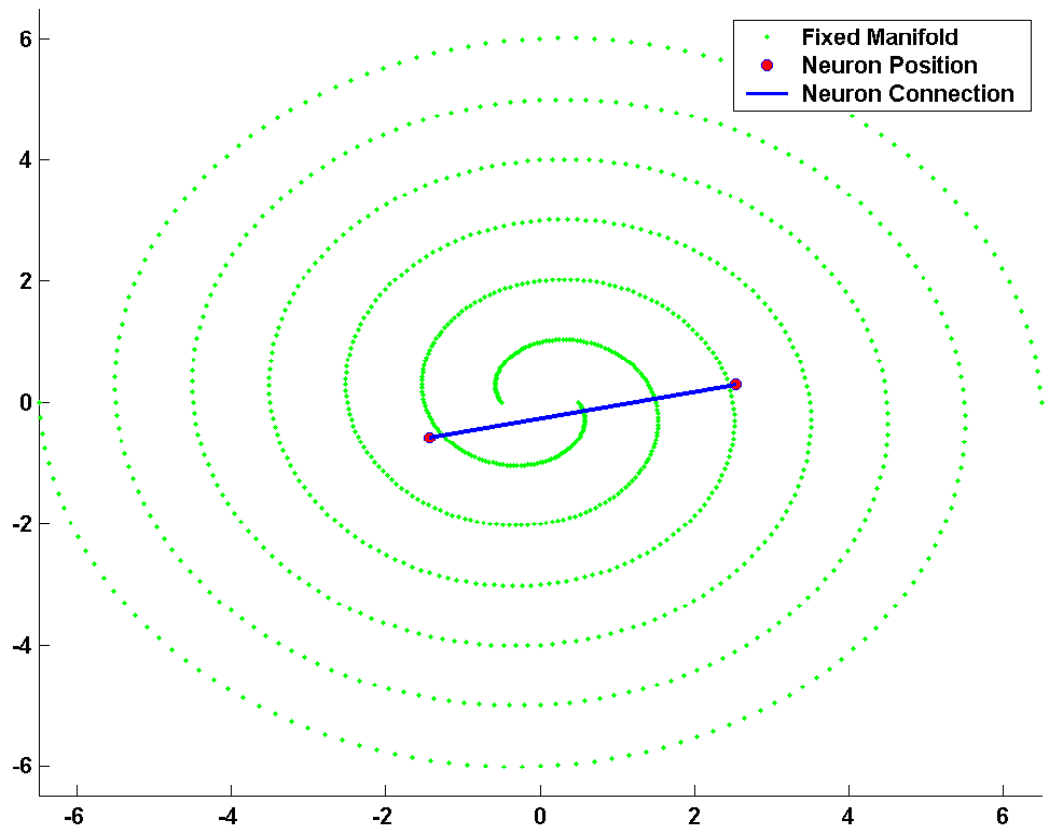

Figure 3.1 Snapshot of the DCS NN initiation with two connected nodes

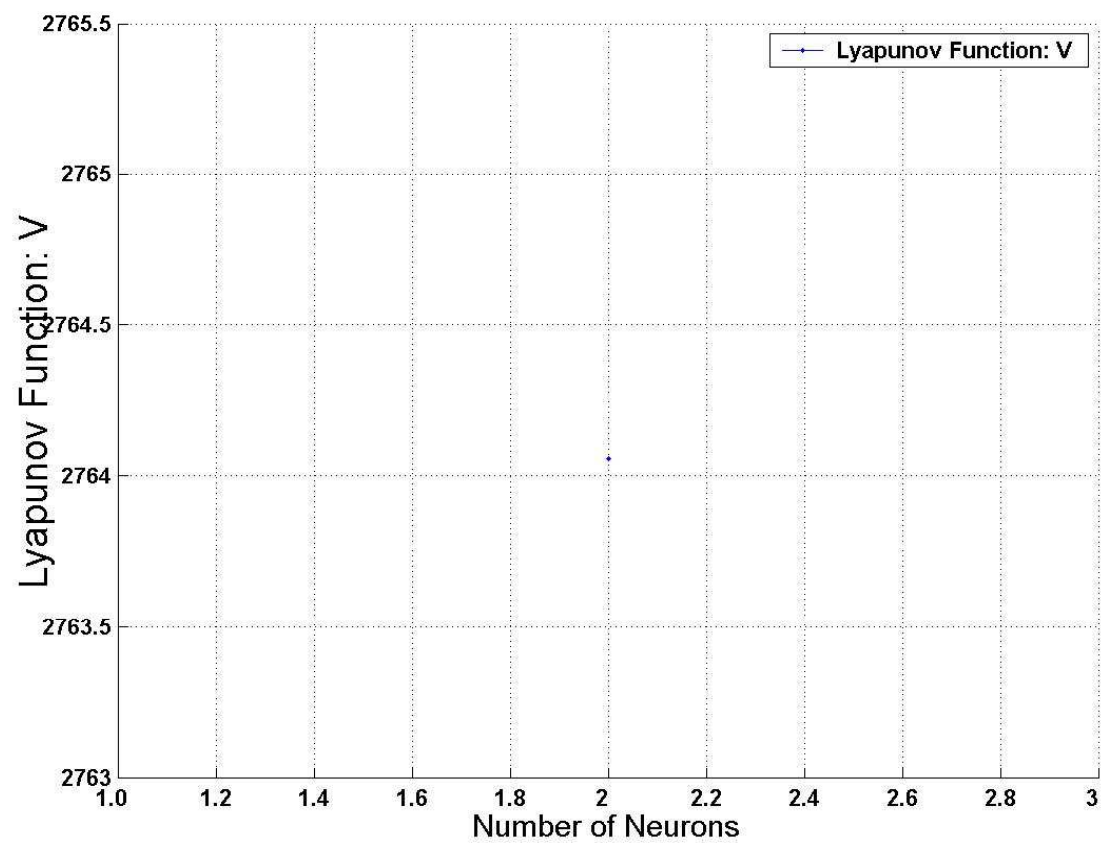

Figure 3.2 Lyapunov function of the DCS: initial value is $\mathbf{2 7 6 4}$ 


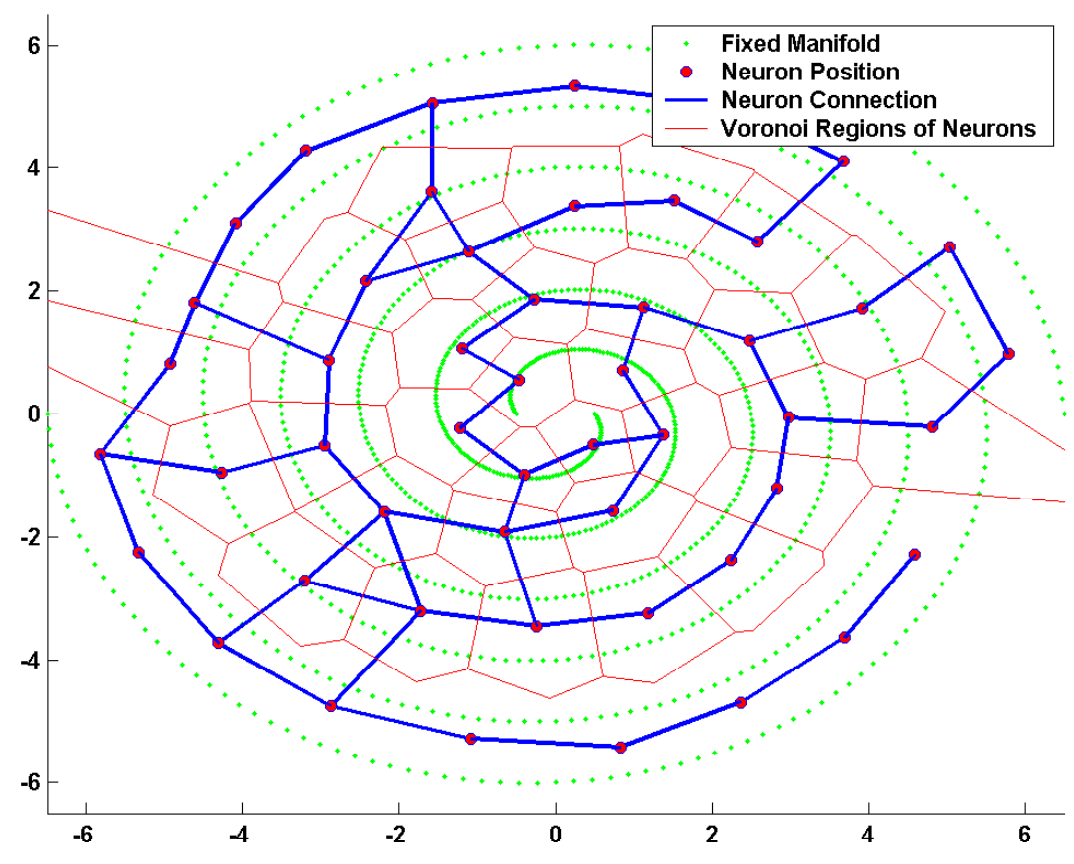

Figure 3.3 Snapshot of the DCS after 50 learning cycles

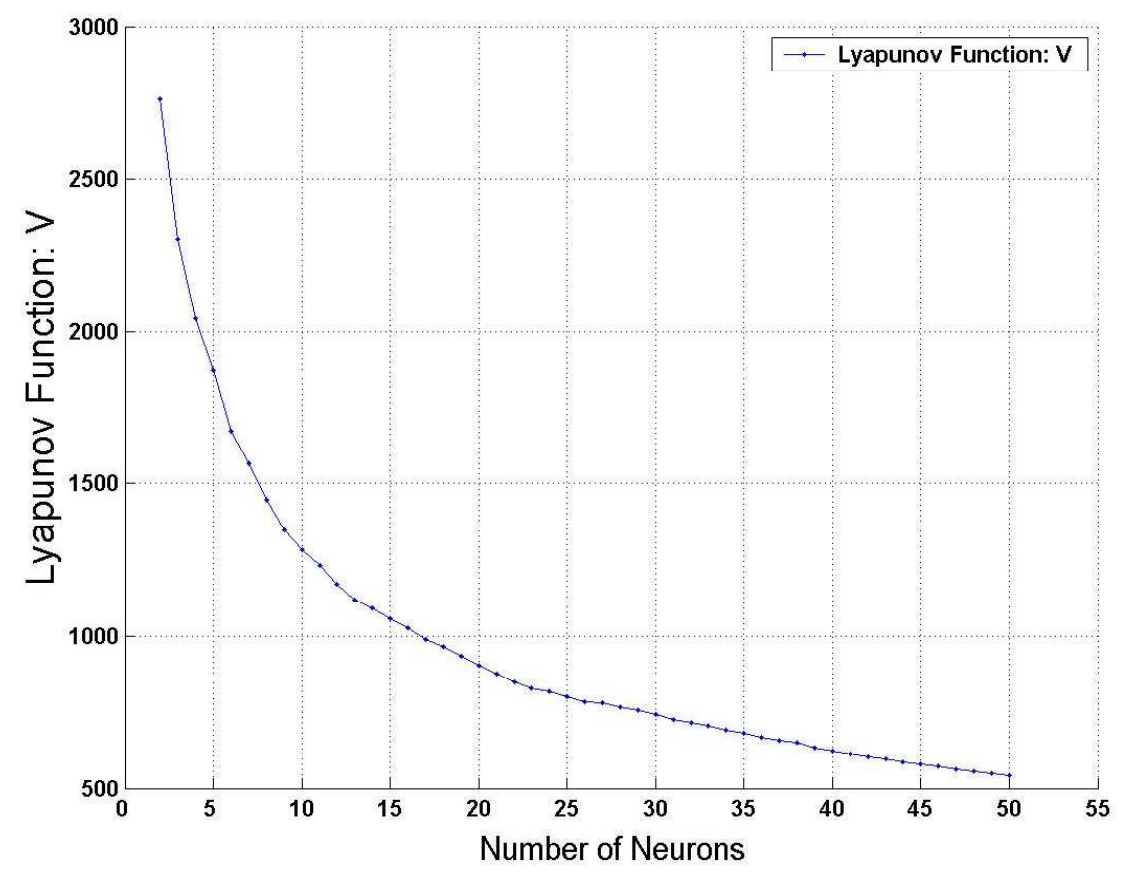

Figure 3.4 Lyapunov function of the DCS after 50 learning cycles 


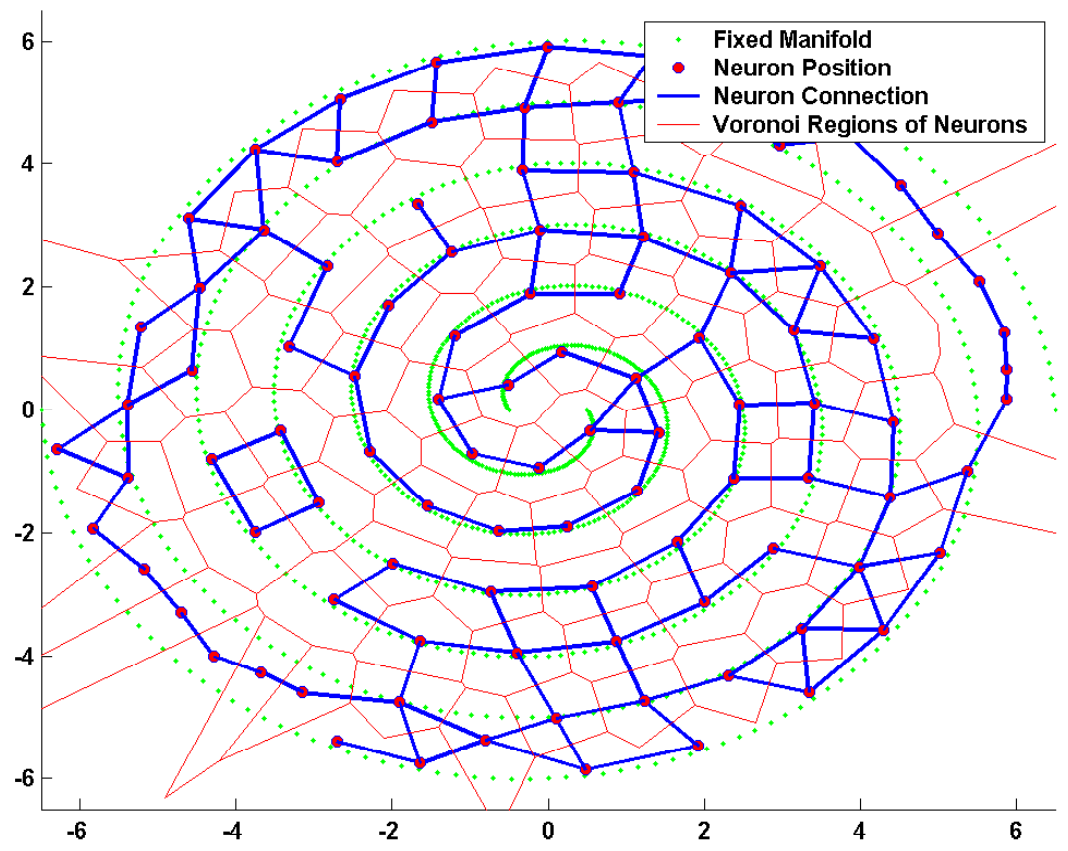

Figure 3.5 Snapshot of the DCS after 100 learning cycles

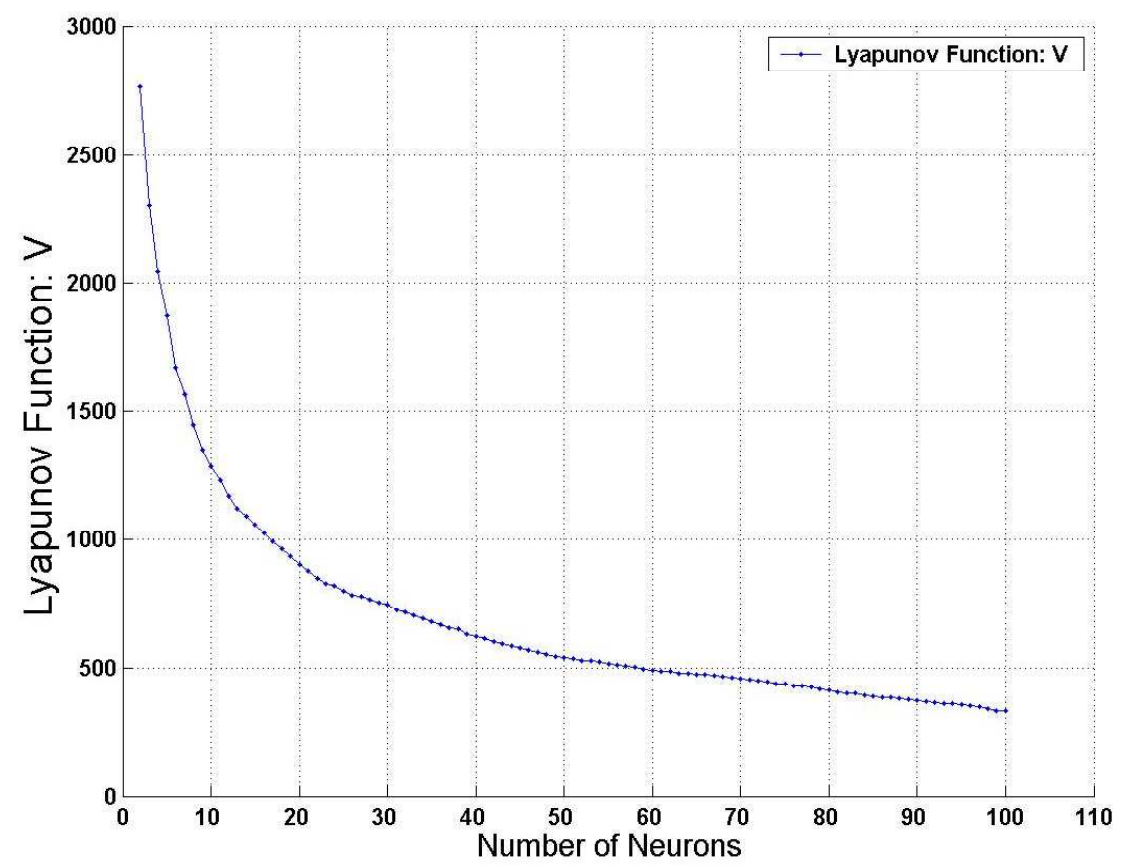

Figure 3.6 Lyapunov function of the DCS after 100 learning cycles 

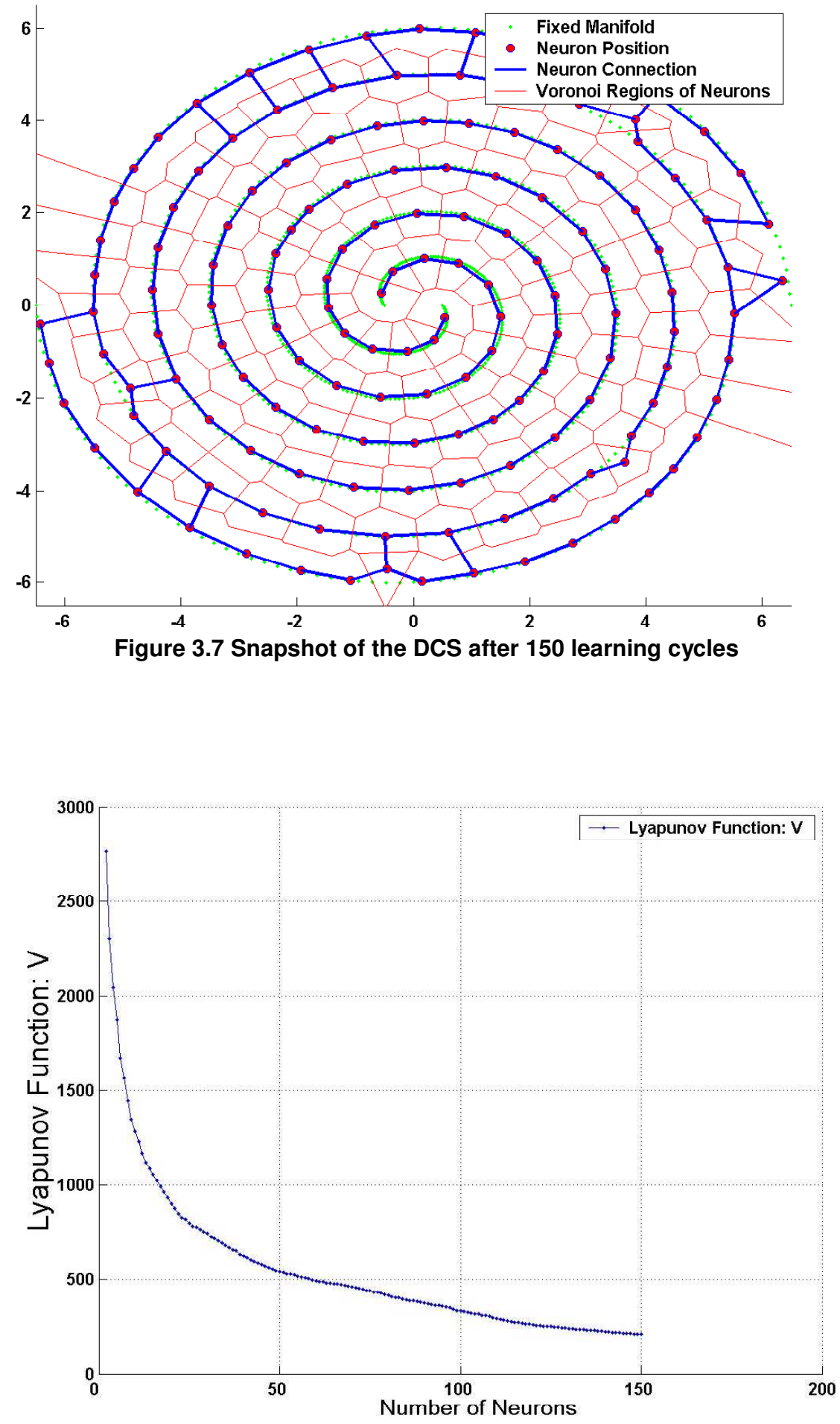

Figure 3.8 Lyapunov function of the DCS after 150 learning cycles 


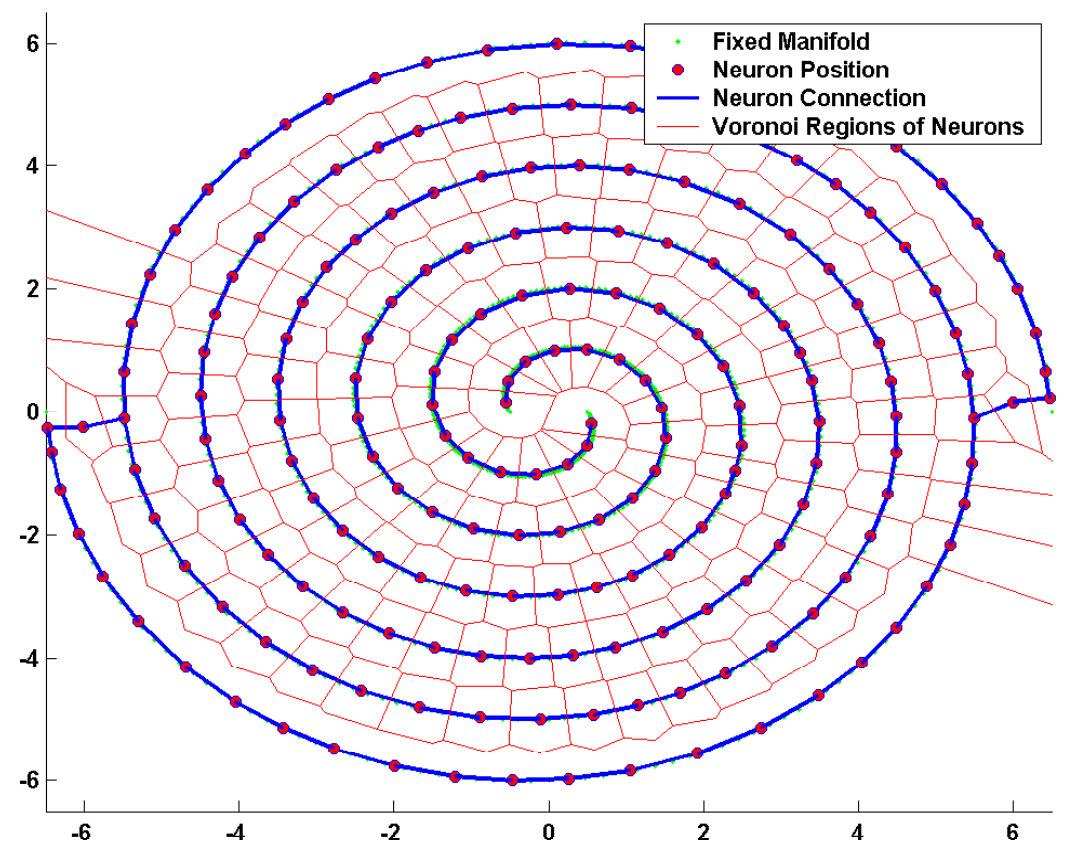

Figure 3.9 Snapshot of the DCS after 200 learning cycles

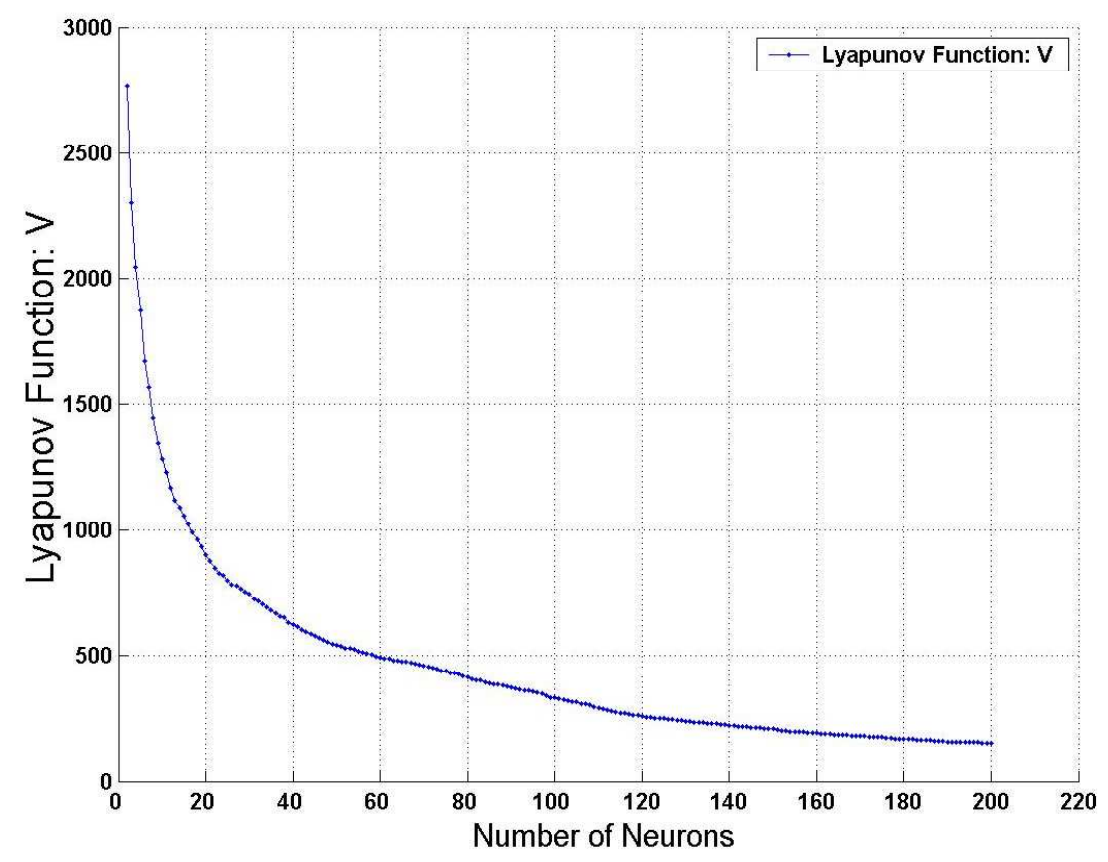

Figure 3.10 Laypunov function of the DCS after 200 learning cycles 
The analytical and experimental results presented here demonstrate that the constructed Lyapunov function (Equation (3.19)) is decreasing monotonically over some learning cycles when the DCS neural network is presented with a stationary data set. In other words, the heuristic results presented here support the mathematical derivation in that claims that the DCS neural network is stable, when the learning is based from a stationary (nonchanging) training data. 


\section{Chapter}

\section{Online Stability Monitoring}

Stability and convergence of online adaptation were proposed for consideration as heuristic measures of correctness for the sake of system safety in Chapter 1. Some results relative to the theoretical analysis of the stability properties of online adaptation in neural networks were discussed in Chapter 3 . It is crucial to realize that the stability results presented in Chapter 3 were based on the adaptation of neural networks from stationary of fixed data manifolds. Learning and adaptation from a stationary configuration of data implies that once the learning algorithm is presented with a certain data, the configuration of data remains unchanged throughout the learning process. In other words, the delineated stability boundaries of online adaptation in neural networks that were presented in Chapter 3 can guarantee a stabilizing neural network adaptation only when the learning is based from a stationary or fixed data manifold.

The IFCS system has the ability to function under a failed flight condition - for example, when the aircraft's stabilator or aileron becomes locked or damaged during flight $[69,72]$. It is experimentally observed in this research that under such off-nominal operating conditions, the adaptation in the embedded online learning component may not necessarily be based on stationary representatives of data but rather on evolving or varying data manifolds. In such cases, the analytical results presented Chapter 3 cannot be used for ensuring a stable online adaptation. If the embedded online learning component tends to become unstable, then it may cause the overlaid adaptive system to lose its functionality, eventually leading to a catastrophic system failure. It is therefore necessary to ensure that the embedded online learning component is stable even when the adaptation is based on varying representatives of data.

Ensuring guaranteed stability in online learning for all data domains, i.e. stationary and varying is not a possibility. This is because the non-deterministic nature of adaptive 
systems coupled with the stochastic nature of online learning prohibits distinguishing stable from unstable learning beforehand for all domains of adaptive system data. As the last resort for system safety, one needs to identify and detect unstable learning behavior. The real analysis challenge, therefore, is to be able to develop a procedure for monitoring online learning in order to detect unstable (or abnormal) learning behavior during the operation of the adaptive system under failure scenarios. Chapter 3 established that the stability of adaptation is an important consideration when working with online learning components in safety-critical systems. In the absence of an analytical stability proof, the following are the goals that must be achieved by a stability-monitoring scheme for online adaptation.

During the operation of the adaptive system under rapidly changing environmental conditions:

- Does the online learning tend to become unstable?

- Is it possible to detect the unstable learning conditions?

After experiencing unstable learning conditions:

- Does the online learning converge back to a stable state?

- Is it possible to realize when the learner converges back to a stable state?

Due to model-uncertainty associated with online learning within an adaptive system, adumbrating stability boundaries and detecting learning states in online learning that bifurcate from a stable behavior beforehand, for all operational conditions of the adaptive system, may not be a possibility. Therefore, no analysis technique can possibly provide precise answers to the questions posed earlier. Based on experimental evaluation of the stability properties of online learning behavior, it is proposed here that developing certain Lyapunov-like functions to monitor and detect unstable conditions in online learning can resolve this quandary.

In the context of the analysis of adaptation capabilities of the online learning within the IFCS system, the goal is to find an effective tool for monitoring and detecting unstable learning conditions. This research therefore proposes an online stability monitoring system that can detect unstable states in learning that tend to bifurcate away from a stable behavior. The 
online stability monitoring is designed to assess with the overall stability behavior of online learning during the operation of adaptive system under changing environmental conditions, i.e. when the analytical stability proofs of Chapter 3 fail to apply.

\subsection{Mathematical Formulation of Stability Monitors}

In the IFCS system, the online learning consists of several aspects of knowledge acquisition such as the best matching unit (bmu) adaptations, second best unit (sbu) adaptations, neighborhood ( $n b r)$ adaptations, and non-neighborhood (non-nbr) adaptations. The reader is referred to Section 2.2 in Chapter 2 for additional details on the individual learning aspects of online learning. This research proposes the development of an online stability monitoring system that is based on the construction of a set of Lyapunov-like functions. The idea here is that different Lyapunov-like functions analyze unstable learning for different aspects of online learning. Based on the research conducted at the laboratory, four stability monitors are proposed in an effort to diagnose unstable online learning behavior for conditions where the analytical stability proof of Chapter 3 fails to apply. A fundamental understanding of the various aspects of online learning is provided in Section 2.2 in Chapter 2. The four stability monitors proposed here are based on the following mathematical formulations.

Let $M \subset \Re^{D}$ be the given neural network training data manifold. For an input element of the training data manifold, $m \in M \subset \mathfrak{R}^{D}$, let $W_{b m u(m)}$ and $W_{s b u(m)}$ represent the weight centers (location in the Cartesian plane) of the best matching unit $(b m u)$ and the second best unit (sbu) respectively. For an input element, $m \in M \subset \mathfrak{R}^{D}$, let $W_{\{n b r(m)\}}$ represent the weight centers of the set of neighboring nodes that are connected to the $b m u$ of the input element. Similarly, let $W_{\{n o n-n b r(m)\}}$ represent the weight centers of the set of nonneighboring nodes that are not connected to the bmu of the input element.

\section{Definition 4-1 Stability monitor 1}


Stability monitor 1 is the cumulative Euclidean distance between each data pattern of the given training data manifold, $m \in M \subset \mathfrak{R}^{D}$ and the corresponding best matching units.

$$
\text { Monitor } 1=\sum_{\mathbf{m} \in \mathbf{M}}\left\|\mathbf{m}-\mathbf{w}_{b m u(\mathbf{m})}\right\|
$$

\section{Definition 4-2 Stability monitor 2}

Stability monitor 2 is the cumulative Euclidean distance between each data pattern of the training data manifold, $m \in M \subset \mathfrak{R}^{D}$ and the second best units.

$$
\text { Monitor } 2=\sum_{\mathbf{m} \in \mathbf{M}}\left\|\mathbf{m}-\mathbf{w}_{s b u(\mathbf{m})}\right\|
$$

\section{Definition 4-3 Stability monitor 3}

Stability monitor 3 is the cumulative average Euclidean distance between each data pattern of the training data manifold, $m \in M \subset \mathfrak{R}^{D}$ and the neighboring nodes that are connected to the corresponding best matching units.

$$
\text { Monitor } 3=\sum_{\mathbf{m} \in \mathbf{M}} \underset{j \in\{n b r(b m u)\}}{\operatorname{avg}}\left\{\left\|\mathbf{m}-\mathbf{w}_{j}\right\|\right\}
$$

\section{Definition 4-4 Stability monitor 4}

Stability monitor 4 is the cumulative average Euclidean distance between each data pattern of the training data manifold, $m \in M \subset \mathfrak{R}^{D}$ and the non-neighboring nodes that are not connected to the corresponding best matching units.

$$
\text { Monitor } 4=\sum_{\mathbf{m} \in \mathbf{M}} \underset{j \notin\{n b r(b m u)\}}{\operatorname{avg}}\left\{\left\|\mathbf{m}-\mathbf{w}_{j}\right\|\right\}
$$

The stability monitors 1 through 4 (Equations (4.20) through (4.23)) essentially measure the accuracy of the map generated by the online learning neural network in terms of the amount of topology of the input data that is being preserved. An online stability monitoring system that is composed of the Lyapunov-like functions such as the ones given in Equations (4.20) through (4.23) can provide an insight into the understanding of the stability properties of online learning. 


\subsection{Multiple-Monitor Based Approach - Why?}

The discussion in Section 2.2 indicates that online learning in neural networks is comprised of several aspects of knowledge acquisition including the bmu, sbu, nbr, and nonnbr adaptations. It is observed in this research that due to the stochastic nature of online learning, it is not possible to capture stability-information from all aspects of online learning using a single stability monitor. In other words, no single stability monitor was able to completely detect unstable learning for all of the tested operational conditions of the adaptive system. This is the motivation behind proposing a multiple-monitoring based approach for detecting unstable online learning conditions. This concept becomes evident when the online learning component, in our case the online learning DCS neural network, encounters rapidly varying, divergent data manifolds. As an example, consider a typical situation of a failed flight control condition - an aircraft's left stabilator becomes locked during flight at an angle of $+3^{\circ}$. A stabilator is a part of the modified control surfaces of the F15B fighter aircraft shown in Figure 1.6. The online learning DCS algorithm is presented with 200 vectors of training data from 10 seconds of flight corresponding to a sampling frequency of $20 \mathrm{~Hz}$.

In our case of online learning, a data vector is a 6-dimensional vector of data consisting of 4 independent aircraft parameters and 2 dependant, control derivative error variables. The aircraft parameters, the Mach number, altitude, $\alpha$ (angle of attack), and $\beta$ (sideslip angle) represent the 4 independent variables. The 2 control derivative error variables are the partial derivative of the normal force with respect to the angle of attack ( $\Delta C z \alpha l$ ), and the partial derivative of the normal forces with respect to the stabilator deflection ( $\Delta C z \alpha s)$. The two control derivative errors are measured in $\mathrm{deg}^{-1}$.

The online learning DCS neural network was trained using the data collected from the high fidelity NASA-WVU adaptive flight simulator [42, 43, 45]. The user interface of this simulator is shown in Figure 4.1. The simulator corresponds to the architecture and the functionality of the IFCS system that is shown in Figure 1.5 in Chapter 1. The 6-dimensional, 
200 data vectors are generated by the adaptive flight simulator during the transition of the aircraft from nominal (no-failure) to a failed flight-condition.

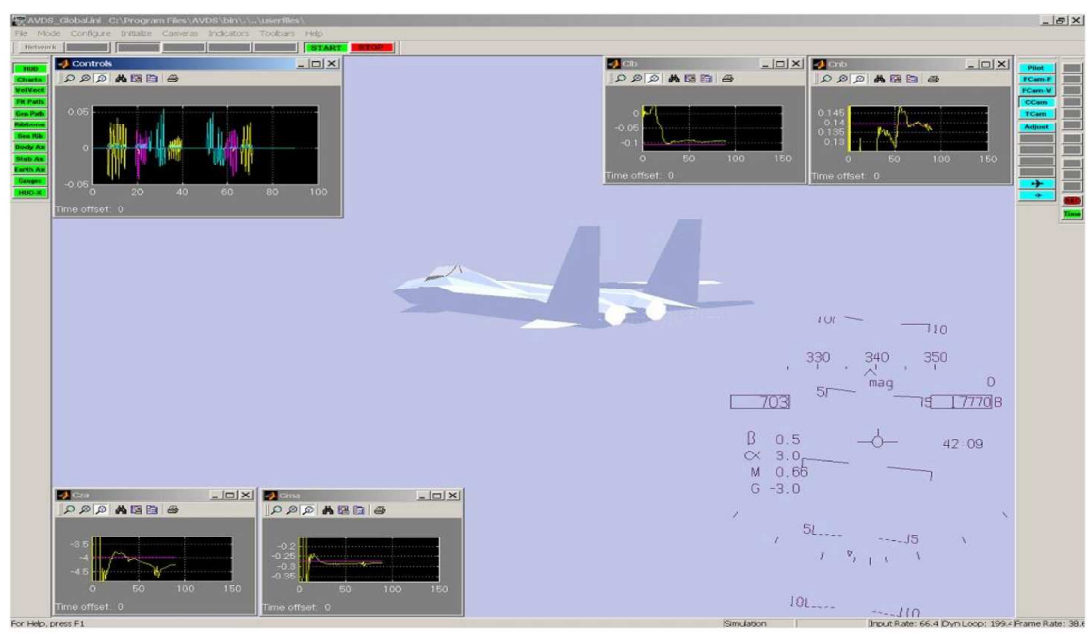

Figure 4.1 User interface of NASA-WVU adaptive flight simulator

The data from the simulator is sampled with a frequency of $20 \mathrm{~Hz}$ that corresponds to 20 cycles of 6-dimensional online data generated in real-time from the adaptive flight simulator for a second of flight-operation. In our case, it is chosen to present the online learning neural network with cycles of data in an online manner so that each cycle consists of a single data vector. This means that in our case of online learning, 200 cycles of online data generated at the rate of $20 \mathrm{~Hz}$ of sampling corresponds to 10 seconds of flight. The first 99 cycles represent the data generated by the adaptive flight simulator under nominal or no-failure conditions. The $100^{\text {th }}$ cycle of data represents the data generated by the adaptive flight simulator as the aircraft-fault has been injected. Therefore, around the 100th cycle of data, the environment in the adaptive system degrades from nominal to a stressed mode of operation. Because the online learning DCS neural network is embedded into the flight control system, when the DCS encounters inconsistent data representations (around $100^{\text {th }}$ cycle) it will likely deviate from stable learning state. The goal of the online stability monitoring system is to numerically capture deviations in stable learning states of the online learning neural network. 
The stability monitoring system proposed here comprises of four monitors (Equations (4.20) through (4.23)) that have been specifically developed to detect unstable learning conditions from different aspects of online learning. A spike (abrupt increase) in the values of any or all-4 monitors indicates unstable online adaptation conditions in the neural network. Since there may be noise associated with the monitor values (either due to noise in the neural network input or otherwise) that could raise a false alarm, a spike in the monitor value is deemed predominant if it is above a certain threshold value. For this case of online learning, the threshold is set to value of $10 \sigma$, where the term $\sigma$ represents the standard deviation of the initial values of the monitor. The initial value of the monitor is referred here as the baseline value. For the current analysis, the first 20 monitor values (corresponding to the first 20 cycles of flight data) are collectively considered as the monitor baseline. The monitor baseline can be thought of as the startup values before the detection phase begins. Note that the value of the threshold gain is set to a value of 10 in order to reduce false alarms, and increase the probability of detection. The gain value that is specified here is based on observations from several runs of stability monitoring experiments that were conducted in the lab.

The following observations were made for the failed flight control condition - aircraft's left stabilator becomes locked during flight at an angle of $+3^{\circ}$. Figure 4.2 shows the stability monitor 1 (Equation (4.20)), after presenting the online learning neural network with the data generated under failed flight-conditions. The stability monitor 1 (Figure 4.2) shows no predominant spikes (no values are above the $10 \sigma$ threshold). In other words, stability monitor 1 , in this case, was unable to detect any unstable online adaptation. Figure 4.3 shows stability monitor 2 (Equation (4.21)), after presenting the online learning neural network with the data generated under failed flight-conditions. The stability monitor 2 (Figure 4.3) shows no predominant spikes (no values are above the $10 \sigma$ threshold). It is deemed that the stability monitor 2, in this case, is also unable to detect unstable online adaptation. Figure 4.4 shows stability monitor 3 (Equation (4.22)), after presenting the online learning neural network with the data generated under failed flight-conditions. Unlike Stability monitors 1 and 2 (Figure 4.2 
and Figure 4.3), the stability monitor 3 in Figure 4.4, shows a predominant spike in its value $(151.95 \sigma)$ when the neural network is presented with the data generated under failed flight conditions (cycle 100). Figure 4.5 shows stability monitor 4 (Equation (4.23)), after presenting the online learning neural network with the data generated under failed flight-conditions. The stability monitor 4 in Figure 4.5 shows a predominant spike in its value $(106.83 \sigma)$ when the neural network is presented with the data generated under failed flight conditions (cycle 100). For this example of online learning from failed flight conditions, stability monitors 3 and 4 (Figure 4.4 and Figure 4.5) successfully detected unstable online adaptation. Stability monitors 1 and 2 (Figure 4.2 and Figure 4.3), in this case, were unable to detect unstable online adaptation.

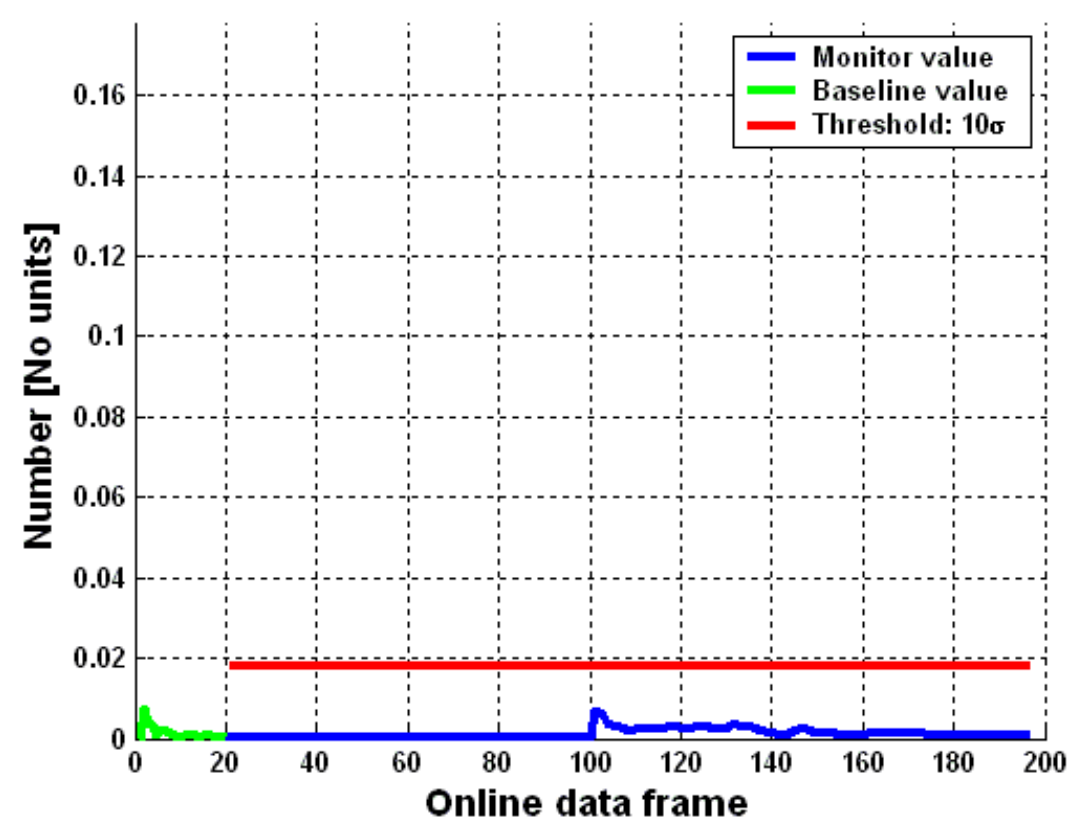

Figure 4.2 Online stability monitor 1 (Equation (4.20)) 


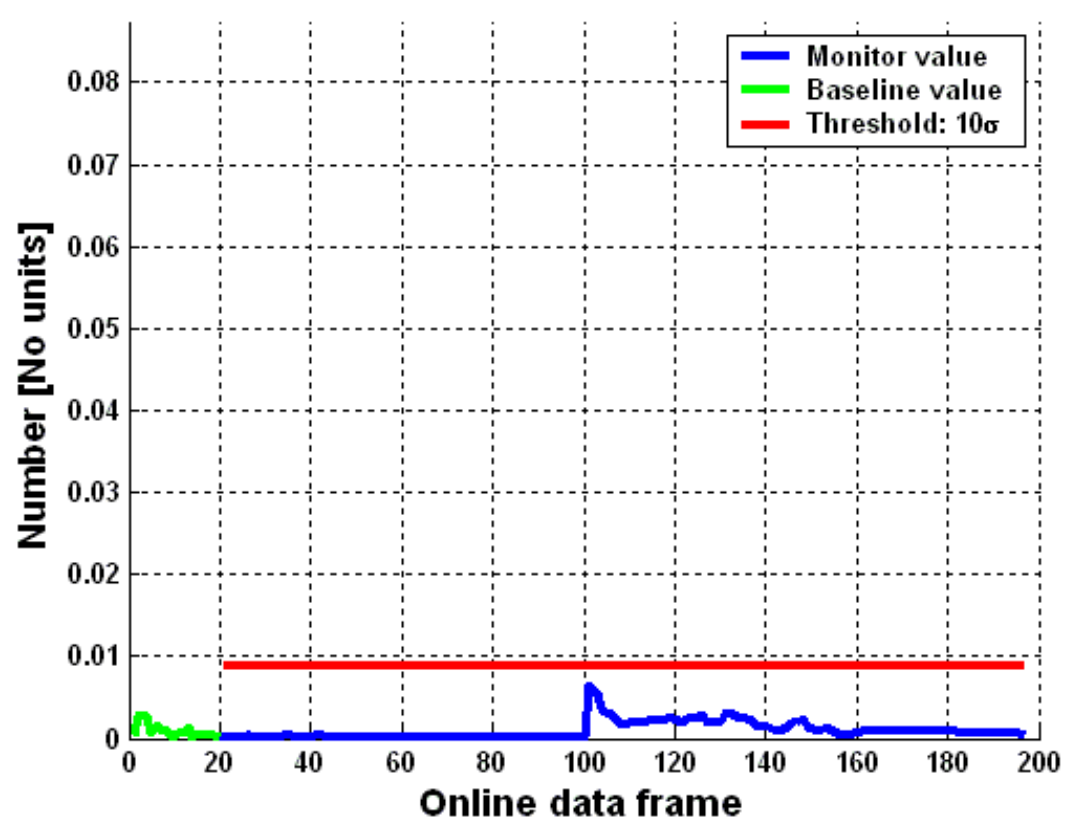

Figure 4.3 Online stability monitor 2 (Equation (4.21))

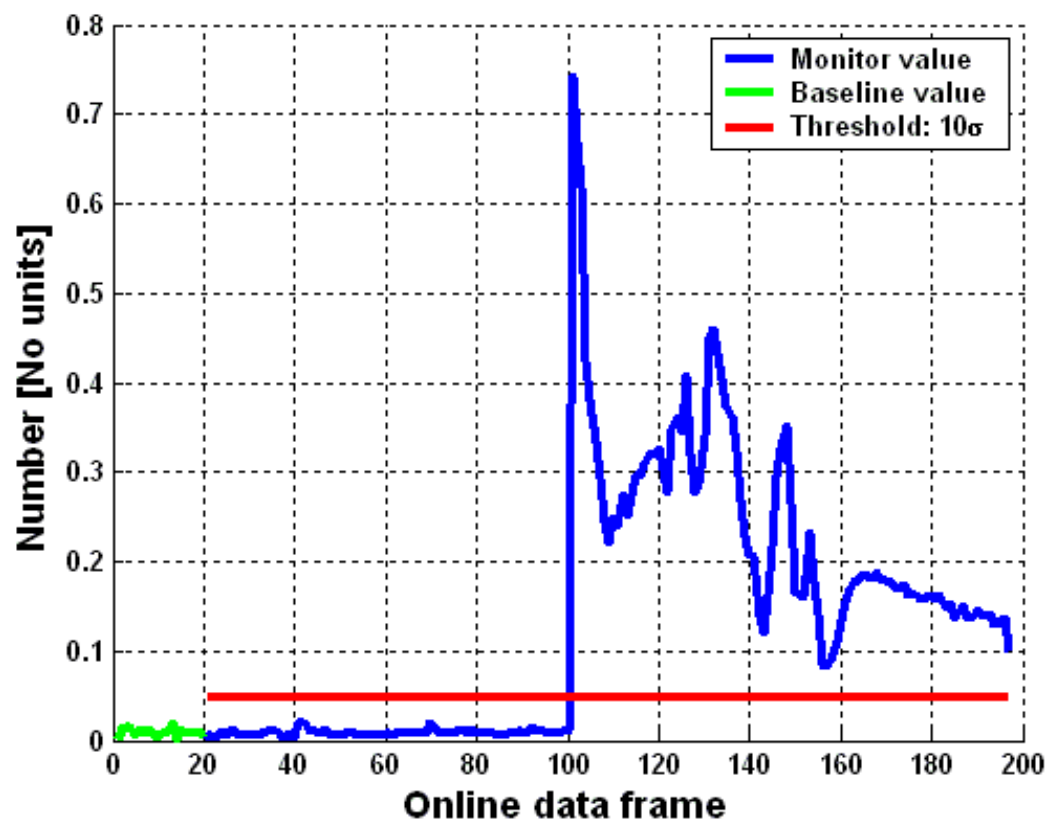

Figure 4.4 Online stability monitor 3 (Equation (4.22)) 


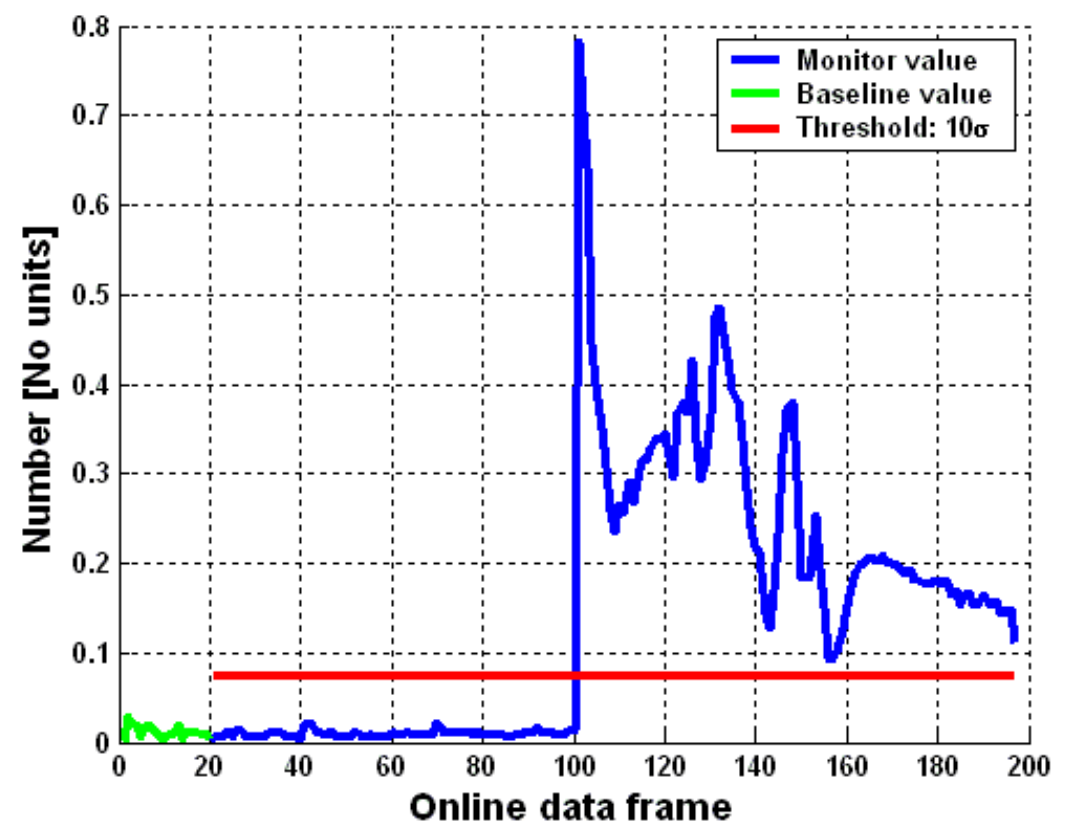

Figure 4.5 Online stability monitor 4 (Equation (4.23))

As the second example, consider another typical situation of a failed flight control surface - aircraft's left stabilator becomes missing by $50 \%$ during flight. A stabilator is a part of the modified control surfaces of the F15B fighter aircraft shown in Figure 1.6 in Chapter 1. The data from the simulator is sampled with a frequency of $20 \mathrm{~Hz}$ that corresponds to 20 cycles of 6-dimensional online data generated in real-time from the adaptive flight simulator for a second of flight-operation. As in the first example, the online learning DCS algorithm is presented with 200 vectors of training data from 10 seconds of flight corresponding to a sampling frequency of $20 \mathrm{~Hz}$.

The first 99 cycles represent the data generated by the adaptive flight simulator under nominal or no-failure conditions. The $100^{\text {th }}$ cycle of data represents the data generated by the adaptive flight simulator as the aircraft-fault has been injected. Therefore, around the $100^{\text {th }}$ cycle of data, the environment in the adaptive system degrades from nominal to a stressed mode of operation. Because the online learning DCS neural network is embedded into the flight control system, when the DCS encounters inconsistent data representations (around cycle 100) it will likely deviate from stable learning state. The goal of the online stability 
monitoring system is to numerically capture deviations in stable learning states of the online learning neural network.

A spike (abrupt increase) in the values of any or all-4 monitors indicate an unstable online adaptation in the neural network. As in the previous experiment, a spike in the value of the monitor that is above the $10 \sigma$ threshold is deemed as a predominant spike. The term $\sigma$ represents the standard deviation of the monitor baseline (first 20 monitor values). Figure 4.6 shows stability monitor 1 (Equation (4.20)), after presenting the online learning neural network with the data generated under failed flight-conditions. The stability monitor 1 in Figure 4.6 shows a predominant spike in its value $(124.84 \sigma)$ when the neural network is presented with the data generated under failed flight conditions (cycle 100). Figure 4.7 shows stability monitor 2 (Equation (4.21)), after presenting the online learning neural network with the data generated under failed flight-conditions. The stability monitor 2 in Figure 4.7 shows a predominant spike in its value $(101.08 \sigma)$ when the neural network is presented with the data generated under failed flight conditions (cycle 100).

Figure 4.8 shows stability monitor 3 (Equation (4.22)), after presenting the online learning neural network with the data generated under failed flight-conditions. The stability monitor 3 in Figure 4.8 shows no predominant spikes in its values (no values are above the $10 \sigma$ threshold). In the current case of online learning, the stability monitor 3 is unable to detect unstable online adaptation. Figure 4.9 shows stability monitor 4 (Equation (4.23)), after presenting the online learning neural network with the data generated under failed flightconditions. The stability monitor 4 in Figure 4.9 shows no predominant spikes in its values (no values are above the $10 \sigma$ threshold). In the current case of online learning, the stability monitor 4 is unable to detect unstable online adaptation. For this example of online learning from failed flight conditions, stability monitors 1 and 2 (Figure 4.6 and Figure 4.7) detected unstable online adaptation. However, stability monitors 3 and 4 (Figure 4.8 and Figure 4.9) were unable to detect unstable online adaptation. 


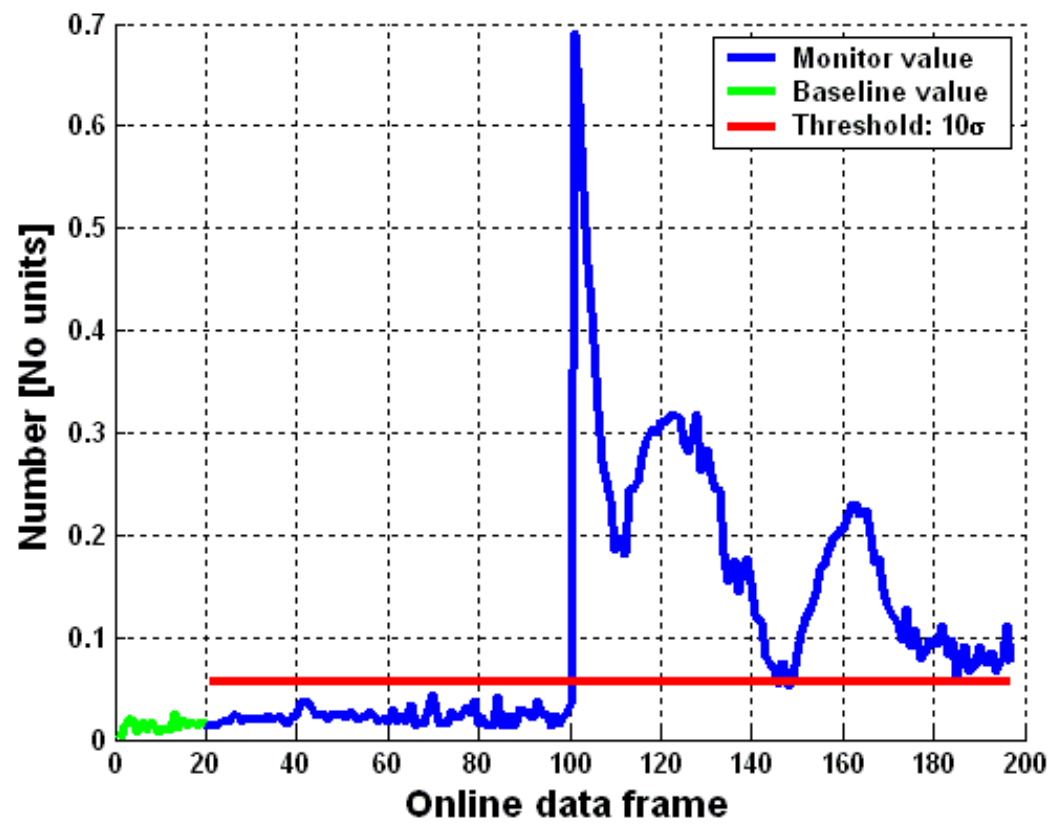

Figure 4.6 Online stability monitor 1 (Equation (4.20))

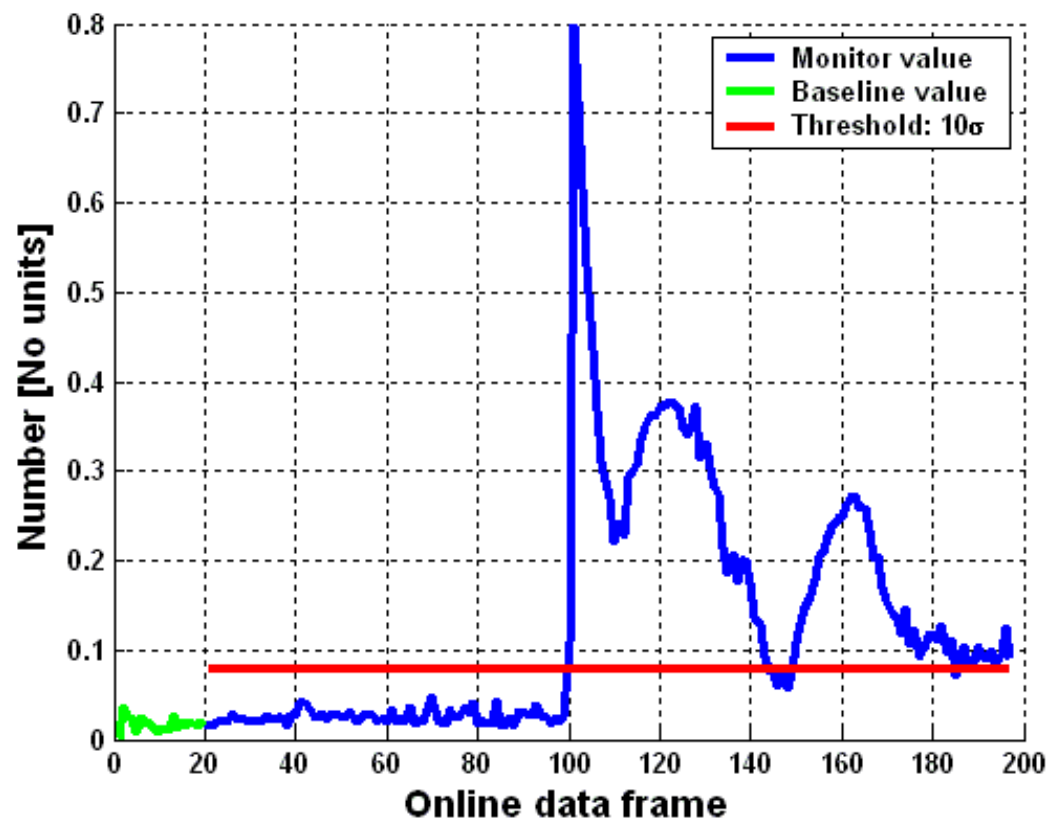

Figure 4.7 Online stability monitor 2 (Equation (4.21)) 


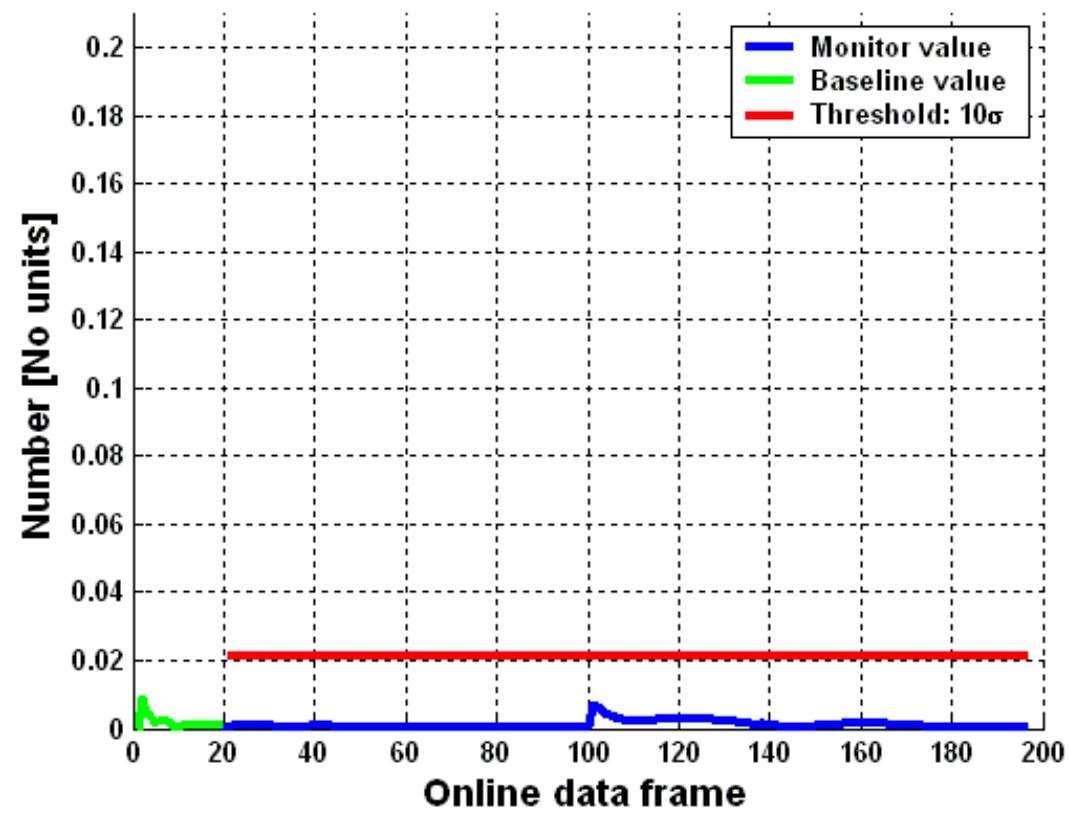

Figure 4.8 Online stability monitor 3 (Equation (4.22))

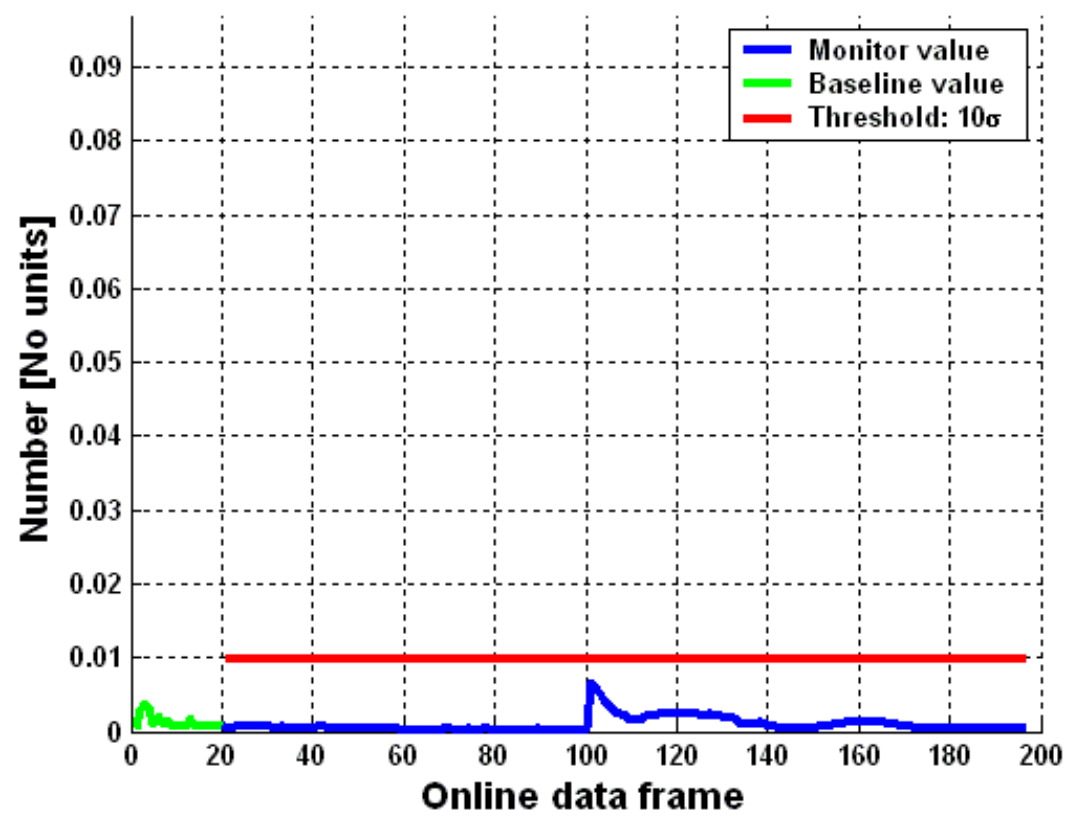

Figure 4.9 Online stability monitor 4 (Equation (4.23)) 
Finally, as the third example, consider a typical situation of a nominal (no-failure) flight condition. The data from the simulator is sampled with a frequency of $20 \mathrm{~Hz}$ that corresponds to 20 cycles of 6-dimensional online data generated in real-time from the adaptive flight simulator for a second of flight-operation. As in the first example, the online learning DCS algorithm is presented with 200 vectors of training data from 10 seconds of flight corresponding to a sampling frequency of $20 \mathrm{~Hz}$. Unlike the previous examples, all-200 cycles represent the data generated by the adaptive flight simulator under nominal or no-failure conditions. Because the online learning DCS neural network is embedded into the flight control system, the DCS should not encounter any inconsistent data representations and it should likely remain stable throughout learning. The goal of the online stability monitoring system is to numerically capture the stable learning behavior of the online learning neural network.

As in the previous experiments, a spike in the value of the monitor that is above $10 \sigma$ is deemed as a predominant spike. The term $\sigma$ represents the standard deviation of the monitor values until the current value. The stability monitor 1 (Equation (4.20)) that is shown in Figure 4.10 indicates no predominant spikes in its values (no values are above the $10 \sigma$ threshold). This indicates that the stability monitor 1 shows stable online adaptation conditions. The stability monitor 2 (Equation (4.21)) that is shown in Figure 4.11 indicates no predominant spikes in its values (no values are above the $10 \sigma$ threshold). This indicates that like the stability monitor 1 (Figure 4.10), the stability monitor 2 (Figure 4.11) also shows stable online adaptation conditions. The stability monitor 3 (Equation (4.22)) that is shown in Figure 4.12 indicates no predominant spikes in its values (no values are above the $10 \sigma$ threshold). This indicates that the stability monitor 3 also shows stable online adaptation conditions. The stability monitor 4 (Equation (4.23)) that is shown in Figure 4.13 indicates no predominant spikes in its values (no values are above the $10 \sigma$ threshold). This indicates that the stability monitor 4 also shows stable online adaptation conditions. 


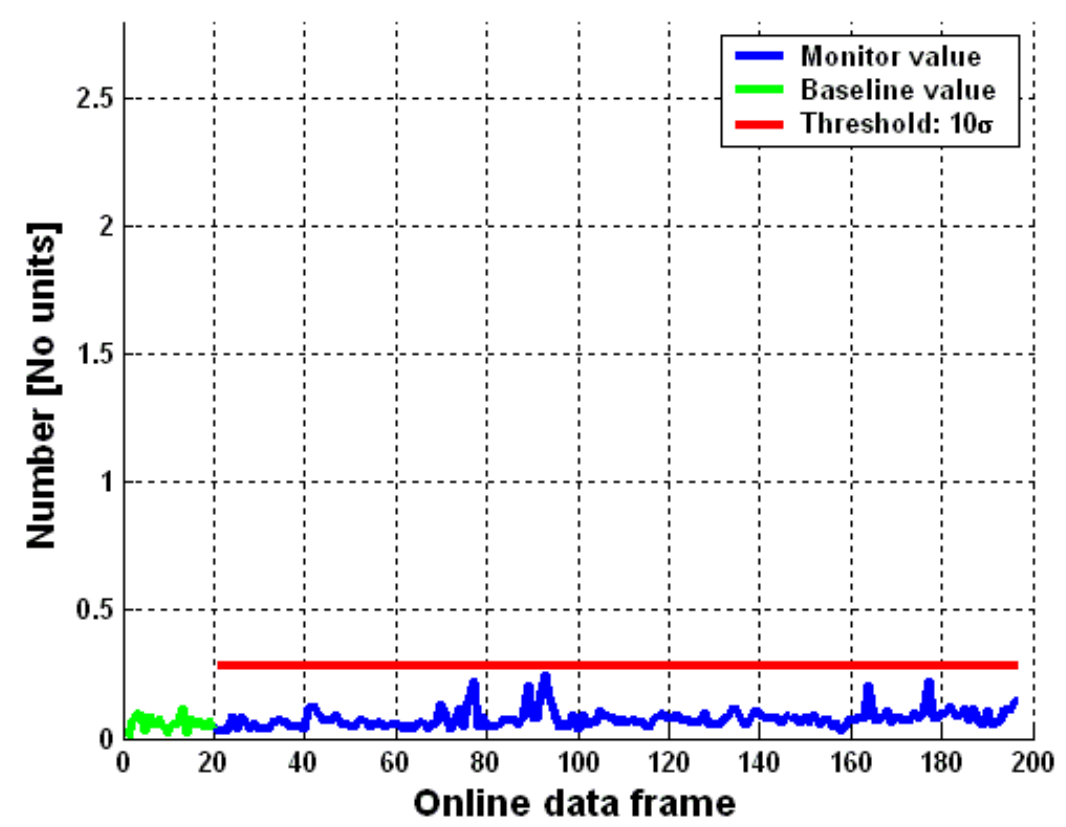

Figure 4.10 Online stability monitor 1 (Equation (4.20))

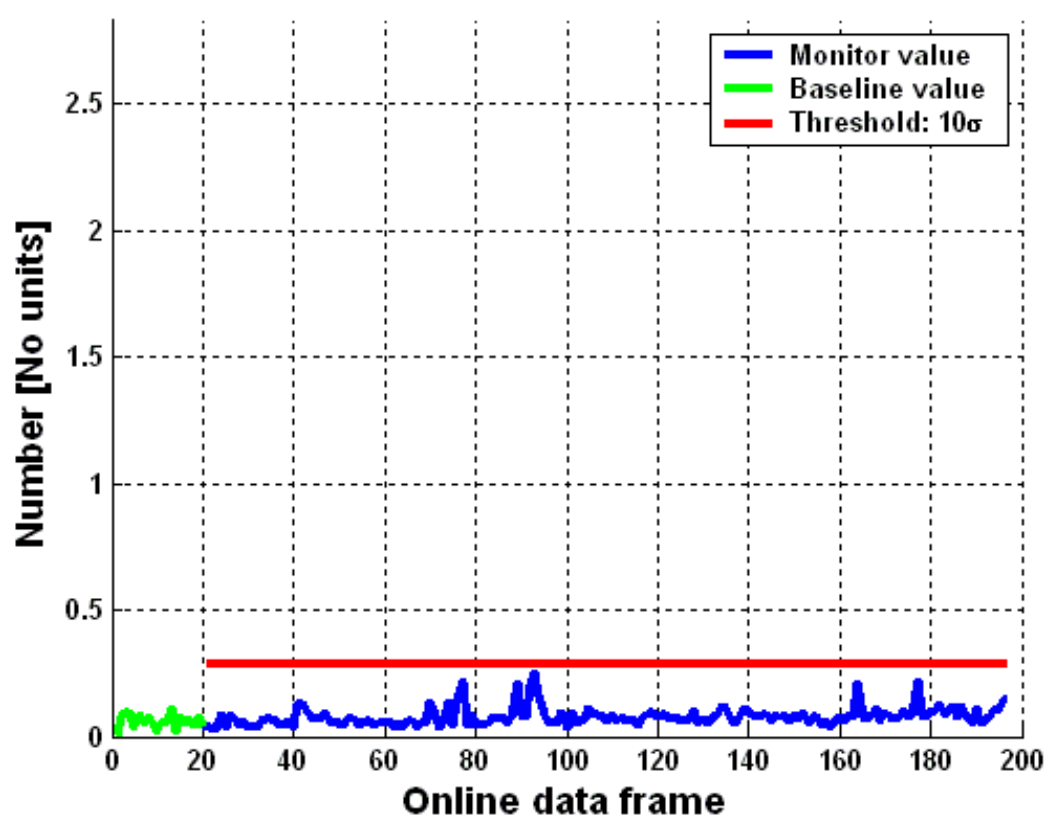

Figure 4.11 Online stability monitor 2 (Equation (4.21)) 


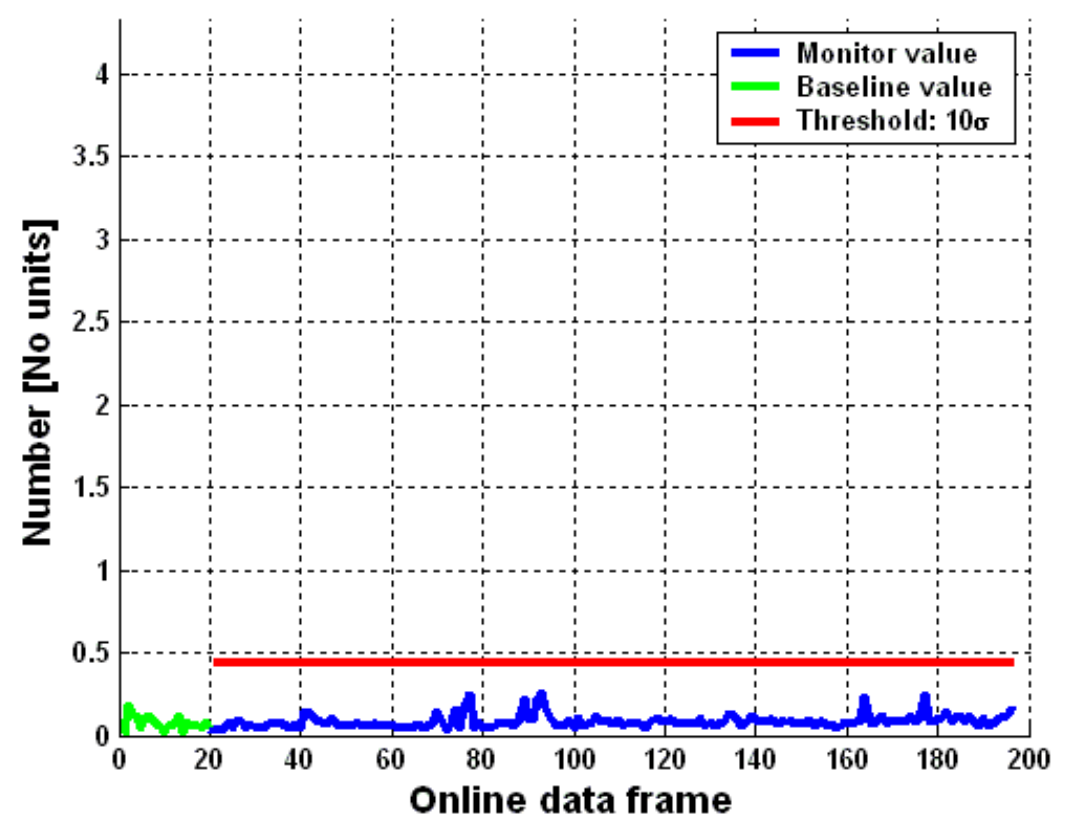

Figure 4.12 Online stability monitor 3 (Equation (4.22))

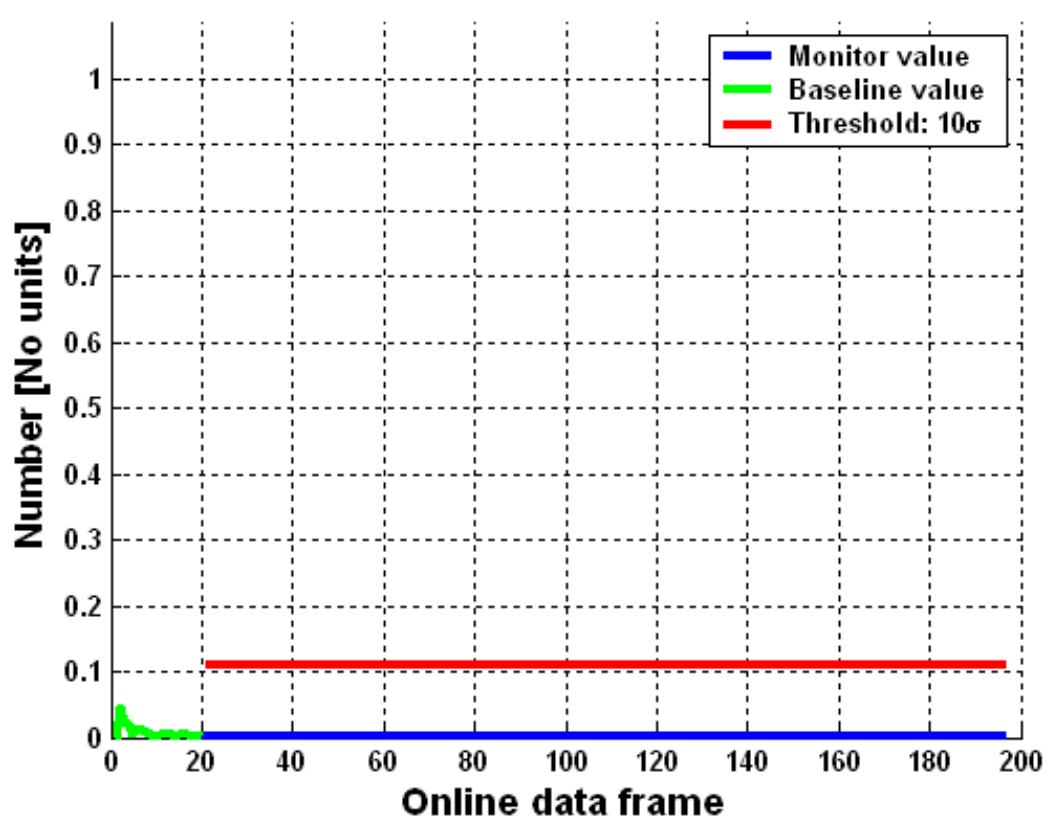

Figure 4.13 Online stability monitor 4 (Equation (4.23)) 
For this example of online learning from nominal (no-failure) flight conditions, all-4 stability monitors (stability monitors 1 (Figure 4.10), 2 (Figure 4.11), 3 (Figure 4.12), and 4 (Figure 4.13)) indicate stable online adaptation conditions. In all of the stability monitoring experiments conducted for detecting unstable online adaptation, it was observed that no single monitor was able to diagnose unstable learning behaviors for all failure scenarios. The experimental results indicate that the stability monitoring system successfully detects unstable online learning behavior in test runs using adaptive flight simulator data generated under nominal and failure flight-conditions. The results are summarized in Table 4-1, where the deviations in the monitor values after presenting the data from the cycle 100 are shown in terms of the standard deviation of the baseline monitor values, $\sigma$. Since the threshold here is set to $10 \sigma$, any deviation in the monitor value that is above $10 \sigma$ (highlighted in Table 4-1) is considered as an indication of unstable online adaptation. An indication of unstable online adaptation in any or all-4 values of the monitors indicates unstable online learning condition.

Table 4-1 Online stability monitoring results using IFCS flight data

\begin{tabular}{l|l|llll}
\hline \multicolumn{1}{c|}{$\begin{array}{c}\text { Tested } \\
\text { Flight Mode }\end{array}$} & \multicolumn{1}{|c|}{ Failed Flight Condition } & \multicolumn{4}{c}{ Deviation from Baseline [ $\sigma$ ] } \\
& & Mon 1 & Mon 2 & Mon 3 & Mon 4 \\
\hline Failure 1 & Left stabilator locked at 0 deg & 37.2 & 2.0 & 1.1 & 40.9 \\
\hline Failure 2 & Left stabilator locked at + 3 deg & 4.0 & 7.5 & 151.9 & 106.8 \\
\hline Failure 3 & Left stabilator locked at - 3 deg & 1.6 & 55.7 & 41.1 & 2.6 \\
\hline Failure 4 & Right aileron locked at + 3 deg & 1.2 & 2.1 & 2.22 & 26.5 \\
\hline Failure 5 & Right aileron locked at - 3 deg & 1.5 & 27.0 & 29.8 & 22.5 \\
\hline Failure 6 & Left stabilator missing by 50\% & 124.8 & 101.1 & 3.5 & 6.9 \\
\hline Failure 7 & Right aileron missing by 50\% & 0.6 & 1.1 & 21.6 & 16.4 \\
\hline No-failure 1 & -- & 3.3 & 3.2 & 2.4 & 0.09 \\
\hline No-failure 2 & -- & 3.26 & 3.58 & 2.65 & 0.09 \\
\hline
\end{tabular}


The three experimental results that were discussed in detail previously are based online adaptation from data that is generated under a failed flight control condition (mode 2 in Table 4-1), a failed flight control surface condition (mode 6 in Table 4-1), and no-failure flight condition (nominal mode 1 in Table 4-1) respectively. The results presented in Table 4-1 are based on several test runs of stability monitoring system using simulated flight data. The experimental study demonstrates that different monitors detect unstable online learning behavior for different failure conditions of the adaptive flight control system. This justifies our original premise of developing a multiple-monitoring based stability analysis approach.

Receiver operating characteristic (ROC) curves (otherwise known as 'relative operating characteristic' curves) were developed in the 1950's as a by-product of research into making sense of radio signals contaminated by noise $[108,109]$. In the case of the neural network stability monitoring and analysis, the use of ROC curves is to show the performance as a trade off between selectivity and sensitivity of the failure detection (stability monitoring) mechanism. As the first step, the four monitors described earlier are fused using Murphy's rule of data fusion [110]. If the value of the fused monitors is above the threshold value $(10 \sigma)$, then the neural network response is considered atypical. From Table 4-1, it can be said that in general the adaptation behavior of an embedded neural network is atypical when learning from failed flight conditions.

For each of the failed flight conditions, the failure occurs after 100 cycles of online data. In other words, the first 100 cycles of data represent data from no-failure flight conditions, and the later 100 cycles of data (cycle \# 101-200) represent data from failed-flight conditions. Since the neural network initially takes some time to settle to a stable state (observed to be about 20 learning cycles), the standard deviation of the 20 cycles of data (cycle \# 21 to cycle \# 40) is used as $\sigma$ for setting the threshold of detection. The first 20 cycles of data are considered as the monitor baseline. The 60 cycles of data (cycle \# 41 to cycle \# 100) are considered as the data generated under no-failure flight conditions, and is used to determine the false positive rate (FPR), or the probability of false alarm (PFA). The 60 
cycles of data (cycle \# 101 to cycle \# 160) are considered as the data generated under failed flight conditions, and is used to determine the true positive rate (TPR), or the probability of detection (PD).

Figure 4.14 shows the ROC curve of the fused monitors for the control failure condition: aircraft's left stabilator becomes locked at $0^{\circ}$ (failure mode 1 in Table 4-1). The area under the ROC curve in Figure 4.14 is 0.90 (a value close to 1), an indication of good performance by the monitors in detecting unstable online adaptation from the failed flight condition. Figure 4.15 shows the ROC curve of the fused monitors for the control failure condition: aircraft's left stabilator becomes locked at $+3^{\circ}$ (failure mode 2 in Table 4-1). The area under the ROC curve in Figure 4.15 is 0.91 (a value close to 1), an indication of good performance by the monitors in detecting unstable online adaptation from the failed flight condition. Figure 4.16 shows the ROC curve of the fused monitors for the control failure condition: aircraft's left stabilator becomes locked at $-3^{\circ}$ (failure mode 3 in Table 4-1). The area under the ROC curve in Figure 4.16 is 0.93 (a value close to 1), an indication of good performance by the monitors in detecting unstable online adaptation from the failed flight condition. Figure 4.17 shows the ROC curve of the fused monitors for the control failure condition: aircraft's right aileron becomes locked at $+3^{\circ}$ (failure mode 4 in Table 4-1). The area under the ROC curve is 0.98 (a value close to 1 ), an indication of good performance by the monitors in detecting unstable online adaptation from the failed flight condition. Figure 4.18 shows the ROC curve of the fused monitors for the control failure condition: aircraft's right aileron becomes locked at $-3^{\circ}$ (failure mode 5 in Table 4-1). The area under the ROC curve in Figure 4.18 is 0.98 (a value close to 1 ), an indication of good performance by the monitors in detecting unstable online adaptation from the failed flight condition. 


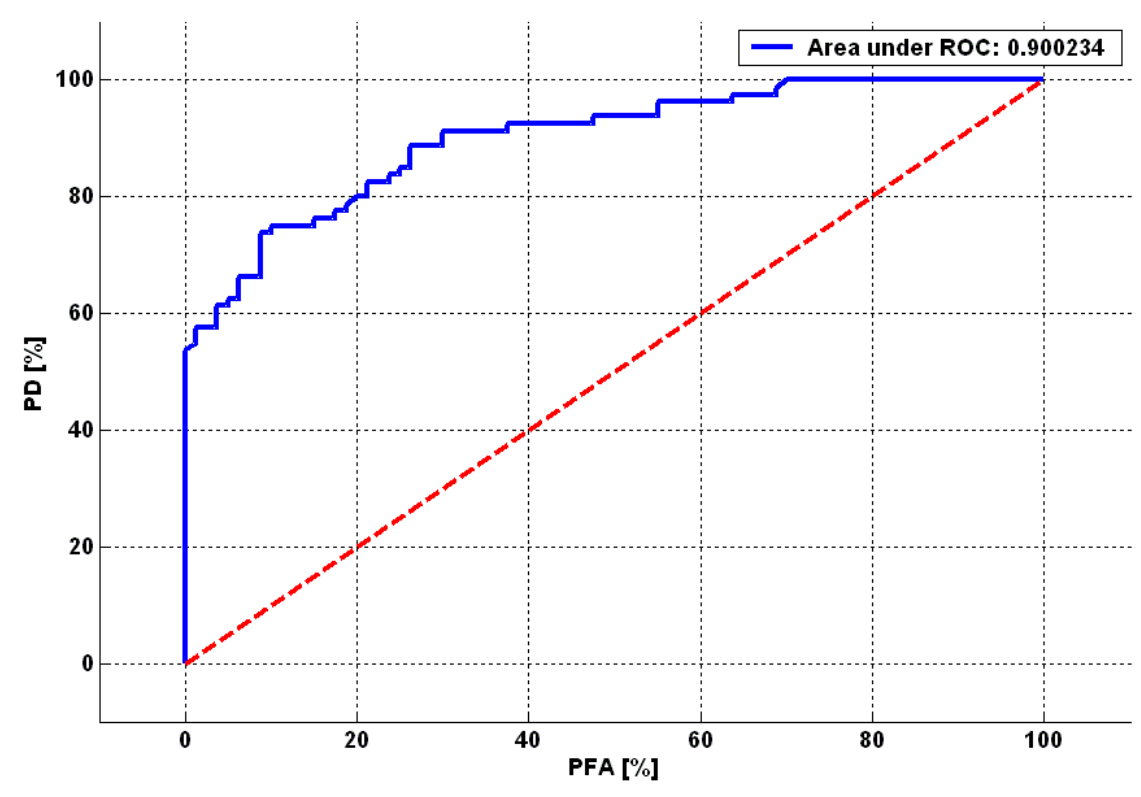

Figure 4.14 ROC curve of fused stability monitors for failure mode 1 (Table 4-1)

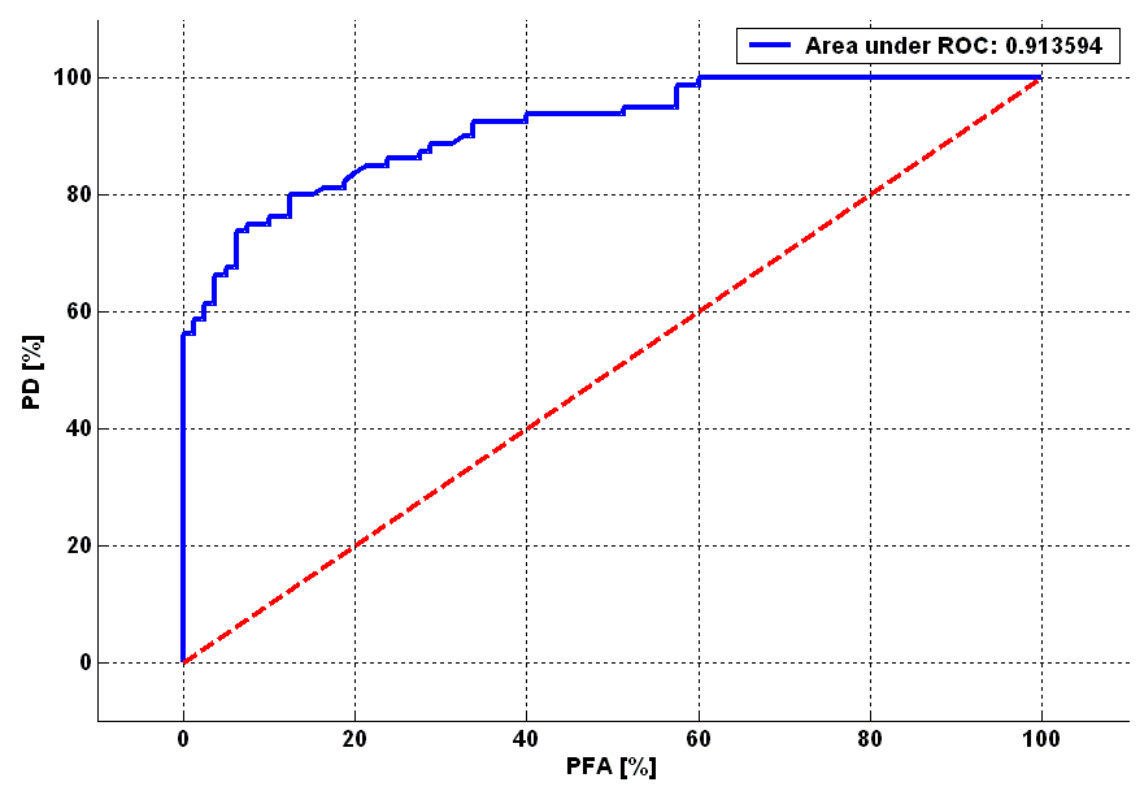

Figure 4.15 ROC curve of fused stability monitors for failure mode 2 (Table 4-1) 


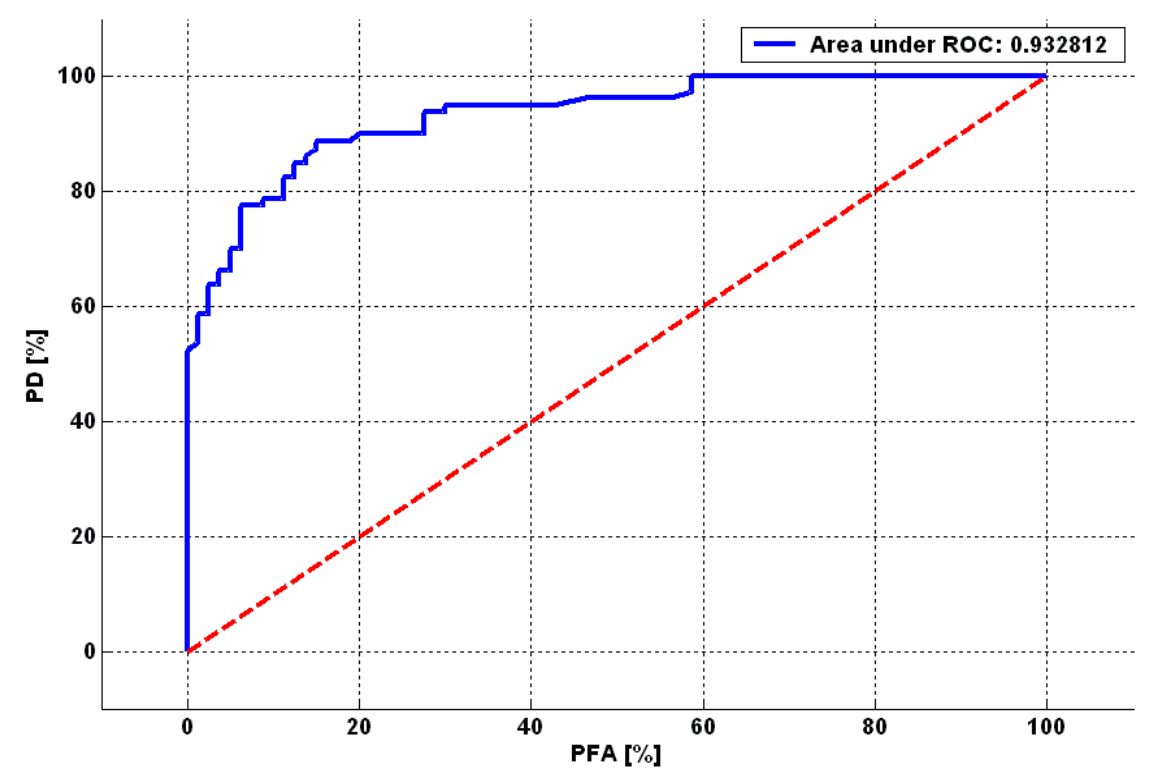

Figure 4.16 ROC curve of fused stability monitors for failure mode 3 (Table 4-1)

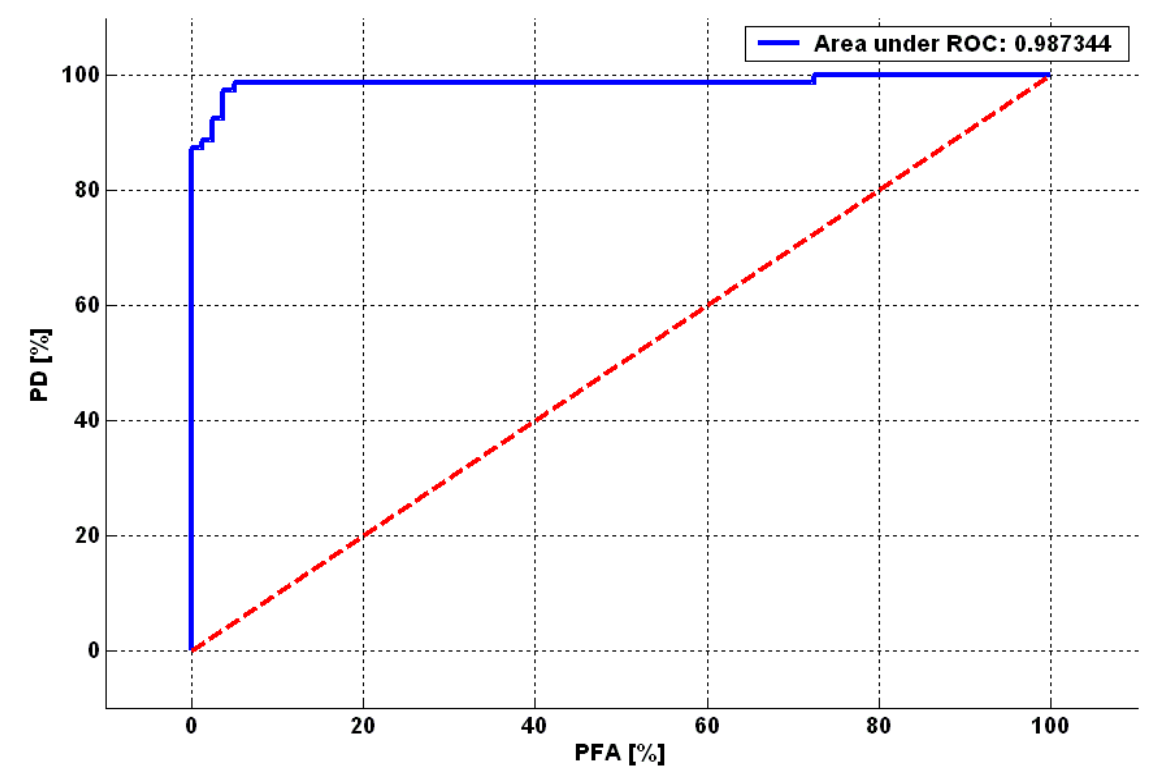

Figure 4.17 ROC curve of fused stability monitors for failure mode 4 (Table 4-1) 


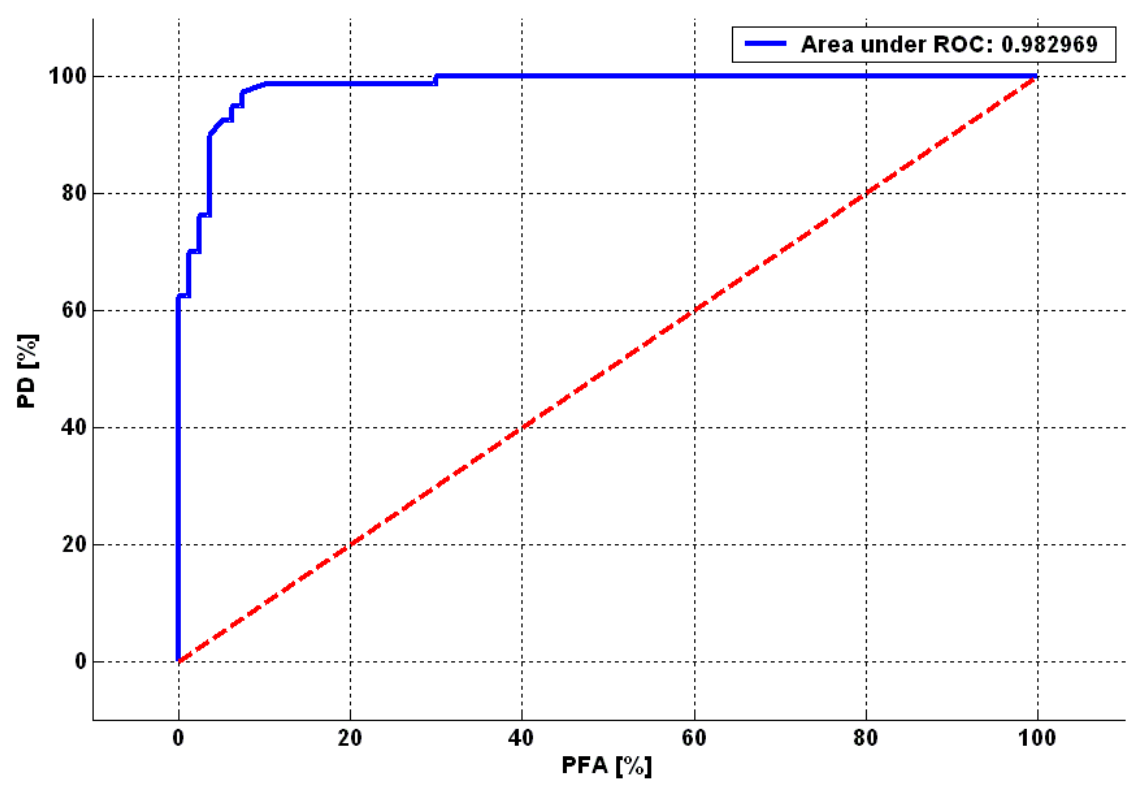

Figure 4.18 ROC curve of fused stability monitors for failure mode 5 (Table 4-1)

Figure 4.19 shows the ROC curve of the fused monitors for the control surface failure condition: aircraft's left stabilator becomes missing by 50\% (failure mode 6 in Table 4-1). The area under the ROC curve is 0.84 (a value close to 1 ), an indication of good performance by the monitors in detecting unstable online adaptation from the failed flight condition. Figure 4.20 shows the ROC curve of the fused monitors for the control surface failure condition: aircraft's right aileron becomes missing by $50 \%$ (failure mode 7 in Table 4-1). The area under the ROC curve is 0.84 (a value close to 1), an indication of good performance by the monitors in detecting unstable online adaptation from the failed flight condition.

From the ROC curves, it can be observed that the developed stability monitoring system has the capability to detect unstable online learning behavior under changing flight conditions. By making a stability monitoring system available for online use in adaptive systems, one can significantly enhance the ability to analyze and understand the behavior of the embedded adaptive software component. It is important to realize that online stability monitoring is a technique that complements theoretical stability analysis (Chapter 3 ). 


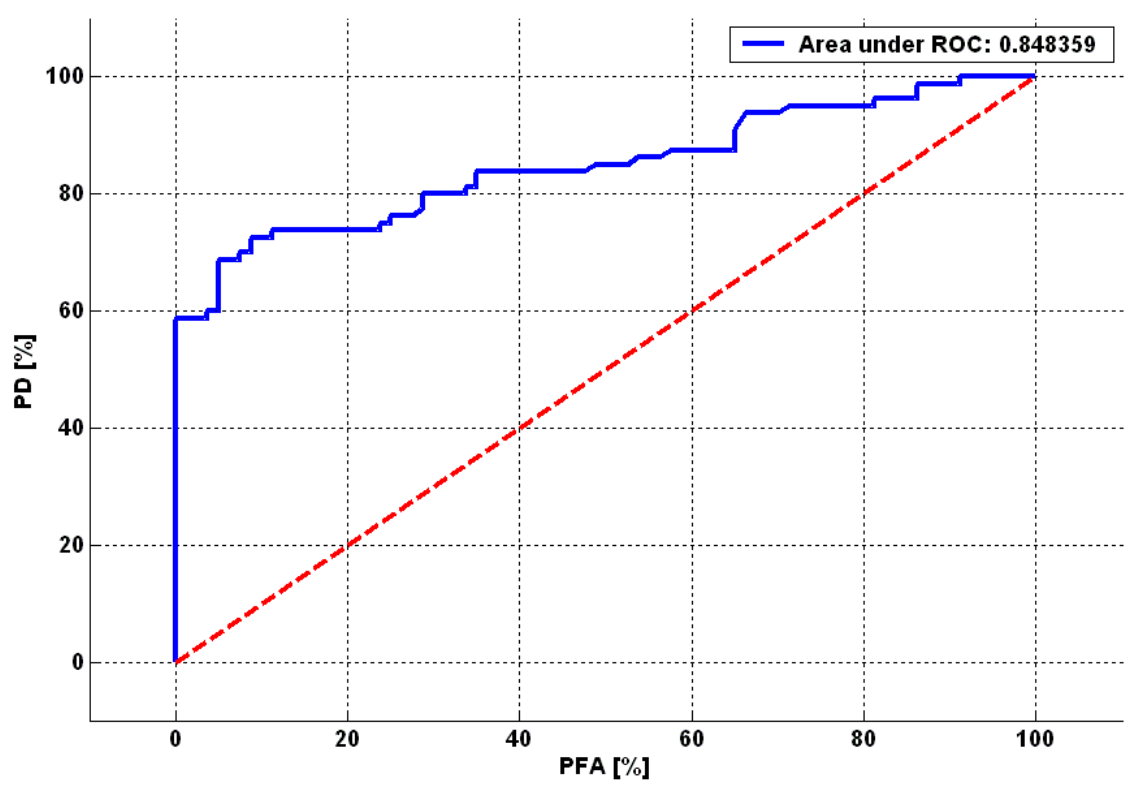

Figure 4.19 ROC curve of fused stability monitors for failure mode 6 (Table 4-1)

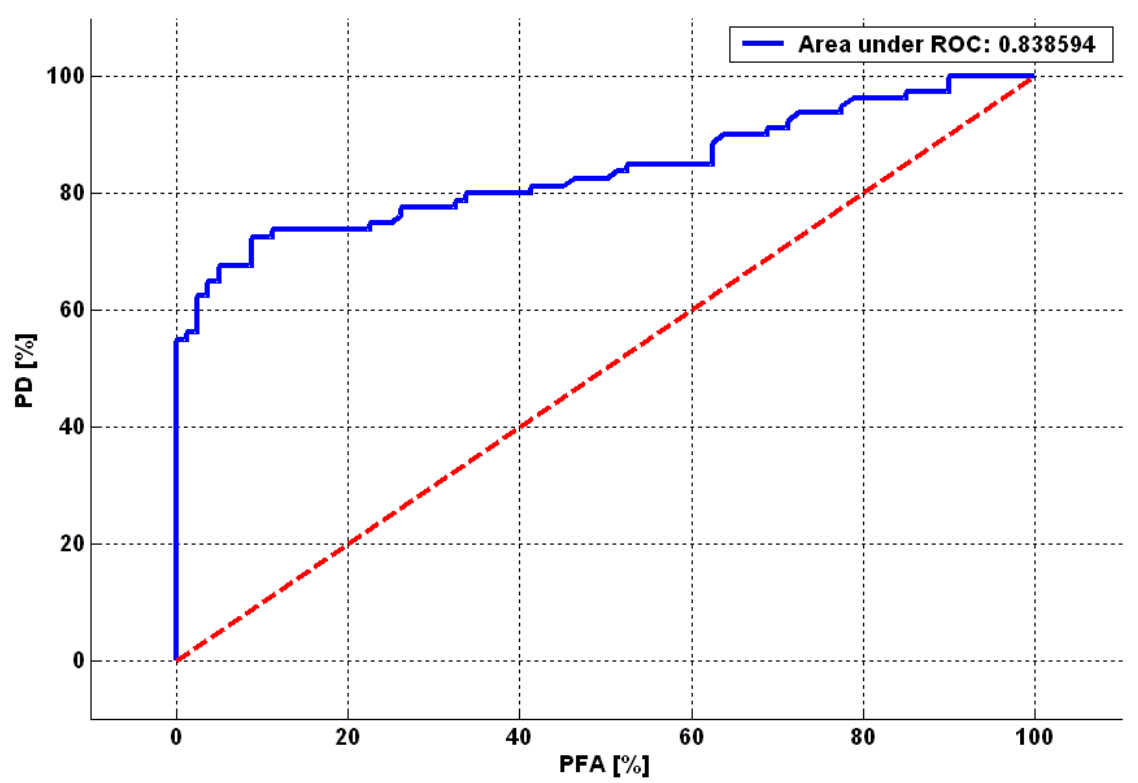

Figure 4.20 ROC curve of fused stability monitors for failure mode 7 (Table 4-1) 


\subsection{Neural Network Convergence Prediction}

In the previous section, a method for detecting the adaptation behavior of the DCS neural network to changes in the input data is discussed. The method is based on using a set of (four in total) Lyapunov inspired stability monitors that detect abnormalities in the neural network response to varying input data. The formulation of the non-quantized version of the monitors is provided in the previous Section (Equations (4.20) through (4.23)). The four monitors presented as a part of the detection mechanism are capable of detecting atypical neural network behavior due to best matching unit $(b m u)$, second best matching (sbu), neighborhood (nbr), and non-neighborhood (non-nbr) adaptation. For the sake of convenience, the quantized (or averaged) monitors are referred as follows. Qmonitor 1 represents the quantized bmu error, Qmonitor 2 represents the quantized sbu error, Qmonitor 3 represents the quantized nbr error, and Qmonitor 4 represents the quantized non-nbr error.

$$
\begin{aligned}
& \text { Qmonitor } 1=\frac{1}{N} \sum_{\mathbf{m} \in \mathbf{M}}\left\|\mathbf{m}-\mathbf{w}_{b m u(\mathbf{m})}\right\| \\
& \text { Qmonitor } 2=\frac{1}{N} \sum_{\mathbf{m} \in \mathbf{M}}\left\|\mathbf{m}-\mathbf{w}_{s b u(\mathbf{m})) \|}\right\| \\
& \text { Qmonitor } 3=\frac{1}{N} \sum_{\mathbf{m} \in \mathbf{M}} \underset{j \in\{n b r(b m u)\}}{\operatorname{avg}\left\{\left\|\mathbf{m}-\mathbf{w}_{j}\right\|\right\}} \\
& \text { Qmonitor } 4=\frac{1}{N} \sum_{\mathbf{m} \in \mathbf{M}} \underset{j \notin\{n b r(b m u)\}}{\operatorname{avg}\left\{\left\|\mathbf{m}-\mathbf{w}_{j}\right\|\right\}}
\end{aligned}
$$

The goal of convergence prediction is to be able to predict the number of cycles required by the neural network learning to return the error values (that are provided by the monitors) back to a stable state. The prediction analysis is based on the presumption that the monitors are capable of detecting real-time convergence of the neural network for changing input data streams. In order to establish a methodology of convergence prediction, first the data is artificially disturbed by the addition of data outliers. If a single point is added into the neural network data stream, then the analysis is referred as a single data-point disturbance analysis. On the other hand, the analysis due to introduction of multiple data points into the 
neural network input data stream is referred as a multiple data-point disturbance analysis. Disturbance here is referred as the introduction of either a single data point or multiple data points outside the convex hull of the neural network data set.

Due to the introduction of a single data-point or multiple data points into the neural network input data stream, the stability monitors generally indicate a spike in the error (monitor) values. The goal of convergence prediction is to provide an estimate of the number of cycles required by the neural network to return, back to a stable state. A stable state here implies a stable level of the monitor values that was observed prior the introduction of data disturbances. The concept of convergence in the four monitors (Equations (4.20) through (4.23)) is graphically illustrated here for a single point data disturbance using the example of DCS learning from twin-spiral. Disturbance here is referred as the introduction of a single data point outside the convex hull of the twin-spiral data set. In the current analysis, a single point data disturbance is introduced into the network data after 50 learning cycles. Figure 4.21 shows the convergence of Monitor 1 (Equation (4.20)) back to stability after subjecting the neural network to a single point data disturbance. Figure 4.21 indicates that it requires 33 (8350) neural network learning cycles for Monitor 1 to recover to a stable level after the introduction of a single point data disturbance. Figure 4.22 shows the convergence of Monitor 2 (Equation (4.21)) back to stability after subjecting the neural network to a single point data disturbance. Figure 4.22 indicates that it requires 34 (84-50) neural network learning cycles for Monitor 2 to recover to a stable level after the introduction of a single point data disturbance. 


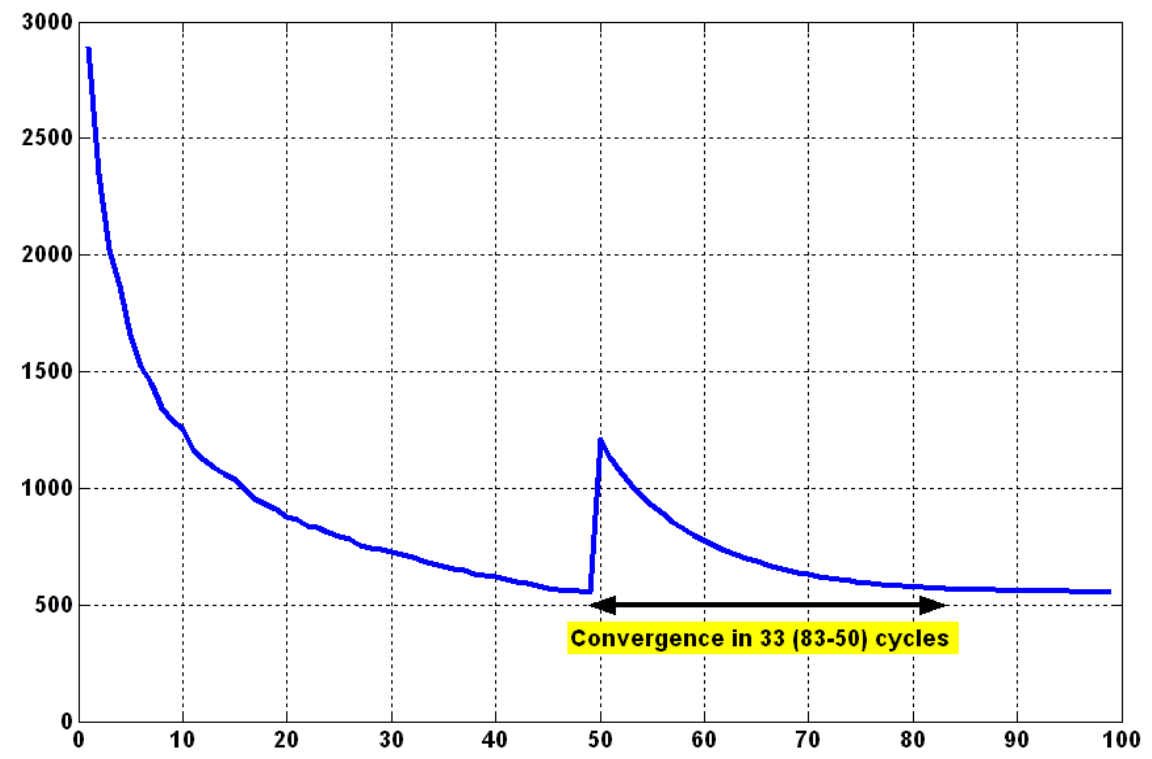

Figure 4.21 Convergence of Monitor 1 back to stability in 33 learning cycles

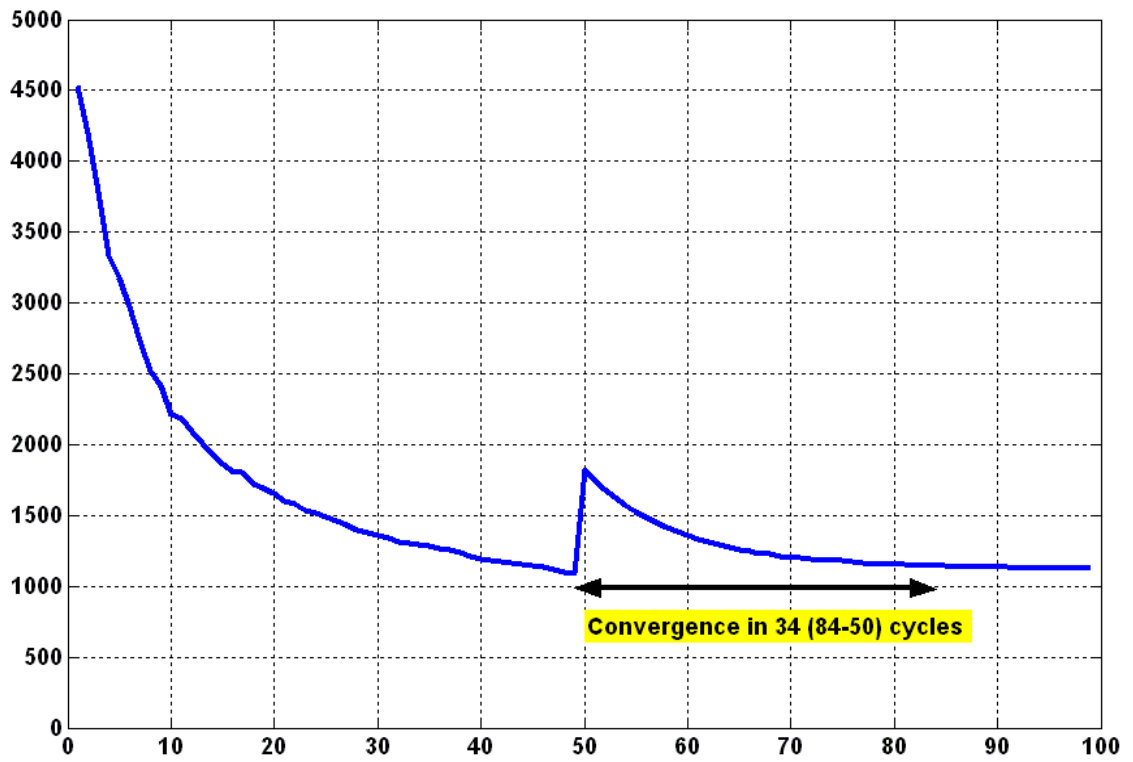

Figure 4.22 Convergence of Monitor 2 back to stability in 34 learning cycles 


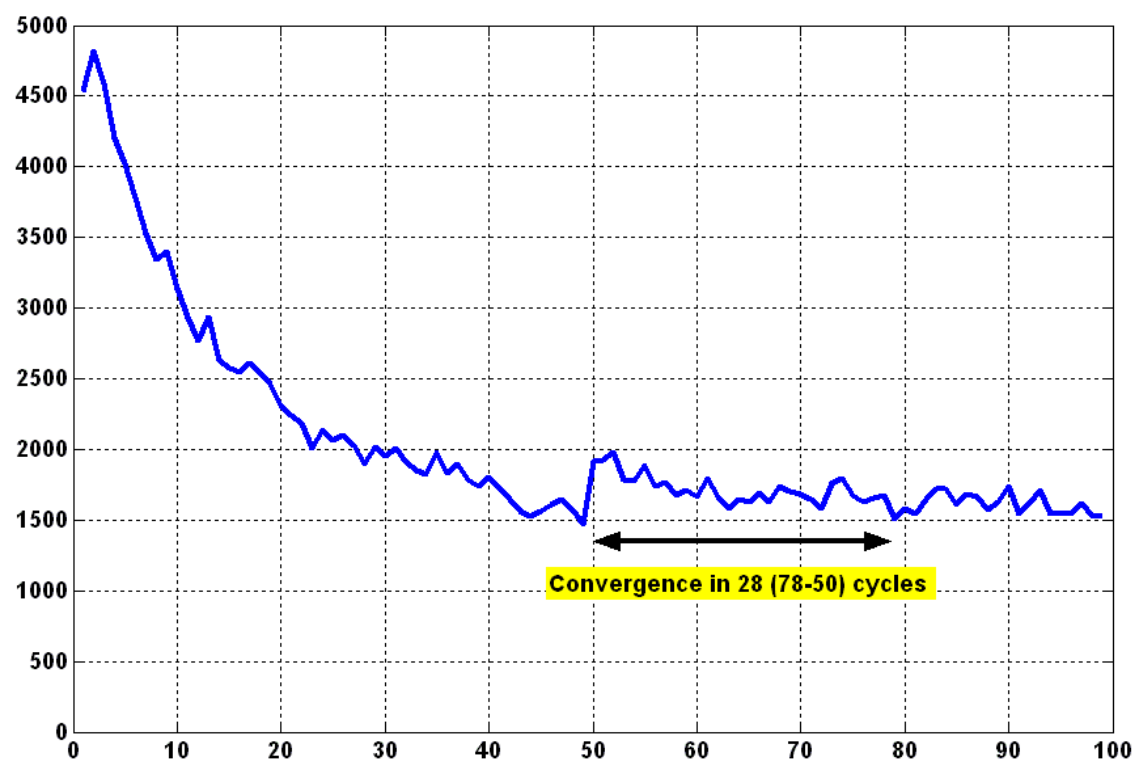

Figure 4.23 Convergence of Monitor 3 back to stability in 28 learning cycles

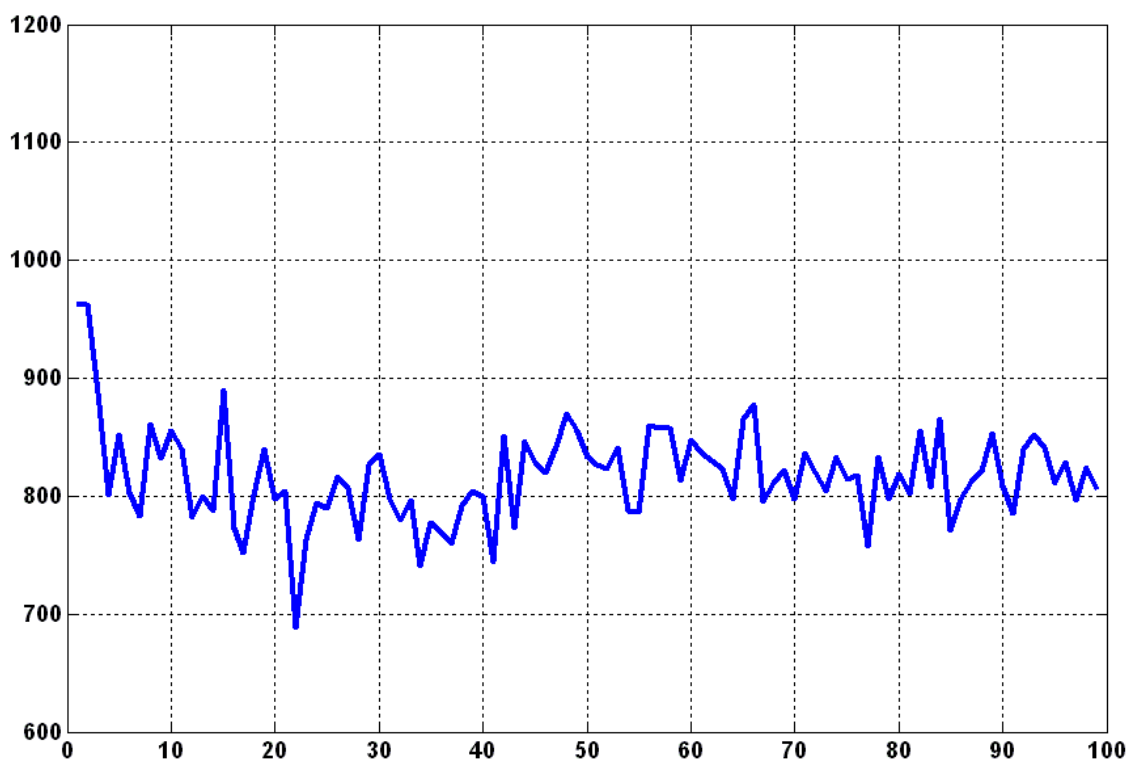

Figure 4.24 Monitor 4 shows no response to the single point data disturbance 
Figure 4.23 shows the convergence of Monitor 3 (Equation (4.22)) back to stability after subjecting the neural network to a single point data disturbance. Figure 4.23 indicates that it requires 34 (84-50) neural network learning cycles for Monitor 3 to recover to a stable level after the introduction of a single point data disturbance. Figure 4.24 shows the response of Monitor 4 (Equation (4.22)) after subjecting the neural network to a single point data disturbance. Figure 4.24 indicates that the Monitor 4 did not respond to a single point data disturbance. Therefore, for this learning example, no convergence prediction can be obtained for Monitor 4. The example also reinforces the discussion in the previous section on the need for a multiple monitoring based neural network analysis.

The analytical convergence analysis provided in the section is simplified by considering only the effects of convergence due to single point data disturbance. The analysis is further simplified by ignoring network architectures other than the Kohonen update of the best matching units and second best units. As a result, convergence predictions for only the bmu errors (Monitor 1: (4.20) and Qmonitor 1: (4.24)), and sbu errors (Monitor 2: (4.21) and Qmonitor 2: (4.25)) for single point data disturbances are considered in the analysis. This is because the nbr errors (Monitor 3: (4.22) and Qmonitor 3: (4.26)), and non-nbr errors (Monitor 4: (4.23) and Qmonitor 4: (4.27)) are highly dynamic in nature as they are based solely on the network connectivity structure. It should be realized that network connectivity structure changes frequently and it is difficult to provide an accurate prediction for the convergence of the $n b r$ and non-nbr errors.

\subsubsection{Convergence Prediction of the BMU Errors}

In this Section, the effects of the single-point data disturbance due to the best matching unit adaptation, the bmu errors (Monitor 1: (4.20) and Qmonitor 1: (4.24)) are considered. In this analysis an attempt is made to anticipate the number of training cycles required by the neural network to converge the error levels of the bmu errors (Monitor 1 and Qmonitor 1) to previously observed stable error levels from single point data disturbances. 
The disturbances, in this case, occur after the corresponding bmu errors have reached stable error levels.

Let Monitor 1 (Equation (4.20)) be denoted by $E_{B M U}$ and let Qmonitor 1 (Equation (4.24)) be denoted by $Q_{B M U}$. Note that $Q_{B M U}$ represents the quantized (or average) of the bmu error, $E_{B M U}$. Let $\xi_{B M U}$ be the Kohonen constant for the bmu update. Let $m$ be an input element of the training data set, $M \subset \mathfrak{R}^{D}$ and let $W_{B M U}$ represent the weight center of the $b m u$ of the element $m$. Let $E_{B M U}$, the error due to the $b m u$ update measure as

$$
E_{B M U}=\sum_{m \in M}\left\|m-w_{B M U}\right\|
$$

Let $E_{B M U}^{o}$ represent the value of the bmu error, $E_{B M U}$ just before the addition of a single point data element, $m^{*}$ into the training data set, $M \subset \mathfrak{R}^{D}$. In other words, $E_{B M U}^{o}$ represents the bmu error of the network just before the application of the single point data disturbance.

Let $Q_{B M U}$ be the quantized (or average of the) bmu error for the DCS network measure as

$$
Q_{B M U}=\frac{1}{N} \sum_{m \in M}\left\|m-w_{B M U}\right\|
$$

In Equation (4.29), $N$ represents the number of nodes in the DCS network. Let $Q_{B M U}^{o}$ be the value of the quantized bmu error just before the addition of a single point data element, $m^{*}$ into the training data set, $M \subset \mathfrak{R}^{D}$. In other words, $Q_{B M U}^{o}$ represents the quantized bmu error just before the application of the single point data disturbance.

Let $E_{B M U}^{*}$ be the perturbation error caused by the addition of the data element, $m^{*}$ into the training data set, $M \subset \mathfrak{R}^{D}$.

$$
E_{B M U}^{*}=\left\|m^{*}-w_{B M U}\right\|
$$


The following analytical result provides a prediction estimate of the number of neural network learning cycles required in order for the bmu error, $E_{B M U}$ to converge back to a stable level.

\section{Theorem 4.1 Convergence of the BMU Error}

If a data point, $m^{*}$ is added into the data set, $M \subset \mathfrak{R}^{D}$ after $N_{0}$ learning cycles, then the DCS best matching unit error, $E_{B M U}$ will return to the previous stable error level, $E_{B M U}^{o}$ in $k$ learning cycles such that

$$
k<\frac{\log \left(\frac{E_{B M U}^{o}}{N_{o} E_{B M U}^{*}}\right)}{\log \left(1-\xi_{B M U}\right)}
$$

Proof A detailed mathematical proof is provided in the Appendix

Similar analytical result concerning the convergence prediction of the quantized $b m u$ error $Q_{B M U}$ can be provided. The quantized bmu error $Q_{B M U}$ is a commonly used stopping criterion for implementations of the DCS network. For this analysis, a comparison is performed in the way the Kohonen rule decreases error relative to the learning cycles to the quantized error. The following analytical result provides a prediction estimate of the number of neural network learning cycles required in order for the quantized BMU error, $Q_{B M U}$ to converge back to a stable level.

\section{Theorem 4.2 Convergence of the Quantized BMU Error}

If a data point, $m^{*}$ is added into the data set, $M \subset \mathfrak{R}^{D}$ after $N_{0}$ learning cycles, then the quantized best matching unit error, $Q_{B M U}$ in the DCS will return to the previous stable error level, $Q_{B M U}^{o}$ within $k$ learning cycles provided

$$
\frac{\left(1-\xi_{B M U}\right)^{k}}{k}<\frac{E_{B M U}^{o}}{N_{o} E^{*}{ }_{B M U}}
$$

Proof A detailed mathematical proof is provided in the Appendix 
For this result, note that solving for $k$ explicitly in (4.32) yields a Lambert w-function so that the value of $k$ must be determined by cycling through values until the left hand side matches the right. This complication arises due to the linear use of $k$ in the denominator of the quantized error combined with the exponential use in the Kohonen update term. This emphasizes the fact that the result for the bmu error ignores the growth of the network explicitly, and as a result should yield longer times for returning to the unperturbed total error numbers whereas the latter result directly uses both network growth and the Kohonen correction and should therefore yield smaller re-stabilization times. This behavior is what is observed experimentally.

In the 50 learning experiments that were conducted using the twin-spiral data, the DCS was subjected to single point data disturbances. In each of the 50 learning experiments, a single point data was randomly injected into the twin-spiral data in different locations after the network adapts to the twin-spiral data for 50 learning cycles. The convergence back to stability in the bmu error (Monitor 1, Equation (4.20)), and the quantized bmu error (Qmonitor 1. Equation (4.24)) errors were recorded. An algorithm is implemented to predict the convergence in the bmu error and the quantized bmu error. The implementation of the algorithm is based on the analytical results presented previously (Theorem 4.1 and Theorem 4.2). Figure 4.25 shows the results of predicting the convergence in the bmu error (Monitor 1 , Equation (4.20)) using the implemented convergence prediction algorithm. In Figure 4.25, the average and maximum of the error in predicting the convergence of the bmu error using the implemented convergence prediction algorithm is 5 and 11 learning cycles respectively.

Figure 4.26 shows the results of predicting the convergence in the quantized bmu error (Qmonitor 1, (4.24)) using the implemented convergence prediction algorithm. In Figure 4.26, the average and maximum of the error in predicting the convergence of the quantized bmu error using the implemented convergence prediction algorithm is 0.5 and 1 learning cycles respectively. 


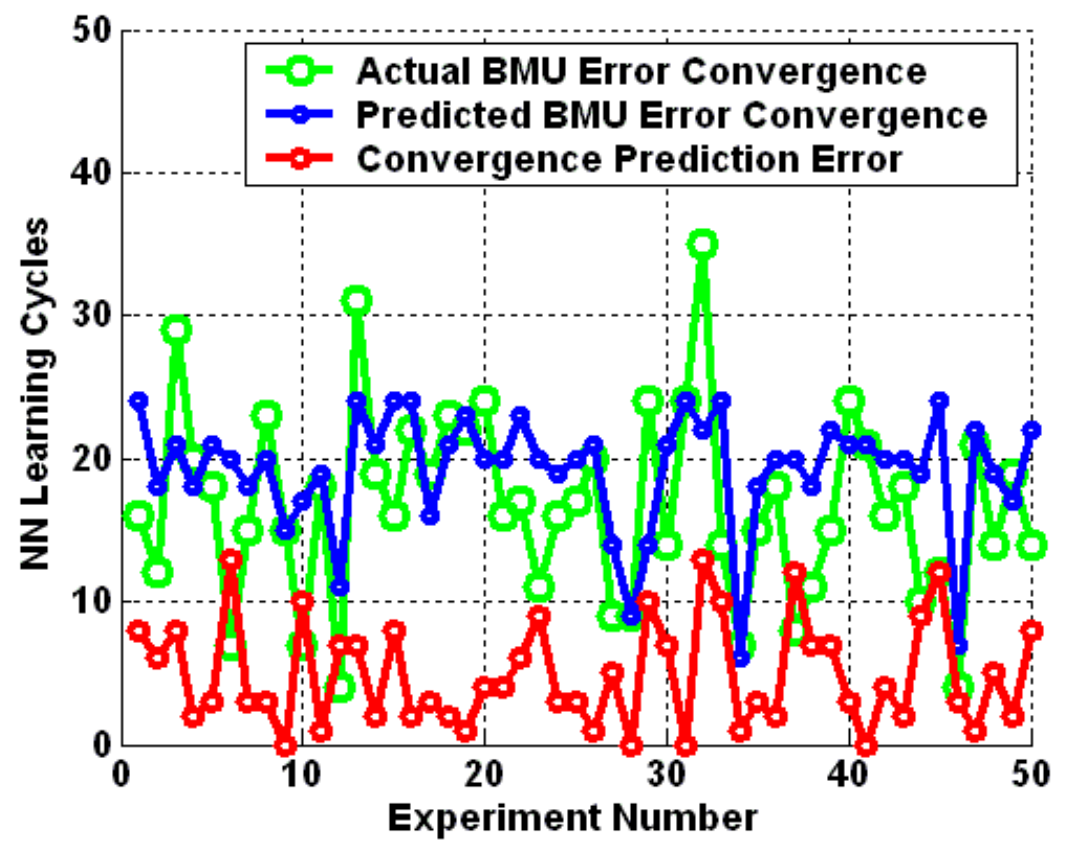

Figure 4.25 Results from predicting convergence in the BMU error

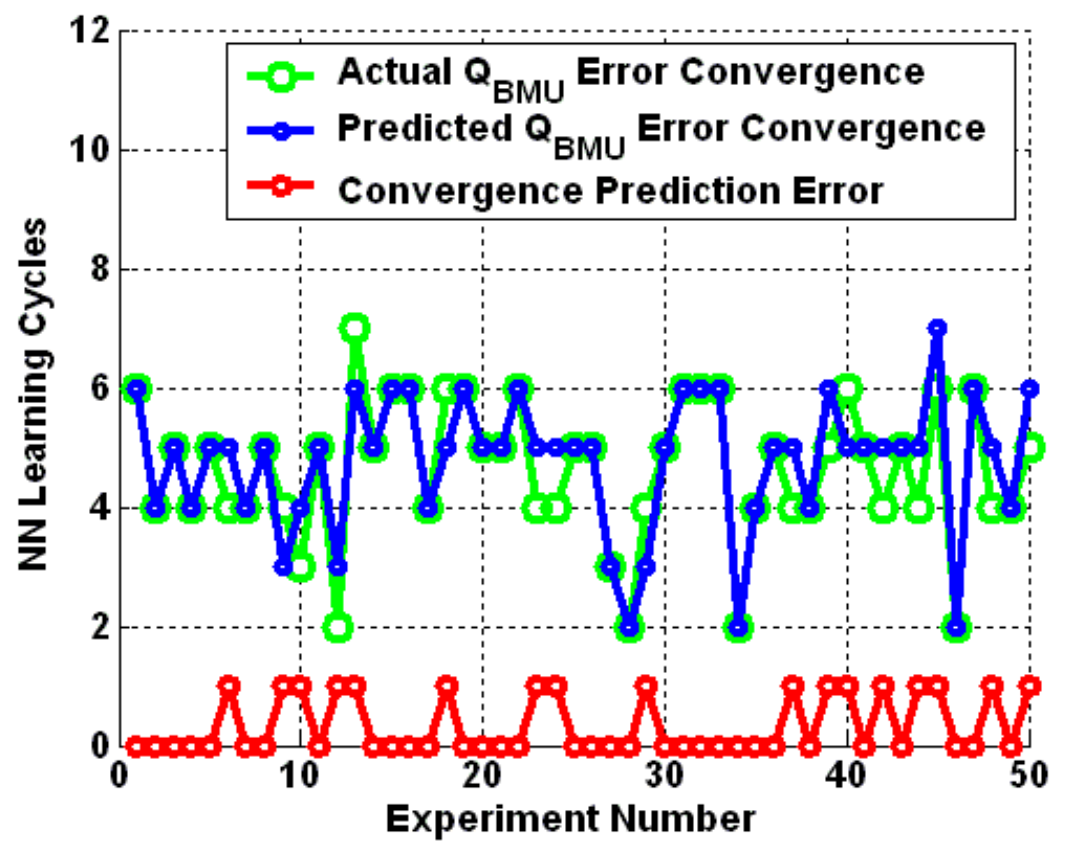

Figure 4.26 Results from predicting convergence in the quantized BMU error 
A comparison of Figure 4.25 and Figure 4.26 makes it evident that the quantized $b m u$ error (Figure 4.26) converges to a stable state in fewer learning cycles in comparison to its counterpart, the bmu error (Figure 4.25). It can also be observed from Figure 4.25 and Figure 4.26 that the quantized bmu error (Figure 4.26) is less sensitive to single point data disturbances and converges faster to a stable level than the bmu error (Figure 4.25). 


\section{Chapter}

\section{Improving Learning Efficiency in $\mathbf{N}$}

Topology preservation is an important property of information processing useful in many practical applications including exploratory data analysis, control systems, signal processing, image processing, pattern recognition, speech analysis, and computational topology. Topology preservation, in simplified terms, can be described as a projection where neighboring (or similar) input data patterns are mapped onto neighboring (or similar) nodes (to the extent that it is possible). Topology preservation ensures that connected structures remain connected and the neighborhood (or closeness) relationship is retained.

Topology preserving neural networks like the Kohonen Self-Organizing Maps (KSOMs) are characterized by the ability to extract information encoded in the input data of highdimension into features using prototypes, called weight vectors (also: reference vectors, codebook vectors) $[22,37]$. K-SOM models, in general, consist of a 2-dimensional lattice of connected nodes (also: neurons, units), where each node is associated with a weight vector. After presenting an input data pattern to all nodes, a similarity matching is performed to determine the weight vector that best matches the presented input vector. Similarity matching in $\mathrm{K}-\mathrm{SOMS}$ is the process of finding the node whose weight vector has the minimum distance from the input vector $[19,22]$. The Euclidean metric is the most commonly used distance measure for similarity matching. The node that is closest to the presented data pattern is referred as the best matching unit or the winning unit. The weight vectors of the best matching unit and its neighboring units are allowed to update. The update process is performed such that the selected weight vectors move closer to the presented input data pattern. In this manner, the network learns a representation of the input data. In K-SOMs, the nodes are allocated according to the probability distribution that is described by the input data and the topological ordering reflects the structure of the input data patterns [24]. 
A major drawback of $\mathrm{K}-\mathrm{SOMs}$ is that they require predetermination of the network structure and dimensionality. It has been realized that an optimal selection of the network dimensionality and structure can only be determined after accounting for the statistical properties of the input data [32]. As in most cases of unsupervised learning, the unavailability of this information can result in mappings from K-SOMs that represent the input space less accurately $[32,37]$. Another drawback of K-SOMs is that the network size (number of nodes) must be specified in advance (before initiating the learning). The network remains fixed throughout the learning process, and can limit the learning capacity of K-SOMs, making them unsuitable for learning from continuous or non-stationary data distributions [32]. These drawbacks have motivated the proposition and development of a number of variations of $\mathrm{K}$ SOM models that favor dynamic allocation of network size and/or shape. Growing selforganizing maps (G-SOMs) are one of the most prominent types of K-SOM variations that represent a class of neural networks with a variable network size and shape [30, 37].

The growing cell structures (GCS) neural networks proposed by Fritzke in [31, 34]. have a variable network size (number of nodes increase or decrease), and an arbitrarily chosen fixed network dimensionality. In the growing neural gas (GNG) model (also proposed by Fritzke in [30]), the network size is changed (usually increased) during the self-organization process. GNG models combine the concept of growing self-organizing networks (originally proposed by Fritzke for GCS), and the competitive Hebb learning rule that is proposed by Martinetz and Schulten [37]. The main difference in the GNG networks is the topology of the network, which is allowed to evolve during learning, and not constrained to a fixed dimensionality as in the GCS neural networks. The growing grid (GG) model is another type of incremental self-organizing neural network [35]. The network generated by the GG model is a rectangular grid of certain dimensionality. The basic concepts of growing self-organizing networks used in GCS and GNG are applied (with some modifications) in GG networks to a growing rectangular grid [35]. Bruske and Sommer have embarked on a more dynamic approach for allocating network size and shape in growing self-organizing networks and proposed the dynamic cell structures (DCS) $[17,18]$. The DCS networks combine the concept 
of growing self-organization (originally proposed for GCS), and the competitive Hebb learning rule (with some modifications form the originally proposed GNG networks). Additional details regarding the implementation and the topology preserving nature of the DCS networks will be discussed in Section 5.1.

Most of the G-SOM models described in the literature, including the neural networks described above, are based on dynamic allocation of network size and shape. In essence, these dynamic allocation algorithms allow the network size and shape to evolve during the generation of a feature map. The evolution of the feature map is based on successive addition (and sometimes, occasional deletion) of network nodes [17, 18, 30, 35]. Some form of local neural network error information associated with each node, and is accumulated at the node during the adaptation process. After a certain number of adaptation steps (usually a fixed number in the case of off-line learning), the dynamic allocation algorithms add either a single node or an entire layer of nodes into the network. This process is repeated until the network model satisfies the stopping criterion. A stopping criterion can be for example, an upper limit to the network size, or a predetermined total neural network approximation error [17, 18, 30, 35]. In this manner, dynamic allocation algorithms overcome the problem of determining the optimal network size and structure before initiating learning. The feature of dynamic allocation of network size and shape allow G-SOMs to extract information encoded in the input data more accurately, and more commonly with a fewer number of nodes than traditional K-SOMs with a predefined network size and shape [17, 18, 30, 35].

The selection of an appropriate neural network growing strategy for dynamic allocation of network size and shape is crucial for achieving topology preservation in G-SOMs $[32,37]$. It has been observed that current neural network growing strategies suffer from two fundamental flaws in their implementation. Most neural network growing strategies for GSOMs that are currently available in the literature are based on addition of nodes into the network between the node with the highest accumulated local error and its connected node (neighbor) $[17,18,30,35]$. While this process of dynamic allocation of network size and shape is, in general, shown to generate effective topology preserving mappings from a variety of 
input data distributions, it is shown here and in [36] that certain conditions of the data and/or the network can prompt growing strategies to add nodes into regions that represent no data. It is important to realize that introduction of nodes into regions representing no data can not only disrupt the topology preservation properties of the neural network, but also increase the computational effort on the neural network training algorithm to recover back to a topology preserving mapping. This is because many neural network growing strategies fail to account for the distribution of the input data during the addition of nodes. Further details regarding the conditions of the data and/or the network that lead to a disruption in topology preserving properties of the neural network, the types of disruptions, and the neural network recovery back to topology preservation will be discussed in Section 5.1. Secondly, some existing neural network growing strategies for dynamic allocation of network size and shape are observed to suffer from the following flaw in the implementation of the algorithm. Under certain conditions of the neural network, the neuron with highest accumulated error is observed as being disconnected from the network. In other words, a neighbor for the node with the highest accumulated error may not always exist. Such a condition can result in an implementation error for neural network growing strategies that are based on addition of nodes in between the node with the highest accumulated error and its connected neighbor. Additional details regarding the nature of the implementation error, and the reason for its occurrence will be discussed in the Section 5.1. Since it is not possible to discuss the details of the conditions leading to these two flaws for all types of G-SOMs, the analysis here is limited in discussion to the DCS neural networks $[17,18]$. The concepts and issues presented here are kept generic and apply to other G-SOMs.

It may be of less priority to investigate the topology preserving properties of selforganizing maps that are used in standard neural network applications. However, for safety critical applications of neural networks such as path navigation in unmanned aerial vehicles (UAVs), advanced flight control systems, and intrusion detection in computing security, it is necessary to ensure that the SOM is capable of generating a topology preserving mapping throughout the neural network's learning process. For example, in the NASA's intelligent flight 
control system (IFCS), the DCS neural networks are used in Phase I of the project to approximate the aircraft system dynamics for improving the aircraft's performance and control $[40,42,49]$

Based on the observed problems with current dynamic allocation algorithms, a modified neural network growing algorithm for dynamic allocation of network size and shape for G-SOMs is presented in this section. The proposed modifications allow the presented dynamic allocation algorithm to add nodes strictly into regions of the network containing data, and more commonly, where the necessity for additional neurons is most required. The modified neural network growing algorithm first selects the appropriate region of the network where the necessity for additional neurons is most required. The selection of the appropriate region is based on the accumulated local error, but other selection criteria can be used. The modified dynamic allocation algorithm then adds nodes into the selected regions by overlaying them on top of data patterns that are located further away from the centers of the masked Voronoi regions. Finally, the modified neural network growing algorithm updates the lateral connections between the nodes by applying the competitive Hebb rule using the data patterns that belong to the selected masked Voronoi regions. Some major modifications in the implementation of the modified neural network growing process are listed as follows.

1. As opposed to adding nodes into regions of the network between the nodes with highaccumulated local error, the modified neural network growing process adds nodes into network regions surrounding the nodes with high-accumulated local error by overlaying them over certain data patterns that are not properly represented by the neural network.

2. Before entering into another cycle of neural network adaptation, the lateral connections between the newly introduced node and the nodes in the vicinity are updated using the competitive Hebb rule.

The first modification allows the dynamic allocation algorithm to introduce nodes strictly into regions that represent data. The second modification ensures that the newly introduced neuron forms a part of the existing neural network map. These modifications allow 
the presented neural network growing algorithm to overcome the two problems of dynamic allocation of network size and shape that were discussed earlier. While the motive behind the development of the modified dynamic allocation algorithm was to overcome the problems existing with current neural network growing strategies, it was observed here that the modified algorithm not only overcomes these problems, but also able to generate more efficient topology preserving mappings throughout the neural network learning process. Detailed mathematical deirvations are presented in the section to validate the claim.

Section 5.1 provides a series of formal mathematical derivations to describe the conditions of the neural network and the input data that lead to a disruption in the topology preserving properties of the DCS self-organizing map. In Section 5.2, a cyclework for efficient dynamic allocation of network size and shape is described followed by the presentation of the modified neural network growing strategy. Section 5.2 also provides a series of formal mathematical derivations indicating that the modified neural network growing strategy has a potential for efficient dynamic allocation of network size and shape in growing self-organizing maps. An example of the DCS neural network learning using the original and modified neural network growing algorithms is discussed in Section 5.2 to compare their topology preserving properties.

\section{1. $\quad$ Conditions Leading to Loss of Topology in SOMs}

Most of the G-SOM models including, the GCS, GG, GNG, and DCS are based on dynamic allocation of network size and shape. In essence, the dynamic allocation processes in these networks allow the neural network size and shape to evolve during the generation of a feature map. The evolution of the feature map is based on successive addition (and sometimes, occasional deletion) of network nodes [17, 18, 30, 35]. A form of local neural network approximation error is associated with each node, and is accumulated at the node during the adaptation process. In the case of the DCS, the distance between the input data pattern and the corresponding best matching unit, also called as the resource value, is used for measuring the local error. After a certain number of adaptation steps (usually a fixed 
number in the case of off-line learning), the dynamic allocation algorithms add either a single node or an entire layer of nodes into the network [17, 18]. This process is repeated until the network model satisfies a predetermined stopping criterion. The average of the local error, commonly known as the quantization error is used in the DCS as the neural network approximation error.

$$
Q E=\frac{1}{N} \sum_{m \in M}\left\|m-W_{B M U(m)}\right\|
$$

If the Quantization error (5.1) is above a predetermined threshold, meaning that there is a need for an additional neuron in the network, then a new node is added into the selforganizing map [17, 18, 30, 35]. In the DCS, and in other G-SOMS such as the GCS, GG, and GNG, the location of the additional node is between the node with the highest resource value and its connected node (neighbor) with the highest resource value. These nodes are called as the highest resource neuron (hrn) and the second highest neuron (shn) respectively. The objective of introducing an additional node in a region of the network between nodes with higher resource values is to lower the resource value, and hence reduce the overall neural network approximation error (average of the resource values). In this manner, the dynamic allocation algorithm in the DCS overcomes the problem of determining the optimal network size and structure before initiating learning [17, 18, 30,35]. While the details of the neural network growing algorithms for other G-SOMS (GCS, GG, GNG) are omitted for brevity, the process of dynamic allocation network size and shape in other G-SOMS is very similar to that in the DCS [17, 18, 30,35]. In other words, though the analytical results presented here are based on the learning dynamics of the DCS, in general the concepts that are discussed here apply to other G-SOMs as well. Following are the details of the steps followed in the neural network growing process of the DCS. Recall that the details of the DCS neural network training algorithm are discussed in Section 2.2, in Chapter 2.

Step 1 Locate the node of the network that has the highest resource value, $r(h r n)=\max \{r(i)\}, \forall i \in\{1,2, \mathrm{~K}, N\}$, where $r(i)$ represents the resource value of the node $i$ in 
the DCS SOM of network size, $N$. Let $W($ hrn $) \in W$ be the weight center of the highest resource value node.

Step 2 Locate the neighbor (connected node) of the highest resource value node with the highest resource value, also referred as the second highest resource value node, $r(s h n)=\max \{r(j)\}, \forall j \neq h r n \in\{1,2, \mathrm{~K}, N\}, C(h r n, j)>0$, where $r(j)$ represents the resource value of the node $j$ in a SOM network of size $N$. Let $W(\operatorname{shn}) \in W$ be the weight center of the second highest resource value node.

Step 3 Allocate a weight center to the new node, $W($ new $) \in W$ between the weight centers of the highest and the second highest resource value nodes, $W(\mathrm{hrn})$ and $W(\mathrm{shn})$ respectively

Note that $W(h r n)$ and $W(s h n)$ are obtained from Step 1 and Step 2 respectively. The exact location of the weight center of the new node is based on the resource values of the highest and the second highest resource value nodes, $r(h r n)$ and $r(s h n)$ respectively.

$$
\begin{gathered}
R R(h r n)=\frac{r(h r n)}{r(h r n)+r(s h n)} \\
R R(s h n)=\frac{r(s h n)}{r(h r n)+r(s h n)} \\
W(\text { new })=W(h r n) R R(h r n)+W(s h n) R R(s h n)
\end{gathered}
$$

where $R R(h r n)$ and $R R(s h n)$ represent the resource ratios of the highest and the second highest resource value nodes respectively. From (5.2) and (5.3) it is evident that the following holds.

$$
R R(\operatorname{shn})+R R(h r n)=1
$$

Step 4 Assign the newly introduced node a resource value. The resource values of the highest and the second highest resource value nodes are redistributed among the highest and second highest resource value nodes, and the newly added node. 


$$
\begin{aligned}
& \Delta r(h r n)=\frac{1}{2} R R(h r n) r(h r n) \\
& \Delta r(s h n)=\frac{1}{2} R R(s h n) r(s h n) \\
& r(n e w)=\Delta r(h r n)+\Delta r(s h n) \\
& r(h r n)=r(h r n)-\Delta r(h r n) \\
& r(s h n)=r(s h n)-\Delta r(s h n)
\end{aligned}
$$

According to Step 2 and Step 3 of the neural network growing process, a new node is added into the self-organizing map in a region between the highest resource value node and its (connected) neighbor with the highest resource value. It is shown here that the node with the highest resource value can be disconnected from every other node of the network. In other words, the following mathematical derivation shows that there may not be any neighbors to the highest resource value node.

\section{Theorem 5.1 Disconnected Highest Resource Node}

There is a positive probability that the node with the highest resource value is disconnected from every other node of the neural network

Proof A detailed mathematical proof is provided in the Appendix section.

The significance of the above analytical result (Theorem 5.1) is that it indicates that the process of dynamic allocation of network size and shape followed in the DCS networks suffer from a fundamental flaw in their implementation. The specific conditions that lead to this implementation error in the process of dynamic allocation of network size and shape are limited in this analysis to the DCS self-organizing neural networks for brevity. However, it must be emphasized that similar conditions can lead to implementation errors in the dynamic allocation process of network size and shape for other G-SOMs such as the GCS, GG, and GNG. This is because the current processes of dynamic allocation of network size and shape in G-SOMS is based on the addition of nodes between the nodes with the highest resource value (accumulated local error) and their (connected) neighbors.

In the DCS (and in most other G-SOMs such as the GCS, GG, and GNG), new nodes are introduced into the network in regions between the node with the highest resource value 
and its (connected) neighbor with the highest resource value, also called as the second highest resource value neuron. The motive behind this process of dynamic allocation of network size and shape is the following. Resource values represent local error measures of the nodes in a network and the addition of nodes in regions closer to the nodes with a high resource value is expected to lower the average resource value of the network, the quantization error in (5.1). A major problem underlying this process of dynamic allocation of network size and shape in SOMs is that the resource value of the network is assumed to represent the distribution of the input data. However, it is shown in the following mathematical derivation that dynamic allocation processes that add nodes into regions using only the resource value information and not take into consideration the distribution of the input data can result in addition of nodes into network regions that represent no data. More importantly, it will be shown here that a processes of dynamic allocation of network size and shape that add nodes into a network region that represents no data can lead to loss in the topology preserving properties of the self-organizing map. Before proceeding any further, let us formalize the description of a topology preserving mapping. The definition of a topology preserving mapping that is provided here is based on the work of Matrinetz and Schulten in [37].

A topology preserving feature map is determined by a mapping, $\Phi$ from the input manifold, $M \subset \mathfrak{R}^{D}$ onto the nodes (vertices of the SOM), $i \in\{1,2, \mathrm{~K}, N\}$ of $G$, the selforganizing map. The mapping $M \rightarrow G$ is determined by neural weight centers $W(i) \in W, i \in\{1,2, \mathrm{~K}, N\}$ of the vertices of the SOM. An input data pattern, $m \in M$ is mapped to a node, $i=b m u, i \in\{1,2, \mathrm{~K}, N\}$, the weight center $W(b m u) \in W$ of which is closest to $m \in M$. In other words, a data pattern $m$ of the input manifold $M$ is mapped to the node $i$ of the SOM, $G$ whose Voronoi polyhedron $V(b m u(m))$ encloses $m$, where $b m u(m)$ represents the best matching unit of the input data pattern $m \in M$. The mapping 
$\Phi$ is completely determined by the weight centers, $W(i) \in W, i \in\{1,2, \mathrm{~K}, N\}$ and it can be written that

$$
\Phi_{W}: M \rightarrow G, m \in M \rightarrow b m u(m) \in G
$$

The mapping $\Phi_{W}$ is neighborhood preserving if input data patterns that are close in $M$ are mapped to nodes that are close in the SOM, $G$. This implies that the weight centers that are neighbors (or close) in the SOM, $G$ are assigned to nodes that are adjacent in $M$. The inverse mapping $\Phi_{W}^{-1}$ from the SOM, $G$ to $M$ is given as

$$
\Phi_{W}^{-1}: G \rightarrow M, i \in G \rightarrow W(i) \in M
$$

The mapping $\Phi_{W}^{-1}$ is neighborhood preserving if weight centers of adjacent nodes are neighboring in the manifold $M$. The self-organizing map, $G$ with vertices, $i \in\{1,2, \mathrm{~K}, N\}$ is said to have formed a topology preserving mapping, if the mapping $\Phi_{W}$ from $M$ to $G$, and the inverse mapping $\Phi_{W}^{-1}$ from $G$ to $M$ are neighborhood preserving [37]. This implies that the connection structure of the SOM, $G$ is comparable to the connection structure, $M$ and vice versa to determine whether a given map is topology preserving. It is important to realize from this definition the criticality of the lateral connection structure for the generation of a topology preserving mapping. We shall now proceed to the analytical result. The result presented here shows that a process of dynamic allocation of network size and shape that is based on addition of nodes into regions between higher resource value neurons, and do not take into consideration the effect of the distribution of the input data, is an inefficient way to generate a topology preserving mapping.

\section{Theorem 5.2 Loss of Topology Preserving Properties}

The process of dynamic allocation of network size and shape in SOMs that is based completely on resource values (or other local error measures) for addition of nodes into the network (usually between higher resource value neurons) does not always generate a topology preserving mapping.

Proof A detailed mathematical proof is provided in the Appendix section. 
It is important to realize that introduction of nodes into regions representing no data can not only disrupt the topology preserving properties of the SOM, but it can also increase the computational effort on the neural network training algorithm to recover back to a topology preserving mapping. The influence of dynamic allocation of network size and shape on the computational effort on the neural network training will be discussed using examples in following section, Section 5.2. The discussion also indicates that dynamic allocation of network size and shape should not be completely based on the resource values (or any other local error) as the error measure does not always encapsulate the actual topology of the network with respect to the presented data. To overcome this problem, a modified neural network growing process is presented in the following section, which adds nodes into the network by taking into account the distribution of the input data along with the network's resource values.

\subsection{An Improvised Neural Network Growing Process}

Based on the observed problems with existing dynamic allocation algorithms (also discussed in the previous section), a modified neural network growing algorithm for dynamic allocation of network size and shape for G-SOMs is presented in this section. Modifications are proposed to enable the neural network growing algorithm to overcome the two problems that were discussed in the previous section. More importantly, the proposed modifications allow the dynamic allocation process to add nodes strictly into regions of the network containing data, and more commonly, where the necessity for additional neurons is most required. The steps followed in the modified neural network growing algorithm are as follows.

Step* 1 Locate the node of the network that has the highest resource value, $r(h r n)=\max \{r(i)\}, \forall i \in\{1,2, \mathrm{~K}, N\}$, where $r(i)$ represents the resource value of the node $i$ in the DCS SOM of network size, $N$. Let $W($ hrn $) \in W$ be the weight center of the highest resource value node.

Step $^{*} 2$ Identify the set of input data patterns that have the node with the highest resource value, $i=h r n \in\{1,2, \mathrm{~K}, N\}$ as their best matching unit, referred as the membership set, $\{m(h r n)\} \in M$. 
From the membership set, $\{m(h r n)\}$, locate the data pattern that is far most from the weight center of the highest resource value node, $m^{*} \in\{m(h r n)\}$. Set the weight center of the new node as the location of the data pattern, $W($ new $)=m^{*}$.

Step* 3 Update the lateral connections between the nodes using the competitive Hebb rule. However, instead of updating the connections for all input data patterns, use only the input data patterns that have the highest resource value neuron as their best matching, the membership set of highest resource value node, $\{m(h r n)\}$.

Step* 4 Locate all nodes that are connected to the newly added node, $i \in\{$ nbr $($ new $)\} \in\{1,2, \mathrm{~K}, N\}, \forall C(i$, new $)>0$. Compute the Data Resource Ratios, $D R R(i)$ and the resource adjustments, $\Delta r(i)$ for each element of the non-empty set, $\{$ nbr $($ new $)\}$ as shown below. Assign a resource value to the newly introduced node.

Let $n$ be the total number of input data elements in the membership set, $\{m(n b r)\} \in M$, obtained from Step* 2. Let $n(i)$ be the number of input data patterns that have the node, $i \in\{n b r(n e w)\}$, the (connected) neighbor(s) of the highest resource value node as their best matching units. For each $i \in\{n b r(n e w)\}$,

$$
\begin{aligned}
& D R R(i)=\frac{n(i)}{N} \\
& \Delta r(i)=\frac{r(i) D R R(i)}{\sum i} \\
& r(n e w)=\sum \Delta r(i) \\
& r(i)=r(i)-\Delta r(i)
\end{aligned}
$$

where $\sum i$ is the number of elements in the set $i \in\{n b r($ new $)\}$, the total number of neighbors of the newly added node. $r(i)$ represents the resource value of node $i$ that is an element of the set $\{n b r(n e w)\}$. 
The first step of the modified neural network growing algorithm (Step* 1 ) is similar to the first step of the original growing algorithm (Step 1). In the second step of the original neural network growing algorithm (Step 2), the neighbor of the highest resource value node with the highest resource value (referred as the second highest resource value node) is located. In the previous section (in Theorem 5.1), it was shown that the second step of the original neural network growing process (Step 2) is vulnerable to implementation errors. This is because the highest resource value node can sometimes become disconnected from the network (Theorem 5.1). It is also discussed in the previous section (in Theorem 5.2) that the highest resource node can sometimes be connected to nodes that are non-adjacent in the input manifold $M$. In such a case, the addition of a node into the network regions between the highest and second highest resource value nodes is shown to result in addition of nodes into regions representing no data (Theorem 5.2). In other words, it leads to the generation of a neural network map, $G$, that is not topology preserving with respect to the input manifold $M$.

To overcome the problems encountered in neural network growing processes, in the second step of the modified neural network growing process (Step* 2$)$, the set of input data patterns that have the node with the highest resource value as their best matching unit are located. The set of data patterns is collectively referred to as the membership set of the highest resource value node. From the membership set of the highest resource value node, the data pattern that is located further away from the masked Voronoi center (the weight center of the highest resource value node) is located. A node is added into the SOM by overlaying it over this data pattern. Note that the second step of the modified neural network growing process $\left(\right.$ Step $\left.^{*} 2\right)$ relies on the membership set (data distribution) of the highest resource value node rather than relying solely on the network topology (connectivity structure). This modification avoids the previously observed implementation errors, as it is able to add nodes into appropriate regions of the network even when the highest resource value neuron is disconnected from the network. It will be shown, shortly, that due to addition of nodes on top of data patterns in this manner, the network error in the modified neural network growing 
process will always be lower than the network error in the original neural network growing process.

In the third step of the modified neural network growing process (Step* 3 ), the lateral connections between nodes are updated using competitive Hebb rule. However, instead of updating the connections for all input data patterns (as done in the inner loop of the neural network learning algorithm), the update is performed using only the data set that has the highest resource value node as the best matching unit (the membership set obtained from Step* 2). This modification ensures that the newly introduced node is connected to at least one other node of the network, and more frequently in a topology preserving manner.

Finally, the newly added node is allocated with a resource value in a manner that is similar to the implementation of the original neural network growing process (Step* 4). A notable difference in the implementation of the original neural network growing process is that the newly added node is restricted in connection to two nodes, the highest and second highest resource value nodes. However, in the modified neural network growing algorithm, the newly added node becomes connected to at least one, but there is no restriction to the maximum number of nodes that can be connected to the newly added node. The number of nodes connected to the newly added node is flexible and is driven by the input data distribution and the resource values of nodes. This is the difference between the final step of the modified neural network growing algorithm (Step* 4$)$, and the final step of the original neural network growing algorithm (Step 4).

The major differences between the modified and the original process of dynamic allocation of network size and shape are summarized as follows.

- Nodes are added into the neural network by overlaying them over certain data patterns that are not properly represented by the neural network, and not between two nodes as in the original growing strategies. 
- Before entering into another cycle of neural network adaptation, the lateral connections between nodes are updated by applying competitive Heb rule in a modified manner as described above.

The first modification allows the dynamic allocation algorithm to introduce nodes into network regions that represent data. The second modification ensures that the newly introduced node forms a part of the existing neural network map. These modifications allow the presented neural network growing algorithm to overcome the two problems of dynamic allocation of network size and shape that were discussed earlier. The original motive behind the development of the modified dynamic allocation algorithm was to overcome the problems existing with current neural network growing strategies. However, as shown in the following mathematical derivation, the presented algorithm not only overcomes these problems, but is also observed to generate more efficient topology preserving mappings throughout the neural network learning process.

\section{Theorem 5.3 Efficient Topology Preservation}

A neural network growing strategy that is based on addition of nodes into network regions between higher resource value nodes results in a neural network error that is higher than that caused as a result of using a neural network growing strategy that is based on addition of nodes into network regions that are overlaid over input data patterns.

Proof A detailed mathematical proof is provided in the Appendix section.

The mathematical derivation shows that using the modified neural network growing algorithm results in a network error that is lower than the network error due to a dynamic allocation process that is based on adding nodes into network regions between nodes such as the original neural network growing algorithm. This is because the modified growing strategy introduces additional nodes into the SOM by overlaying them over input data patterns and applies competitive Hebb rule to ensure that the newly introduced neuron becomes a part of the topology (connectivity structure) of the existing network. On the other hand, the original neural network growing strategy, makes an assumption that the existing SOM is neighborhood preserving and, hence, the introduction of a node into a region between the higher resource 
value neurons leads to a topology preserving mapping. However, the mathematical derivation given in Theorem 5.2 shows that this need not always be the case. Thereby, the triangulation formed by the original neural network growing algorithm need not always reduce the cumulative error of the masked Voronoi region, and can sometimes disrupt the topology preserving properties of the SOM. In a general sense, the mathematical derivation applies for any process of dynamic allocation of network size and shape that is based on a similar concept of adding nodes into the network by overlaying then over data patterns.

It is important to realize that introduction of nodes into regions representing no data can not only disrupt the topology preservation properties of the SOM, but can also increase the computational effort on the neural network training algorithm to recover back to a topology preserving mapping. In order to demonstrate the effectiveness of the modified neural network growing process in terms of generating accurate topology preserving neural net mappings throughout the leaning process, consider the following example of the DCS neural net selforganization from a twin-spiral data manifold using the original and the modified neural network growing process. The reason for choosing the twin-spiral data as the neural net input data for the comparison analysis is that the twin-spiral function represents a classical dataclustering problem [18]. The twin-spiral data shown in Figure 5.1 consists of 962, twodimensional data patterns and serves as the DCS neural net input data.

The mappings generated by the DCS neural net using the original and modified neural network growing algorithms shall be compared to each other. For this comparison study, the original neural network growing strategy followed in the DCS (Step 1, Step 2, Step 3, and Step 4) serves as the original neural network growing process, which is compared to the modified neural network growing process described previously in the section (Step* 1 , Step* 2 , Step* 3 , and Step* 4). The twin-spiral, neural net input data shown in Figure 5.1 is collected from the Carnegie Mellon University's artificial neural network benchmark data repository $[66,67]$.

In the comparison analysis, the topology of the mappings generated by the original and the modified neural network growing algorithms are compared to the topology of the input data (twin-spiral function). In order to provide a fair comparison between the two dynamic 
allocation algorithms, the random probabilities that select the input data patterns from the input manifold for SOM adaptation are kept the same. Note that since we are dealing with the DCS, a growing self-organizing map (G-SOM), an adaptation cycle (or an epoch) represents the SOM adaptation to all input data patterns, i.e. a complete sweep of the training data set (962 samples of the twin-spiral data) followed by an introduction of a new node into the network.

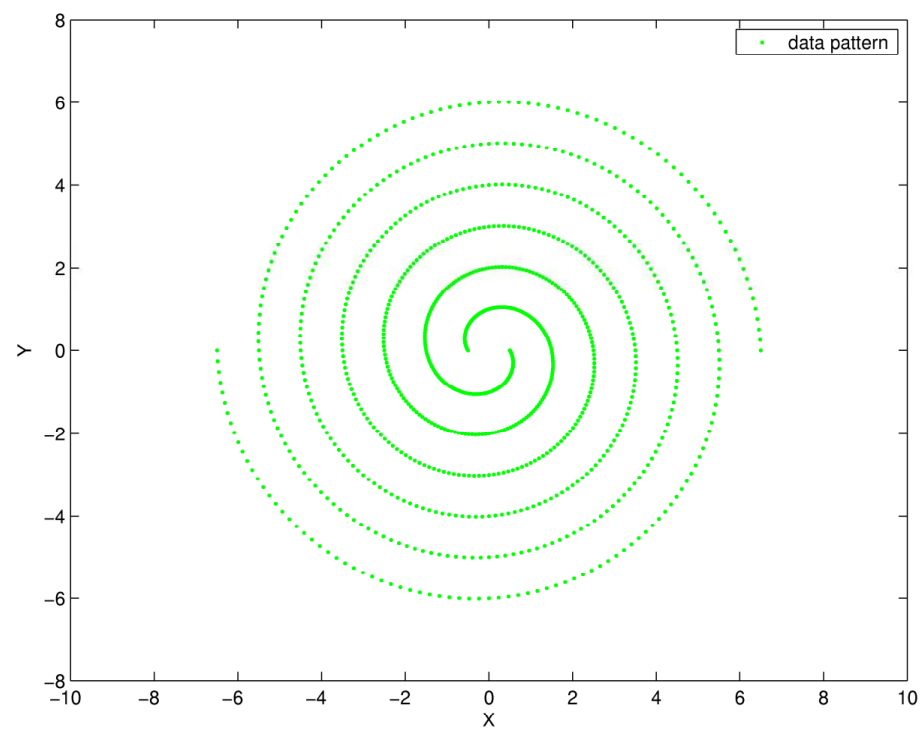

Figure 5.1 Twin-Spiral Neural Network Input Data

Figure 5.2 and Figure 5.3 show the snapshots of the DCS SOM representations of the twin-spiral data using the original and the modified neural network growing algorithms respectively after the network size reaches to 114 nodes (after 114 cycles of adaptation). In Figure 5.2 and Figure 5.3, the weight centers of the nodes are indicated using darker colored dots and the twin-spiral data patterns are shown in the background using lighter colored dots. Note that the snapshots of the SOM shown in Figure 5.2 and Figure 5.3 are at relatively early stages of the neural network adaptation (114 cycles) with respect to the size of the presented twin-spiral data (962 data patterns). 


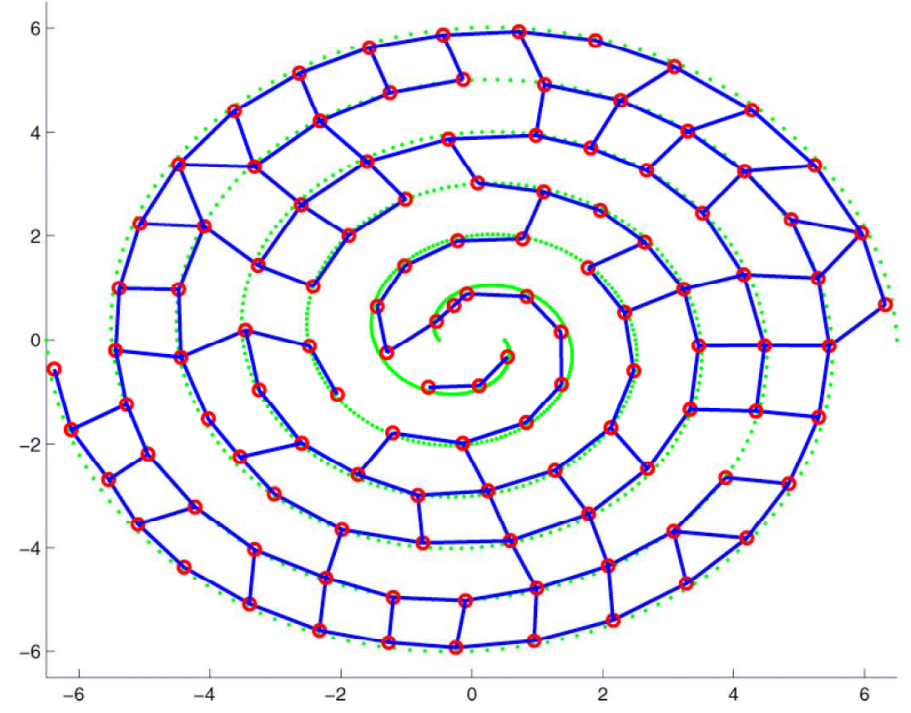

Figure 5.2 DCS using the original NN growing algorithm after 114 cycles

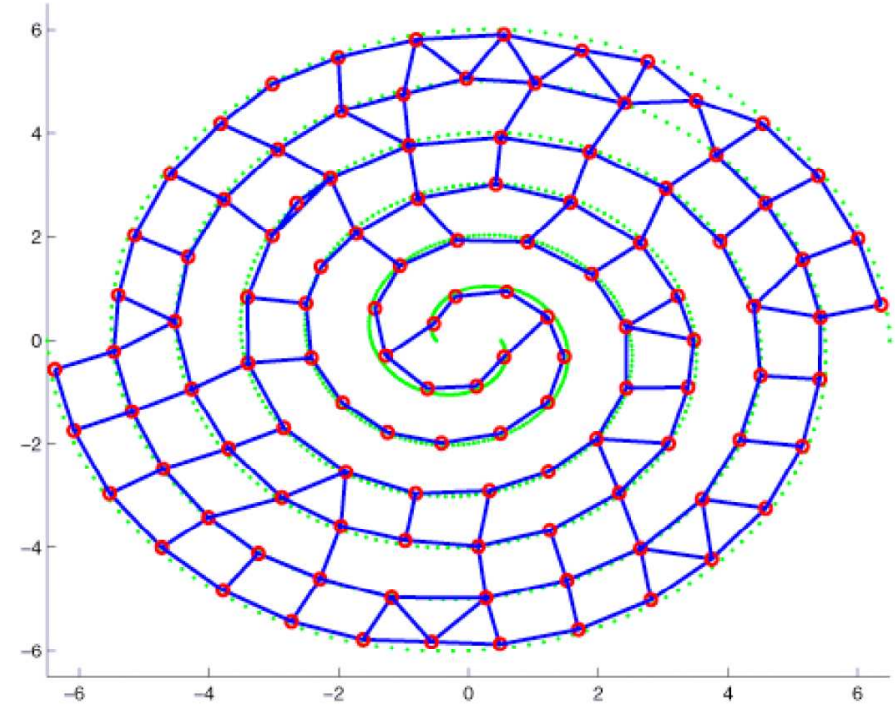

Figure 5.3 DCS using the modified NN growing algorithm after 114 cycles

Figure 5.4 and Figure 5.5 show the snapshots of the DCS SOM immediately following the addition of a new node, node \#115 into the network using the original and the modified neural network growing algorithms respectively. As indicated by the circle ' $A$ ' in Figure 5.4, the original growing algorithm introduces the new node (node \#115) into a region within the 
network between the two spirals that does not represent any data. This is because the neural network growing strategy in the original growing algorithm is based on addition of nodes into regions of the network between the highest and second highest resource value nodes. In this case, the highest and second highest resource value nodes happen to be non-adjacent nodes, representing two different spiral functions. The map generated in Figure 5.4 can, therefore, be considered as a non-topology preserving mapping as the neighborhood relationship is not maintained. As shown in Figure 5.5, the modified neural network growing algorithm introduces the new node (node \#115) into a network region that represents data in a topology preserving manner. This is because the neural network growing strategy followed in the modified growing algorithm overlays the new node over the far most data pattern that has the highest resource value node as its best matching unit.

Figure 5.6 and Figure 5.7 show the snapshots of the DCS map immediately following the addition of a new node, node \#120 into the network using the original and the modified neural network growing algorithms respectively. Figure 5.6 shows that the node that was improperly introduced in cycle \#115 in between the two spirals into a region of the network that represents no data (circle ' $A$ ' in Figure 5.6) gets rectified by neural network algorithm at the end of cycle \#120. Rectification here implies that the node that was introduced into a region of the network that represents no data gets absorbed into one of the two-spiral functions, i.e. into a region that represents data. However, note that it took the stochastic of the neural network training algorithm 5 adaptation cycles (120-115) to recover the network map back into a topology preserving mapping. Also, observe in Figure 5.6 that the original neural network growing algorithm introduces the new node, node \#120 into a region within the network (circled as 'B') between the two spirals that does not represent any data. Thereby, the map that is generated by the original neural network growing algorithm at the end of cycle \#120 (shown in Figure 5.6) is not a topology preserving mapping as the neighborhood relationship is not maintained. On the other hand, as shown in Figure 5.7, the modified growing algorithm introduces the new node (node \#120) into a network region that represents data in a topology preserving manner. 


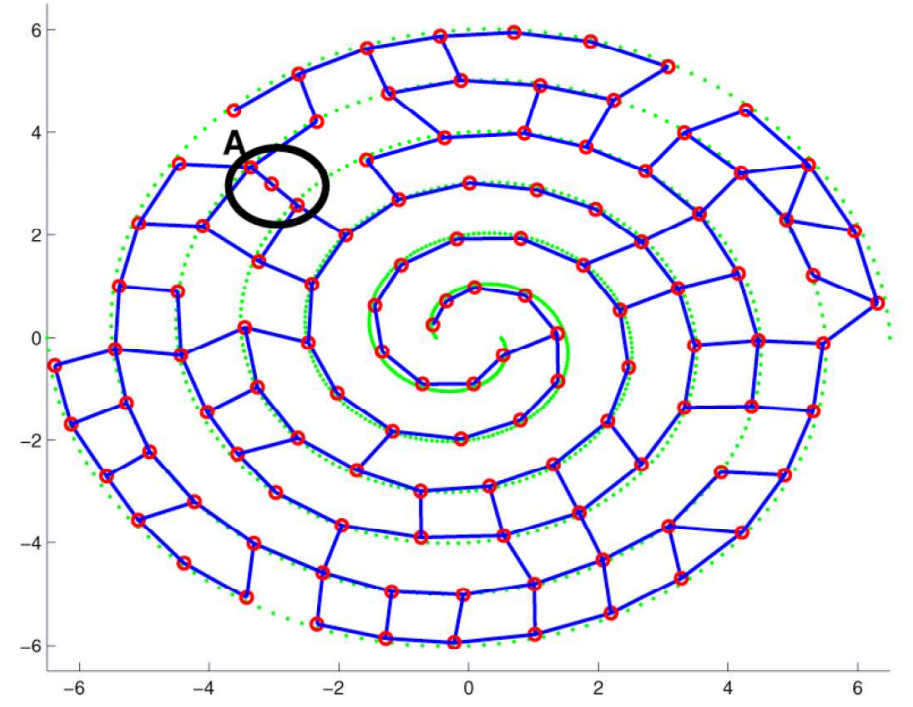

Figure 5.4 DCS using the original NN growing algorithm after 115 cycles

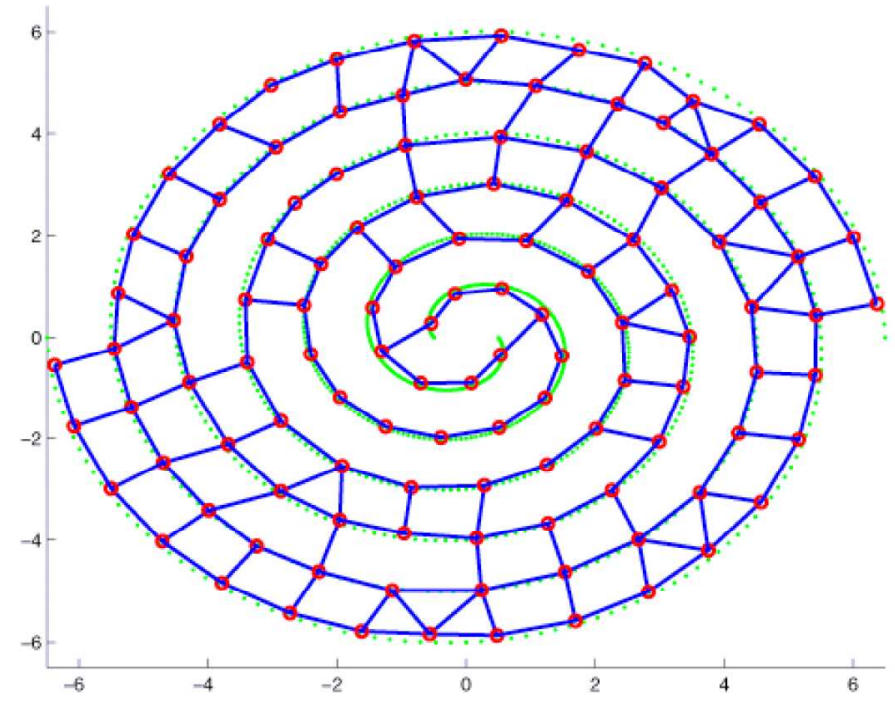

Figure 5.5 DCS using the modified NN growing algorithm after 115 cycles 


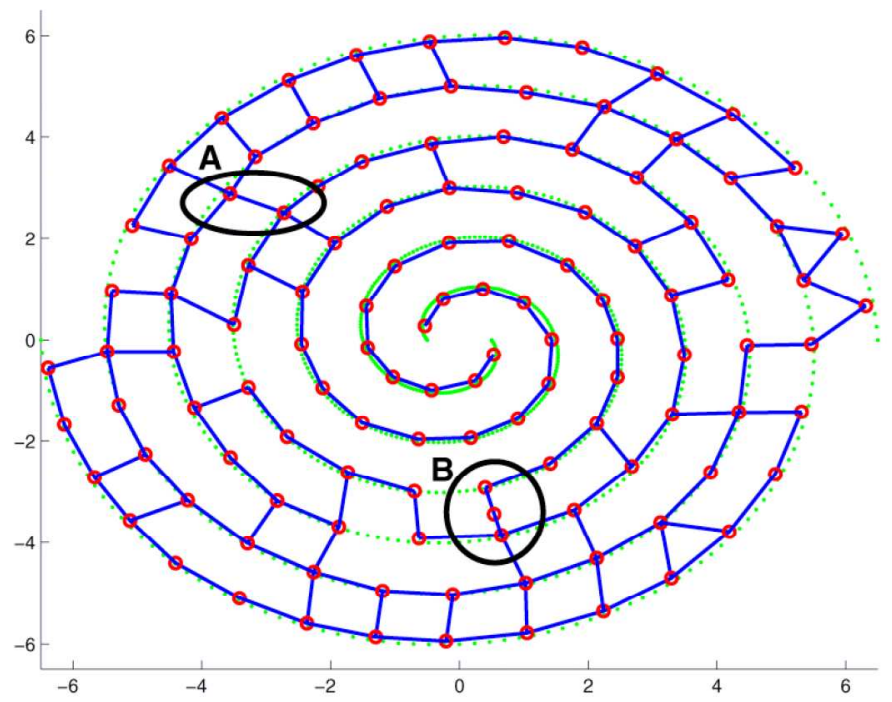

Figure 5.6 DCS using the original NN growing algorithm after 120 cycles

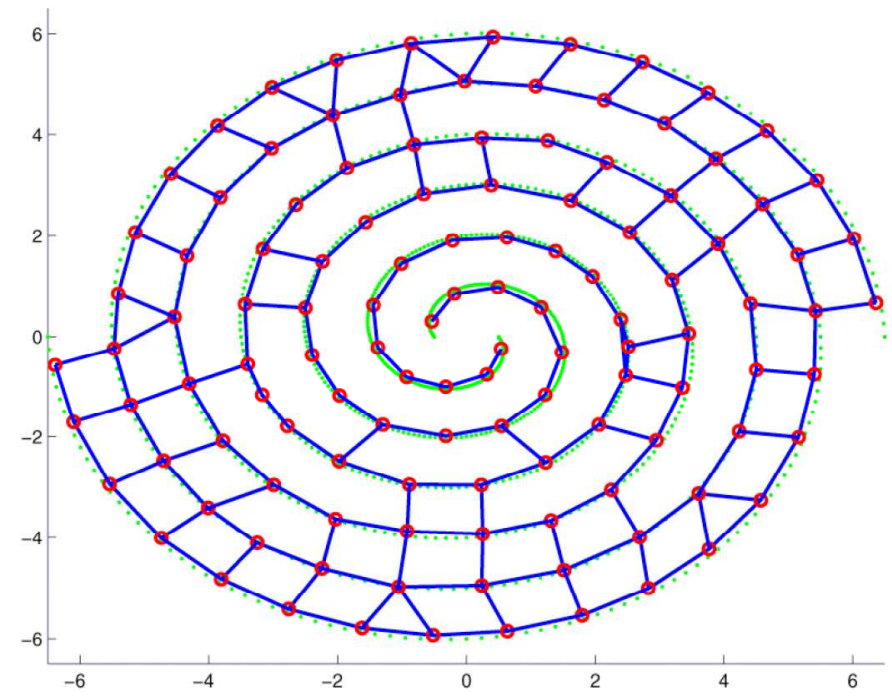

Figure 5.7 DCS using the modified NN growing algorithm after 120 cycles

Figure 5.8 and Figure 5.9 show the snapshots of the DCS SOM immediately following the addition of a new node, node \#128 into the network using the original and the modified neural network growing algorithms respectively. Figure 5.8 shows that before the neural network training algorithm rectifies the node that was improperly introduced in cycle $\# 115$ (circle ' $\mathrm{B}$ ' in Figure 5.8), the original neural network growing algorithm, introduces the new 
node (node \#128) between the two spirals into a region within the network that does not represent any data (circle ' $\mathrm{C}$ ' in Figure 5.8). This results in a mapping that is not topology preserving with respect to the twin-spiral data. As shown in Figure 5.9, the modified growing algorithm introduces the new node (node \#128) into the network region that represents data, resulting in a topology preserving mapping. This example clearly demonstrates that a proper selection of a neural network growing algorithm is necessary to ensure topology preservation in the SOM.

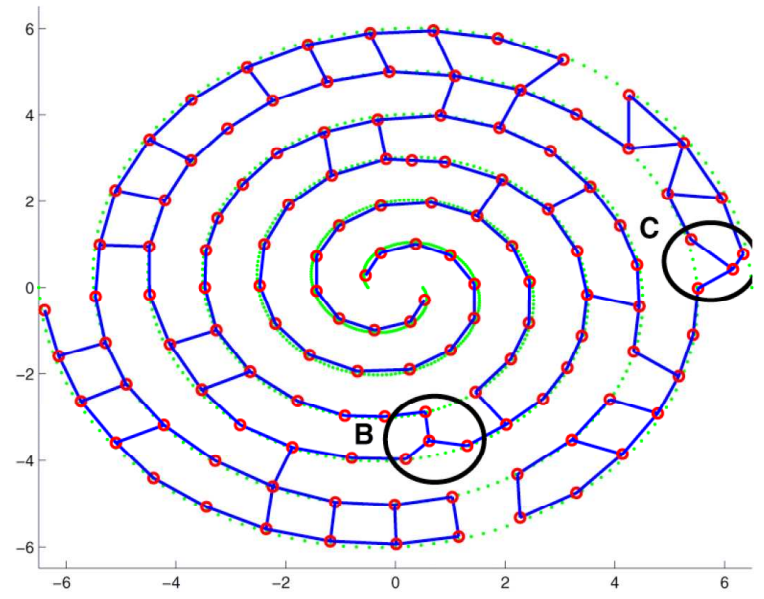

Figure 5.8 DCS using the original NN growing algorithm after 128 cycles

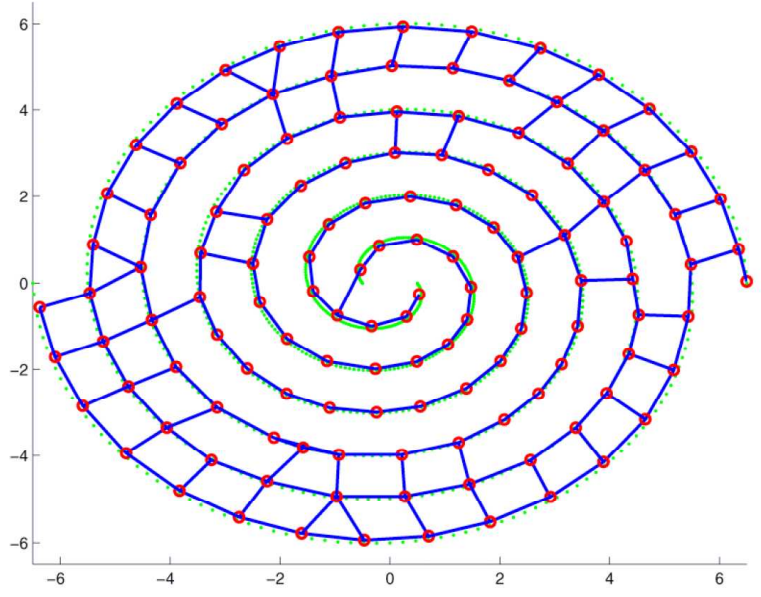

Figure 5.9 DCS using the modified NN growing algorithm after 128 cycles 
Figure 5.10 and Figure 5.11 show the snapshots of the DCS SOM immediately following the addition of a new node, node \#131 into the network using the original and the modified neural network growing algorithms respectively. Figure 5.10 shows that the nodes that were improperly introduced in cycles \#120 and \#128 in between the two spirals into regions of the network that represents no data (circle 'B' in Figure 5.6, circle 'C' in Figure 5.8) gets rectified by neural network algorithm at the end of cycle \#131. Note, however, that it took the stochastic of the neural network training algorithm 11 adaptation cycles $(131-120)$ to rectify the node that is improperly introduced in cycle \#120 (circle ' $\mathrm{B}$ ' in Figure 5.6), and 3 adaptation cycles (131-128) to rectify the node that is improperly introduced in cycle \#128 (circle ' $\mathrm{C}$ ' in Figure 5.8). Also, observe in Figure 5.10 that the original neural network growing algorithm introduces the new node, node \#131 into a region within the network (circle 'D' in Figure 5.10) between the two spirals that does not represent any data. Thereby, the map that is generated by the original neural network growing algorithm at the end of cycle \#131 (shown in Figure 5.10) is not a topology preserving mapping as the neighborhood relationship is not maintained. However, as shown in Figure 5.11, the modified growing algorithm introduces the new node (node \#131) into a network region that represents data in a topology preserving manner.

Figure 5.12 and Figure 5.13 show the snapshots of the DCS SOM immediately following the addition of a new node, node \#143 into the network using the original and the modified neural network growing algorithms respectively. Figure 5.12 shows that the node that was improperly introduced in cycle \#131 in between the two spirals into a region of the network that represents no data (circle ' $D$ ' in Figure 5.12) gets rectified by neural network algorithm at the end of cycle \#143. Note, however, that it takes the stochastic nature of the neural network training algorithm 12 adaptation cycles (143-131) to rectify the network map back into a topology preserving mapping. 


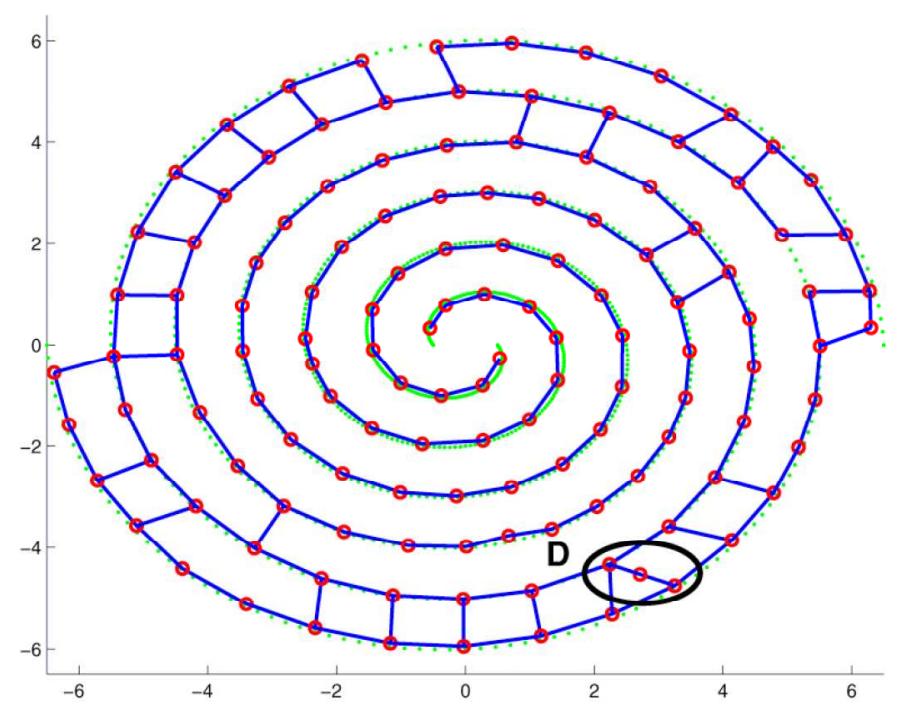

Figure 5.10 DCS using the original NN growing algorithm after 131 cycles

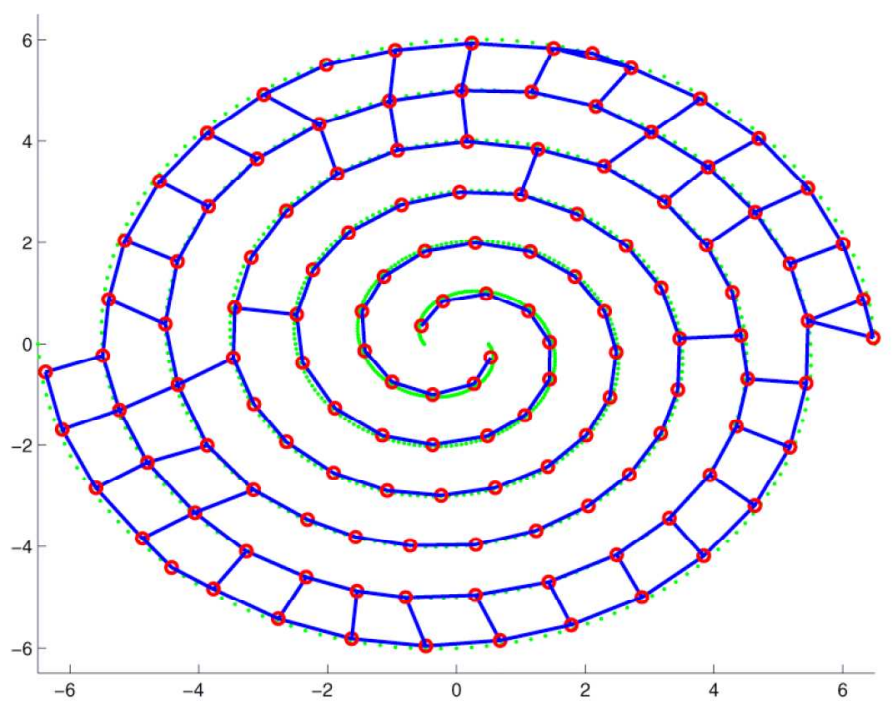

Figure 5.11 DCS using the modified NN growing algorithm after 131 cycles 
Also, observe in Figure 5.12 that the original neural network growing algorithm introduces the new node, node \#143 into a region within the network (circle 'E' in Figure 5.12) between the two spirals that does not represent any data. Thereby, the map that is generated by the original neural network growing algorithm at the end of cycle \#143 (shown in Figure 5.12) is not a topology preserving mapping as the neighborhood relationship is not maintained. As shown in Figure 5.13, the modified growing algorithm introduces the new node (node \#143) into a network region that represents data in a topology preserving manner.

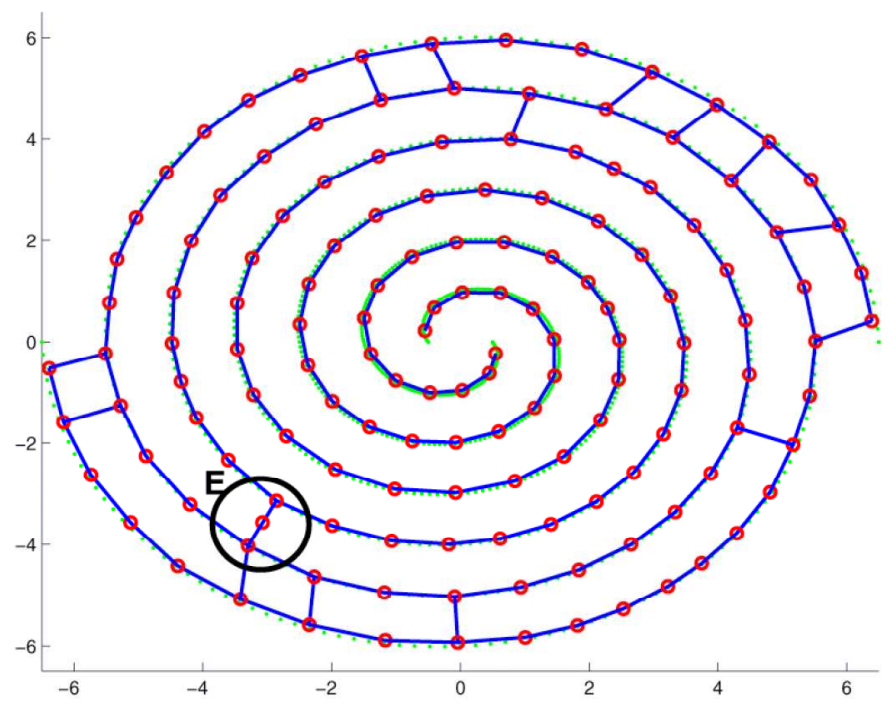

Figure 5.12 DCS using the original NN growing algorithm after 143 cycles 


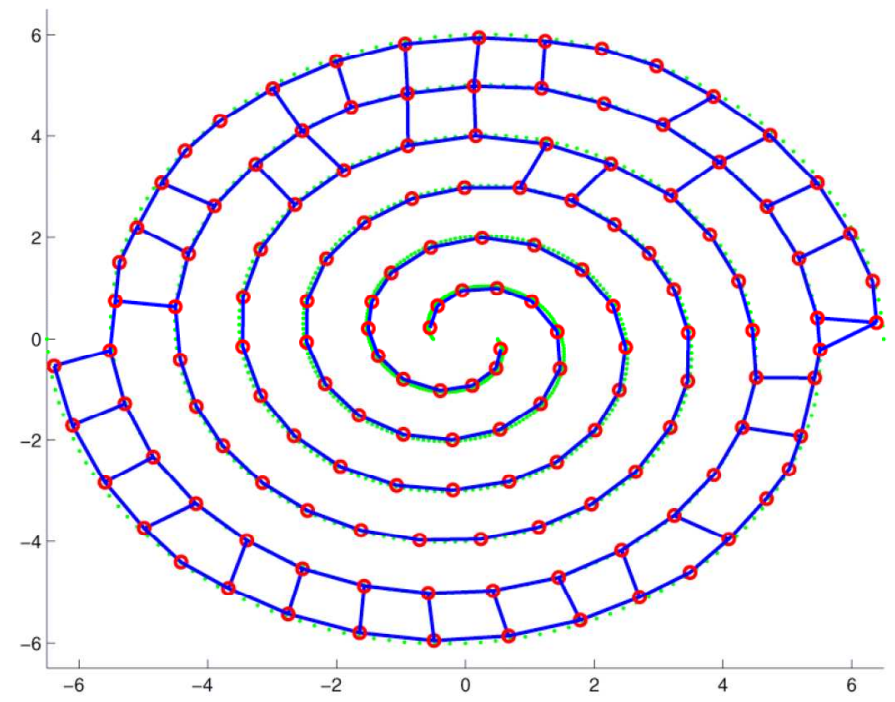

Figure 5.13 DCS using the modified NN growing algorithm after 143 cycles

Figure 5.14 and Figure 5.15 show the snapshots of the DCS SOM immediately following the addition of a new node, node \#152 into the network using the original and the modified neural network growing algorithms respectively. Figure 5.14 shows that even before the neural network training algorithm rectifies the node that was improperly introduced in cycle \#143 (circle ' $E$ ' in Figure 5.12), the original neural network growing algorithm introduces the new node (node \#152) between the two spirals into a region within the network that does not represent any data (circle ' $F$ ' in Figure 5.14). This results in a mapping that is not topology preserving with respect to the twin-spiral data. As shown in Figure 5.15, the modified growing algorithm introduces the new node (node \#152) into a network region that represents data, resulting in a topology preserving mapping. 


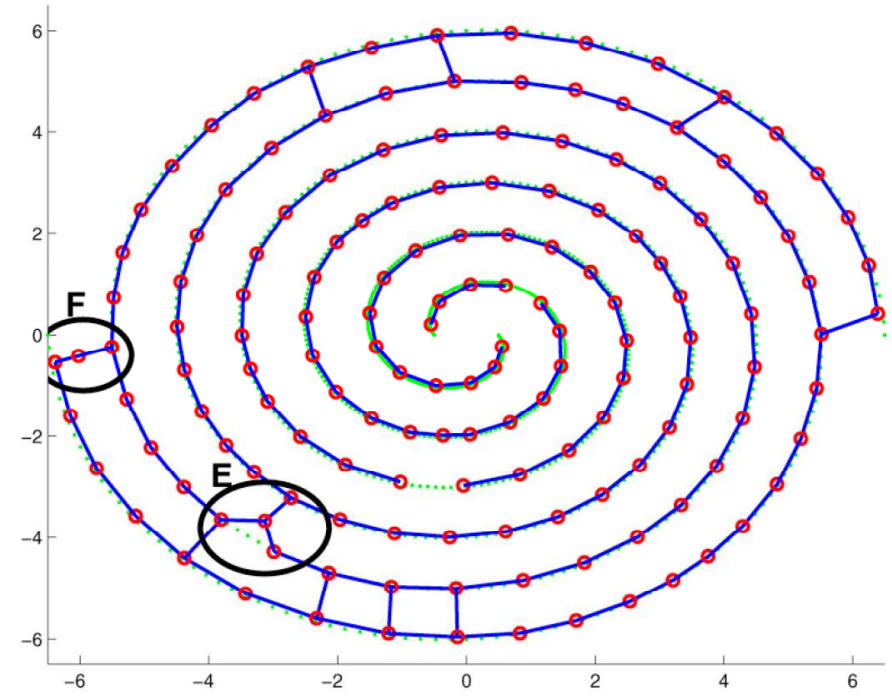

Figure 5.14 DCS using the original NN growing algorithm after 152 cycles

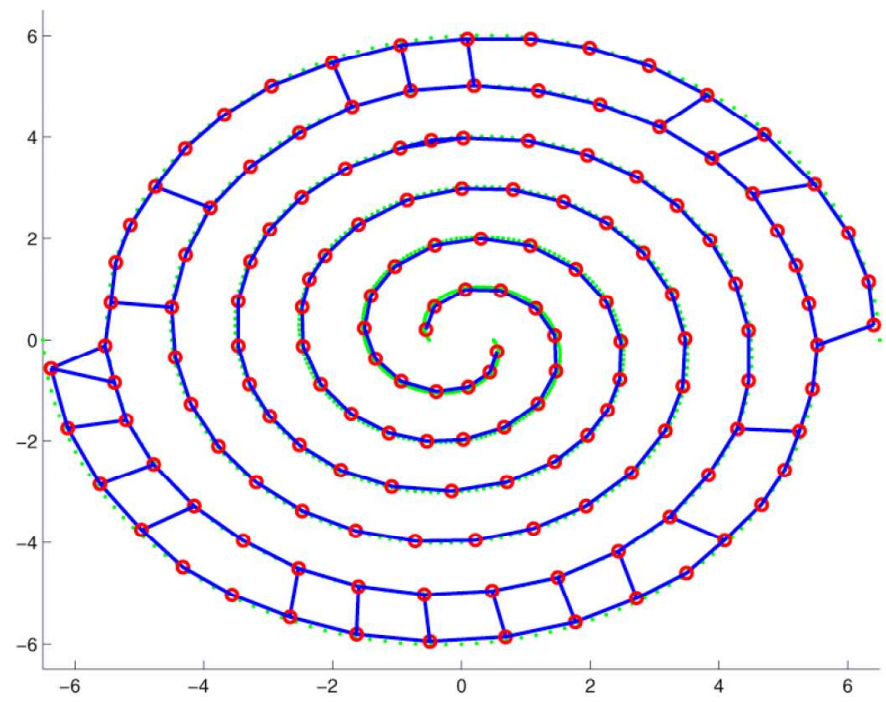

Figure 5.15 DCS using the modified NN growing algorithm after 152 cycles

Figure 5.16 and Figure 5.17 show the snapshots of the DCS SOM immediately following the addition of a new node, node \#172 into the network using the original and the modified neural network growing algorithms respectively. Figure 5.16 shows that the node that 
was improperly introduced in cycle \#143 in between the two spirals into a region of the network that represents no data (circle ' $E$ ' in Figure 5.12) gets rectified by neural network algorithm at the end of cycle \#172. However, note that it took the stochastic nature of the neural network training algorithm 29 adaptation cycles (172-143) to rectify the network map back into a topology preserving mapping. Figure 5.16 shows that even before the neural network training algorithm rectifies the node that was improperly introduced in cycle \#152 (circle ' $F$ ' in Figure 5.14), the original neural network growing algorithm introduces the new node (node \#172) between the two spirals into a region within the network that does not represent any data (circle 'G' in Figure 5.16). This results in a mapping that is not topology preserving with respect to the twin-spiral data. As shown in Figure 5.17, the modified growing algorithm introduces the new node (node \#172) into a network region that represents data, resulting in a topology preserving mapping.

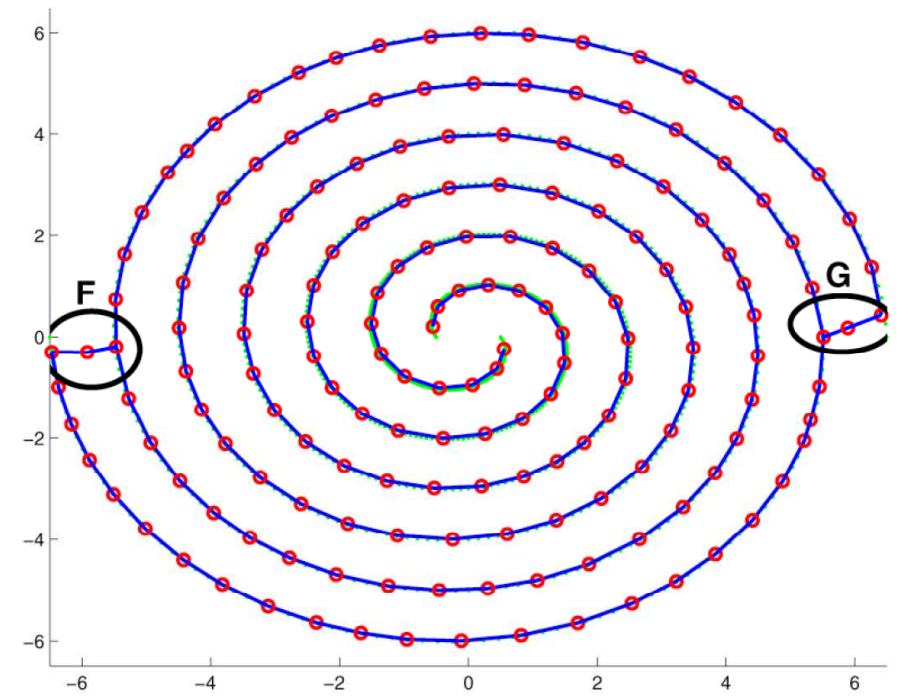

Figure 5.16 DCS using the original NN growing algorithm after 172 cycles 


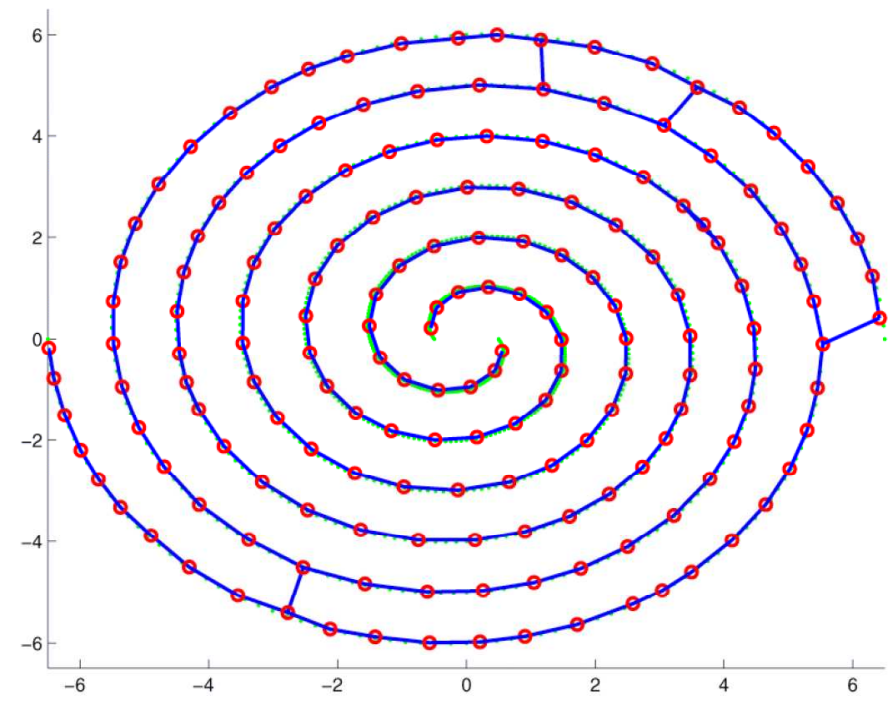

Figure 5.17 DCS using the modified NN growing algorithm after 172 cycles

Figure 5.18 and Figure 5.19 show the snapshots of the DCS SOM immediately following the addition of a new node, node \#180 into the network using the original and the modified neural network growing algorithms respectively. Figure 5.18 shows that the node that was improperly introduced in cycle \#172 in between the two spirals into a region of the network that represents no data (circle ' $G$ ' in Figure 5.16) gets rectified by neural network algorithm at the end of cycle \#180. Note, however, that it took the stochastic nature of the neural network training algorithm 8 adaptation cycles (180-172) to rectify the improperly introduced node. Also, observe from Figure 5.18 that the node that is improperly introduced in cycle \#152 in between the two spirals into a region of the network that represents no data (circle ' $F$ ' in Figure 5.14) is not rectified even after 28 adaptation cycles (180-152). Thereby, the map that is generated by the original neural network growing algorithm at the end of cycle \#180 (shown in Figure 5.18) is not a topology preserving mapping as the neighborhood relationship is not maintained. As shown in Figure 5.19, the modified growing algorithm introduces the new node (node \#180) into a network region that represents data in a topology preserving manner. 


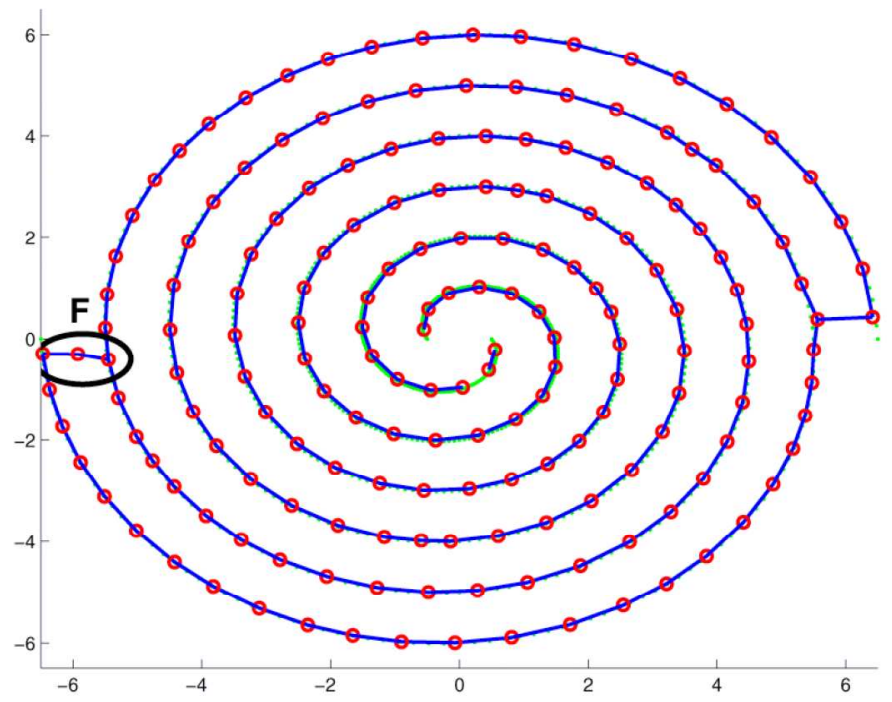

Figure 5.18 DCS using the original NN growing algorithm after 180 cycles

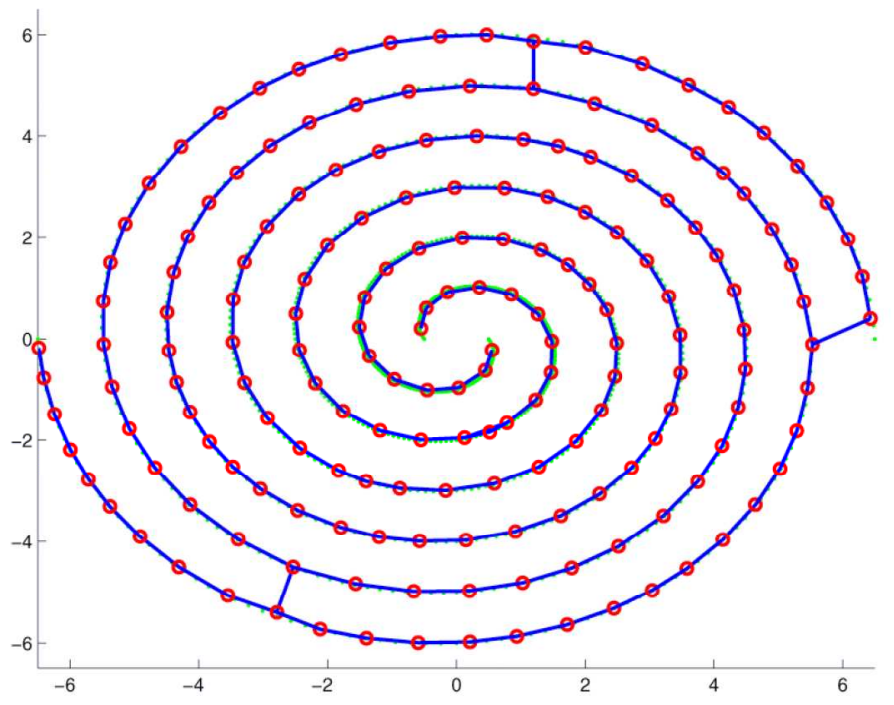

Figure 5.19 DCS using the modified NN growing algorithm after 180 cycles 
Figure 5.20 and Figure 5.21 show the snapshots of the DCS SOM immediately following the addition of a new node, node \#305 into the network using the original and the modified neural network growing algorithms respectively. Figure 5.20 shows that the node that was improperly introduced in cycle \#152 in between the two spirals into a region of the network that represents no data (circle ' $F$ ' in Figure 5.14) gets rectified by neural network algorithm at the end of cycle \#305. However, note that it took the stochastic nature of the neural network training algorithm 153 adaptation cycles (305-152) to rectify the improperly introduced node. Thereby, the map that is generated by the original neural network growing algorithm at the end of cycle \#305 (shown in Figure 5.20) is a topology preserving mapping. On the other hand, as shown in Figure 5.21, the modified growing algorithm introduces the new node (node \#305) into a network region that represents data, resulting in a topology preserving mapping. A noteworthy point here is that because the neural network training algorithm was unable to rectify the node that was improperly introduced in cycle \#152 (circle ' $F$ ' in Figure 5.14) until the end of cycle \#305, the mappings generated by the original growing algorithm from cycles \#152 to \#305 are all not topology preserving with respect to the twinspiral input data. 


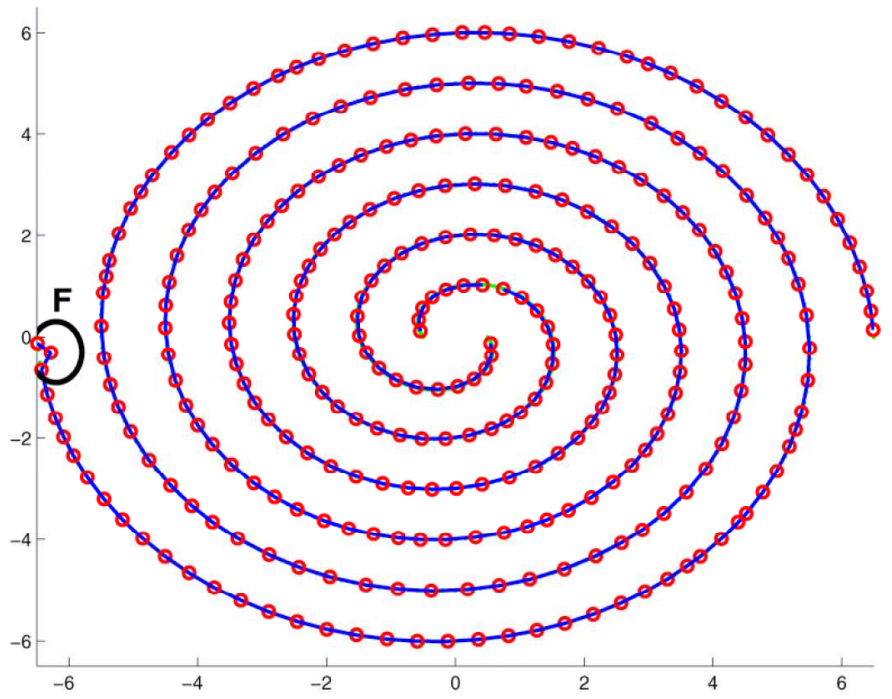

Figure 5.20 DCS using the original NN growing algorithm after 305 cycles

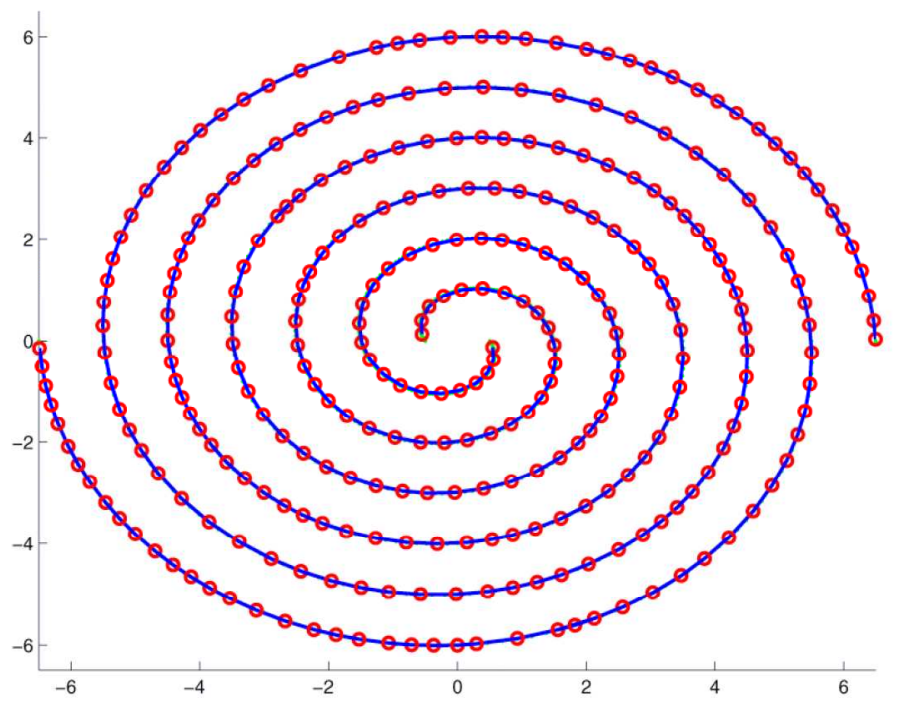

Figure 5.21 DCS using the modified NN growing algorithm after 305 cycles 
Table 5-1 summarizes the comparison results showing the number of improperly introduced nodes using the original and modified growing neural algorithms, and the number of cycles it requires for the neural network training algorithms to recover the map back into a topology preserving mapping. In the Table $5-1, X$ represents the number of nodes that were improperly introduced by the corresponding neural network growing algorithm into regions that represent no data. Recall that introduction of nodes into regions that represent no data results in a mapping that is not topology preserving with respect to the presented data. In the Table $5-1, Y$ represents the number of cycles required by the corresponding neural network growing algorithm to rectify the improperly introduced node.

Table 5-1 Comparison of original and modified NN growing algorithms

\begin{tabular}{l|l|c|c|c}
\hline $\begin{array}{l}\text { Learning } \\
\text { Cycle }\end{array}$ & \multicolumn{1}{|c|}{ Original Growing Algorithm } & \multicolumn{2}{c}{ Modified Growing Algorithm } \\
\hline 115 & \multicolumn{1}{|c|}{$\mathbf{X}$} & $\mathbf{X}$ & $\mathbf{Y}$ \\
120 & 1 ('A' in Figure 5.4) & -- & -- & -- \\
128 & 2 ('B', 'C' in Figure 5.6) & 5 for A & -- & -- \\
131 & 1 ('D' in Figure 5.10) & 11 for B, 3 for C & -- & -- \\
143 & 1 ('E' in Figure 5.12) & 12 for D & -- \\
152 & 2 ('E', 'F' in Figure 5.14) & -- & -- \\
172 & 2 ('F', 'G' in Figure 5.16) & 29 for E & -- & -- \\
180 & 1 ('F' in Figure 5.18) & 8 for G & -- & -- \\
305 & None (Figure 5.20) & 153 for F & -- & -- \\
\hline
\end{tabular}

As shown in the table there are many instances during the SOM learning using the original neural network growing algorithm where nodes are introduced into the network into regions that represent no data, thereby, leading to a mapping that is not topology preserving. 
However, in the case of the SOM learning using the modified neural network growing algorithm, nodes are always introduced into the network in regions that represent data, thereby, resulting in mappings that are always topology preserving.

The example demonstrates that addition of nodes into a SOM network region between nodes with higher accumulated local errors (resource values) does not always generate a mapping that is topology preserving with respect to the presented input data. The examples presented here also demonstrate that the proposed modifications enable the modified neural network growing algorithm to generate mappings that are always topology preserving with respect to the presented data. This is because the modifications in the neural network growing algorithm add nodes into the network by overlaying them over data patterns that are poorly represented by the network.

One of the most challenging and complex tasks of self-organization process is to ensure that the map generated by the neural network training algorithm is topology preserving with respect to the presented data. In this chapter, it is shown that current processes of dynamic allocation of network size and shape that are based on introducing nodes between nodes with high accumulated local errors need not necessarily generate a topology preserving mapping. While the twin-spiral data is used here as an example to demonstrate this idea, in practice, there exist a large class of data sets that exhibit similar problems. Based on the observed flaws with current processes of dynamic allocation of network size and shape, a modified neural network growing process is presented that utilizes the distribution of the input data along with the local error information. It is demonstrated, and evidently proved in the chapter using mathematical derivations that the presented neural network growing algorithm has a potential for overcoming the problems of inconsistency in topology preservation in growing SOMs. 


\section{Chapter}

\section{Generalization of NN Analysis}

Basing on the initial proposition of considering stability as a heuristic measure of reliability in system assurance, some stability results for NN were provided in Chapter 3 in the form of mathematical derivations. These results were based on the premise of considering NN adaptation as a dynamical system behavior, and were obtained using the formulations of stability according to Lyapunov's theory. In Chapter 4, the online stability-monitoring approach was presented as a runtime operational monitoring approach for detecting unstable NN behavior for situations where the mathematical stability results developed in Chapter 0 fail to apply. It should be realized that due to the system specific nature of Lyapunov stability analysis, the Lyapunov functions (and the stability results) provided in Chapter 3 and Chapter 4 are confined to the specific NN architecture, the DCS. The objective of this section is to generalize the developed NN analysis techniques for other neural network architectures such as feedforward, higher order, and linear neural networks. The discussion in this section is limited to the Sigma-Pi (S-Pi) and ADALINE neural networks. Recall that in the phase-I of the IFCS project, the DCS is the online learning neural network. Figure 6.1 shows the control architecture of the second generation of the IFCS system. In Phase II of the IFCS, the S-Pi and ADALINE neural networks serve (and replace the DCS) as the online learning neural networks (OLNN in Figure 6.1). The IFCS system in phase-II is based on a direct adaptive control scheme that incorporates neural networks [85].

The Sigma-Pi (S-Pi) neural network represents a feedforward neural network with a single hidden layer $[111,112,113]$. The S-Pi neural networks are such feedforward networks where each layer contains a higher-order term. Often the layers have summation units fed via weighted connections by intermediate product unit outcomes. The implementation details of the S-Pi neural network are provided in Section 6.1, followed by an extension of Lyapunov stability analysis for S-Pi neural network. 


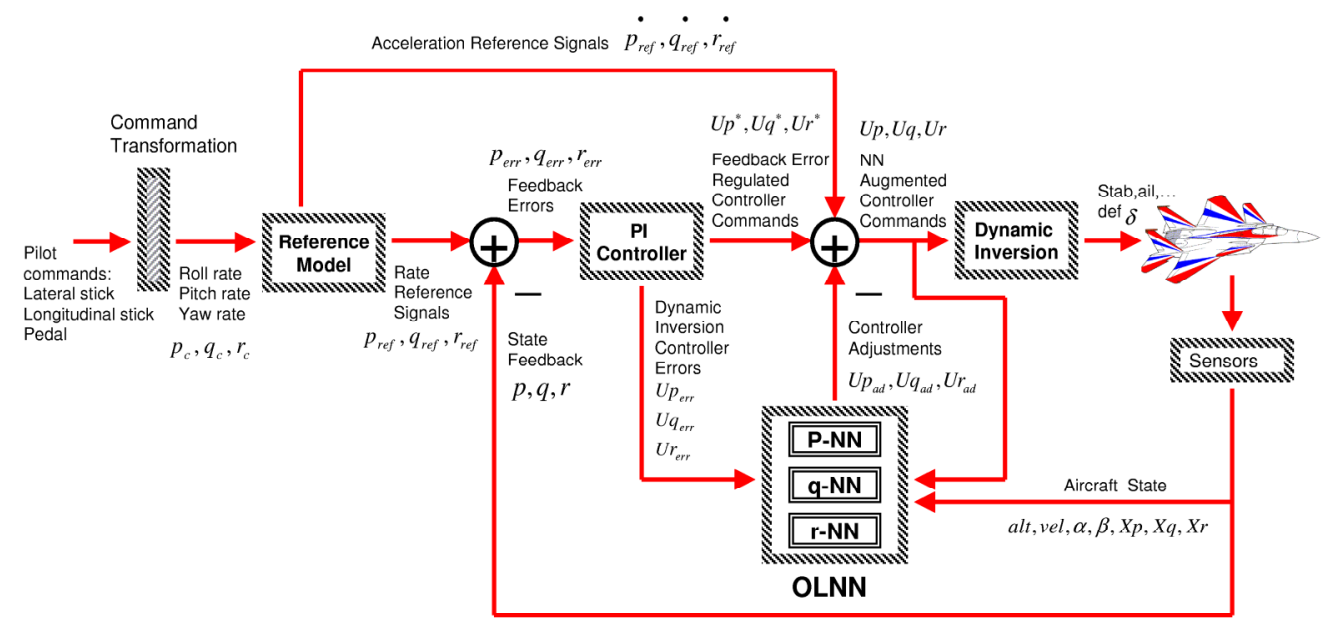

Figure 6.1 Control architecture of the Generation II of the IFCS

The ADALINE (adaptive linear) neural networks are an example of feedforward networks, which are named, so as all the data flows in one direction, from the input nodes to the output nodes $[114,115]$. The Adaline neural net, is essentially, a single-layer backpropagation neural network. The implementation details of the ADALINE neural network are provided in Section 6.2 followed by an extension of Lyapunov stability analysis for ADALINE neural network.

\subsection{Lyapunov Stability Analysis of Sigma-Pi NN}

A first-order connection (see Figure 6.2) characterizes most neural network architectures where a single (source) neuron is linked to a single (sink) neuron. However, it is realized that it is possible to link more than two neurons using a splicing function leading to the concept of higher-order connections as shown in Figure 6.3 [111]. 


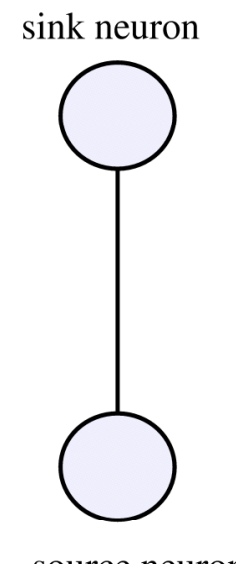

Figure 6.2 A first-order connection

Higher order connections link a set of (source) neurons to a single (sink) neuron, and are, in general, asymmetric. The connection order $(w)$ of the higher order connection is defined as the cardinality of the set of its source neurons. The higher-order connection that is shown in Figure 6.3 is of order 3. The splicing function (see Figure 6.3) combines the information from the source neurons before passing it to the sink neuron. Multiplication is the most commonly used splicing function in a higher-order connection. The set of source neurons in a higher order connection is usually located in a single layer [111].

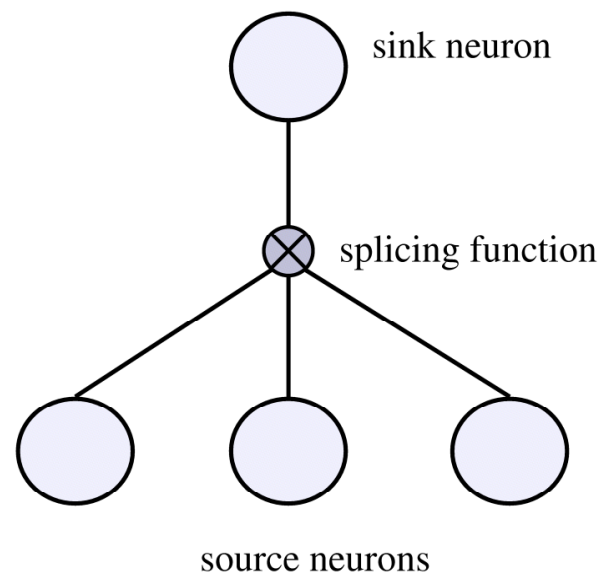

Figure 6.3 A higher-order connection 
In higher-order neural networks, the concept of higher-order connections is extended to the network level [111]. A higher-order neural network contains at least one higher-order connection. The order of the network $(\omega)$ is defined as the highest order connection.

$$
\Omega=\max _{\omega} \omega_{\omega}
$$

The history of high-order neural networks includes the work of Poggio [116] who coined the term 'high order', and Feldman and Ballard who used multiplication as splicing function and the connections are named conjunctive connections [117]. An important and fundamental contribution to the area of high-order neural networks, which has given rise to their wider dissemination, is the work by Lee et al [118].

The main advantage of higher-order neural networks is that they are capable of extracting higher-order information from the input data [111]. The higher order neural networks (HONN) have been developed with the intention to enhance the nonlinear descriptive capacity of the feed-forward multiplayer Perceptron neural networks (MLP) [111, 112]. In HONN, this is achieved by means of increasing the nonlinear descriptive capability of the individual neurons. A HONN builds multivariate high-order polynomial models,

$$
P(x)=W_{0}+\sum_{i} W_{i} x_{i}+\sum_{j} \sum_{i} W_{i} x_{i} x_{j}+\mathrm{K}
$$

The Equation (6.2) can be written concisely as,

$$
P(x)=W_{0}+\sum_{i=1}^{m} W_{i} \prod_{j=1}^{d} x_{j}^{r}
$$

In (6.2) and (6.3), $W_{i}$ represent the neural network weights, $M$ is the maximal number of monomials, $x_{i}, x_{j}$ are the features from the input vector, and $r=\{0,1,2, \mathrm{~K}\}$ is the powers with which the feature $j$ participates in the monomial $i$. Figure 6.4 shows the network architecture of a fully connected higher order neural network [111]. 


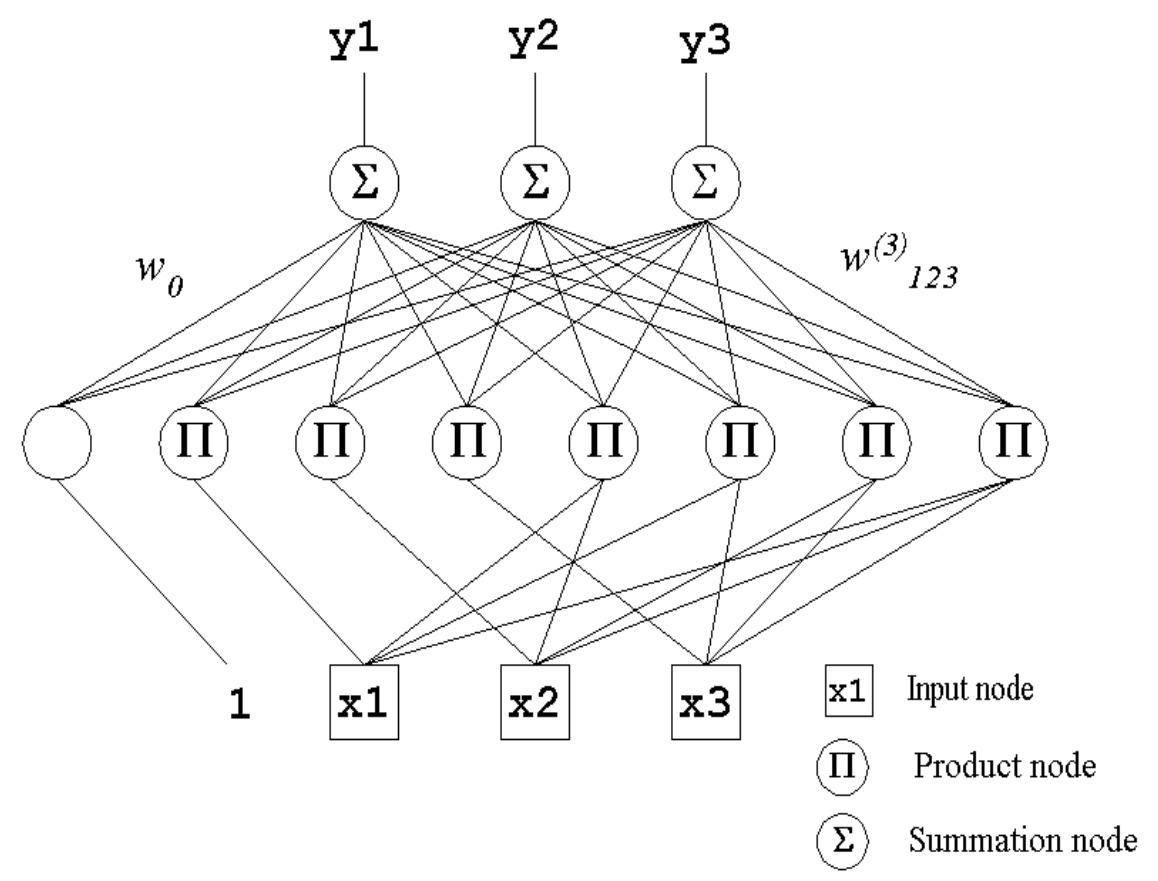

Figure 6.4 A Fully Connected Higher Order Neural Network

The nonlinearity of the functions that these higher order neural networks model is constructed using a cascade of several layers having summation (sigma) as well as product (pi) units. In the neural network literature, such networks are referred to as Sigma-Pi (S-Pi) neural networks. The S-Pi neural networks are such feedforward networks where each layer contains higher-order terms and the splicing function is multiplication. Often the layers have summation units fed via weighted connections by intermediate product unit outcomes.

In the S-Pi neural net, the sigma unit compute the sum of weighted inputs $P_{j}$ from the lower $j$ layer:

$$
s=W_{i} P_{j}
$$

The pi units compute the product of weighted inputs $x_{i}$ from the lower $i$ layer:

$$
P_{j}=\prod_{i} W_{i} x_{i}
$$


The output of the units is passed through the sigmoid activation function.

$$
O=F(s)=\frac{1}{1+e^{-s}}
$$

Figure 6.5 shows the network architecture of a third-order S-Pi neural network. The SPi neural nets are sparsely connected higher order nets with the polynomial order (network topology) restricted to a configuration sufficient in order to achieve the desired degree of accuracy. This restriction in the network topology is based on priori knowledge about the given function or application [111].

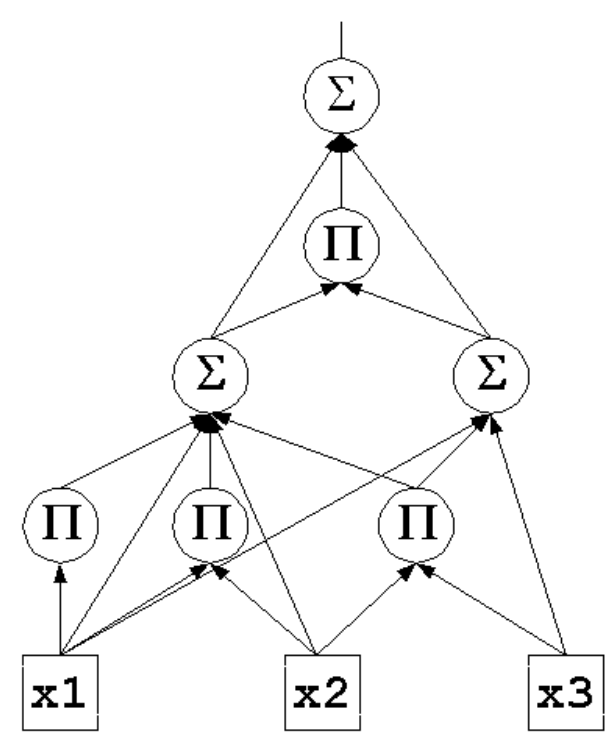

Figure 6.5 A Third Order Sigma-Pi Neural Network

In Phase II of the IFCS project, the S-Pi neural network serves as the online learning neural network and provides control adjustments to the flight control system (Figure 6.1). The S-Pi neural networks work with the error handling system in the IFCS to provide additional control adjustments to enable the IFCS system reduce the error more quickly [85, 91]. The SPi neural networks learn patterns within the error and produce biases to offset the errors. The learning rule followed in the S-Pi neural network for the IFCS is the error-modified, gradient descent learning algorithm [91]. The learning algorithm is shown in Figure 6.6. 


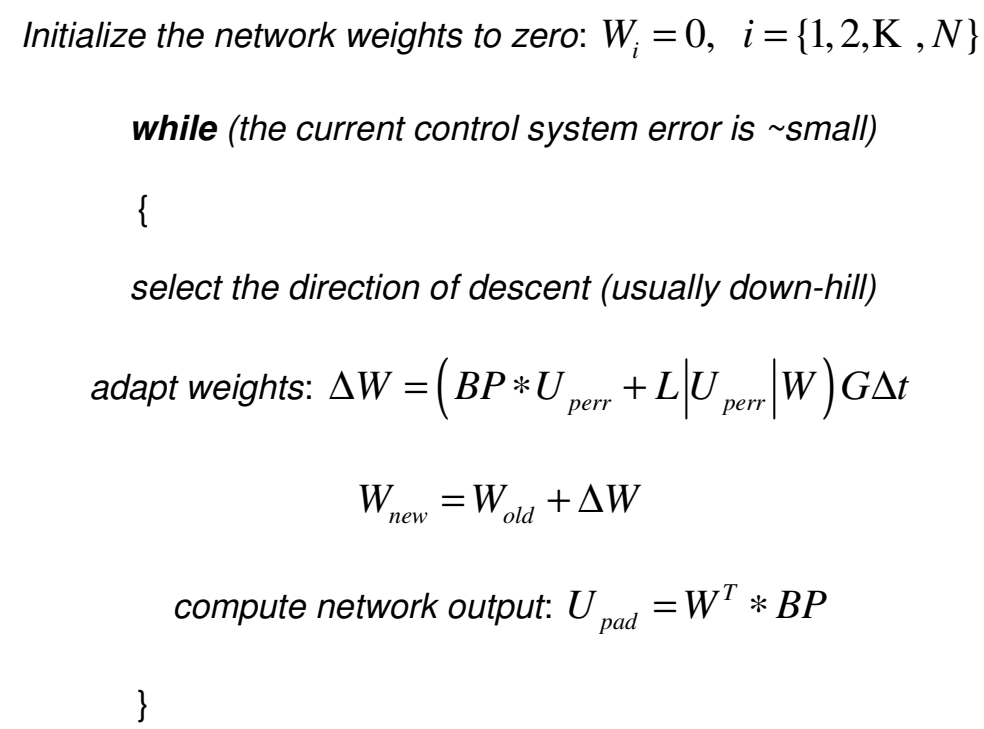

Figure 6.6 E-modified Neural Network Learning Algorithm

In Figure 6.6, $\Delta W$ represents the change in the weight adaptation, $B P$ represents the nested Kronecker product, Uperr represents the roll acceleration error from the control system, $G$ and $L$ are user-specified gains. The output of the neural network of Figure 6.6 is computed necessarily as

$$
U p a d=W^{T}(B P)
$$

A Lyapunov function candidate for the S-Pi neural network is the first derivative of the error with respect to the weights which is written as

$$
V=\left\|\frac{\partial E}{\partial W}\right\|
$$

In (6.8), $E=O_{d e s}-O_{a c t}$ represents the network error, the difference between the desired (or expected) network output and the actual network output. The Lyapunov function in (6.8) measures the change in the network error with respect to the change in the network weights. It should be emphasized here that on-line stability monitors are still needed in order to understand the online adaptation behavior of the network. 
An $N^{\text {th }}$ order S-Pi neural network may consist of $0,1^{s t}, 2^{\text {nd }}, \mathrm{K}, N^{\text {th }}$ order networks, but the highest network order (or cardinality of the inputs) is $N$. An online stability monitoring system for the online learning S-Pi neural network can consist of stability monitors that monitor the behavior of all orders of the neural network. For instance, an $N^{\text {th }}$ order S-Pi neural network consisting of $0,1,2, \mathrm{~K}, N$ order weights could be monitored using $N+1$ stability monitors such that each monitor analyzing an individual order weight adaptation terms. Let $\Delta W_{i 1}, \Delta W_{i 2}, \mathrm{~K}$ represent the weight adjustments of the $i^{t h}$ order weight terms. The formulation of a monitoring system for an $N^{\text {th }}$ order neural network consisting of $0,1,2, \mathrm{~K}, N$ order weight terms is as follows.

$$
\begin{aligned}
& \text { Monitor } 1=\operatorname{sum}\left\{\Delta W_{01}, \Delta W_{02}, \mathrm{~K}\right\} \\
& \text { Monitor } 2=\operatorname{sum}\left\{\Delta W_{11}, \Delta W_{12}, \mathrm{~K}\right\} \\
& \mathrm{M} \\
& \text { Monitor } \mathrm{i}=\operatorname{sum}\left\{\Delta W_{(i-1) 1}, \Delta W_{(i-1) 2}, \mathrm{~K}\right\} \\
& \mathrm{M} \\
& \text { Monitor }(N+1)=\operatorname{sum}\left\{\Delta W_{N 1}, \Delta W_{N 2}, \mathrm{~K}\right\}
\end{aligned}
$$

Figure 6.7 shows the Matlab-Simulink implementation of the roll-axis, on-line learning S-Pi neural network. In Figure 6.7, the $U \_p \_e r r \_i n$ block shown in blue represents the control system error Uperr, and CP1, CP2, and CP3 represent the roll ( $\mathrm{p}$-channel) neural network inputs. Figure 6.8 shows the subsystem details of the roll-axis, neural network block of Figure 6.7. In Figure 6.8, the term $P \_n n \_a d \_r s p 2$ represents the roll ( $p$-channel) neural network output (Upad in (6.7)), and the NN Monitor block contains the stability monitoring system implementation given in (6.9). Figure 6.9 shows the implementation of the online learning, S-Pi neural network weight update algorithm that is given in Figure 6.6. The input to the S-Pi neural network consists of parameters such as aircraft velocity, altitude, $\alpha$ : angle of attack, etc. The parameters are encapsulated by the neural network input terms, CP1, CP2, and CP3. The 
CP1 term consists of CP1 bias (constant), normalized aircraft velocity, square of the normalized velocity, and the normalized altitude.

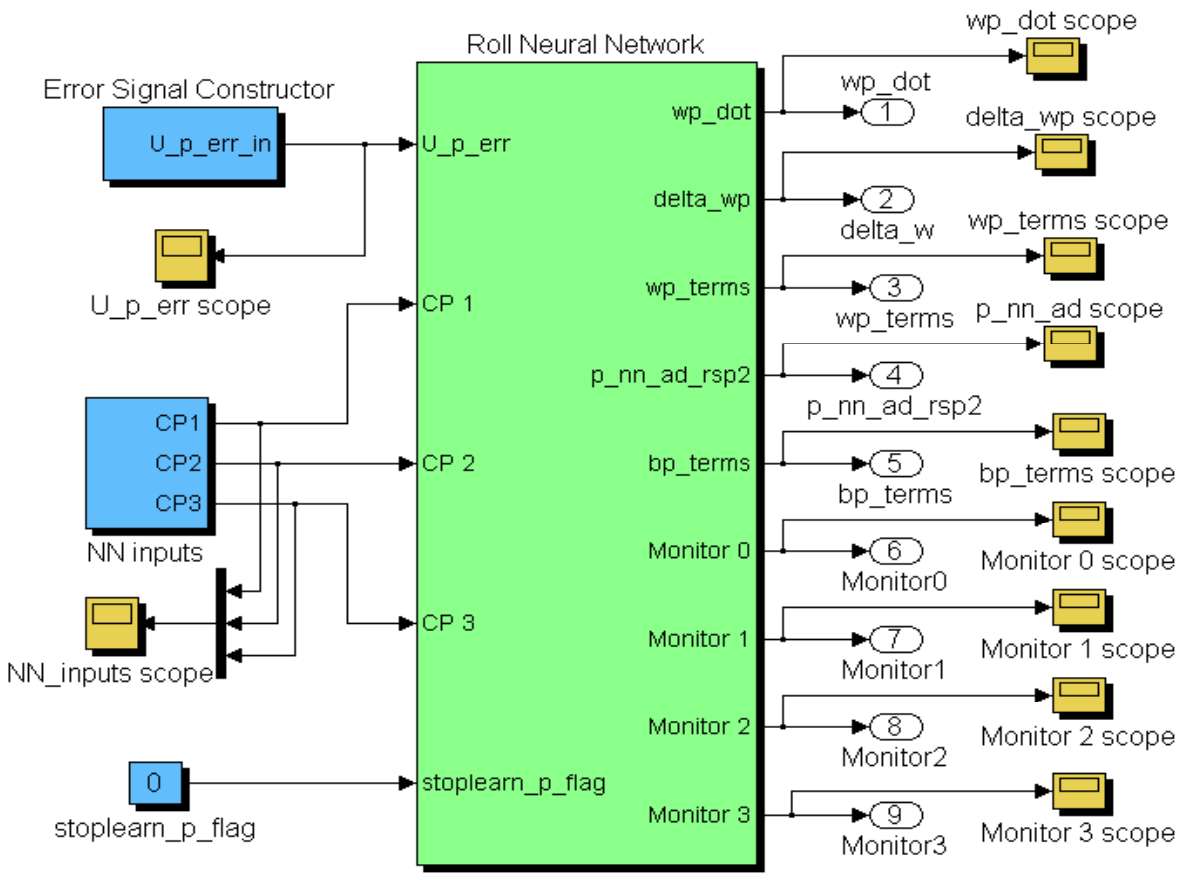

Figure 6.7 Matlab Simulink implementation of online learning S-Pi NN

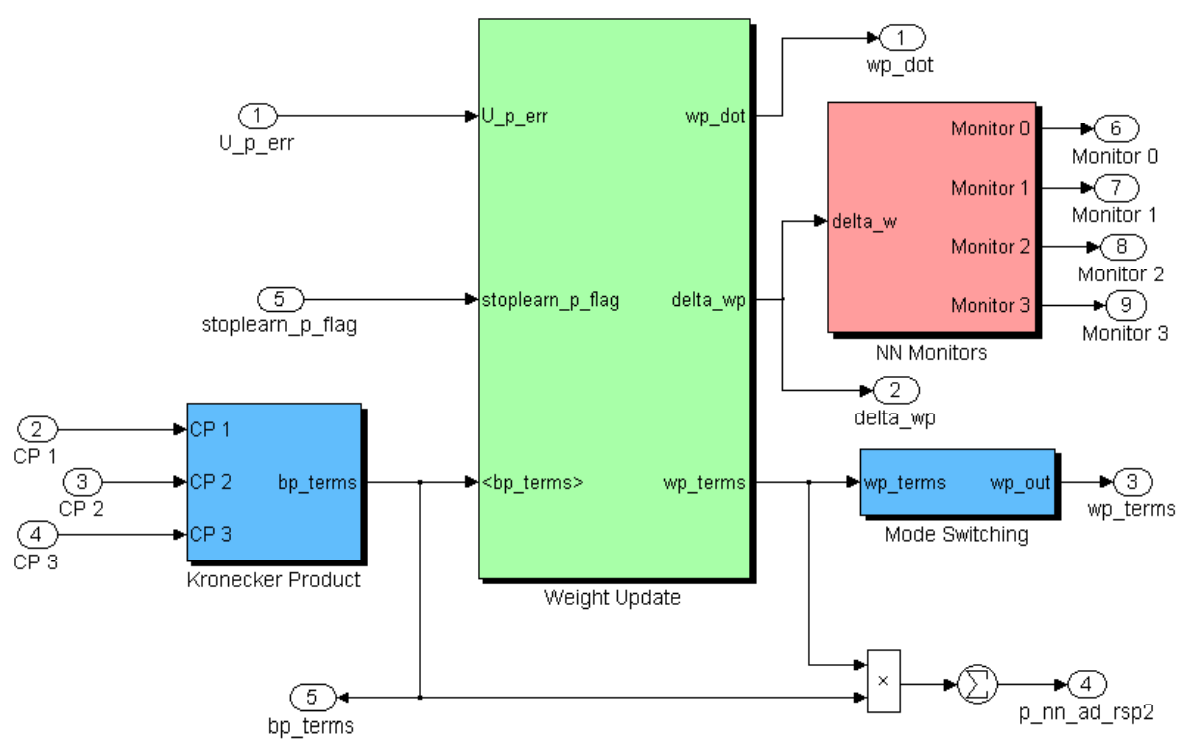

Figure 6.8 Matlab Simulink subsystem of the Roll S-Pi NN block of Figure 6.7 


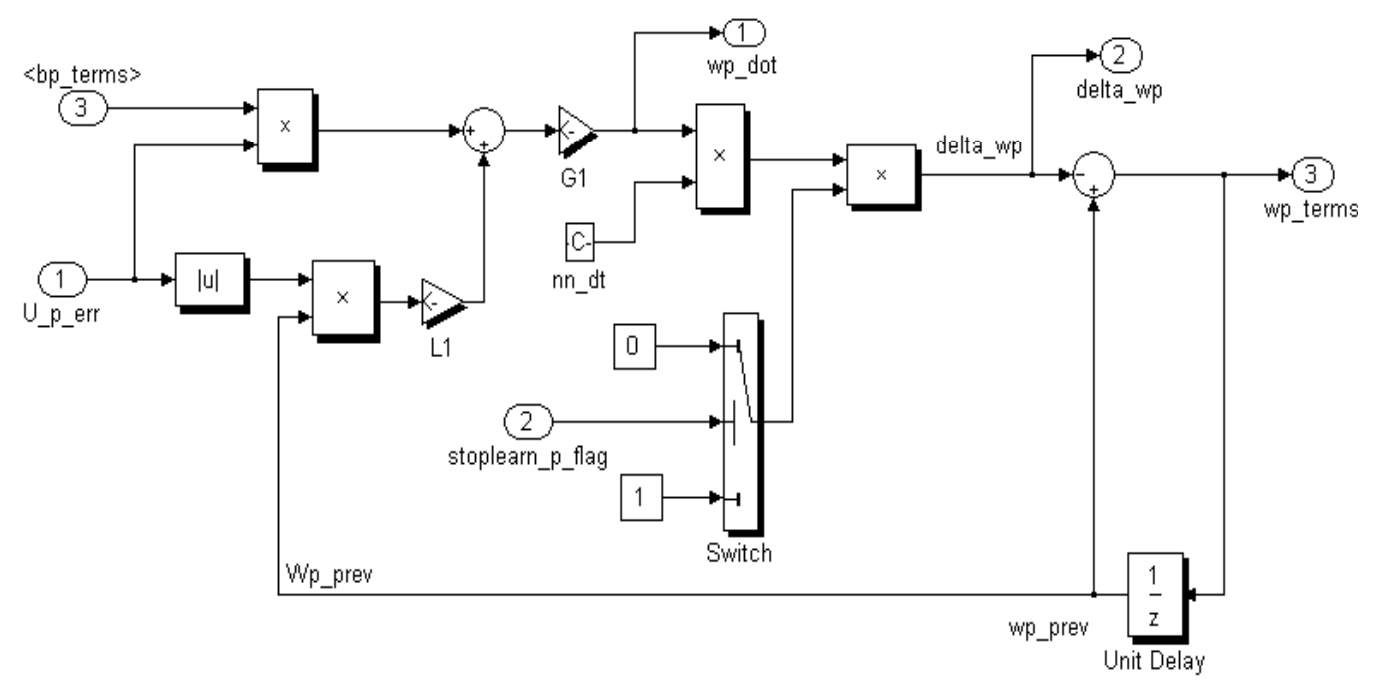

Figure 6.9 Matlab Simulink subsystem of the Weight Update block of Figure 6.8

$$
\mathrm{CP} 1=\left[\begin{array}{l}
\mathrm{CP} 1 \text { bias } \\
(\mathrm{Vt}-300) /(800-300) \\
{\left[\begin{array}{l}
(\mathrm{Vt}-300) /(800-300) \\
{[}
\end{array}\right]^{2}} \\
(\text { Alt }-30,000) /(80,000)
\end{array}\right]
$$

In (6.10), Vt is the velocity of the aircraft, and Alt is the altitude of the aircraft. Data is collected from the phase II implementation of the IFCS flight simulator for 30 seconds of flight with a sampling frequency of $80 \mathrm{~Hz}(20 \mathrm{~Hz}$ for phase I). Figure 6.10 shows the CP1 bias term that is set to a constant value of 2. Figure 6.11 shows a plot of the normalized CP1 velocity term. Figure 6.12 shows a plot of the square of normalized CP1 velocity term. Figure 6.13 shows a plot of the normalized CP1 altitude term. 


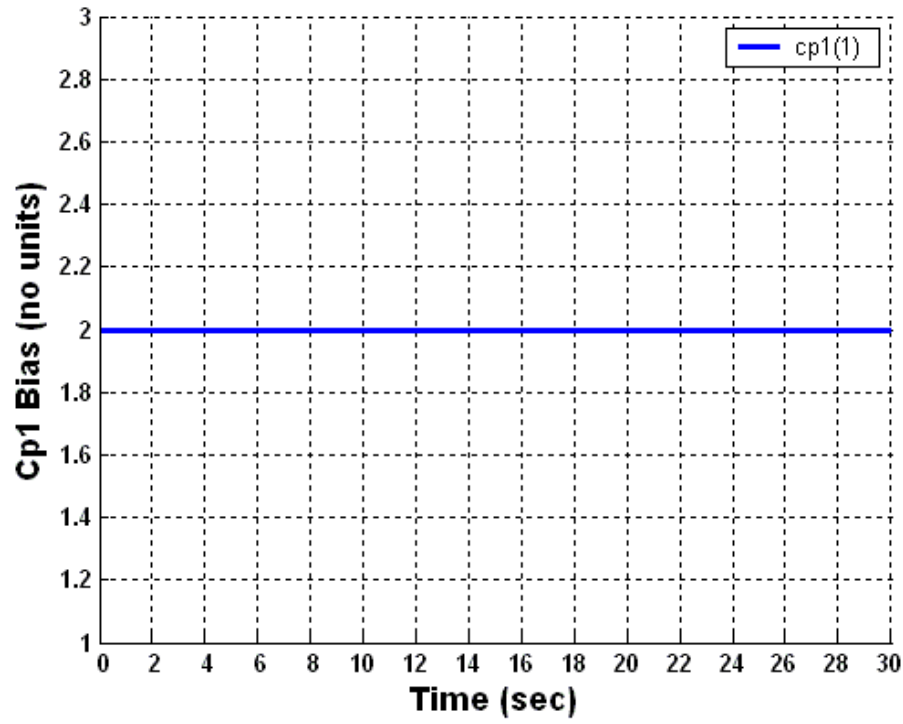

Figure $6.10 \mathrm{CP} 1$ bias term

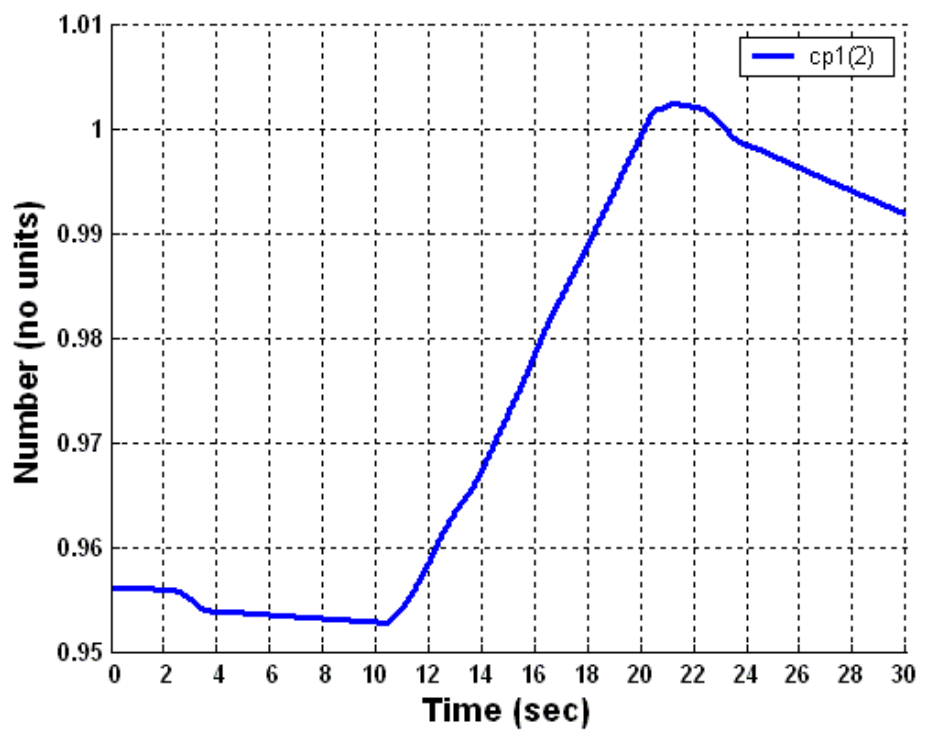

Figure 6.11 CP1 term: Normalized aircraft velocity 


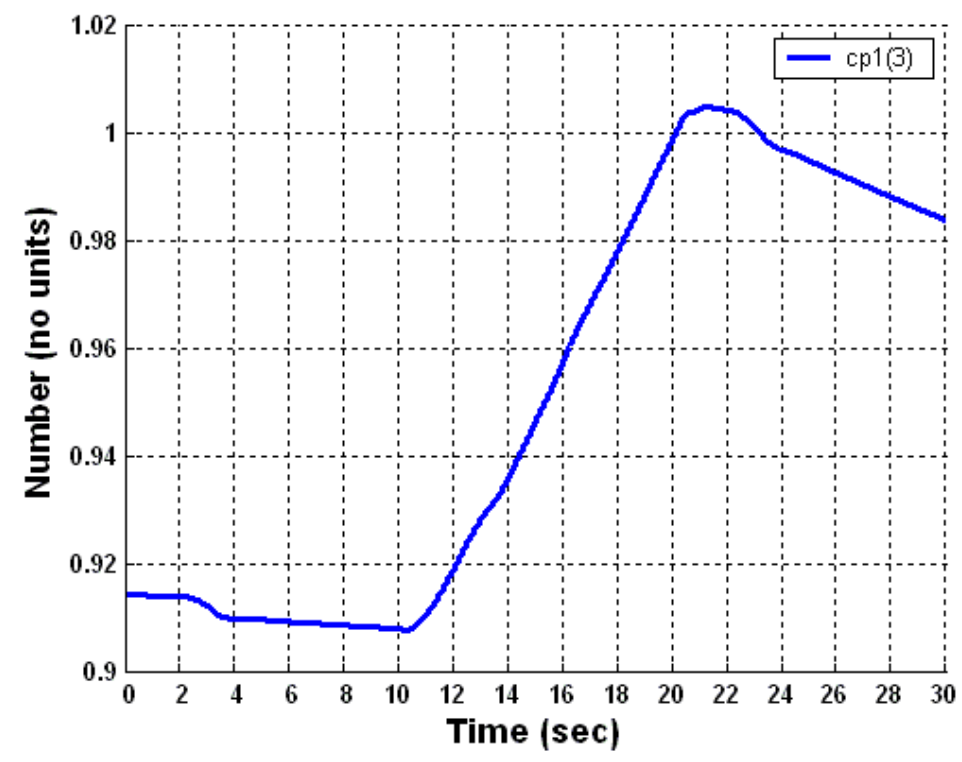

Figure 6.12 CP1 term: Square of normalized aircraft velocity

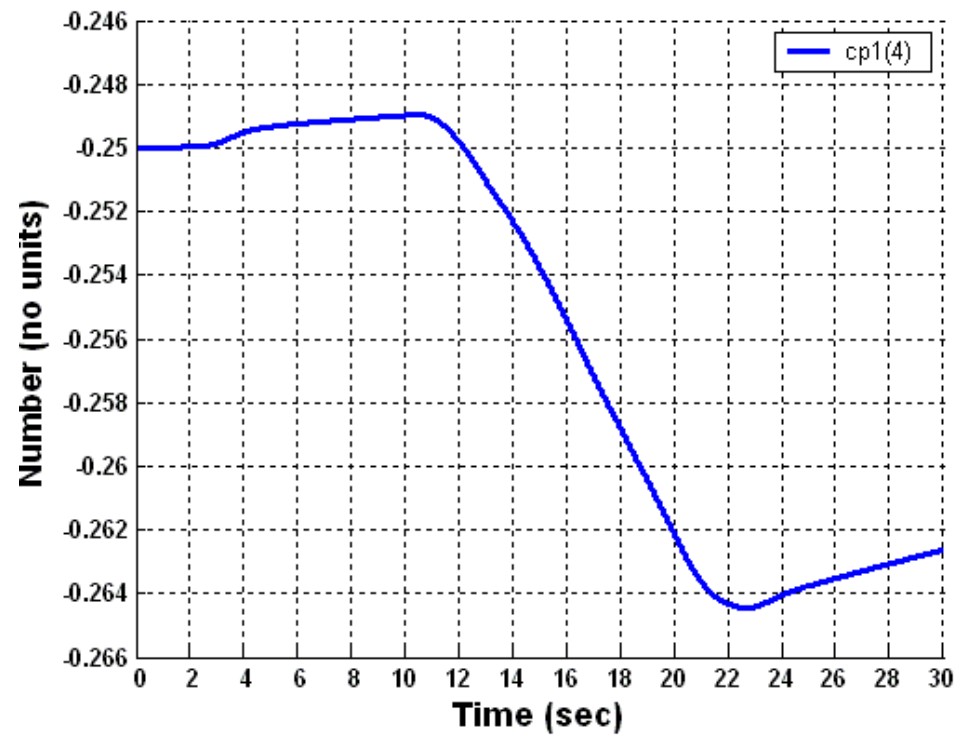

Figure $6.13 \mathrm{CP} 1$ term: Normalized aircraft altitude 
The CP2 term consists of CP2 bias (constant), control system error term, pitch body signal $(\mathrm{rad} / \mathrm{sec})$, yaw body signal $(\mathrm{rad} / \mathrm{sec})$, and reference model roll acceleration term $\left(\mathrm{rad} / \mathrm{sec}^{2}\right)$

$$
\mathrm{CP} 2=\left[\begin{array}{c}
\mathrm{CP} 2 \text { bias } \\
\left(1-e^{(\text {Up+Upad })}\right) \\
\text { Pitch Body Signal } \\
\text { Yaw Body Signal } \\
\text { Reference Model Roll Acceleration }
\end{array}\right]
$$

In (6.11), $U p$ is a control system error term, and Upad is the roll (p-channel) neural network output. The following plots are generated using the 30 seconds of flight data collected from the phase II implementation of the IFCS flight simulator. Figure 6.14 shows the CP2 bias term that is set to a constant value of 1.25. Figure 6.15 shows a plot of the second term of CP2, the control system term (see (6.11)). Figure 6.16 shows a plot of the pitch body signal. Figure 6.17 shows a plot of the yaw body signal. Figure 6.18 shows a plot of the reference model roll acceleration term.

The CP3 term consists of CP3 bias (constant), angle of attack, and sideslip.

$$
\mathrm{CP} 3=\left[\begin{array}{l}
\mathrm{CP} 3 \text { bias } \\
\alpha \\
\beta
\end{array}\right]
$$




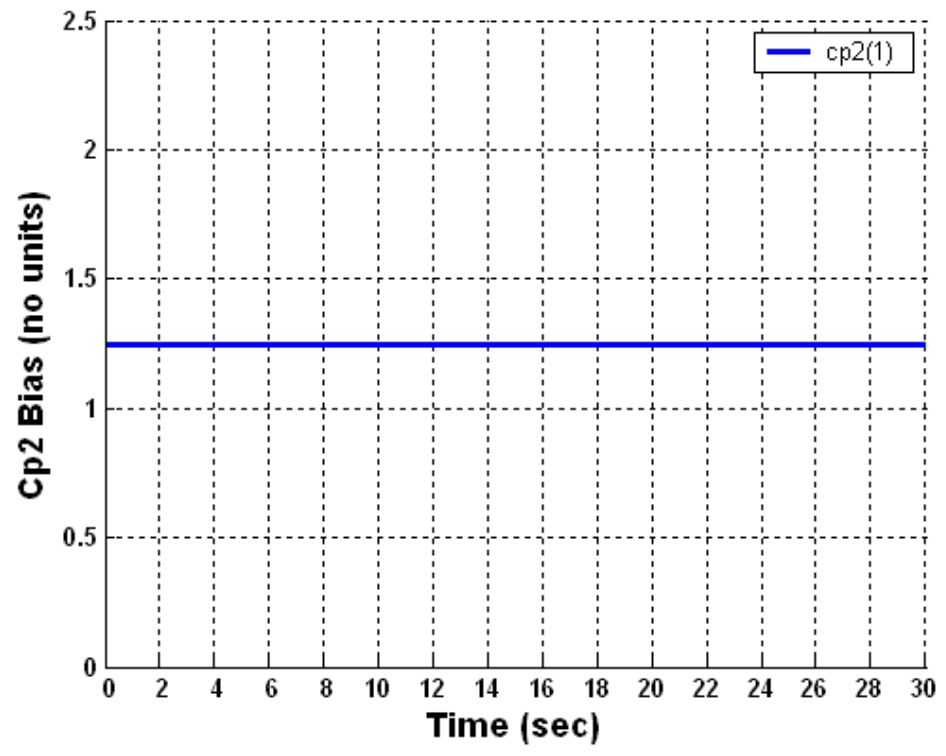

Figure 6.14 CP2 bias term

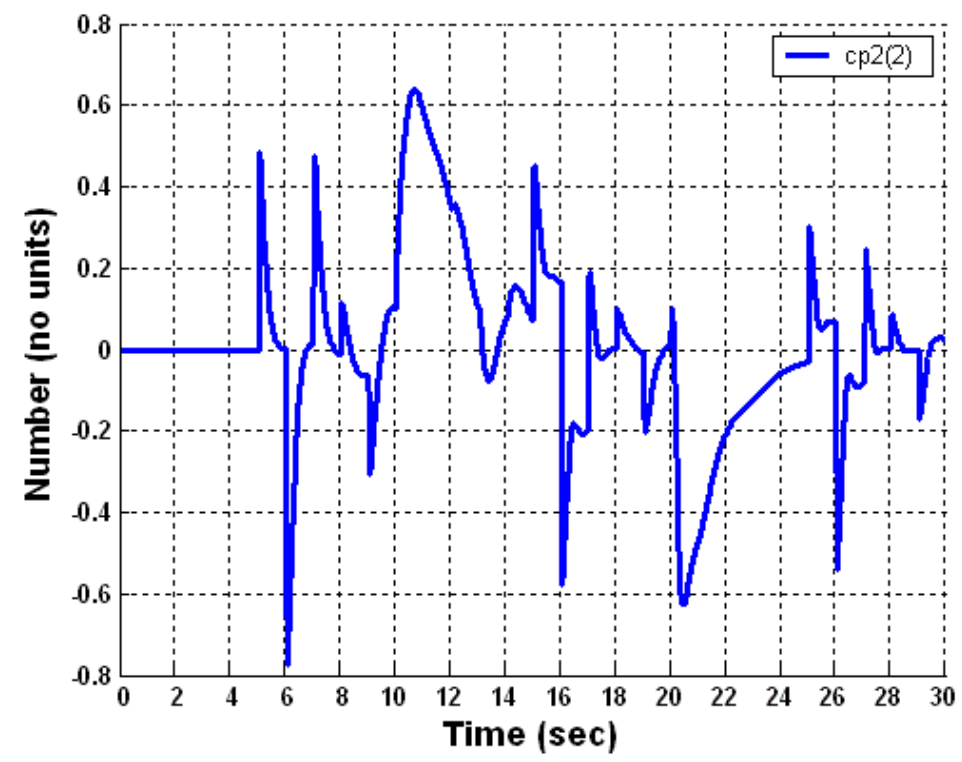

Figure 6.15 CP2 term: squash(Up+Upad) 


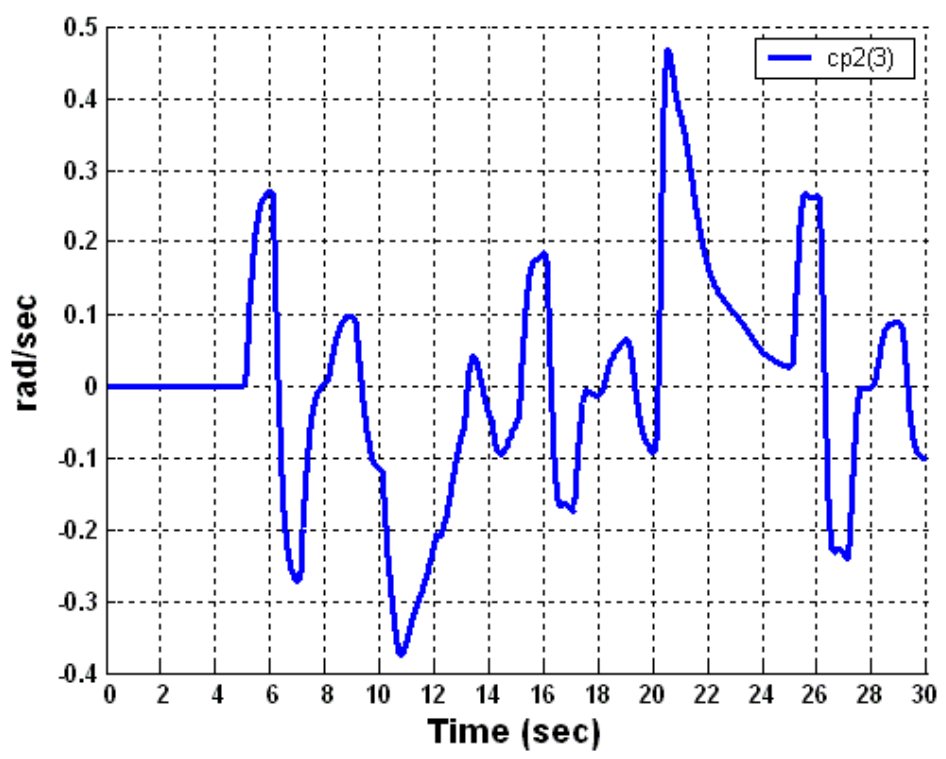

Figure 6.16 CP2 term: Pitch body signal

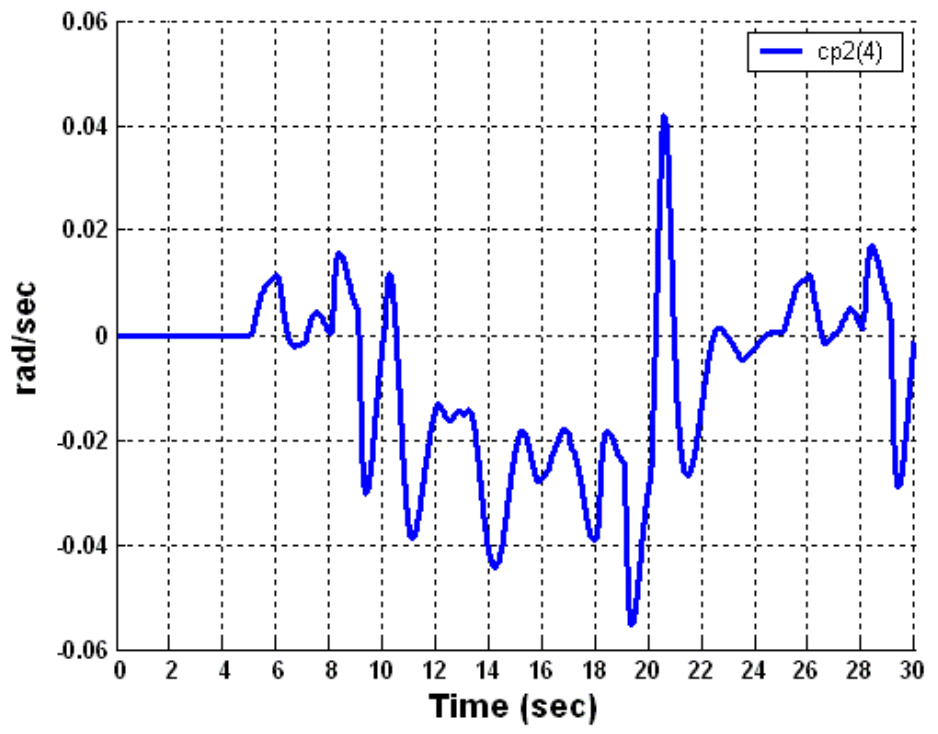

Figure 6.17 CP2 term: Yaw body signal 


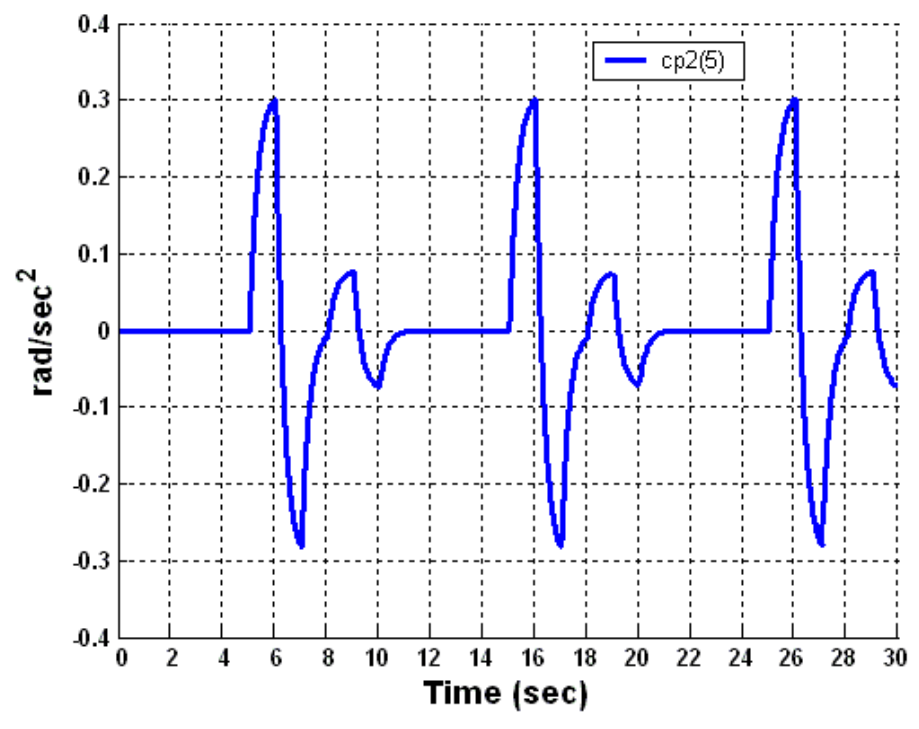

Figure 6.18 CP2 term: Reference model roll acceleration

In (6.12), $\alpha$ represent the angle of attack $(\mathrm{rad})$, and $\beta$ represents the sideslip $(\mathrm{rad})$. The following plots are generated using the 30 seconds of flight data collected from the phase II implementation of the IFCS flight simulator. Figure 6.19 shows the CP3 bias term that is set to a constant value of 1.05 . Figure 6.20 shows a plot of $\alpha$, the angle of attack. Figure 6.21 shows a plot of $\beta$, the sideslip. Using the CP1, CP2, and CP3 terms generated previously from 30 seconds of flight data, the product term,

Kronecker (or tensor) product is performed over the neural network input terms: CP1, $\mathrm{CP} 2$, and $\mathrm{CP} 3$. The product is referred as the BP terms. the $60(4 \times 5 \times 3) \mathrm{BP}$ terms are generated and plotted in Figure 6.22.

$$
\mathrm{BP}=((\mathrm{CP} 1 \otimes \mathrm{CP} 2) \otimes \mathrm{CP} 3)
$$


Another important term is the roll acceleration error term, Uperr. Figure 6.23 shows a plot of the Uperr term based on the 30 seconds of flight data collected from the phase II implementation of the IFCS flight simulator.

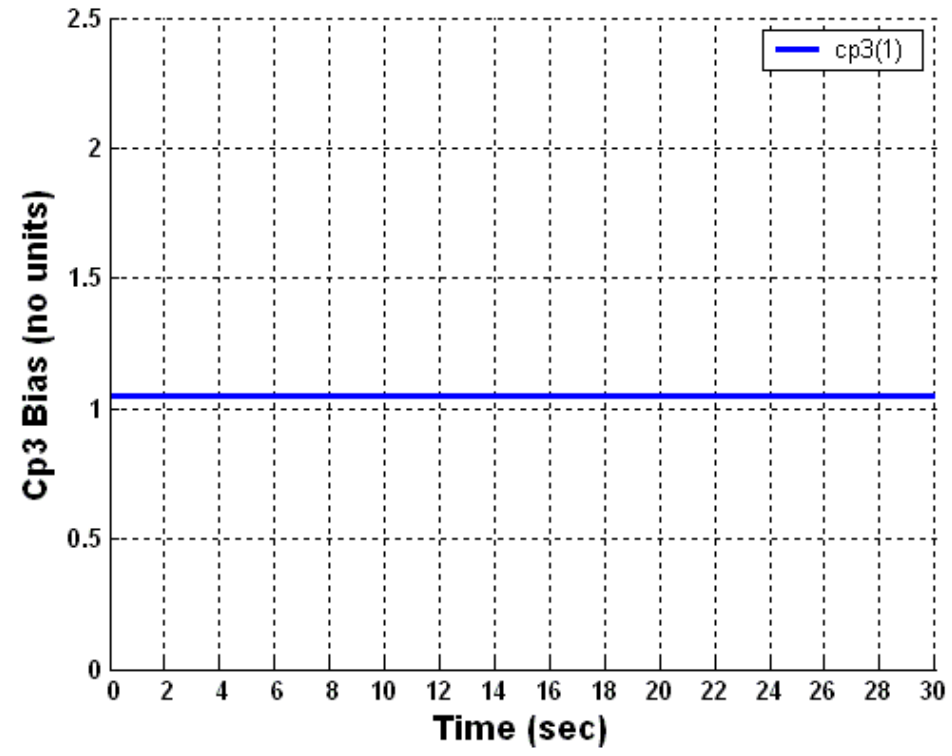

Figure $6.19 \mathrm{CP} 3$ bias term 


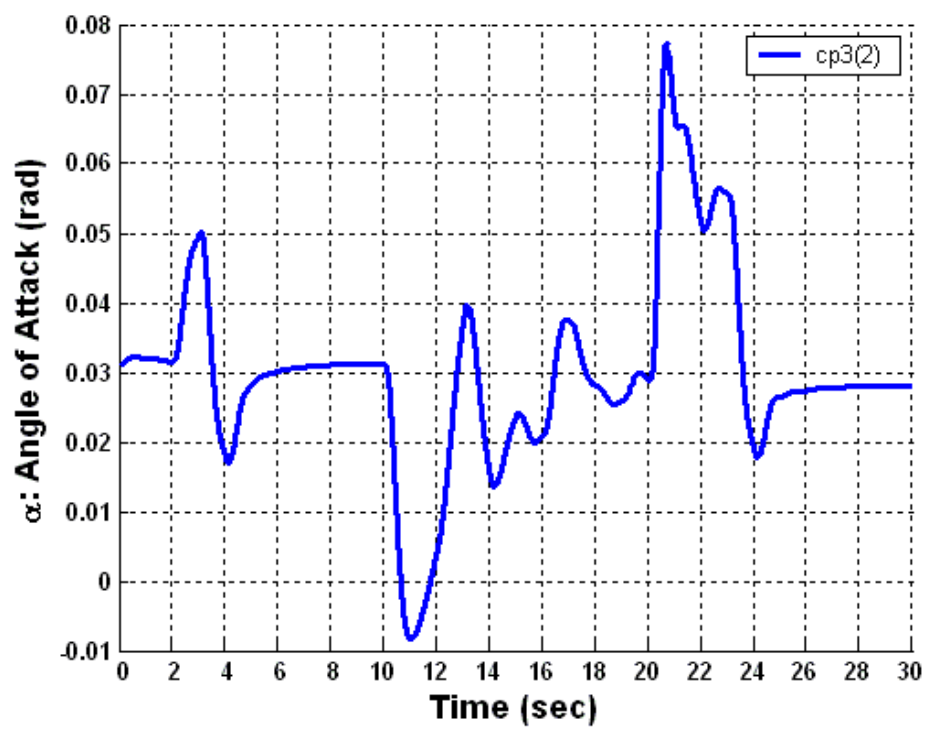

Figure $6.20 \mathrm{CP} 3$ term: angle of attack

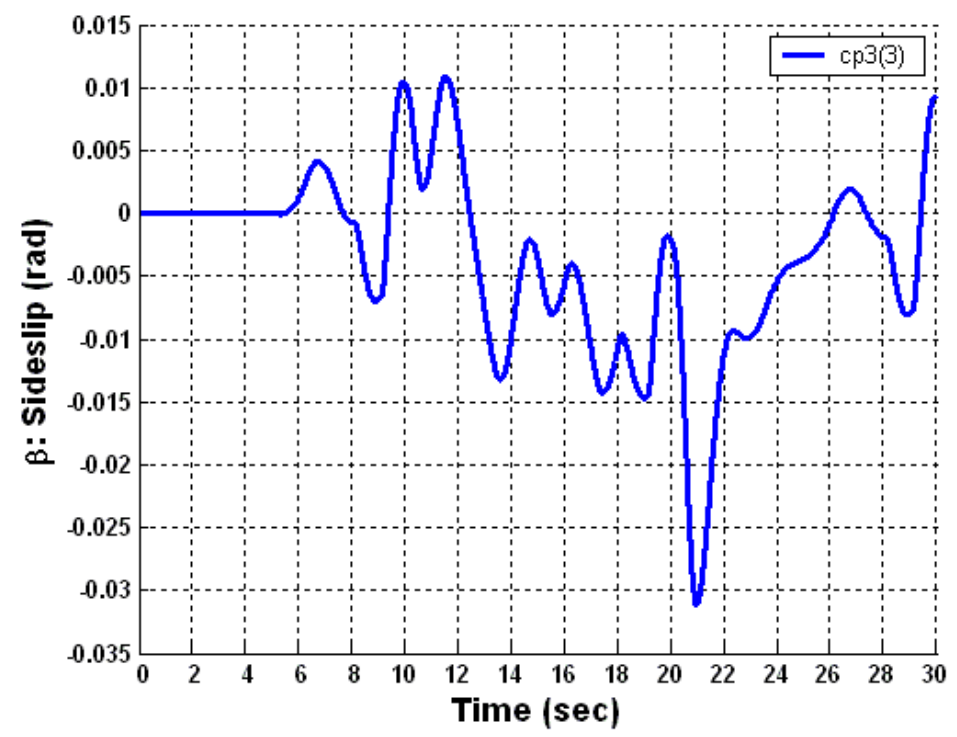

Figure 6.21 CP3 term: sideslip 


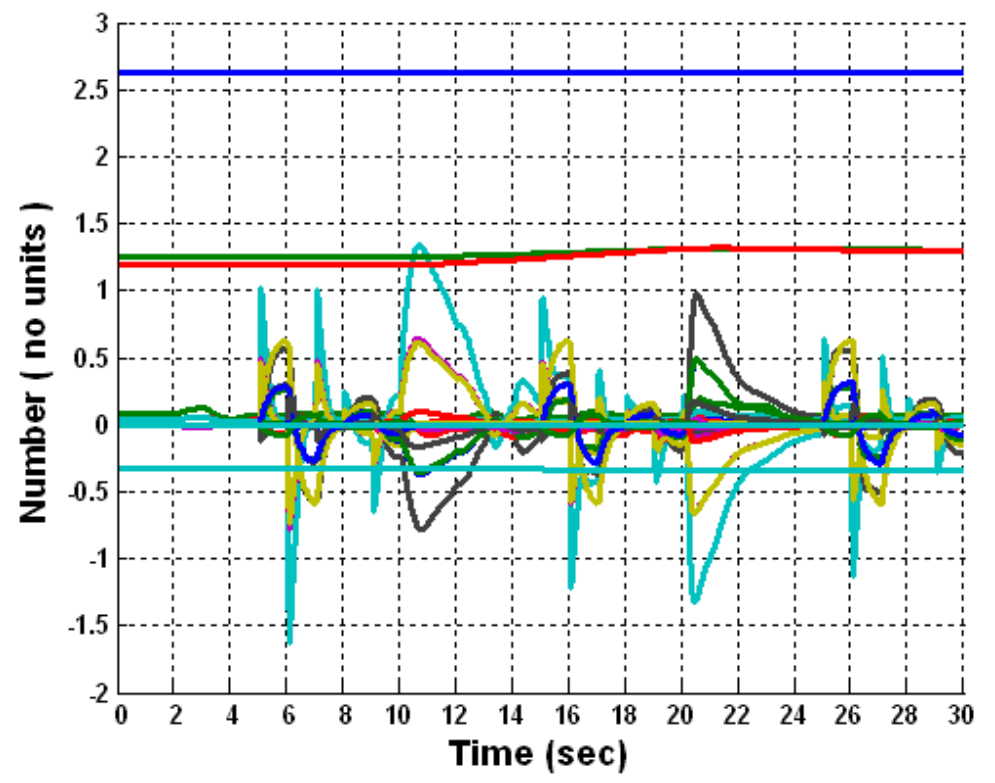

Figure 6.22 $60 \mathrm{BP}$ terms for the roll-channel S-Pi

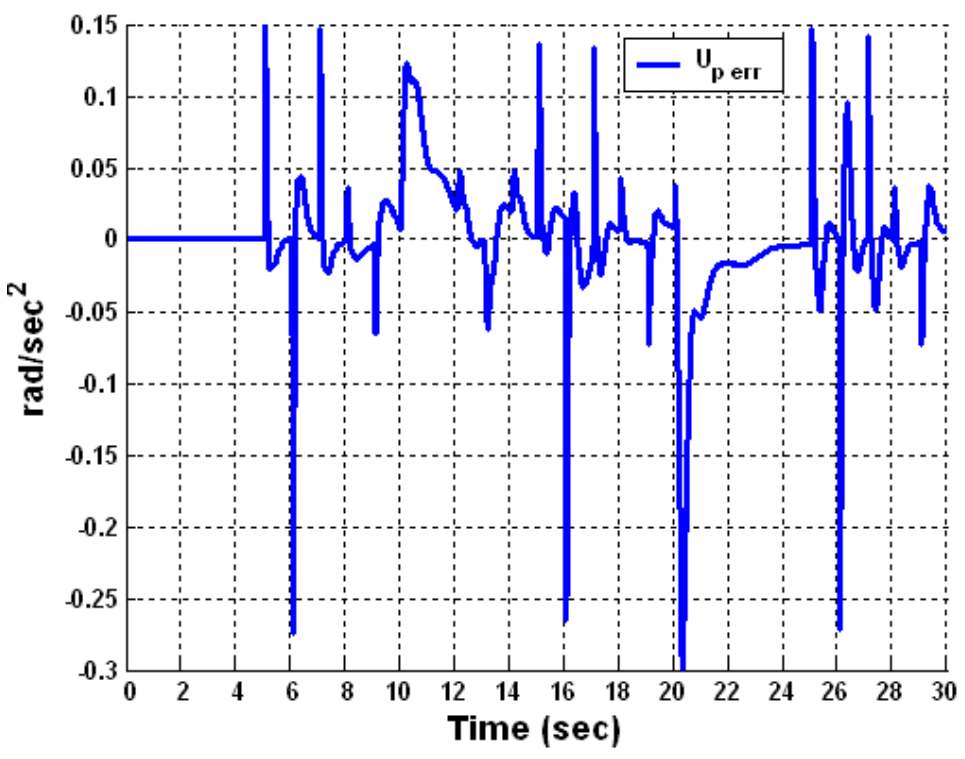

Figure 6.23 60 Acceleration error terms for the roll-channel S-Pi 


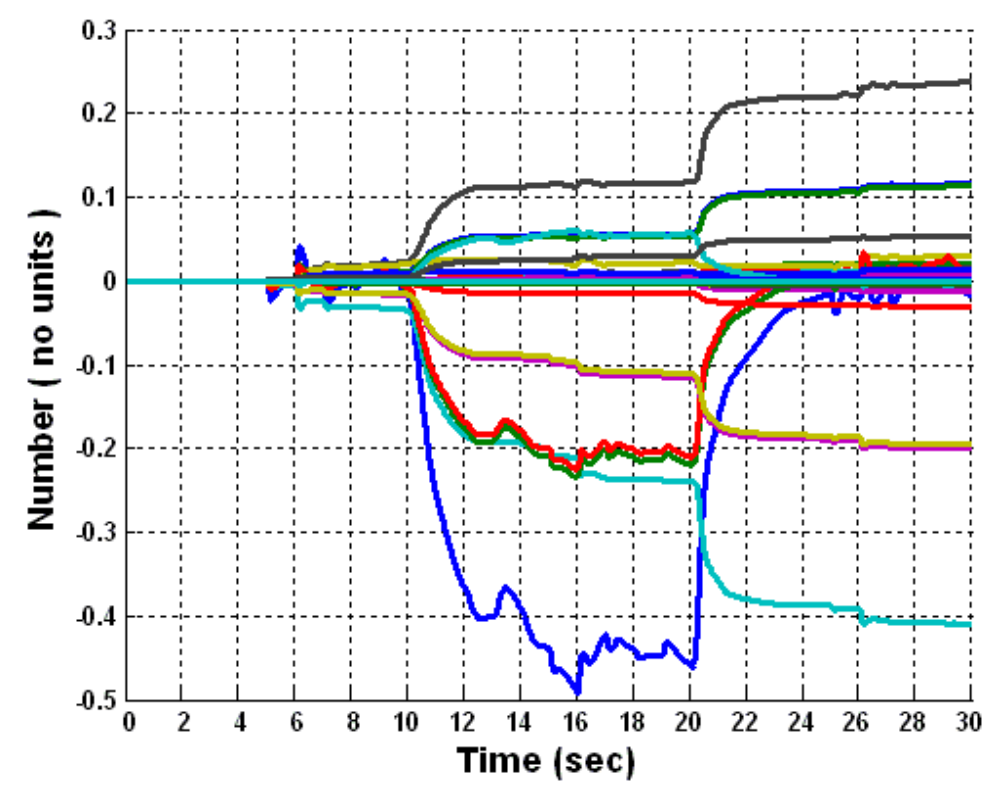

Figure 6.24 Adaptation of the 60 roll-channel S-Pi weight terms

The terms, Uperr and BP, are collectively used for the S-Pi neural network adaptation process. The adaptation is performed using the E-modified neural network learning algorithm (see Figure 6.6). Note that the adaptation term, $U e$, that is shown in Figure 6.6 is here the roll acceleration error term, Uperr.

A S-Pi neural network learning algorithm is implemented using the E-modified neural network learning algorithm (Figure 6.6). To adapt to the $60 \mathrm{BP}$ terms, the roll channel S-Pi neural network consists of 60 weight terms. The adaptation of the neural network weight terms over time is shown in Figure 6.24.

Since the roll channel S-Pi neural network, in this case, is a second order neural network, a stability monitoring system consisting of 3 monitors is implemented. The formulation of the monitors is based on the previous discussion (6.9). 


$$
\begin{aligned}
& \text { Monitor } 1=\operatorname{sum}\left\{\Delta W_{01}, \Delta W_{02}, \mathrm{~K}\right\} \\
& \text { Monitor } 2=\operatorname{sum}\left\{\Delta W_{11}, \Delta W_{12}, \mathrm{~K}\right\} \\
& \text { Monitor } 3=\operatorname{sum}\left\{\Delta W_{21}, \Delta W_{22}, \mathrm{~K}\right\}
\end{aligned}
$$

In (6.14), Monitors 1, 2, and 3 monitor the neural network adaptation changes that are caused by the zero order, first order, and the third order weight terms respectively. Using the 30 seconds of flight data collected from the phase II implementation of the IFCS flight simulator, the implemented S-Pi neural network learning algorithm is adapted. The terms, BP (Figure 6.22) and (Figure 6.23) serve as the S-Pi neural network input. Figure 6.25 shows online monitor 1 that indicates the weight changes due to the adaptation of the zero-order weight terms. Figure 6.26 shows online monitor 2 that indicates the weight changes due to the adaptation of the first order weight terms. Figure 6.27 shows online monitor 3 that indicates the weight changes due to the adaptation of the second order weight terms. A noteworthy point here is the trends of the monitors indicate no predominant spikes (greater than $10 \sigma$ ). This is because the neural network is responding to relatively smooth input terms.

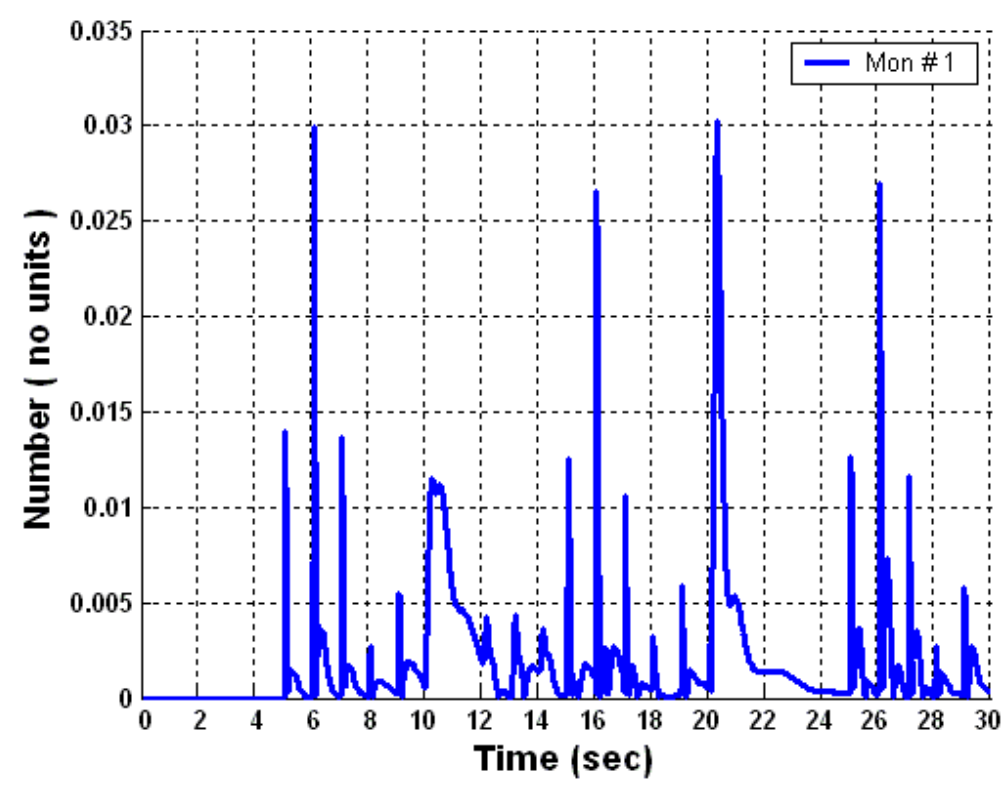

Figure 6.25 Online monitor 1: zero order weights adaptation monitor 


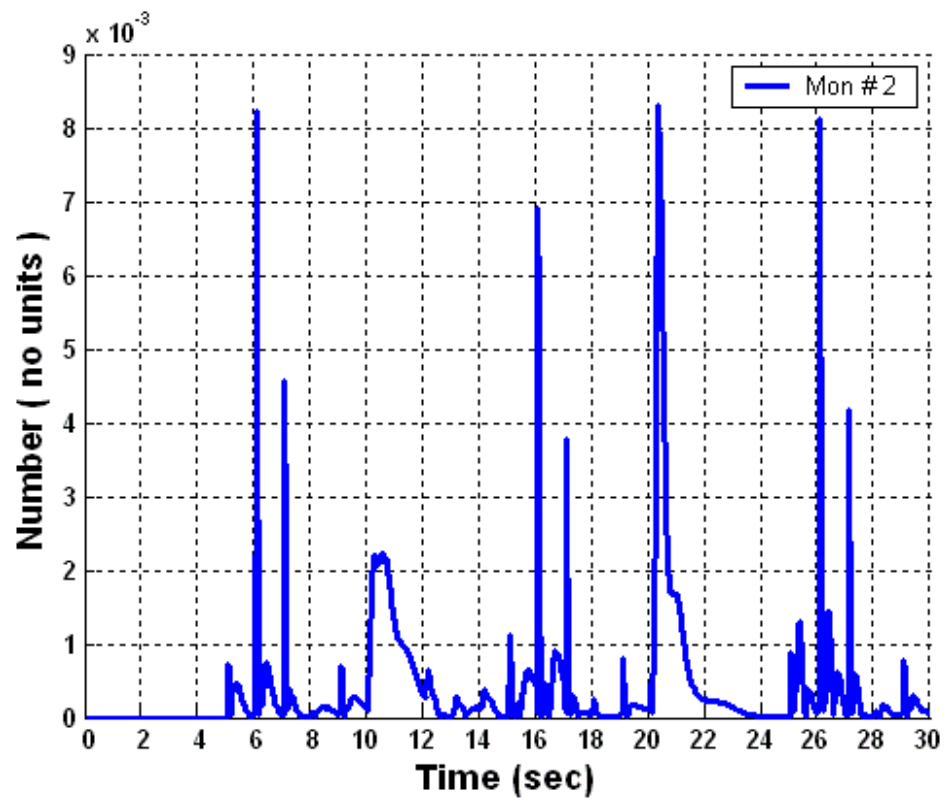

Figure 6.26 Online monitor 2: first order weights adaptation monitor

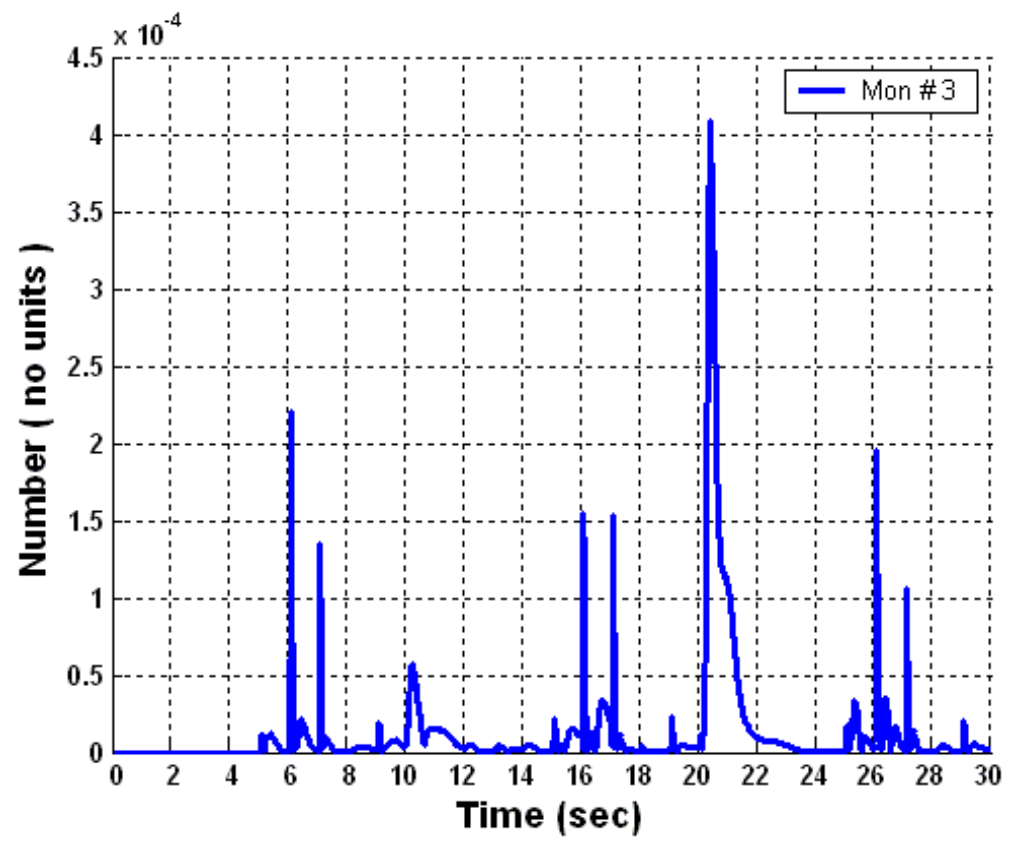

Figure 6.27 Online monitor 3: second order weights adaptation monitor 
In order to verify the correctness (in terms of implementation) of the S-Pi neural network learning algorithm, the output from the S-Pi neural network algorithm implemented here is compared to the output from the IFCS implementation of the S-Pi neural network. Figure 6.28 shows the roll-channel S-Pi neural network output, Upad obtained from the current implementation. Figure 6.29 shows the roll-channel S-Pi neural network output, Upad obtained from the IFCS implementation. It is evident from Figure 6.28 and Figure 6.29 that the output from the S-Pi neural network that is implemented here is identical to the output obtained from the IFCS implementation of S-Pi (from the flight simulator).

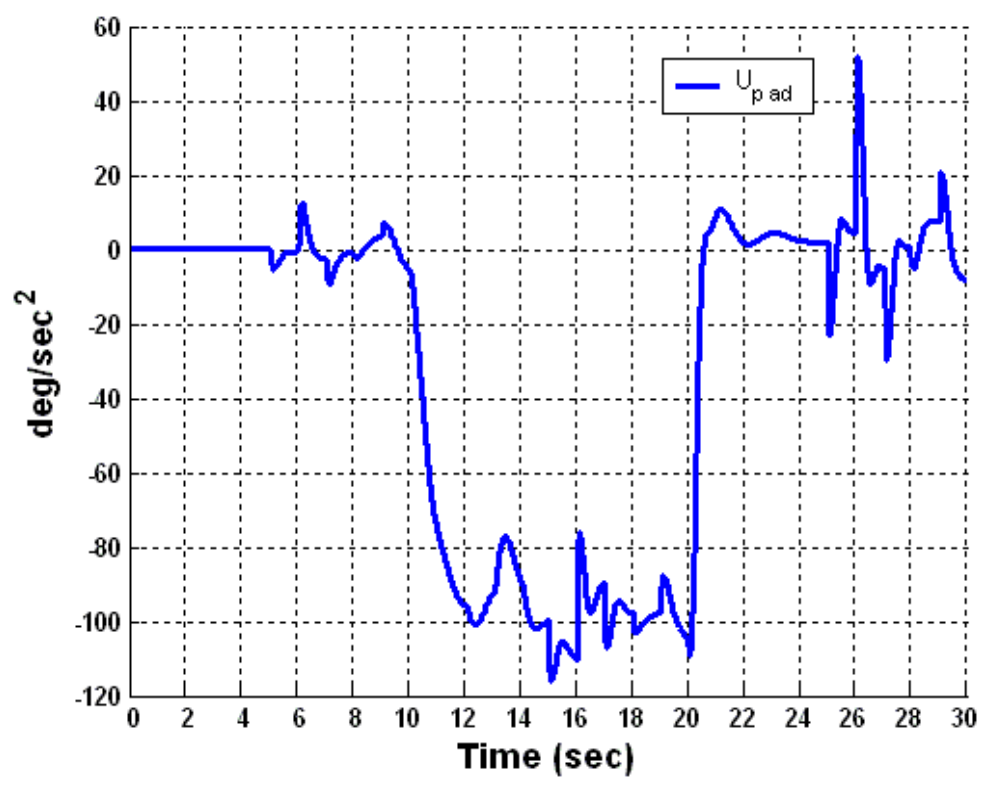

Figure 6.28 Output from the current implementation of roll channel S-Pi NN 


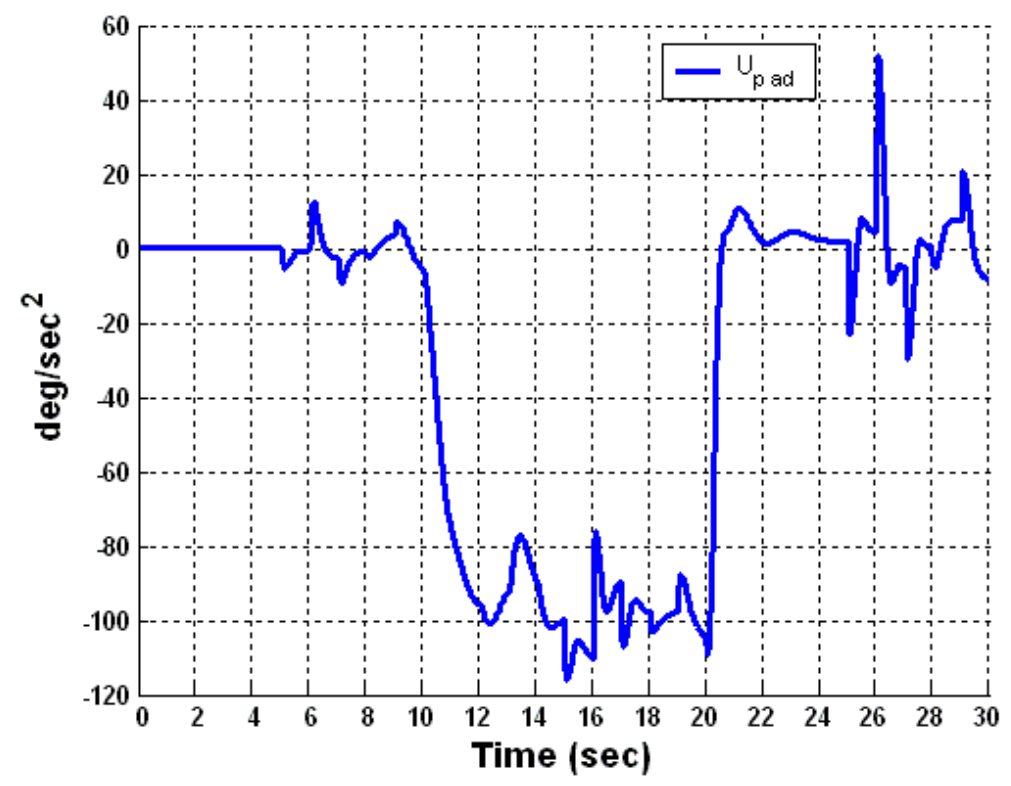

Figure 6.29 Output from the IFCS implementation of roll channel S-Pi NN

\subsection{Lyapunov Stability Analysis of ADALINE NN}

ADALINE (an acronym for ADAptive LInear NEuron) is an example of a feed forward neural network, which is named so as all the data flows in one direction, from the input nodes to the output nodes. ADALINE network was developed by Bernard Widrow and Marcian Hoff as an adaptive pattern classification machine to illustrate principles of adaptive behavior and learning $[114,115]$. The structure of ADALINE networks is same as perceptron networks, but the training in ADALINE is similar to that of backpropagation neural networks. In ADALINE, the learning procedure is based on an iterative search process, where error feedback is used to guide the search process. The diagram of a single ADALINE network with two inputs $\left(p_{1}, p_{2}\right)$ is shown in Figure 6.30. 


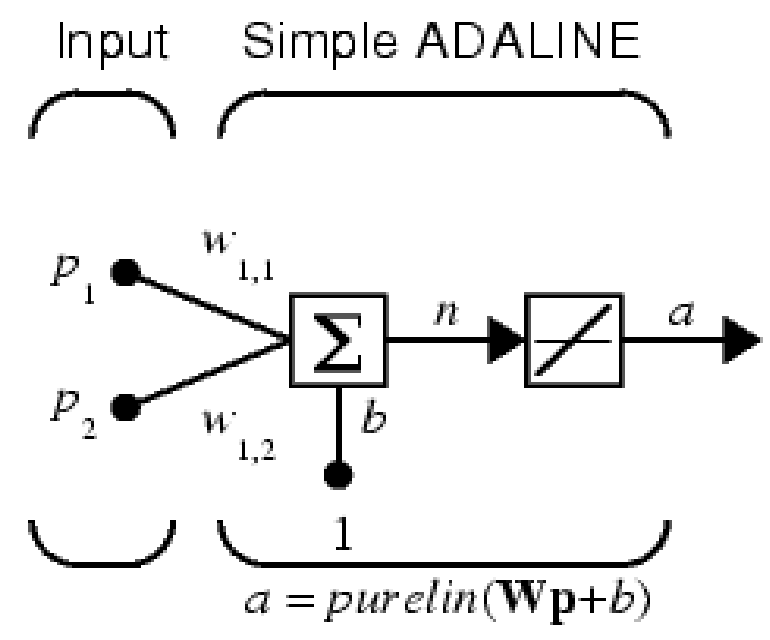

Figure 6.30 A single ADALINE neural network with two inputs

The Adaline is a modification of the Perceptron as it substitutes bipolar $(-1 /+1)$ for binary (0/1) inputs, and adds a bias weight. But the most important modification in ADALINE is the use of the delta rule of learning. As with the Perceptron, the delta rule in ADALINE compares the desired output to the actual output to compute the weight adjustments. However, the delta rule in ADALINE squares the errors and averages them to avoid negative errors cancel-out the positive ones. ADALINEs are based on the use of an attractive (as opposed to repulsive) goal seeking learning procedure in which a convergent sub-network output value is defined as the goal for each pattern. Consequently, the sub-network is expected to learn the proper weight values to produce that goal value for any given set of input patterns. This is in contrast to the later Perceptrons, which were repulsive driven meaning that a specified goal value is not defined for each sub-network only the misclassification error information was utilized. Less complete error-information can lead to reinforcement learning that only provides the information that an error or success has occurred without the magnitude or direction (type) of the error. Positive reinforcement is the attractive form while negative reinforcement is the repulsive form $[114,115]$. 
In 1962, Widrow found a learning procedure for ADALINE and called it the WidrowHoff learning rule, more commonly known as the Delta learning rule [114]. The learning rule is based on the realization that the greatest sources of the error are the active lines. Consequently, the Widrow-Hoff learning rule changes the value of each weight in proportion to its pre-weight line value (in this case 1 or 0 ) according to the following rule.

$$
\text { Weight change }=(\text { Pre-weight line value }) \frac{\text { Error }}{\text { Number of inputs }}
$$

The application of this rule results in an error when the same pattern is presented again, if any pre-weight line value is 0 (although repeated presentations will gradually reduce this error). If the error is conserved so that all of it is distributed to the weights then the error can be eliminated. Consequently, error conservation increases the rate of learning.

Instead of just using the pre-weight line value to indicate the greatest source of error a variation would be to include the weight values as well since the post-weight line value give a more accurate measure of error assignment. The trade-off is a slightly more complex learning rule and slower learning if the error value is not conserved since smaller numbers are the result. In fact, this variation is the rule used in the Back Propagation networks, commonly known as the Backpropagation learning rule.

With the Widrow-Hoff procedure, all input lines representing features that are common to the patterns will tend to zero $[114,115]$. This leaves the non-overlapping pattern features to define the convergent sub-network output value. Consequently, only one ADALINE is needed for any set of patterns making it a compact solution for a pattern set having patterns with some unique feature. Yet, not all pattern sets exhibit this property. For instance, consider the set having patterns: 111,011 , and 110 (the "exclusive or" problem). No single pattern has a unique feature meaning the ADALINE will never converge to zero error. In such situations, a modification of the Widrow-Hoff procedure called the Relaxation procedure is used. In relaxation procedure, the input values that are used to modify the weights are normalized (so that the weights add up to some constant number, usually one). This ensures that large 
patterns do not overly bias the learning. Not realized at the time was the significance of the error value used in the ADALINEs. Logical negation of error values produces multivalued and fuzzy logic certainty values, which allows downstream circuits to work with a degree of matching signal.

ADALINE and S-Pi are the online learning neural networks used in phase II of the IFCS implementation (refer to Figure 6.1). The adaptation rule followed in the IFCS implementation of the ADALINE neural network is the same as that in the S-Pi neural network, the E-modified neural network adaptation rule (refer to Figure 6.6). The IFCS implementation of the ADALINE neural network consists of a single linear neuron element. Similar to the IFCS implementation of the S-Pi neural network, the adaptation terms for IFCS implementation of the ADALINE consists of BP terms and the roll acceleration error term, Uperr. However, instead of $60 \mathrm{BP}$ terms, the IFCS ADALINE neural network implementation consists of $6 \mathrm{BP}$ terms and 6 Uperr terms.

22 seconds of flight data is collected from the ADALINE implemented IFCS flight simulator. Using the collected flight data, the 6 BP terms and the 6 Uperr terms are collected and shown here in Figure 6.31 and Figure 6.32 respectively. Observe that in Figure 6.32 the 6 Uperr terms that rise abruptly at 12.5 seconds. Figure 6.33 shows a plot of the abrupt increase in the 6 Uperr terms from 12 to 14 seconds. Since the ADALIE neural network is embedded within the simulator (see Figure 6.1), the neural network will likely respond to the changes in the Uperr terms.

The goal of the constructed stability monitoring system is to detect the deviations in the neural network response to variations in the input data. An ADALINE neural network learning algorithm is implemented that adapts to the input data using the E-modified neural network learning algorithm (Figure 6.6). The $6 \mathrm{BP}$ terms (Figure 6.31) and the 6 Uperr terms (Figure 6.32) serve as the neural network input and adaptation terms respectively. To adapt to 
the $6 \mathrm{BP}$ terms, the roll channel ADALINE neural network consists of 6 weight terms. The adaptation over time of the 6 neural network weight terms is shown in Figure 6.34.

As the roll channel ADALINE neural network, in this case, consists of 6 terms, a stability monitoring system consisting of 6 monitors is implemented. The formulation of the monitors is similar to that followed in the S-Pi neural network case (6.9). Figure 6.35, Figure 6.36, Figure 6.37, Figure 6.38, Figure 6.39, and Figure 6.40 show online monitor 1, 2, 3, 4, 5, and 6 respectively. In this case of learning, monitors 1 (Figure 6.35), 2 (Figure 6.36), 3 (Figure 6.37), 4 (Figure 6.38), and 6 (Figure 6.40) show predominant spikes in their values (greater than $10 \sigma$ ) around 12.5 seconds. Monitor 6 (Figure 6.39), however, did not show any predominant spikes in its values. This implies that the monitors $1-4$, and 6 were able to detect changes in the neural network response to variations in the input, but monitor 6 was unable to detect the change in the neural network adaptation.

In order to verify the correctness (in terms of implementation) of the ADALINE neural network learning algorithm, the output from the ADALINE neural network algorithm that is implemented here is compared to the output from the IFCS implementation of the ADALINE neural network. Figure 6.41 shows the roll-channel S-Pi neural network output obtained from the current implementation. Figure 6.42 shows the roll-channel S-Pi neural network output obtained from the IFCS implementation. It is evident from Figure 6.41 and Figure 6.42 that the output from the ADALINE neural network that is implemented here and the output obtained from the IFCS implementation of the ADALINE (from the flight simulator) is identical. 


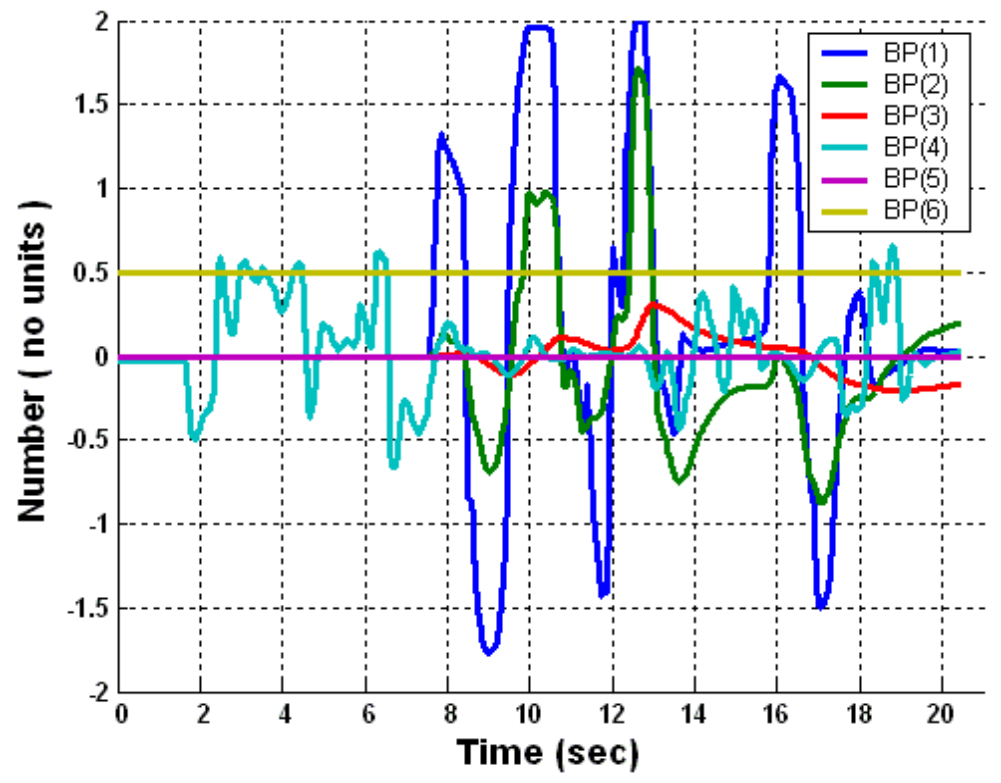

Figure 6.31 6 BP terms for the roll-channel ADALINE

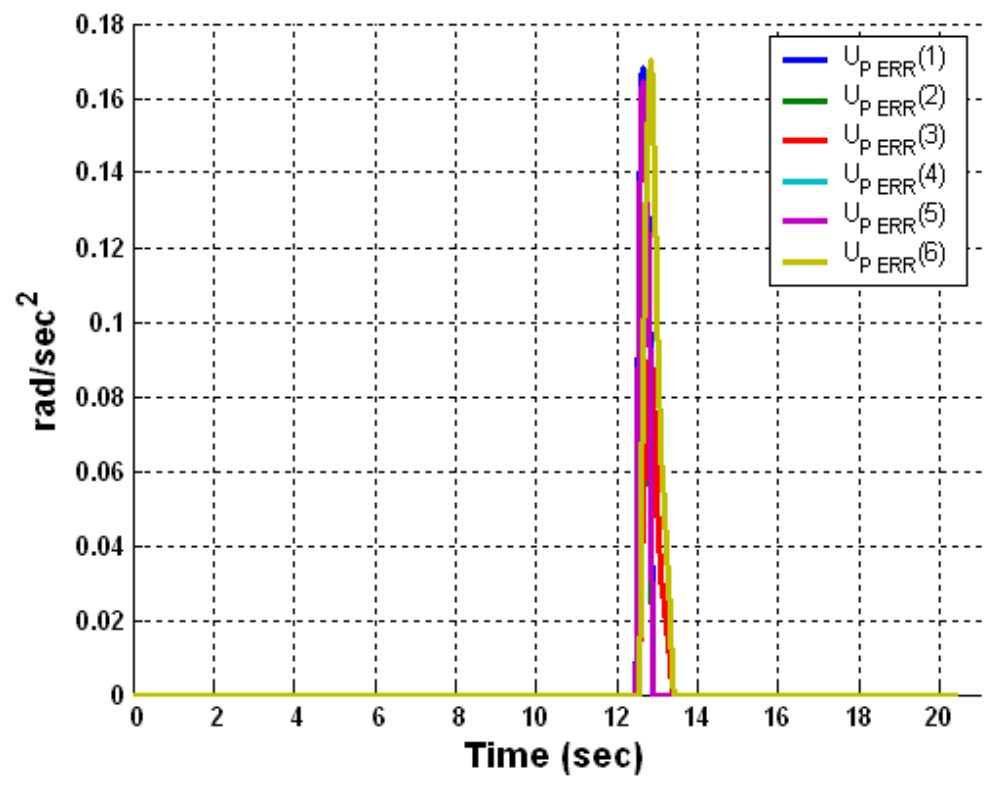

Figure 6.32 6 Acceleration error terms for the roll-channel ADALINE 


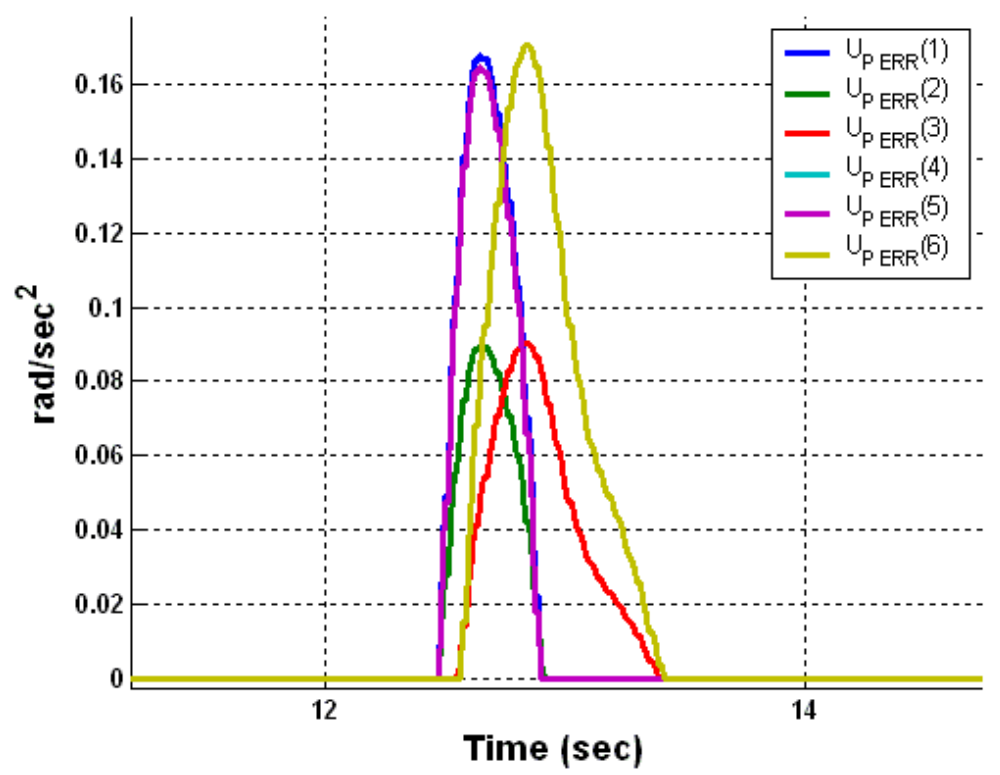

Figure 6.336 Roll channel acceleration error terms for times 12 to 14 seconds

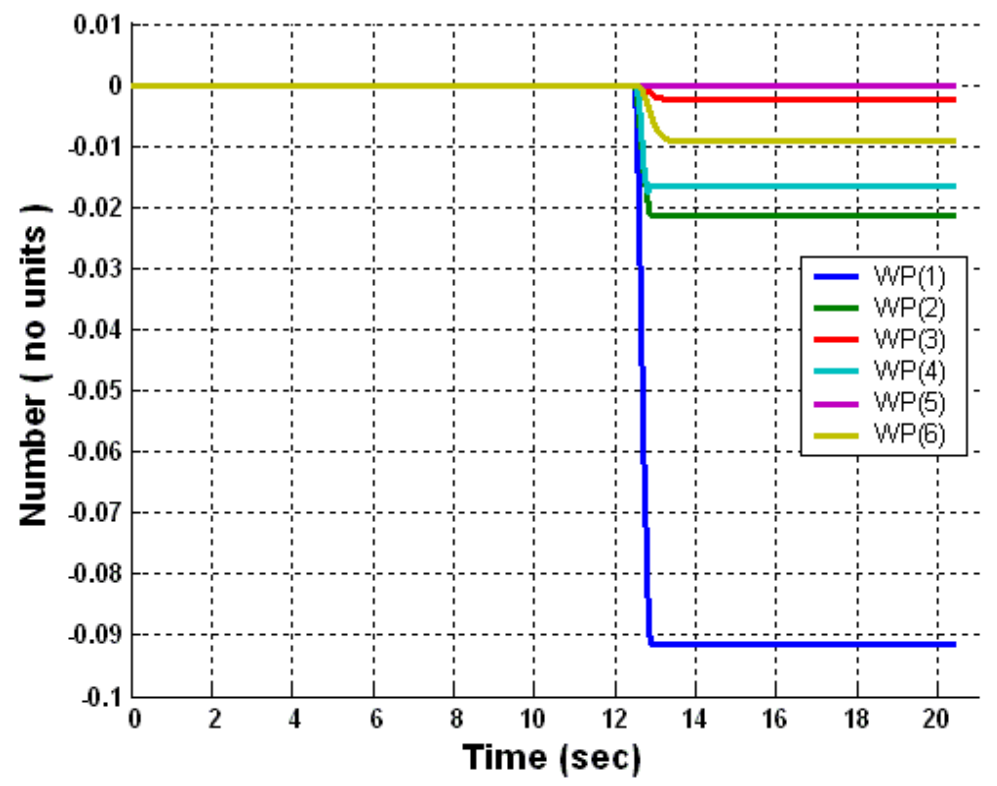

Figure 6.34 Adaptation of the 6 roll-channel ADALINE weight terms 


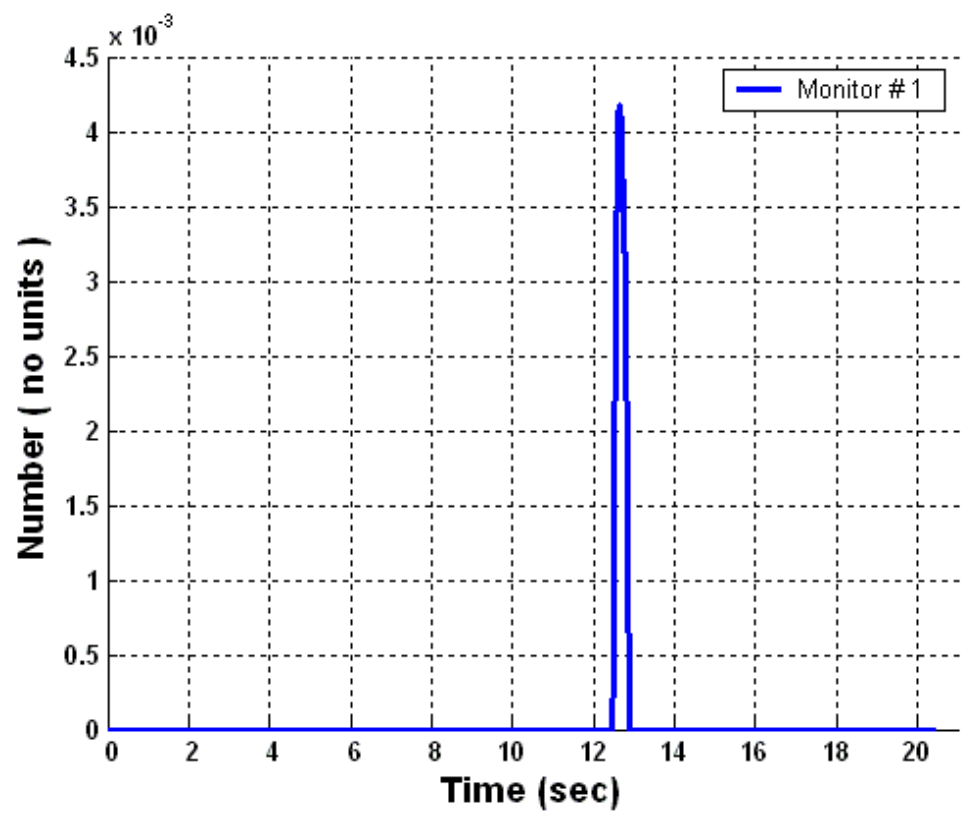

Figure 6.35 ADALINE online monitor 1

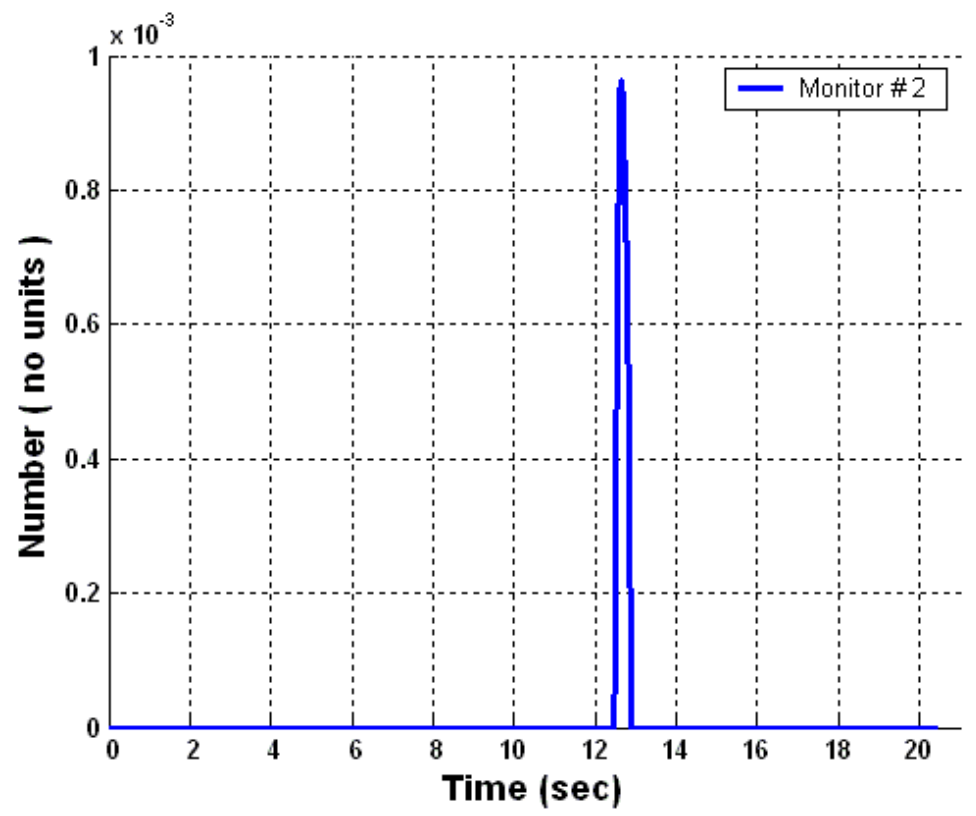

Figure 6.36 ADALINE online monitor 2 


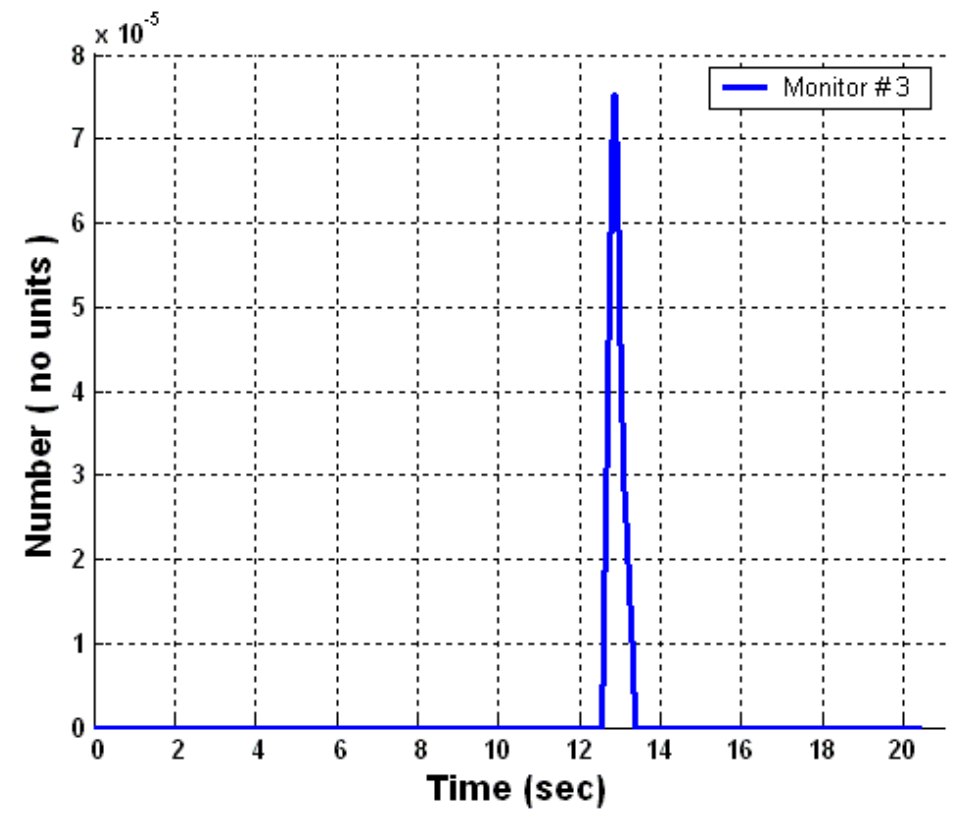

Figure 6.37 ADALINE online monitor 3

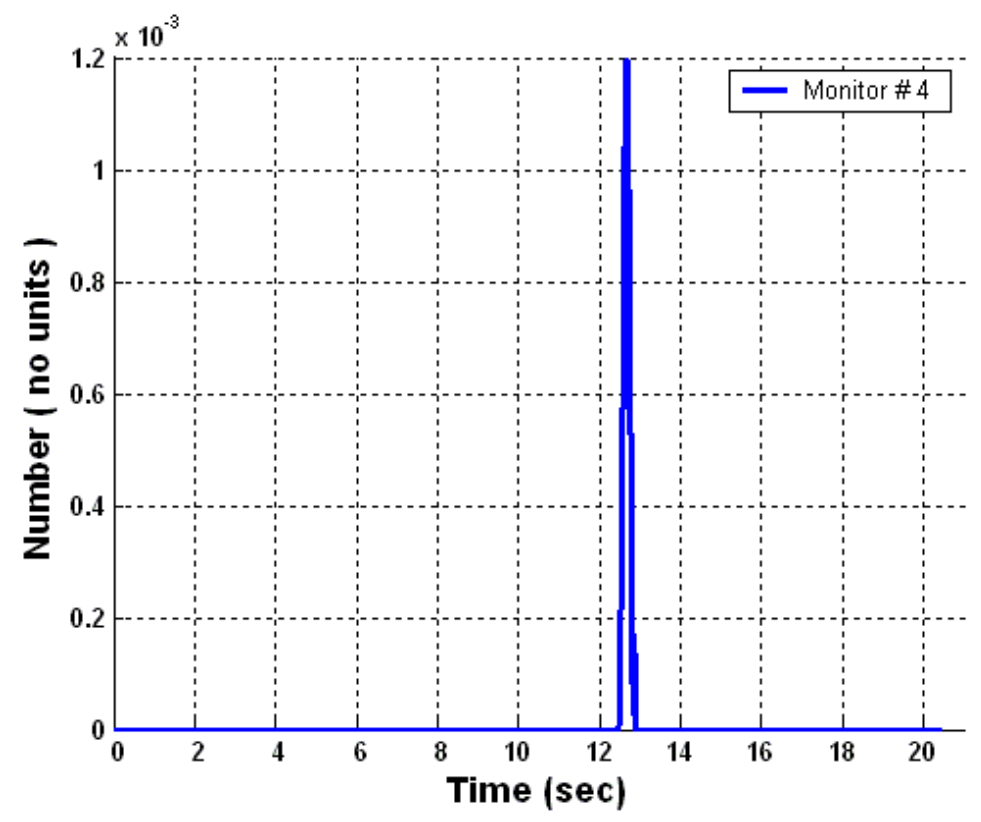

Figure 6.38 ADALINE online monitor 4 


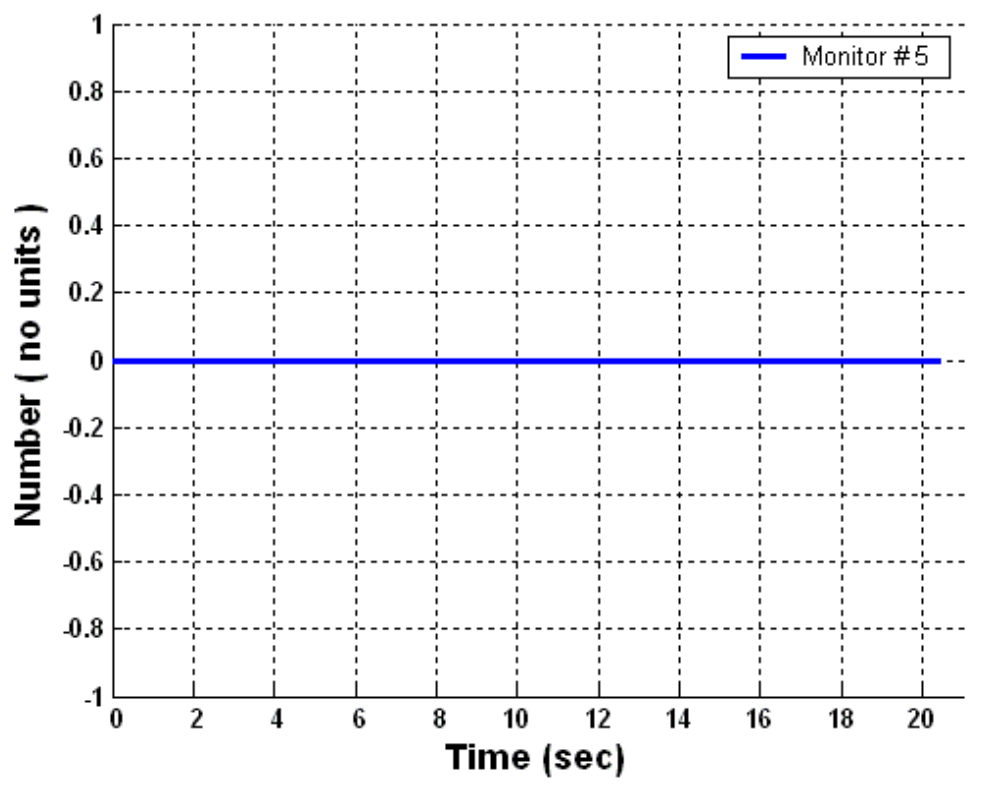

Figure 6.39 ADALINE online monitor 5

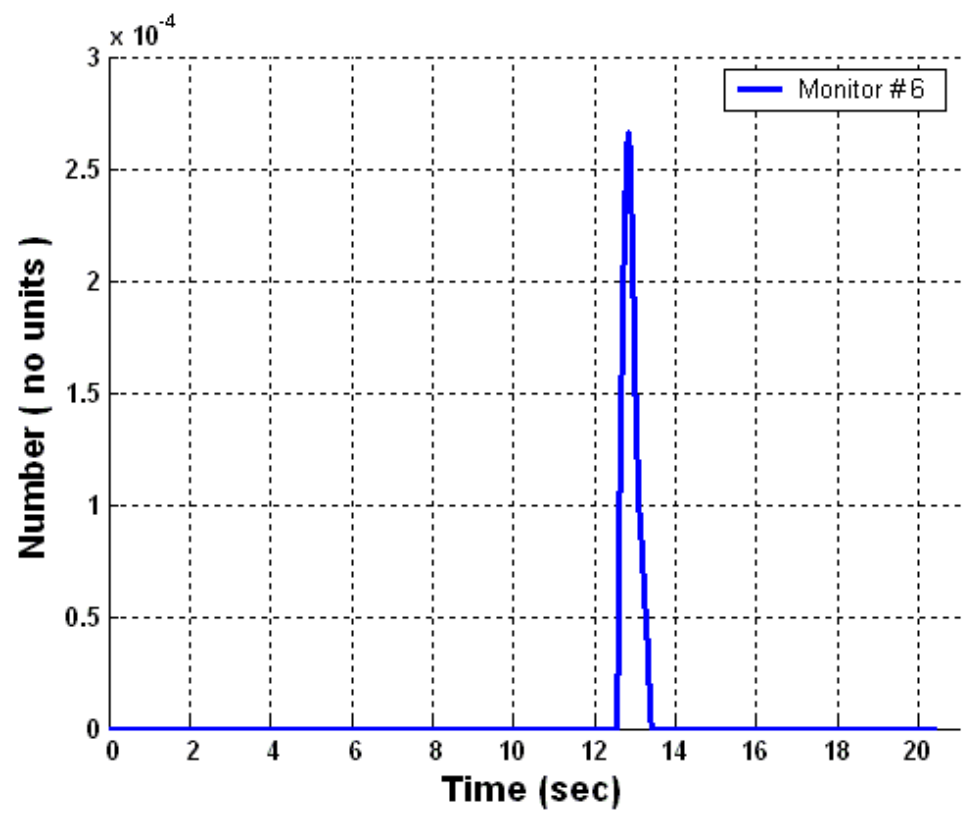

Figure 6.40 ADALINE online monitor 6 


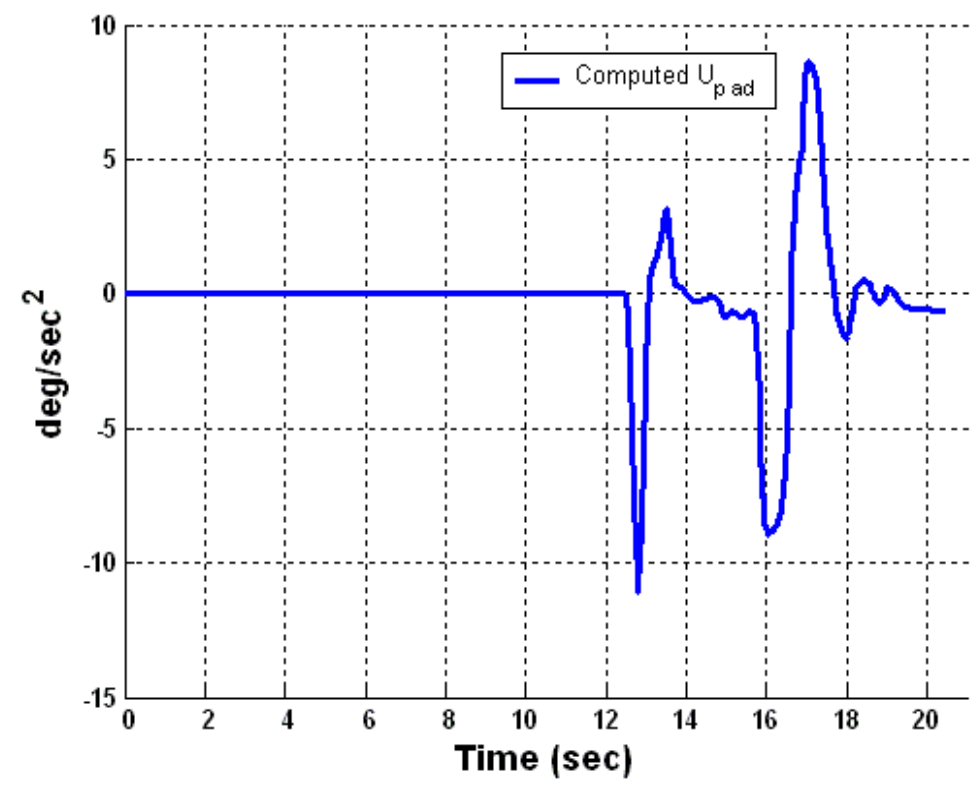

Figure 6.41 Output from the current implementation of roll ADALINE NN

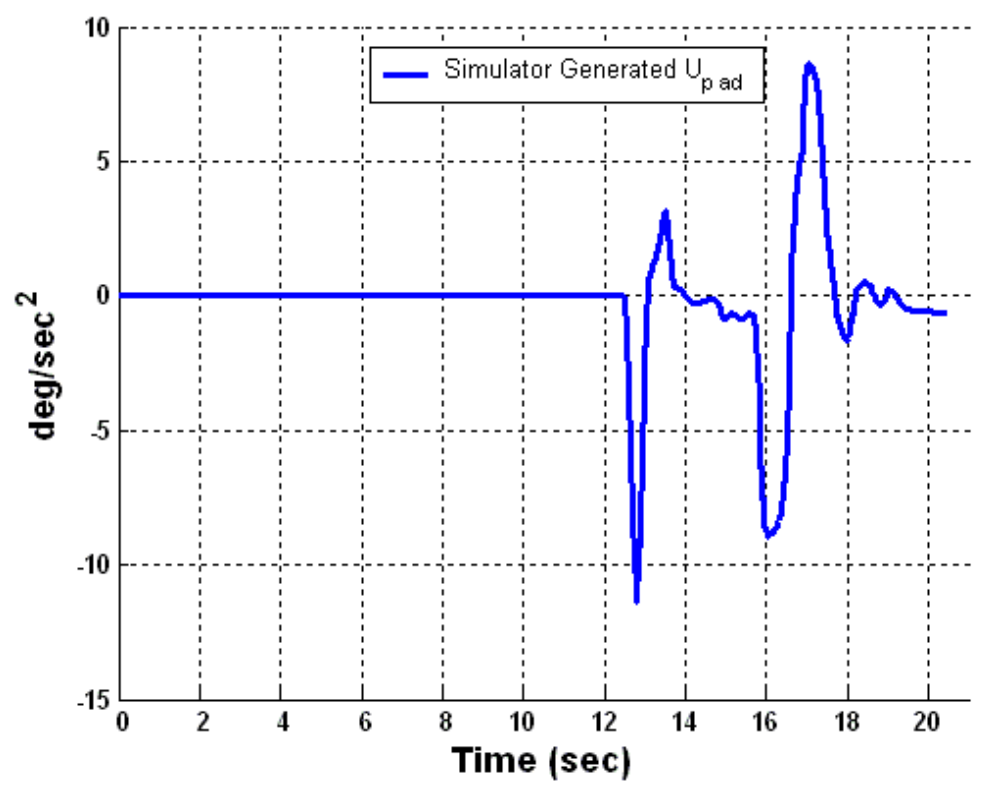

Figure 6.42 Output from the IFCS implementation of roll ADALINE NN 
Based on the proposition of considering stability as a heuristic measure of reliability in neural network adaptation, a non-conventional analysis approach for NN based online adaptation is developed in the form of online stability monitoring. In chapter 3 , some stability results for neural networks were provided in the form of mathematical stability proofs. These results are based on the premise of considering $\mathrm{NN}$ adaptation as a dynamical system behavior, and are obtained using the formulations of stability according to Lyapunov's theory. It must be realized that due to the system specific nature of Lyapunov stability analysis, the stability results provided in chapter 3 and the stability monitoring approach presented in chapter 4 are confined to the specific NN architecture, the DCS.

In this chapter, the stability analysis approach is generalized to S-Pi and ADALINE neural networks that represent feedforward and linear network architectures respectively. The results and the analysis presented, in this chapter, show that it is possible to apply and extend the Lyapunov stability analysis technique to a general class of neural networks. 


\section{Chapter}

\section{Conclusions and Future Work}

It is well understood that the performance of adaptive systems is significantly enhanced by the use of adaptive learning components such as online learning neural network algorithms. By accommodating for changing dynamics of the system, online adaptation plays a critical role in aiding the adaptive system to recuperate from catastrophic failure conditions. For instance, in a flight control system, these failures can be slow and catastrophic (sensor/actuator failures, changed aircraft dynamics: broken aileron or stabilator, etc) [40, 42, $43,48,49,50,83,85,86,88,91]$. When the neural networks perform online adaptation, its behavior may have a direct consequence on the overlaid adaptive system. Therefore, it is necessary to ensure a correct and safe neural network behavior before its deployment into the safety-critical system. The research identifies the growing needs for providing a safe and reliable platform for online adaptation in safety-critical applications $[1,5,6,10,14,69,73,80$, 82]. Embarking on the principle of analysis of online learning neural networks for safety critical systems, the research proposed and derived a detailed neural network analysis procedure for understanding the behavior of the neural networks to on-line adaptation.

Unlike other conventional learning paradigms, the learning process in most neural networks is dependant on the nature of the inputs and evolves non-deterministically over time in an unpredictable manner. In many cases of neural network learning, the inputs to the neural network and outputs from the neural network can be determined, but it is not clear how (and when) the neural network has achieved a certain desired state in learning. Due to this nondeterministic nature of adaptation, neural networks are considered "black box" learning mechanisms $[1,5,6,10,14]$. To overcome the problems encountered by traditional neural network analysis methods such as formal methods of testing, input/output monitoring, rule- 
extraction, etc the focus of the research is towards the development of a framework for a non-conventional procedure of testing and analysis of online learning neural networks.

It is observed here that the learning process in neural networks resembles in many ways, the behavior of dynamical systems $[15,16]$. As a result, the research is based on characterizing the learning behavior in adaptive neural networks in the context of dynamical systems. The idea behind characterizing adaptation in neural networks in the context of dynamical systems is that there are well-established dynamical system analysis techniques that can then be applied for analysis of neural networks. One of the foremost of the system analysis techniques is the Lyapunov stability theory. The interesting feature about Lyapunov's stability analysis is that it can be systematically applied to validate the existence (or nonexistence) of stable-states in a dynamical system [56, 65, 98, 107]. This research is based on considering stability of neural network adaptation as a heuristic measure of its correctness. Lyapunov stability analysis techniques that have been commonly used in understanding the stability properties of many dynamic systems are applied here for the analysis of online learning neural networks.

While there exist some results on the stability analysis of feedforward neural networks, it is a known problem that there is no generalized approach to derive stability results for more complex class of neural networks such as the Kohonen self-organizing maps, the Radial Basis Functions (RBF), and the Dynamic Cell Structures (DCS). The research overcame the challenge by providing an in-depth theoretical analysis of the stability properties of online adaptive DCS neural networks using the formulation of the Lyapunov stability theory. The motivation for the work is the phase-I of the IFCS project, where the DCS serves as the online learning neural network. The derived theoretical stability analysis approach is based on Lyapunov's second method. The technique is demonstrated in guaranteeing the correct adaptation and convergence of the DCS within a reasonable amount of time when exposed to specific representatives of data. The analytical and experimental results presented in Chapter 
3 demonstrate that a provably stable neural network guarantees the learning to converge to a stable state within a reasonable amount of time without bifurcating towards instability.

To understand the adaptation behavior of the neural network to unforeseen data representations, the research proposes an online stability monitoring system. The availability of online stability monitoring system, in such cases, for determining if (and when) the online learning neural network adaptation converges back to a stable state is expected to significantly enhance safety and reliability of the adaptive system. An online stability monitoring system consisting of four stability monitors is developed in Chapter 4. Each monitor is designed to monitor the adaptation behavior of the neural network due to various aspects of neural network leaning as discussed in Chapter 4. Experimental results presented in the research (Table 4-1) indicate that the developed stability monitoring system is capable of detecting atypical neural network adaptation and interpret a belief in the stability of online adaptation in a real-time manner.

Receiver operating characteristic (ROC) curves (otherwise known as 'relative operating characteristic' curves) that were developed in the 1950's as a by-product of research into making sense of radio signals contaminated by noise $[108,109]$. For the neural network stability monitoring and analysis, receiver operating characteristic (ROC) curves (otherwise known as 'relative operating characteristic' curves) are used to show the performance of the monitoring system as a trade off between selectivity and sensitivity of the failure detection (stability monitoring) mechanism. If the value of the fused monitors is above the threshold value $(10 \sigma)$, then the neural network response is considered atypical. From the ROC curves results presented in the research (Section 4.2 in Chapter 4), it can be observed that the developed stability monitoring system has the capability to detect unstable online learning behavior under changing flight conditions. By making a stability monitoring system available for online use in adaptive systems, one can significantly enhance the ability to analyze and understand the behavior of the embedded adaptive software component. It is important to 
realize that online stability monitoring is a technique that complements theoretical stability analysis (Chapter 3).

Performance indicators and convergence-time estimates are few of the many criteria that may be used as measures of neural network reliability. Performance indicators provide a measure of the ability of the neural network to perform a required function for a certain time. Convergence time estimates provide a measure of the rate at which the neural network learning returns to pre-specified error levels. It is, in general, a challenge to evaluate the performance and convergence in a neural network as the learning approach is stochastic and evolves in an unpredictable manner over time. The research has developed a methodology for generating indicators of performance for adaptation in the DCS. The indicators are based on the learning dynamics of the neural network as described by the stability monitors. The established methodology provides time estimates for the neural network convergence. The time estimates are generated based on current data conditions and the confidence in the neural network. The stability monitors provide the confidence in neural network adaptation. Analytical and experimental results are presented using examples of DCS learning from twinspiral data to indicate the accuracy of the developed convergence prediction method.

Topology preservation is an important property of information processing useful in many practical applications including exploratory data analysis, control systems, signal processing, image processing, pattern recognition, speech analysis, and computational topology. Topology preserving neural networks like the DCS are characterized by the ability to extract information encoded in the input data of arbitrary high-dimension into features using prototypes, called weight vectors. The feature of dynamic allocation of network size and shape allow growing self-organizing maps such as the DCS to extract information encoded in the input data more accurately, and more commonly with a fewer number of nodes than traditional Kohonen self-organizing maps with a predefined network size and shape [32]. 
The research identifies the selection of an appropriate neural network growing strategy for dynamic allocation of network size and shape as crucial for achieving topology preservation in G-SOMs [32, 37]. The research identifies some fundamental flaws in the implementation of most neural network growing algorithms that are outlined as follows. Most neural network growing strategies for G-SOMs are based on addition of nodes into the network between the node with the highest accumulated local error and its connected node (neighbor) $[30,35]$. While this process of dynamic allocation of network size and shape is, in general, shown to generate effective topology preserving mappings from a variety of input data distributions $[30,35]$. it was shown here that certain conditions of the data and/or the network can prompt growing strategies to add nodes into regions that represent no data. The heuristic results presented in Chapter 5 indicate that the introduction of nodes into regions representing no data can not only disrupt the topology preservation properties of the neural network, but also increase the computational effort on the neural network training algorithm to recover back to a topology preserving mapping. The research identifies the cause of the problem as neural network growing strategies not taking into account the distribution of the input data during the addition of nodes. Analytical and experimental results presented in Chapter 5 detail the conditions of the data and/or the network that lead to a disruption in topology preserving properties of the neural network.

The research observed that some existing neural network growing strategies for dynamic allocation of network size and shape suffer from the following flaw in the implementation of the algorithm. Under certain conditions of the neural network, the neuron with highest accumulated error is observed as being disconnected from the network. In other words, a neighbor for the node with the highest accumulated error may not always exist. Analytical and experimental results provided in Chapter 5 show that such conditions can result in implementation errors when neural network growing strategies add nodes in between the node with the highest accumulated error and its connected neighbor. 
To overcome the observed problems with existing dynamic allocation algorithms, the research has developed a modified neural network growing algorithm for dynamic allocation of network size and shape for G-SOMs. The proposed modifications allow the modified dynamic allocation algorithm to add nodes strictly into regions of the network containing data and more commonly into regions where the necessity for additional neurons is most required. The modifications also ensure that the newly introduced neuron forms a part of the existing neural network map. These modifications allow the modified neural network growing algorithm to overcome the common problems that exist with the process of dynamic allocation of network size and shape. The initial motivation for the development of the modified dynamic allocation algorithm was to overcome the problems existing with current neural network growing strategies. Analytical and experimental results presented in Chapter 5 indicate that not only does the modified growing algorithm overcomes these problems, but also generates efficient topology preserving mappings throughout the neural network learning process.

It is important to realize that due to the system specific nature of Lyapunov stability analysis, the Lyapunov functions and, therefore, the analytical stability results provided in Chapters 3 and 4 of the research are confined to the specific NN architecture, the DCS. The research established a methodology to generalize the developed stability analysis techniques to a general class of neural network architectures, namely feedforward, higher order and linear neural networks. The motivation for the work is the phase-II of the IFCS, where the Sigma-Pi (S-Pi) and ADALINE neural networks serve (and replace the DCS) as the online learning neural networks.

The S-Pi neural networks represent higher order networks, where each layer contains a higher-order term. Often the layers have summation units fed via weighted connections by intermediate product unit outcomes. The research provided an extension of the Lyapunov stability analysis for the S-Pi neural networks (Chapter 1). Lyapunov function candidates for the S-Pi neural network have been derived. 
An $N^{\text {th }}$ order S-Pi neural network may consist of $0,1^{\text {st }}, 2^{\text {nd }}, \mathrm{K}, N^{\text {th }}$ order networks, but the highest network order (or cardinally of the inputs) is $N$. The research has developed an online stability monitoring system consisting of $N+1$ monitors suitable for an $N^{\text {th }}$ order $\mathrm{S}$ $\mathrm{Pi}$ neural network. The mathematical formulation of the stability monitors is provided in Chapter 6. The IFCS implementation consists of third order S-Pi neural networks with each network containing up to 60 weight terms. A stability monitoring system consisting of 4 stability monitors, namely zero-order, first-order, second-order, and third-order monitors has been developed for the analysis of S-Pi neural networks. The mathematical formulations of the monitors are provided in Chapter 6. The zero-order monitor monitors the adaptation changes in the S-Pi neural network that are caused by the adaptation of zero order weight terms. Similarly, the first-order, second-order, and third-order monitors monitor the adaptation changes in the S-Pi neural network that are caused by the adaptation of first-order, secondorder, and third-order weight terms respectively. Based on the data collected from the IFCS flight simulator, a S-Pi neural network is implemented. Experimental results indicate that the developed monitors are capable of detecting changes in neural network responses to variations in the input data streams.

The ADALINE (ADAptive LINEar) neural networks are an example of linear feed forward networks, which are named, so as all the data flows in one direction, from the input nodes to the output nodes. The Adaline neural networks are essentially single-layer backpropagation networks. The research provides an extension of the Lyapunov stability analysis for the ADALINE neural networks (Chapter 1). Lyapunov function candidates for the ADALINE neural network have been derived.

The ADALINE neural network used in the IFCS consists of a single linear neuron. The neural network adaptation rule followed in the IFCS implementation of the ADALINE neural networks is the same as that followed in the S-Pi neural networks. However, instead of 60 weight terms, the IFCS ADALINE neural network implementation is split into 6 weight terms. A 
stability monitoring system consisting of 6 stability monitors for monitoring each individual weight term has been developed. The mathematical formulations of the stability monitors are provided in Chapter 1. Based on the data collected from the IFCS flight simulator, an ADALINE neural network is implemented. Experimental results indicate that the developed monitors are capable of detecting changes in neural network responses to variations in the input data streams.

The research identifies that the inapplicability of traditional analysis techniques for the evaluation and analysis of adaptive learning components such as the online learning neural networks for safety-critical applications as a serious safety concern. The focus of the research has been development of nonconventional analysis technique suitable for testing and analysis of online learning neural networks for safety-critical adaptive systems. The research presents a non-conventional analysis approach that is based on the concept of considering stability of online adaptation as a heuristic measure of correctness in the operation of the adaptive component for the sake of system safety. The scientific contribution of the research is the development of a framework for the analysis and evaluation of non-deterministic neural network adaptations in safety-critical adaptive systems using known analysis techniques such as the Lyapunov stability theory. The research recommends that adaptation mechanisms such as online learning neural networks undergo a rigorous testing and analysis phase before their deployment into the safety-critical systems.

\section{1. $\quad$ Future Work}

In the current work, the mathematical derivations of the stability of the DCS that are provided in Chapter 3 are applicable for the case of the DCS learning from stationary (or nonvarying) manifolds of data. In the future, the work on mathematical analysis of the stability of the DCS can be extended for the case of the DCS learning from non-stationary (or variable) manifolds of data. An online stability monitoring system is proposed and developed in the 
current work to provide an understanding of the stability of the DCS learning from variable manifolds of data. The four monitors that are proposed in Chapter 4 monitor the stability of the DCS by measuring the adaptation changes in the positions of the BMU, SBU, NBR, and NonNBR neurons. In the future, monitors that can monitor the connectivity structure encompassing the BMU, SBU, NBR and Non-NBR neurons can be developed to augment the online stability monitoring system. A methodology for neural network convergence prediction has been established in the current work to provide estimates of the neural network's returnto-stability after the introduction of data disturbances. The analysis presented in the current work is limited to single point data disturbances, i.e. for the introduction of a single data point outside the convex hull of the neural network training data. In the future, the convergence prediction analysis can be extended neural network's return-to-stability after the introduction of multiple point data disturbances. In the current work, the mathematical stability analysis techniques developed originally for understanding the stability properties of the DCS have been modified to generalize their application to the Sigma-Pi and the ADALINE neural networks. The Sigma-Pi represents feedfoward neural network architecture and the ADALINE represents linear neural network architecture. In the future, the generalized analysis techniques developed here can be applied to investigate the stability properties of other types of neural network architectures. A modified neural network growing process has been developed in the current work. The motivation for the proposed modifications is the flaws that are observed in the current implementations of neural network growing strategies. The current work presented a comparison study of the topology preserving properties of the modified and the original neural network growing process. In the future, the topology preserving properties of the modified neural network growing process can be compared to other emerging types of neural network growing processes. 


\section{Bibliography}

[1] J. Hull, D. Ward, and R. R. Zakrzewski. "Verification and validation of neural networks for safety-critical applications". In proceedings of the American Control Conference, Vol.6, No.8-10, pp. 4789-4794 May 2002.

[2] A. Krogh, and J. Vedelsby. "Neural network ensembles, cross validation, and active learning". In proceedings of Advances in Neural Information Processing Systems (NIPS'95), Vol. 7, pp. 231-238, 1995.

[3] M. Lowry, M. Boyd, and D. Kulkarni. "Towards a theory for integration of mathematical verification and empirical testing". In proceedings of the 13th IEEE International Conference on Automated Software Engineering, pp. 332-331, October 1998.

[4] G. E. Peterson. "A foundation for neural network verification and validation". SPIE Science of Artificial Neural Networks II, pp.196-207, 1966.

[5] J. Schumann, P. Gupta, and S. Nelson. "On verification \& validation of neural network based controllers". In proceedings of the Engineering Applications of Neural Networks (EANN'03) 2003.

[6] J. Schumann, and S. Nelson. "Towards V\&V of neural network based controllers". Workshop on Self-Healing Systems, 2002.

[7] D. Marjorie, B. J. Taylor, and S. Skias. "Rule extraction from dynamic cell structure neural network used in a safety critical application." In proceedings of the 17th International FLAIRS Conference. Miami Beach, FL, 17-19 May 2004.

[8] B. J. Taylor, and M. A. Darrah. "Verification and validation of neural networks: a sampling of research in progress." In proceedings of the AeroSense, 2003.

[9] B. Cukic, B. J. Taylor, and H. Singh. "Automated generation of test trajectories for embedded flight control systems." International Journal of Software Engineering and Knowledge Engineering, Vol.12, No. 2, pp. 175-200, 2002.

[10] Orna Raz. "Validation of online artificial neural networks - an informal classification of related approaches". A technical report for the NASA Ames Research Center, 2000.

[11] D. D. Gobbo, and B. Cukic. "Validating online neural networks". A technical report submitted to the Lane Dept. of Computer Science and Electrical Engineering, West Virginia University, December 2001.

[12] M.A. Boyd, J. Schumann, G. Brat, D. Giannakopoulou, B. Cukic, and A. Mili. "Validation and verification process guide for software and neural nets". A technical report for the NASA Ames Research Center, 2001.

[13] D. Mackall, S. Nelson, and J. Schumann. "Verification and validation of neural networks of aerospace applications". A technical report for the NASA Ames Research Center, NASA-CR-211409, 2002.

[14] "NASA guidebook for safety critical software". Technical Report, NASA-GB-1740.1396, 1996. 
[15] S. Yerramalla, E. Fuller, and B. Cukic, M. Mladenovski. "Lyapunov analysis of neural network stability in an adaptive flight control system". In proceedings of the Sixth Symposium of Self-Stabilization Systems (SSS'03), June 2003.

[16] S. Yerramalla, E. Fuller, and B. Cukic. "Lyapunov stability analysis of DCS quantization error". In proceedings of the IEEE International Joint Conference on Neural Networks (IJCNN'03), July 2003.

[17] J. Bruske, and G. Sommer. "Online learning with dynamic cell structures". In proceedings of the International Conference on Artificial Neural Networks (ICANN'95), Vol. 2, pp. 141-146, Paris, 1995.

[18] J. Bruske, and G. Sommer. "Dynamic cell structures learns a perfectly topology preserving map". In proceedings of the advances in Neural Information Processing Systems (NIPS'95), Vol. 7, No. 4, pp. 845-865, 1995.

[19] T. Kohonen. "Automatic formation of topological maps of patterns in a self-organizing system". In proceedings of the Conference on Image Analysis, pp. 214-220, 1981.

[20] T. Kohonen. "Self-organized formation of topologically correct feature maps". Biological Cybernetics, Vol. 43, No. 1, pp. $59-69,1982$.

[21] T. Kohonen. "Analysis of a simple self-organizing process". Biological Cybernetics, Vol. 44, No. 2, pp. 135-140, 1982.

[22] T. Kohonen, "Self-Organizing Maps". Springer, Berlin, Heidelberg, 1995.

[23] M. Cottrell, J.C. Fort, and G. Pages. "Theoretical aspects of the SOM algorithm". Neurocomputing Journal, Vol. 21, No. 1-3, pp.119-138, November 1998.

[24] J. Rahmel. "On the role of topology for neural network interpretation". In proceedings of the European Conference on Artificial Intelligence, 1996.

[25] K. Funahashi. "On the approximate realization of continuous mapping by neural network". Neural Networks Journal, Vol. 2, pp. 183-192, 1989.

[26] Yi Sun. "On quantization error of self-organizing map network". Neurocomputing Jorunal, Vol. 34, pp.169-193, 2000.

[27] T. Heskes. "Energy functions for self-organizing maps". In E. Oja and S. Kaski, editors, Kohonen Maps, pp. 303-316, Elsevier, Amsterdam, 1999.

[28] H. Ritter, and K. Schulten. "Kohonen's self-organizing maps: exploring their computational capabilities". In proceedings of the International Joint Conference on Neural Networks (IJCNN'88), pp. 109-116, San Diego, USA, 1988.

[29] M. J. D. Powell, "Approximation Theory and Methods". Cambridge, U.K., Cambridge Univ. Press, 1981.

[30] B. Fritzke. "A growing neural gas network learns topologies". In proceedings of the advances in Neural Information Processing Systems (NIPS'95), Vol. 7, pp. 625-632, 1995.

[31] B. Fritzke. "Growing Cell Structures - A self-organizing network for unsupervised and supervised learning". Neural Networks Journal, Vol. 7, No. 9, pp. 1441-1460, 1994. 
[32] B. Fritzke. "Growing self-organizing networks - why?" In proceedings of the European Symposium on Artificial Neural Network (ESANN'96), pp. 61-72, 1996.

[33] B. Fritzke. "A self-organizing network that can follow non-stationary distributions". In proceedings of the International Conference on Artificial Neural Networks (ICANN'97), Lausanne 1997.

[34] B. Fritzke. "Unsupervised clustering with growing cell structures". In proceedings of the IEEE International Joint Conference on Neural Networks (IJCNN'91), pp. 531-536, 1991.

[35] B. Fritzke. "Growing Grid - A self-organizing network with constant neighborhood range and adaptation strength". Neural Processing Letters, Vol. 2, No. 5, pp. 9-13, 1995.

[36] S. Marsland, J. Shapiro, and U. Nehmzow. "A self-organizing network that grows when required”. Neural Networks Journal, Vol. 15, pp. 1041-1058, 2002.

[37] T. Martinetz, and K. Schulten. "Topology representing networks". Neural Networks Journal, Vol. 7, No. 3, pp. 507-522, 1994.

[38] J. Park, and I. W. Sandberg. "Universal approximation using radial-basis function networks". Neural Computations Journal, Vol. 3, pp. 246-257, 1991.

[39] T. Heskes, and B. Kappen. "Online learning processes in artificial neural networks", Mathematical Foundations of Neural Networks, Elsevier, pp. 199-233, Amsterdam, 1993.

[40] C. C. Jorgensen. "Feedback linearized aircraft control using dynamic cell structures". World Automation Congress ISSCI-050.1, Anchorage Alaska, 1991.

[41] I. Kanellakopoulos, P.V. Kokotovic, and A.S. Morse. "Systematic design of adaptive controllers for feedback linearizable systems". IEEE Transactions on Automation and Control, Vol. 36, No. 8, pp. 1241-1253, 1991.

[42] M. Napolitano, C. D. Neppach, V. Casdorph, S. Naylor, M. Innocenti, and G Silvestri. "A neural network-based scheme for sensor failure detection, identification and accommodation". AIAA Journal of Control and Dynamics, Vol. 18, No. 6, pp. 12801286, 1995.

[43] M. Napolitano, G. Molinaro, M. Innocenti, and D. Martinelli. "A complete hardware package for a fault tolerant flight control system using on-line learning neural networks". IEEE Control Systems Technology, 1998.

[44] K. S. Narendra, and K. Parthasarathy. "Identification and control of dynamic systems using neural networks". IEEE Transactions on Neural Networks, Vol. 1, No.1, pp. 427, 1990.

[45] M.G. Perhinschi, G. Campa, M.R. Napolitano, M. Lando, L. Massotti, and M.L. Fravolini. "A simulation tool for on-line real time parameter identification". In proceedings of the AIAA Modeling and Simulation Conference, 2002.

[46] S. S. Sastry, and A. Isidori. "Adaptive control of linearizable systems". IEEE Transactions on Automatic Control, Vol. 34, No. 11, pp. 1123-1131, 1989. 
[47] D. G. Taylor, P. V. Kokotovic, R. Marino, and I. Kanellakopoulos. "Adaptive regulation of nonlinear systems with unmodeled dynamics". IEEE Transactions on Automation and Control, Vol. 34, No. 4, pp. 405-412, 1989.

[48] F. W. Burcham, Jr., T. A. Maine, C. G. Fullerton, and L. D. Webb. "Development and flight evaluation of an emergency digital flight control system using only engine thrust on an F-15 airplane". NASA TP-3627, Sept. 1996.

[49] The Boeing Company. "Intelligent Flight Control: advanced concept program". Project report, 1999.

[50] Institute for Scientific Research, Inc. "Dynamic cell structure neural network report for the intelligent flight control system". Technical Report, Document ID: IFC-DCSRD002-UNCLASS-010401, January 2001.

[51] H. P. Whitaker, J. Yamron, and A. Kezer. "Design of model reference adaptive control systems for aircraft". Instrumentation Laboratory, M.I.T. (1958).

[52] W. L. Brogan. "Modern Control Theory", II Edition, Prentice Hall Inc., 07632.

[53] S. Sastry, and M. Bodson. "Adaptive control: stability, convergence and robustness". Prentice-Hall Advanced Reference Series (Engineering), Englewood Cliffs, NJ, 1989. http://www.ece.utah.edu/ bodson/acscr.

[54] Bernard Friedland. "Advanced Control System", Prentice Hall Inc., 1996.

[55] Flavio Nardi. "Neural network based adaptive algorithm for nonlinear control". PhD thesis, School of Aerospace Engineering, Georgia Institute of Technology, 2000.

[56] M. W. Mc.Conley, B. D. Appleby, M. A. Dahleh, and E. Feron. "Computational complexity of Lyapunov stability analysis problems for a class of nonlinear systems". Industrial and Applied Mathematics Journal of Control and Optimization, Vol. 36, No. 6, pp. 2176-2193, 1998.

[57] K. M. Passino, N. Michel, and P. J. Antsaklis. "Lyapunov stability of a class of discrete event systems". IEEE Transactions on Automatic Control, Vol. 39, No. 2, February 1994.

[58] N. Rouche, P. Habets, and M. Laloy. "Stability theory by Lyapunov's direct method". Applied Mathematical Sciences, Vol. 22, Springer-Verlag, New York-Heidelberg, 1997.

[59] Oliver Theel. "An exercise in proving self-stabilization through Lyapunov functions". In proceedings of the 21st International Conference on Distributed Computing Systems (ICDCS'01), 2001.

[60] Wen Yu, and X. Li. "Some stability properties of dynamic neural networks". IEEE Transactions on Circuits and Systems: Part-1, Vol. 48, No. 2, pp. 256-259, 2001.

[61] Xinghuo Yu, M. Onder Efe, and Okyay Kaynak. "A Backpropagation Learning Framework For Feedforward Neural Networks". In proceedings of the IEEE International Symposium on Circuits and Systems (ISCAS'01), Vol. 3, pp. 700-702, Sydney Australia, May 2001. 
[62] V. I. Zubov. "Methods of A. M. Lyapunov and their applications". U.S. Atomic Energy Commission, 1957.

[63] Lecture notes on "Stability of dynamic systems", University of Pittsburgh, MAEM535, 2002.

[64] Lecture notes on "Dynamic Systems", Massachusetts Institute of Technology, Department of Electrical Engineering and Computer Science, 6.241, Recitation 6, 2002.

[65] P. R. Pagilla, Lecture notes on "Digital Control Systems", Oklahoma State University, Mechanical and Aerospace Engineering Department, MAE/ECEN 5473, 2002.

[66] CMU Archive of Machine Learning Benchmark Data Repository. http://www2.cs.cmu.edu/afs/cs/project/airepository/ai/areas/neural/bench/cmu/, 2002.

[67] S. E. Fahlman. "CMU Benchmark collection for neural net learning algorithms". Carnegie Mellon Univ., School of Computer Science, Machine-Readable Data Repository, Pittsburgh, 1993.

[68] M. Hagan, H. Demuth, and O. De Jesus, "An introduction to the use of neural networks in control systems". International Journal of Robust and Nonlinear Control, Vol. 12, No. 11, pp. 959-985, September, 2002.

[69] Institute for Scientific Research, Inc. (ISR). "Software verification and validation plan for the Airborne Research Test System II Intelligent Flight Control Program", IFCSVVP-F001-UNCLASS-120100, December 2000.

[70] Institute for Scientific Research, Inc. (ISR). "Toward reliable neural network software for the development of methodologies for independent verification and validation of neural networks", IVVNN-LITREV-F001-UNCLASS-111202, November 2002.

[71] Institute for Scientific Research, Inc. (ISR). "Introduction to development of methodologies for independent verification and validation of neural networks". IVVNNINT-F001-UNCLASS-021403, January 2003.

[72] Institute for Scientific Research, Inc. (ISR). "Draft guidance for the independent verification and validation of neural networks", IVVNN-GUIDE-D001-UNCLASS101603, October 2003.

[73] J. Smith. "Certification of on-line learning neural networks". In proceedings of the $7^{\text {th }}$ IASTED International Conference on Artificial Intelligence and Soft-Computing (ASC'03), July 2003.

[74] IEEE/EIA Guide - Industry Implementation of ISO/IEC 12207. "1995, Standard for Information Technology - Software Life Cycle Processes - Life cycle data". IEEE/EIA 12207.1-1997.

[75] IEEE/EIA Guide - Industry Implementation of ISO/IEC 12207. "1995, Standard for Information Technology - Software Life Cycle Processes - Implementation considerations". IEEE/EIA 12207.2-1997

[76] A. Mili, B. Cukic, Y. Liu, and R. Ayed. "Towards the verification and validation of online adaptive systems". In Computational Methods in Software Engineering, T. Khoshghoftaar, editor. Kluwer Scientific Publishing, 2003. 
[77] A. Avizienis. "The n-version approach to fault-tolerant software". IEEE Transactions on Software Engineering, Vol. 11, No. 12, pp. 1491-1501, 1985.

[78] V. Cortellessa. "Certifying adaptive flight control software". In proceedings of the $2^{\text {nd }}$ International Software Assurance Certification Conference (ISACC'00), 2000.

[79] G. Papadopoulos, P. Edwards, and A. Murray. "Confidence estimation methods for neural networks: a practical comparison". In proceedings of the European Symposium on Artificial Neural Networks (ESANN'00), pp. 75-80, 2000.

[80] L. H. Ungar, R. D. D. Veaux, and E. Rosengarten. "Estimating prediction intervals for artificial neural networks". In proceedings of the $9^{\text {th }}$ Yale Workshop on Adaptive and Learning Systems, 1996.

[81] W. Wen, J. Callahan, and M. Napolitano. "Towards developing verifiable neural network controllers". In proceedings of the Workshop on Al for Aeronautics and Space, 1996.

[82] R. R. Zakrzewski. "Verification of a trained neural network accuracy". In proceedings of the International Joint Conference on Neural Networks, pp. 1657-1662, 2001.

[83] R. Rysdyk and A. Calise. "Fault tolerant flight control via adaptive neural network augmentation". American Institute of Aeronautics and Astronautics AIAA-98-4483, pp. 1722-1728, 1998.

[84] S. Lee. "Neural network based adaptive and its applications to aerial vehicles". PhD thesis, Georgia Institute of Technology, School of Aerospace Engineering, Atlanta, GA, April 2001.

[85] C. C. Jorgensen. "Direct adaptive aircraft control using neural networks". Technical Report TM-47136, NASA Ames Research Center, CA, 1997.

[86] Manu Sharma. "A neuro-adaptive autopilot design for guided munitions". PhD thesis, Georgia Institute of Technology, School of Aerospace Engineering, Atlanta, GA, April 2001.

[87] Naira Hovakimyan, Flavio Nardi, and Anthony J. Calise. "A novel observer based adaptive output feedback approach for control of uncertain systems". IEEE Transactions on Automatic Control, Vol. 47, No. 8, pp. 1310-1314, 2002.

[88] Nakwan Kim. "Improved methods in neural network-based adaptive output feedback control, with applications to flight control". PhD thesis, Georgia Institute of Technology, School of Aerospace Engineering, Atlanta, GA, November 2003.

[89] Anthony Calise, Naira Hovakimyan, and Moshe Idan. "Adaptive output feedback control of nonlinear systems using neural networks". In Automatica Special issue Neural Networks for Feedback Control, Vol.37, No.8, 2001.

[90] Michael B. McFarland. "Adaptive nonlinear control of missiles using neural networks". PhD thesis, Georgia Institute of Technology, School of Aerospace Engineering, Atlanta, GA, July 1997. 
[91] Nakwan Kim, Anthony Calise, Naira Hovakimyan, J.V.R. Prasad, and Eric Corban. "Adaptive output feedback for high-bandwidth control of an unmanned helicopter". Journal of Guidance, Control and Dynamics, Vol. 25, No. 6, pp. 993-1002, 2002.

[92] Naira Hovakimyan, Flavio Nardi, Anthony Calise, and Nakwan Kim. "Adaptive output feedback control of uncertain systems using single hidden layer neural networks". IEEE Transactions on Neural Networks, Vol. 13, No. 6, pp. 1420-1431, 2002.

[93] R. Zbikowski and P. J. Gawthrop. "A survey of neural networks for control". In Neural Networks for Control Systems: Principles and Applications (K. Warwick, G. R. Irwin, and K. J. Hunt, eds.), Control Engineering Series, pp. 31-50, Peter Peregrinus, 1992.

[94] Naira Hovakimyan, Rolf Rysdyk, and Anthony Calise. "Dynamic neural networks for output feedback control". International Journal of Robust and Nonlinear Control. Vol.11, No.1, pp.23-39, 2001.

[95] K. J. Hunt, D. Sbarbaro, R. Zbikowski, and P. J. Gawthrop. "Neural networks for control systems: a survey". In Neuro-Control Systems: Theory and Applications (M. M. Gupta and D. H. Rao, eds.), IEEE Press, 1993.

[96] Naira Hovakimyan, Flavio Nardi. Anthony Calise, and Hungu Lee. "Adaptive output feedback control of a class of nonlinear systems using neural networks". International Journal of Control, Vol.74, No.12, pp.1161-1169, 2001.

[97] K. J. Hunt and D. Sbarbaro. "Neural networks for systems modeling and adaptive control". In proceedings of the IEEE Workshop on Neural Nets for Systems Applications, Prague, Czechoslovakia, 1991.

[98] Sanjay P. Bhat, and Dennis S. Bernstein. "Nontangency-based Lyapunov tests for convergence and stability in systems having a continuum of equilibria". In the SIAM Journal on Control and Optimization, Vol. 42, No. 5, pp. 1745-1775, 2003.

[99] Z. Li, C.B. Soh, and X. Xu. "Lyapunov stability of a class of hybrid dynamic systems". In Automatica, Vol. 36, No. 2, pp. 297-302, February 2000.

[100] N. P. Bhatia, and G. P. Szego. "Stability theory of dynamical systems". SpringerVerlag, Berlin, 1970.

[101] H. K. Khalil. "Nonlinear Systems", $2^{\text {nd }}$ Ed. Prentice Hall, NJ, 1996.

[102] N. N. Krasovskii. "Problems of the theory of stability of motion". Stanford University Press, Stanford, CA, 1963.

[103] J. P. LaSalle. "Some extensions of Lyapunov's second method". IRE Transactions, CT-7, pp. 520-527, 1960.

[104] R. K. Brayton, and C. H. Tong. "Constructive stability and asymptotic stability of dynamical systems". IEEE Transactions on Circuits Systems, Fundamental Theory and Applications, Vol. 27, pp. 1121-1130, 1980.

[105] H. D. Chiang, and J. S. Thorp. "Stability regions of nonlinear dynamical systems". IEEE Transactions on Automation and Control, Vol. 34, pp. 1229-1241, 1989.

[106] N. Michel, N. R. Sarabudla, and R. K. Miller. "Stability analysis of complex dynamical systems”. Circuits Systems Signal Processing 1, pp. 171-202, 1982. 
[107] Y. Ohta. "Computer generated Lyapunov functions for a class of nonlinear systems". IEEE Transactions Circuits Systems, Vol. 40, pp. 343-354, 1993.

[108] Charles E Metz. "Basic principles of ROC analysis", Seminars in Nuclear Medicine, Vol. 8, No. 4, pp. 283-298, 1978.

[109] J. A. Hanley, and B. J. McNeil "The meaning and use of the area under the receiver operating characteristic (ROC) curve", Radiology, Vol. 143, pp. 29-36, 1982.

[110] Martin Mladenovski. "Information fusion schemes for real time risk assessment in adaptive control systems", MS thesis submitted to the Lane Department of Computer Science and Electrical Engineering, West Virginia University, WV, 2004.

[111] D. E. Rumelhart and J.L. McClellan. "Parallel distributed processing: explorations in the microstructure of cognition", The MIT Press, Cambridge, MA, 1986.

[112] C. Bishop. "Neural networks for pattern recognition", Oxford University Press, Oxford, UK, pp.133-135, 326-329, 1995.

[113] M. Heywood and P. Noakes. "A framework for improved training of sigma-pi networks, IEEE Transaction on Neural Networks, Vol.6, No. 4, pp. 893-902, 1995.

[114] B. Widrow, and M. E. Hoff. "Adaptive Switching Circuits", IRE WESCON Convention Record, IRE, New York, NY, pp. 96-104, 1960.

[115] B. Widrow. "Self-Organizing Systems", Edited by M. C. Yovitz, et al, Spartan Books, Washington, DC, pp. 435-461, 1962.

[116] C. Koch, and T. Poggio. "Multiplying with synapses and neurons, Single Neuron Computation, 1992, Chap 12, pp 315-345.

[117] J. A. Feldman, D. H. Ballard. "Connectionist models and their properties". Cognitive Science, Vol. 6, pp. 205-254, 1982.

[118] C. L. Gile, G. Z. Sun, H. H. Chen, Y. C. Lee, and D. Chen. "Higher order recurrent networks \& grammatical inference" in D.S.Touretzky "Advances in Neural Information Processing Systems, pp. 380-387, Morgan Kaufmann Pubs, 1990. 


\section{Appendix A}

\section{Proof for Theorem 3.1 Stability of the DCS}

Theorem 3.1 Let $V(G, t): \mathbf{O} \subset \mathfrak{R}^{D} \rightarrow \mathfrak{R}$ be a scalar function constructed for the map $\mathrm{G}\left(\mathbf{M}, \mathbf{W}_{D \times N}, \mathbf{C}_{N \times N}\right): \mathbf{I} \subset \mathfrak{R}^{D} \rightarrow \mathbf{O} \subset \mathfrak{R}^{D}$ generated by the online learning neural network from an input manifold $\mathbf{M} \in \mathbf{I} \subset \mathfrak{R}^{D}$. If $\boldsymbol{M}$ remains fixed, then for any $\mathcal{E}>0$ we can find an integer $\delta>0$ such that for all $t>\delta$, we have $V(G, t)<\delta$.

Proof Learning in DCS is a time-varying process whose state changes according to the difference relations

$$
\frac{\Delta \mathbf{X}}{\Delta t}=\left[\begin{array}{c}
\frac{\Delta \mathbf{x}_{W}}{\Delta t} \\
\frac{\Delta \mathbf{x}_{C}}{\Delta t}
\end{array}\right]=\left[\begin{array}{l}
f_{W}\left(\mathbf{x}_{W}, \mathbf{x}_{C}\right) \\
f_{C}\left(\mathbf{x}_{C}, \mathbf{x}_{W}\right)
\end{array}\right]
$$

The first step of the stability analysis is the construction of a Lypaunov function that is non-increasing over the state trajectories of the DCS learning. The accuracy of the DCS neural network map generated from a given input manifold $\mathbf{M} \in \mathbf{I} \subset \mathfrak{R}^{D}$ is measured in order to set up a Lyapunov function for the DCS. The modeling accuracy of the DCS is measured in terms of the amount of topology of the input manifold that is being preserved by the neural network map. A function is formulated in Section 3.2 in Chapter 3 that is a raw measure of the difference in probability density functions (PDF) of the map generated by the DCS neural network and the input manifold. A Lyapunov function for the DCS is formulated in Section 3.2 in Chapter 3 and is given here.

$$
V=\frac{1}{N} \sum_{\mathbf{m} \in \mathbf{M}}\left\|\mathbf{m}-\mathbf{w}_{b m u(m)}\right\|
$$

The function, $V$ measures the distance between the data patterns of the given stationary data manifold and the corresponding best matching units $(b m u)$. The following 
conditions apply to the function, $V$ given in (9.2). As a result, the constructed function is a valid Lyapunov function.

$$
\begin{aligned}
& V(0)=0 \\
& \left\|\mathbf{m}-\mathbf{w}_{i}\right\|>0, N>0 \Rightarrow V \geq 0
\end{aligned}
$$

The objective of the proof is to show that

$$
\frac{\Delta V}{\Delta t} \leq 0
$$

Since $\Delta t>0$, it is evident from the Equation (9.4) that the numerator $\Delta V$ determines the sign in question. The objective then, is to show that $\Delta V \leq 0$.

Recall the weight update rule followed in the DCS (refer to Chapter 2).

$$
\Delta \mathbf{w}_{i}= \begin{cases}\varepsilon_{\mathrm{bmu}}\left(\mathbf{m}-\mathbf{w}_{i}\right) & \text { if } i \equiv \operatorname{bmu}(\mathbf{m}) \\ \varepsilon_{\mathrm{nbr}}\left(\mathbf{m}-\mathbf{w}_{i}\right) & \text { if } i \in\{\operatorname{nbr}(\operatorname{bmu}(\mathbf{m}))\} \\ 0 & \text { otherwise }\end{cases}
$$

In the above equation, $0<\varepsilon_{n b r}<\varepsilon_{b m u}<1$ are predefined constants known as the learning rates that define the momentum of the update process. According to Equation (9.5), there are two possible ways for the weight vectors of a neuron to be updated (changed). For a randomly drawn input data pattern, $\mathbf{m}_{1} \in \mathbf{M} \in \mathbf{I} \subset \mathfrak{R}^{D}$, the weight vectors of a DCS neural network can be updated as follows.

- As the best matching unit of input data pattern, $\mathbf{m}_{1} \in \mathbf{M} \in \mathbf{I} \subset \mathfrak{R}^{D}$, and as the best matching unit of some other input data pattern, $\mathbf{m}_{2} \in \mathbf{M} \in \mathbf{I} \subset \mathfrak{R}^{D}$.

- As a neighbor (connected node) of the best matching unit of input data pattern $\mathbf{m}_{1} \in \mathbf{M} \in \mathbf{I} \subset \mathfrak{R}^{D}$

Let us consider the first case, where the weight vectors of the best matching units are updated. In this case, the second term of Equation (9.5) may be neglected. Consider a DCS network with weight vector, $\mathbf{w}_{i}$ such that it is the best matching unit for two input data 
patterns $\mathbf{m}_{1}, \mathbf{m}_{2} \in \mathbf{M} \in \mathbf{I} \subset \mathfrak{R}^{D}$. Figure Ap.A - 1 shows a snapshot of the DCS neural network indicating the location of the weights before and after the update, $\mathrm{w}_{\text {iold }}$ and $\mathrm{w}_{\text {inew }}$ respectively. The weight vectors, $\mathbf{w}_{i \text { old }}, \mathbf{w}_{i \text { new }}$ in Figure Ap.A - 1 represent the location of the weight vectors before and after the update process respectively. The arrow between weight vectors $\mathbf{w}_{i \text { old }}, \mathbf{w}_{i \text { new }}$ shows the direction of the weight vector adaptation. The measure, $r_{1 \mathrm{~b}}$, represents the distance between weight vector, $\mathbf{w}_{i \text { old }}$, and the data pattern, $\mathbf{m}_{1}$ before the update process. The measure $r_{2 \mathrm{a}}$ represents the distance between weight vector, $\mathbf{w}_{i \text { new }}$ and the data pattern, $\mathbf{m}_{2}$ after the update process. The dotted circle around the input data patterns, $\mathbf{m}_{1}$ and $\mathbf{m}_{2}$ represent their radii of activation, i.e. if a neuron is within this radius it is considered as the BMU of that data pattern.

Let the increase in distance between the weight vector, $\mathbf{w}_{i \text { new }}$ and the data pattern, $\mathbf{m}_{1}$ due to movement of the weight vector, $\mathbf{w}_{i \text { old }}$ away from $\mathbf{m}_{1}$ be $I$. Let the decrease in distance between the weight vector, $\mathbf{w}_{i \text { new }}$ and the data pattern, $\mathbf{m}_{2}$ due to movement of the weight vector, $\mathbf{w}_{i \text { old }}$ towards $\mathbf{m}_{2}$ be $D$. Figure Ap.A - 2 shows the snapshot of DCS network overlaid with the increase and decrease factors $I$ and $D$. In order for $\Delta V \leq 0$, the increase factor, $I$ must be less than or equal to the decrease factor, $D$. 


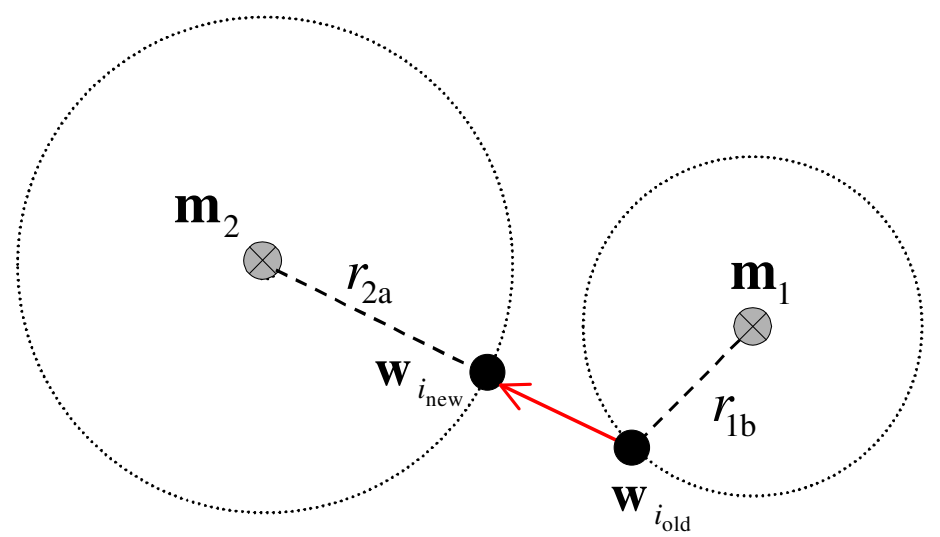

Figure Ap.A - 1 Snapshot of the DCS weight vectors before and after the update

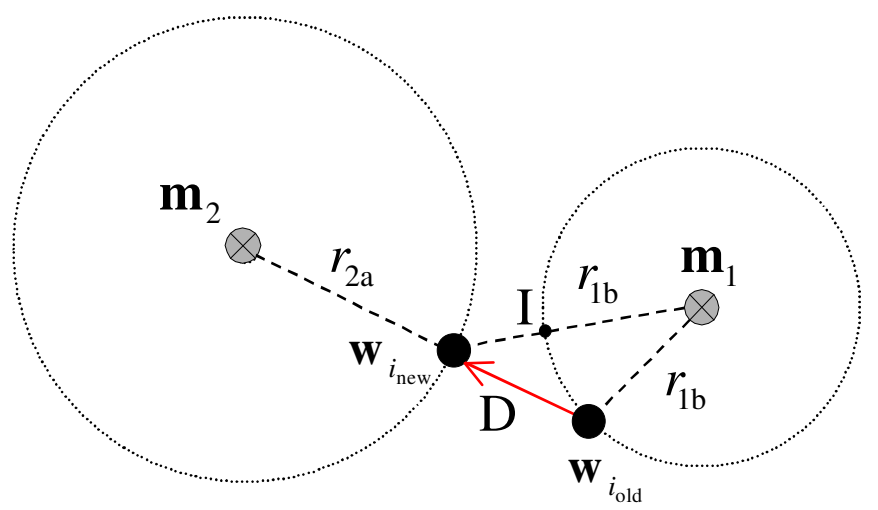

Figure Ap.A - 2 Snapshot of the DCS weight increase and weight decrease factors

As shown in Figure Ap.A - 2 there are several triangles but our interest is within the triangle formed by $\mathbf{m}_{1}, \mathbf{w}_{i \text { old }}$, and $\mathbf{w}_{i \text { new }}$ the sides of which are $r_{1 \mathrm{~b}}, D$, and $r_{1 \mathrm{~b}}+I$. Using triangular in-equality the following condition holds.

$$
\begin{aligned}
& r_{1 \text { old }}+D \geq r_{1 \text { old }}+I \\
& \Rightarrow I \leq D
\end{aligned}
$$

This condition proves that $\Delta V \leq 0$ for the case when weight vectors of best matching units are updated using the weight update rule given in Equation (9.6). 
For the second case, when the weight vector is updated as a neighbor of the best matching unit of a data pattern it may not be practical to proceed with a deterministic approach like the first case. This is because the neighbor update is highly stochastic and depends on several factors and it may not be possible to take into account all of them in our analysis. In any case, it must be realized that since $\varepsilon_{n b r}<\varepsilon_{b m u}$, the net increase in $\Delta V$ due to movement of weight vectors due to neighbor update is less significant than the net decrease in $\Delta V$ due to movement of weight vectors due to best matching unit update, i.e. $\Delta \mathbf{w}\left(\varepsilon_{n b r}\right)<\Delta \mathbf{w}\left(\varepsilon_{b m u}\right)$. Moreover it is proved by Martinetz and Schulten that as $t \rightarrow \infty, n b r(b m u(\mathbf{m})) \rightarrow \operatorname{sbu}(\mathbf{m})$ [37]. In other words, this result implies that as time progresses in DCS learning, the neighborhood function of DCS shrinks so that the second best unit (SBU) is the only neighbor of a BMU. This factor makes the net increase in $\Delta V$ due to movement of weight vectors due to neighbor update less significant than the net decrease in $\Delta V$ due to movement of weight vectors due to best matching unit update as time progresses in DCS learning, adding to the net decrease in $\Delta V$.

Based on the proven case of $\Delta V \leq 0$ for the BMU update (first case), this implies that the probability that the net change in $\Delta V$ is a decrease, and is relatively high in comparison to the probability that $\Delta V>0$. 


\section{Appendix B}

\section{Proof For Theorem 4.1: Convergence of the BMU Error}

Theorem 4.1 If a data point, $m^{*}$ is added into the data set, $M \subset \mathfrak{N}^{D}$ after $N_{0}$ learning cycles, then the DCS best matching unit error, $E_{B M U}$ will return to the previous stable error level, $E_{B M U}^{o}$ in $k$ learning cycles such that

$$
k<\frac{\log \left(\frac{E_{B M U}^{o}}{N_{o} E_{B M U}^{*}}\right)}{\log \left(1-\xi_{B M U}\right)}
$$

Proof The idea here is to provide an estimate of the number of learning cycles required by the DCS neural network learning to return the error due to BMU adjustment, $E_{B M U}$ to a previous stable level, $E_{B M U}^{o}$. Since before the addition of data point, $m^{*}$, each neuron contributed an average of $\frac{E_{B M U}^{o}}{N_{o}}$ to the BMU error, it is sufficient (though not necessary) for the error induced by the addition of $m^{*}$ to fall to the level of the average error level before perturbation, $\frac{E_{B M U}^{o}}{N_{o}}$, in order for the best matching unit error, $E_{B M U}$ to return to the value $E_{B M U}^{o}$. Computing, we have that after $k$ additional epochs, we need

$$
\begin{gathered}
\left(1-\xi_{B M U}\right)^{k} E_{B M U}^{*}<\frac{E_{B M U}^{o}}{N_{o}} \\
k \log \left(1-\xi_{B M U}\right)<\log \left(\frac{E_{B M U}^{o}}{N_{o} E^{*}{ }_{B M U}}\right) \\
k<\frac{\log \left(\frac{E_{B M U}^{o}}{N_{o} E_{B M U}^{*}}\right)}{\log \left(1-\xi_{B M U}\right)}
\end{gathered}
$$

In Equation (9.7) log is the natural log. 


\section{Proof For Theorem 4.2: Convergence of the Q-BMU Error}

Theorem 4.2 If a data point, $m^{*}$ is added into the data set, $M \subset \mathfrak{R}^{D}$ after $N_{0}$ learning cycles, then the quantized best matching unit error, $Q_{B M U}$ in DCS will return to the previous stable error level, $Q_{B M U}^{o}$ within $k$ learning cycles provided

$$
\frac{\left(1-\xi_{B M U}\right)^{k}}{k}<\frac{E_{B M U}^{o}}{N_{o} E_{B M U}^{*}}
$$

Proof Begin by noting that when $m^{*}$ is inserted, $Q_{B M U}^{o}=\frac{E_{B M U}^{o}}{N_{o}}$ and that after $k$ epochs the new quantized error will be

$$
Q_{B M U}=\frac{E_{B M U}}{N_{o}}=\frac{E_{B M U}^{o}+E_{B M U}^{*}\left(1-\xi_{B M U}\right)^{k}}{N_{o}+k}
$$

It is of interest to determine when $Q_{B M U}<Q_{B M U}^{o}$ or

$$
\begin{gathered}
\frac{E_{B M U}^{o}+E_{B M U}^{*}\left(1-\xi_{B M U}\right)^{k}}{N_{o}+k}<\frac{E_{B M U}^{o}}{N_{o}} \\
\frac{\left(1-\xi_{B M U}\right)^{k}}{k}<\frac{E_{B M U}^{o}}{N_{o} E_{B M U}^{*}}
\end{gathered}
$$

which provides the upper bound for $k$ implicitly 


\section{Appendix C}

\section{Proof for Theorem 5.1: Disconnected Highest Resource Node}

Theorem 5.1: There is a positive probability that the node with the highest resource value is disconnected from every other node of the neural network

Proof To show that there is a small, but positive probability that a randomly selected node of the SOM network is disconnected to every other node of the network is sufficient (though not necessary) to prove the theorem. This is because there is a positive probability that the randomly selected node is the node of the network with the highest resource value.

Consider a SOM of network size $N$. Let $w(i) \in W$ be the weight center of the node $i \in\{1,2, \mathrm{~K}, N\}$ of the SOM. Let $m_{i j} \in M \subset \mathfrak{R}^{D}$ be an input data pattern that has node $i$ as its best matching unit $(b m u)$. For the same data pattern, $m_{i j}$ let a node of the SOM network, $j \in\{1,2, \mathrm{~K}, N\}, j \neq i$ be the second best unit (sbu). Let $\left\{m_{j k}\right\} \in M \subset \mathfrak{R}^{D}$ represent a set of input elements (more than one input element) such that each element in the set $\left\{m_{j k}\right\}$ have node $j$ as their bmu and node $k \in\{1,2, \mathrm{~K}, N\}, k \neq j \neq i$ as their sbu.

Let nodes $i, j$ be connected to each other, $C(i, j)>0$. In other words, nodes $i$ and $j$ are neighbors, $i \in\{n b r(j)\}, j \in\{n b r(i)\}$. Let the input data pattern $m_{i j}$, the data pattern set $\left\{m_{j k}\right\}$, nodes $i$ and $j$ be oriented in space in such a manner that the following conditions hold for future adaptations.

- $\quad$ The input data pattern $m_{i j}$ has node $i$ as its bmu, and node $j$ as its sbu 
- Every input element of the data pattern set, $\left\{m_{j k}\right\}$ has node $j$ as its bmu, and node $k$ as the sbu. In other words, no element of the data pattern set $\left\{m_{j k}\right\}$ can have the node $i$ as its sbu.

- $\quad$ The node $i$ will always remain as the node of the network with the highest resource value for all future adaptations.

Note that even though the node $i$ is the bmu for only one input data pattern $m_{i j}$, the third condition is a practical scenario as the resource value is based on the distance. Let the random probability distribution, $\rho(\xi)$ be such that input data pattern $m_{i j}$ is selected before selecting any members of the set $\left\{m_{j k}\right\}$.

$$
\left\{m_{i j},\left\{m_{j k}\right\}\right\} \in \rho(\xi)
$$

Due to the competitive Hebb rule adaptation (refer to Chapter 2), the neural network adaptation due to the input data pattern $m_{i j}$ will cause the node $i$ of the network to become laterally connected to the node $j$ of the network with a connection strength of value 1 , $C(i, j)=1$, meaning that the nodes $i$ and $j$ become (fully connected) neighbors. This is due to the first condition from that states that the input data pattern $m(i)$ has node $i$ as the bmu and node $j$ as the sbu. Note that competitive Hebb rule connects bmu and sbu with a highest possible connection strength of 1 as given in Chapter 2. For the first element of $\left\{m_{j k}\right\}$ that is selected according to the random probability distribution $\rho(\xi)$, the node $j$, the $b m u$ will become laterally connected to the node $k$, the sbu with connection strength of value 1 , $C(j, k)=1$. This is due to the second condition that states that every element of the set $\left\{m_{j k}\right\}$ has the node $j$ as its $b m u$ and the node $k$ as its sbu. Note that the competitive Hebb rule not only connects the bmu and the sbu with a connection strength of 1 , but also decrements the connections between the bmu and its other (connected) neighbors (refer to 
Chapter 2). As a result, the connection strength between the nodes $i$ and $j, C(i, j)$, that was earlier set to 1 will now be decremented by multiplying it with a decrement constant, $\beta$. The process of decrementing the connection strength between the nodes $i$ and $j$ is successively repeated for every element of the set $\left\{m_{j k}\right\}$. After a certain number of repetitions, the connection strength $C(i, j)$ between the nodes $i$ and $j$ falls below a predefined threshold, $\theta$, the competitive Hebb rule disconnects the connection between the nodes $i$ and $j$ by setting the connection strength to zero, $C(i, j)=0$ (refer to Chapter 2).

Let $n$ be defined as the number of times lateral connection strength of value 1 be successively multiplied by the decrementing factor $\beta \in(0,1)$ in order for the connection strength to be equal to the threshold $\theta \in(0,1)$. In other words, if two nodes of the network that have a lateral connection strength of value 1 between them, and if the two nodes do not represent the bmu and sbu pair for an input element for $n$ consecutive trails, then the connection between the two units is terminated. The value of $n$ is computed here as

$$
n=\frac{\log (\theta)}{\log (\beta)}
$$

In this analysis, the number of elements in the set $\left\{m_{j k}\right\}$ is assumed as more than the value of $n$. This assumption is valid considering that $\mathrm{n}$ is a small finite number (usually the value of $n$ is about 4 learning cycles). In such a case, the node, $i$ with the highest resource value becomes disconnected from every other node of the network for all future adaptations.

\section{Proof for Theorem 5.2: Loss of Topology Preserving Properties}

Theorem 5.2: The process of dynamic allocation of network size and shape in SOMs that is based completely on resource values (or other local error measures) for addition of nodes into the network (usually between higher resource value neurons) does not always generate a topology preserving mapping. 
Proof In a SOM, a new neuron (if needed) is added into the network region between the neuron with the highest resource value, and its connected neighbor with the highest resource value, hrn and shn respectively. The details of this process were provided earlier in Step 1, Step 2, Step 3, and Step 4 of the neural network growing process discussed in Section 5.1 in Chapter 5.

In order to show that a neural network growing strategy that is based completely on the concept of resource values (or other forms of local error measures) does not always generate a topology preserving mapping, it is enough to show that either the mapping $\Phi_{W}$, or the inverse mapping, $\Phi_{W}^{-1}$ is not neighborhood preserving. This is because in order for the SOM, $G$ to generate a topology preserving of $M$, the mapping $\Phi_{W}$, or the inverse mapping, $\Phi_{W}^{-1}$ must be neighborhood preserving.

Consider a certain set of data set that is configured in the shape a horse-shoe as shown in Figure Ap.C - 1. Let this data serve as the input data manifold, $M \subset \mathfrak{R}^{2}$ for the SOM training. Using the above discussed dynamic allocation process, the SOM training algorithm is expected to generate a map $G \subset \mathfrak{R}^{2}$ that is topology preserving with respect to $M$.

Figure Ap.C - 2 shows the graph, $G(8)$ generated by the SOM with the presented data manifold, $M$ overlaid in the background. Note that the network size of the SOM, $G(8)$ shown in Figure Ap.C - 2 is 8 nodes, which are labeled 1 through 8 for the sake of convenience. Let us call the graph, $G(8)$ shown in Figure Ap.C - 2 as an $8^{\text {th }}$ order selforganizing neural network representation of the presented data, $M$. As shown in Figure Ap.C - 3, let the data pattern that is labeled as ' $A$ ' (top-left element of the neural net input data set) be the data pattern that is picked last during the neural network's training cycle according to a random probability distribution $\rho(\xi)$.

$$
\{\mathrm{K}, A\} \in \rho(\xi)
$$




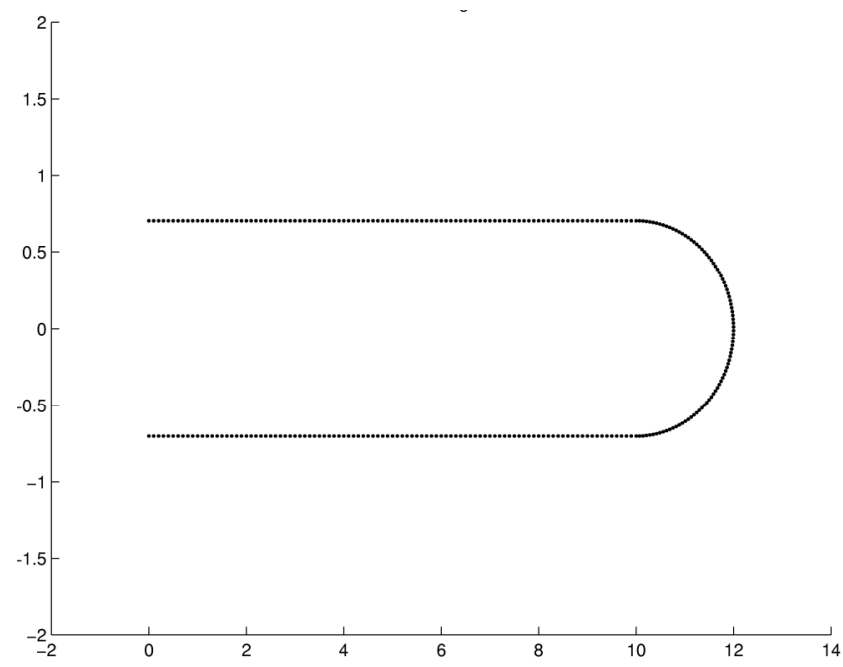

Figure Ap.C - 1 Neural network training data set

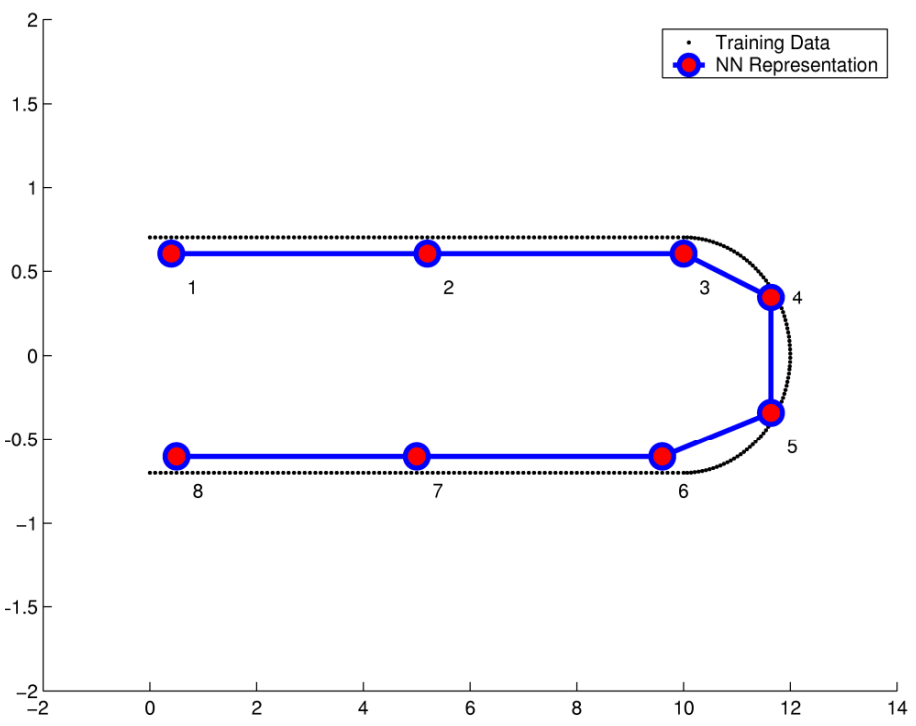

Figure Ap.C - $28^{\text {th }}$ order SOM during the inner loop of the training algorithm 
Note that a cycle here implies neural network's adaptation due to a complete sweep of all training data patterns in the input manifold. Since we are dealing with growing selforganizing maps, a cycle here is usually followed by an addition of a neuron into the network (assuming no neurons were deleted).

From Figure Ap.C - 3 it is evident that the node \#1 is the best matching unit for the input data pattern that is labeled as A. Let node \#8 be the second best unit for A. Then according to the competitive Hebb rule, nodes \#1 and \#8 become connected with connection strength of value 1 . This stage during the neural net's learning and representation is depicted in Figure Ap.C - 3. It can be seen in Figure Ap.C - 3 that the mapping, $\Phi_{W}^{-1}: G \rightarrow M$ is not neighborhood preserving because the two nodes of the SOM (nodes \#1 and \#8) that represent non-adjacent data patterns in $M$ become connected (are considered as being adjacent) in $G$. This violates the condition for map, $G$ to be a topology preserving map with respect to manifold, $M$.

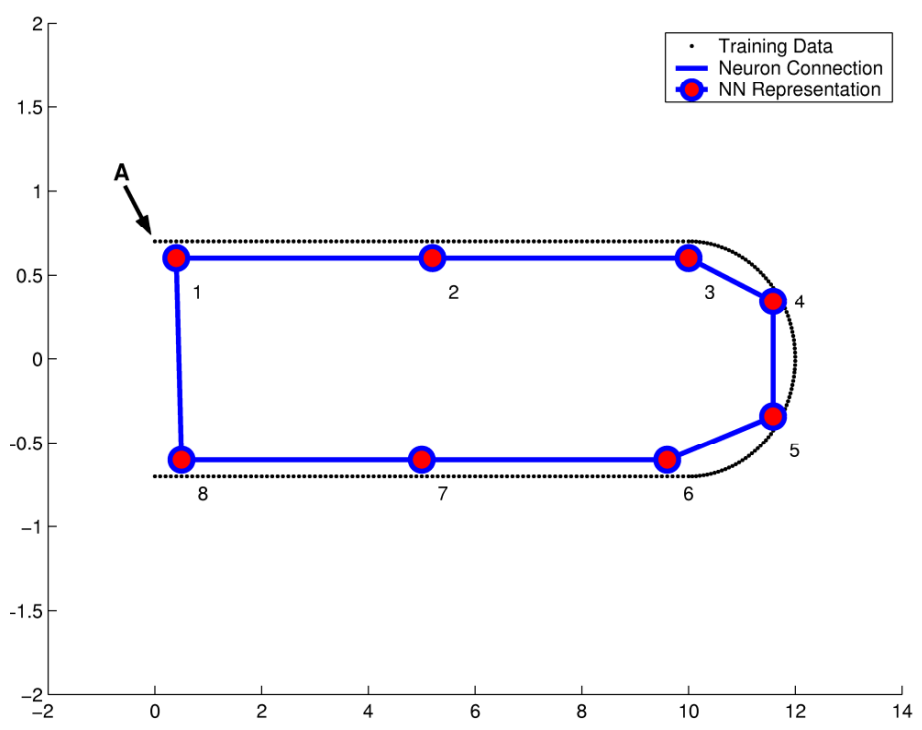

Figure Ap.C $-38^{\text {th }}$ order SOM after the end of inner loop of training algorithm

Figure Ap.C - 4 shows the representation of the SOM graph, $G$ soon after the introduction of a new node into the network. Note that since the data pattern labeled $A$ is 
selected last according to the random distribution, $\rho(\xi)$ the nodes \#1 and \#8 will remain connected (with connection strength of value 1) during the process of introducing an additional node into the network. Let node \#1 be the weight center of the neuron of the network with the highest resource value. If it is assumed that the resource value of node \#8 is higher than that of node \#2, the node \#8 becomes the second highest value node. According to the neural network growing strategy that is described earlier, a new node will be added into the network in a region between nodes \#1 and \#8.

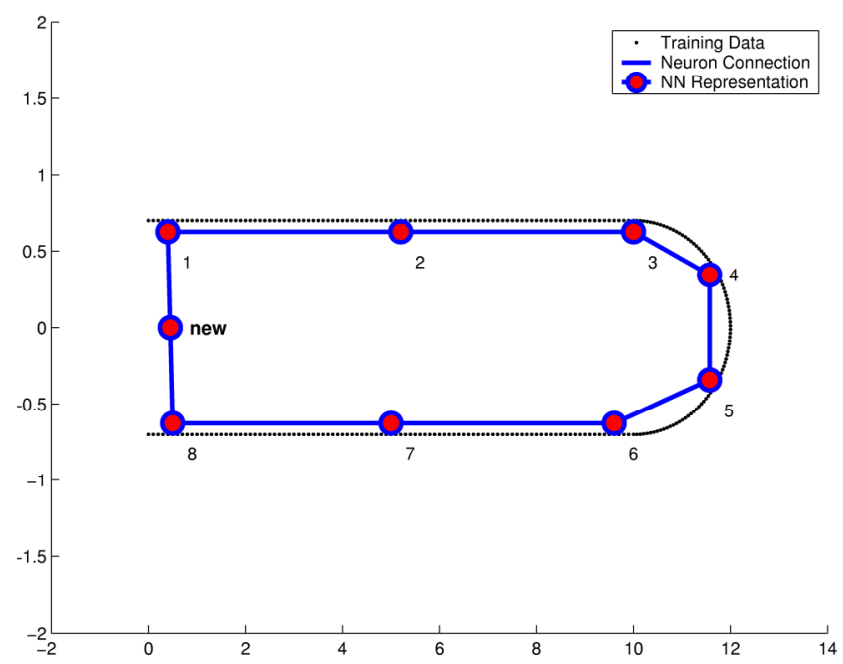

Figure Ap.C - $49^{\text {th }}$ order SOM after the addition of a new node

It is evident from Figure Ap.C - 4 that a new node is introduced into the network into a region that does not represent any data. It can be concluded that the process of dynamic allocation of network size and shape, if based completely on the resource value, cannot always generate a topology preserving mapping.

\section{Proof for Theorem 5.3: Efficient Topology Preservation}

Theorem 5.3: A neural network growing strategy that is based on addition of nodes into network regions between higher resource value nodes results in a neural network error that is higher than that generated 
by a growing strategy that is based on addition of nodes into network regions that are overlaid over input data patterns.

Proof In order to prove the theorem, it will be shown that using the neural network growing strategy followed in the original dynamic allocation algorithm does not always reduce the cumulative error of the masked Voronoi regions, and using the modified neural network growing strategy always reduces the cumulative error of the masked Voronoi regions.

In Theorem 5.2, it was shown that a neural network growing strategy that is based on adding nodes into the SOM in regions of the network between the highest and second highest resource value neurons can add a node such that it does not represent any data, i.e. no data pattern claims it as the best matching unit. In such a case, the cumulative error of the masked Voronoi region soon after the introduction of a new neuron into the network is not less than the cumulative error of the masked Voronoi region before the addition. If $V_{\text {old }}$ and $V_{\text {new }}$ represent the cumulative errors of the masked Voronoi regions before and after the neuron addition respectively, then we can write, $V_{\text {new }} \nless V_{\text {old }}$.

Let $V_{\text {old }}$ and $V_{\text {new }}$ be the cumulative errors of the masked Voronoi regions computed before and after adding the new node into the network respectively. Since the newly introduced node is overlaid over a data pattern (from Step* 2 of the modified growing algorithm), it becomes the best matching unit for at least one data pattern of the training data manifold, the data pattern over which it is overlaid, $m^{*} \in\{m(h r n)\} \in M$. Since the Euclidean distance between the newly introduced node and the data pattern over which it is overlaid is smaller (zero) than the Euclidean distance between the data pattern and its former best matching unit, the cumulative error of the masked Voronoi region, after the addition of a node, is always smaller than the cumulative error of the masked Voronoi region before the addition, $V_{\text {new }} \nless V_{\text {old }}$. 